\title{
How Dirhodium Catalyst Controls the Enantioselectivity of [3+2]-Cycloaddition between Nitrone and Vinyldiazoacetate: A Density Functional Theory Study
}

\author{
Xin Yang, ${ }^{\dagger}$ Yongsheng Yang, ${ }^{\ddagger}$ Robert Rees, ${ }^{\S}$ Qi Yang, ${ }^{\dagger}$ Zhiyue Tian, ${ }^{\ddagger}$ Ying Xue ${ }^{*^{*}}$ \\ ${ }^{\ddagger}$ College of Chemistry, Key Lab of Green Chemistry and Technology in Ministry of Education, \\ Sichuan University, Chengdu 610064, People's Republic of China. \\ ${ }^{\S}$ Data61 | CSIRO, 343 Royal Parade, Parkville Victoria 3052, Australia \\ ${ }^{\dagger}$ CSIRO Manufacturing, Bayview Avenue, Clayton 3169, Australia \\ Corresponding Author:*yxue@scu.edu.cn
}

\section{Table of Contents:}

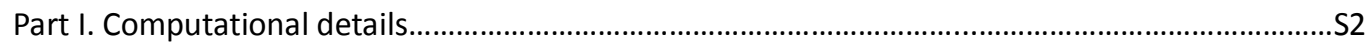

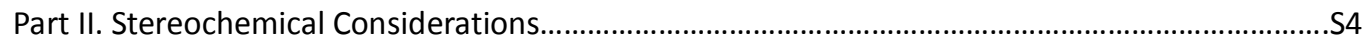

Part III. Detailed discussions on the $[3+2]$-cycloaddition reaction between vinylcarbenoid and

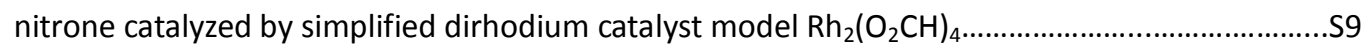
Part IV. Detailed discussions on the [3+2]-cycloaddition reaction between vinylcarbenoid and

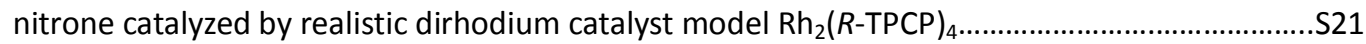
Part V. Coordinates and free energies ((SMD)M06-GD3/BS2//M06-GD3/BS1) of all stationary points .523 


\section{Part I. Computational details}

All of the DFT calculations were performed with the Gaussian 09 program package with the key word "int=ultrafine". ${ }^{1}$ The geometry optimization of all the minima and transition states involved was performed at the M06-GD3 levels of theory $^{2}$ with a basis set consisting of the 1997 Stuttgart relativistic small-core effective core-potential and basis set [Stuttgart RSC 1997 ECP] ${ }^{3}$ for rhodium, augmented with a $4 \mathrm{f}$-function $\left(\zeta_{f}(R h)=1.350\right) .{ }^{4}$ The split valence basis set $6-31 G(d, p)$ was used in the optimization and frequency calculations for all other atoms $(\mathrm{C}, \mathrm{H}, \mathrm{N}$, and $\mathrm{O})$. This composite basis set is abbreviated BS1. The vibrational frequencies were computed at the same level of theory to check whether every optimized geometrical structure is an energy minimum or a transition state. Solvent effects including radii and non-electrostatic terms were computed by the $\mathrm{SMD}^{5,6}$ model in heptane using the gas phase optimized structures at M06-GD3/BS2 level, BS2 denoting a mixed basis set of the 1997 Stuttgart relativistic small-core effective core-potential and basis set [Stuttgart RSC 1997ECP] for Rh, augmented with a $4 f$ function $(\zeta f(R h)=$ $1.350)$, and $6-311+G(2 d, 2 p)$ for all other atoms. The thermal corrections evaluated from the unscaled vibrational frequencies at the M06-GD3/BS1 level on the optimized geometries were then added to the M06-GD3/BS2 electronic energies to obtain the free energies.

The M06-GD3 functional (D3 version of Grimme's dispersion with the original D3 damping function) was used due to its superior performance for organometallic systems. ${ }^{7-9}$ Grimme's recent studies ${ }^{10,11}$ have suggested that it is important to include empirical dispersion corrections with these regularly used functionals. This is a refined version of the DFT-D method, DFT-D3, which includes an additional C8/R8 term in the dispersion correction term and takes three-body effects into consideration. This method can lead to better accuracy and transferability because the dispersion coefficients, which are calculated using an ab initio approach, and the cutoff radii are interpolated, accounting for the chemical environment of the system. 
Heavy-atom basis set definitions and corresponding pseudopotential parameters were gained from the EMSL basis set exchange library.

To obtain further insight into the electronic properties of the present system, natural bond orbital (NBO) analyses were performed at the M06-GD3/BS1 level.

\section{References:}

(1) Frisch, M.; Trucks, G.; Schlegel, H.; Scuseria, G.; Robb, M.; Cheeseman, J.; Scalmani, G.; Barone, V.; Mennucci, B.; Petersson, G.; Nakatsuji H.; Caricato M.; Li X.; Hratchian HP.; Izmaylov AF.; Bloino J.; Zheng G.; Sonnenberg JL.; Hada M; Ehara M.; Toyota K.; Fukuda R.; Hasegawa J.; Ishida M.; Nakajima T.; Honda Y.; Kitao O.; Nakai H.; Vreven T.; Montgomery JA Jr.; Peralta JE.; Ogliaro F.; Bearpark M.; Heyd JJ.; Brothers E.; Kudin KN.; Staroverov VN.; Kobayashi R.; Normand J.; Raghavachari K.; Rendell A.; Burant JC.; Iyengar SS.; Tomasi J.; Cossi M.; Rega N.; Millam JM.; Klene M.; Knox JE.; Cross JB.; Bakken V.; Adamo C.; Jaramillo J.; Gomperts R.; Stratmann RE.; Yazyev O.; Austin AJ.; Cammi R.; Pomelli C; Ochterski JW.; Martin RL.; Morokuma K.; Zakrzewski VG.; Voth GA.; Salvador P.; Dannenberg JJ.; Dapprich S.; Daniels AD.; Farkas O.; Foresman JB.; Ortiz JV.; Cioslowski J.; Fox DJ (2009) Gaussian 09, revision D.1. Gaussian Inc, Wallingford, CT.

(2) Zhao, Y.; Truhlar, D. Theor. Chem. Acc. 2008, 120, 215-241

(3) (a) Kaupp, M.; Schleyer, P. v. R.; Stoll, H.; Preuss, H. The Journal of Chemical Physics 1991, 94, 1360 (b) Bergner, A.; Dolg, M.; Küchle, W.; Stoll, H.; Preuß, H. Molecular physics 1993, 80, 1431(c) Dolg, M.; Stoll, H.; Preuss, H.; Pitzer, R. M. The Journal of Physical Chemistry 1993, 97, 5852.

(4) Hansen, J.; Autschbach, J.; Davies, H. M. L. The Journal of organic chemistry 2009, 74, 6555.

(5) Ribeiro, R. F.; Marenich, A. V.; Cramer, C. J.; Truhlar, D. G. J. Phys. Chem. B 2011, 115, 14556-14562.

(6) Marenich, A. V.; Cramer, C. J.; Truhlar, D. G. J. Phys. Chem. B 2009, 113, 6378-6396.

(7) Ganguly, G.; Malakar, T.; Paul, A. ACS Catal. 2015, 5, 2754-2769

(8) Wheeler, O. W.; Carl, D. R.; Hofstetter, T. E.; Armentrout, P. B. J. Phys. Chem. A 2015, 119, 3800-3815.

(9) Jasíkova, L.; Roithová, J. Organometallics 2013, 32, 7025-7033.

(10) Grimme, S. J. Comput. Chem. 2004, 25, 1463-1473.

(11) Steinmetz, M.; Grimme, S. ChemistryOpen 2013, 2, 115-124 


\section{Part II. Stereochemical Considerations}

The dirhodium-catalyzed [3+2]-cycloaddition reaction is highly selective for one stereoisomeric outcome. However, stereochemical issues exist that could crucially affect the reaction outcome. The two main variables in this regard are associated with: (1) the approaching side for substrate (in this case, it refers to nitrone) and (2) the vinylcarbenoid conformation and the relative orientation of nitrone.

First, let us consider the first variable (the approaching side for substrate). Asymmetric induction can take place by approach of the substrate to the Si-face of Re-face of the Rh-carbenoid. In 2011, the Davies and Musaev groups have already calculated the energies of sixteen possible $\mathrm{Rh}_{2}(R \text {-BTPCP) })_{4}$-carbene complexes (Figure S1a). ${ }^{1}$ Based on the ONIOM results, they rationalized the selectivity by a stereochemical model in which the ester group aligns perpendicular to the carbene plane, and blocks attack on its side. The substrate approaches donor/acceptor substituted rhodium-carbenoids over the donor group. When the substrate approaches over the donor group, the Re-face is blocked by the aryl ring of the ligand leaving the Si-face accessible for reactivity (Figure S2). The dirhodium catalyst $\mathrm{Rh}_{2}(R-\mathrm{TPCP}) 4$ (Figure $\mathrm{S} 1 \mathrm{~b}$ ) we investigated in this paper is structurally quite similar with $\mathrm{Rh}_{2}(R-\mathrm{BTPCP})_{4}$. Therefore, we have only taken the Si-face approach into consideration.

When we consider the other variable, the vinylcarbenoid may exist in both s-cis and s-trans conformations (Figure S3a). In our case, the vinylcarbenoid is substituted at the terminus, and reactions through the two conformers will lead to two stereochemically distinct pathways. We investigated the equilibrium (Table S1) between the s-trans and s-cis vinylcarbenoid conformers under the level of SMD-M06-GD3/BS2//M06GD3/BS1. We found there is no strongly preferred conformer among s-cis and s-trans conformations. In comparison, previously described calculations from Davies et al. ${ }^{2}$ at B3LYP/6-31G*[Rh-RSC+4f] level of theory give a slight preference for the s-trans conformer $\left(-0.2 \mathrm{kcal} \mathrm{mol}^{-1}\right)$. Besides, the barrier for rotation of $s$-trans carbenoid to s-cis carbenoid is $\Delta \mathrm{G}^{\neq}=11.6 \mathrm{kcal} \mathrm{mol}^{-1}$, 
much smaller than the free energy barriers for the $[3+2]$-cycloaddition process. Therefore, same as the conclusion from Davies, both the s-trans and s-cis carbenoid 4 should be considered into the reaction mechanism, even though the [3+2]-cycloadduct $(\boldsymbol{S})-3$ deriving from the $s$-cis conformer is not observed experimentally. With two possible orientations of nitrone, this leads to four possible approach combinations as illustrated in Figure S3b. The combinations can be considered to be endolike or exolike, in analogy to the relative location between bulky group on $\mathrm{C}^{4}$ and $-\mathrm{Ph}$ group connected to $\mathrm{C}^{3}$ on the corresponding five-membered ring $\mathbf{B}$, and are therefore classified based on this in addition to the conformation of the vinylcarbene (s-trans/endo etc.). In our paper, we will describe a detailed evaluation of reactions through all four possible combinations. To represent the mechanisms in a simpler way, we name all the possible intermediates and transition states using the prefix $\mathbf{t d}, \mathbf{t x}, \mathbf{c d}$, and $\mathbf{c x}$ to represent those involved in s-trans/endo, s-trans/exo, s-cis/endo, s-cis/exo combinations, respectively.

(a)

$\mathrm{Rh}_{2}(R-\mathrm{BTPCP})_{4}$

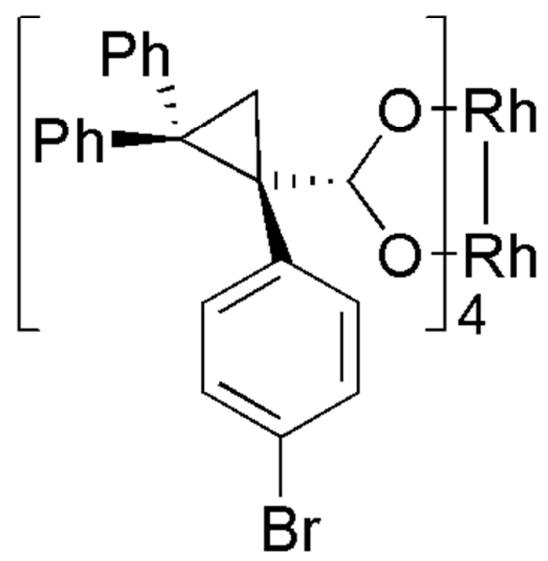

(b)

$\mathrm{Rh}_{2}(R-\mathrm{TPCP})_{4}$

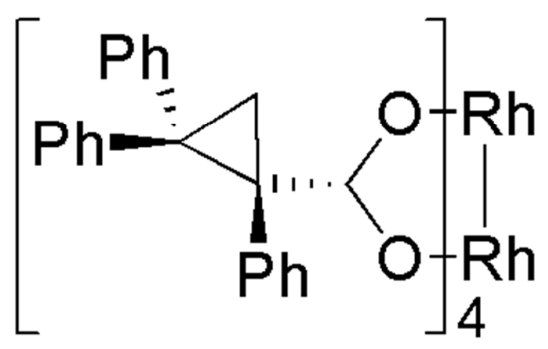

Figure S1. Chiral dirhodium catalysts. 


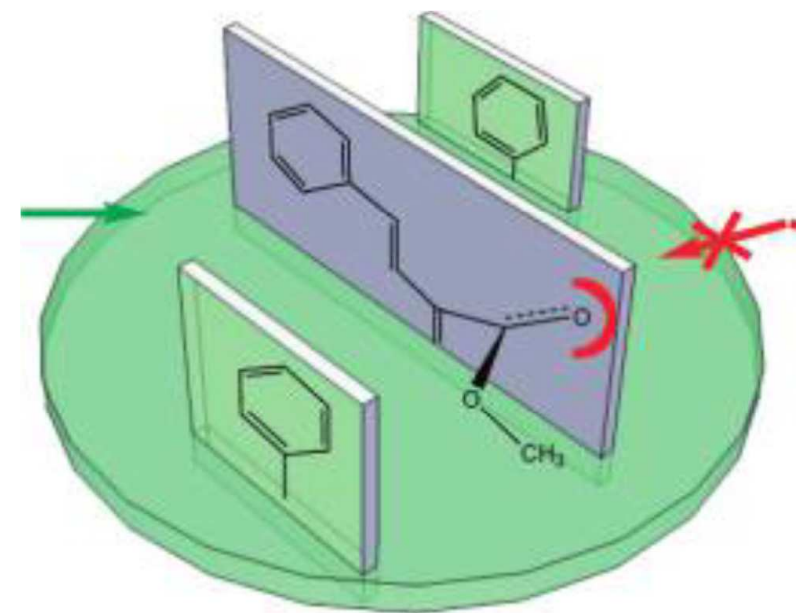

Figure S2. Davies' stereochemical model for $\mathrm{Rh}_{2}(R \text {-BTPCP })_{4}$-catalyzed transformation. Reprinted from ref 1 . Copyright 2011 American Chemical Society. 
(a)<smiles>CC=CC(=[PH2])C(C)=O</smiles>

$s-\operatorname{cis} 4$

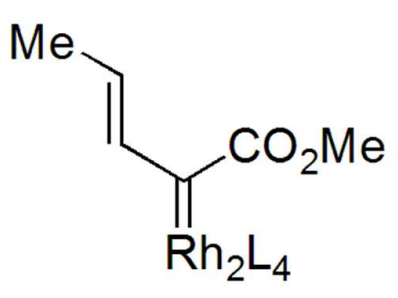

s-trans 4

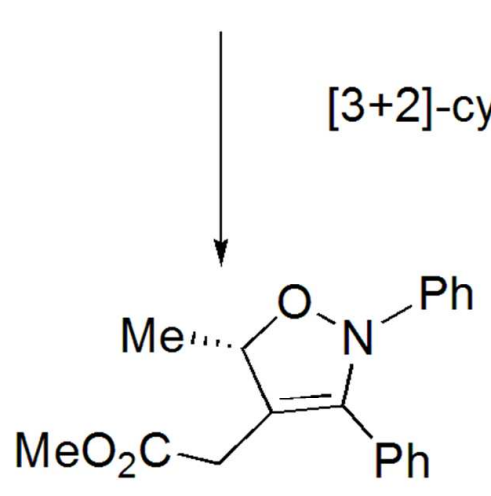

(S)-5

(b)
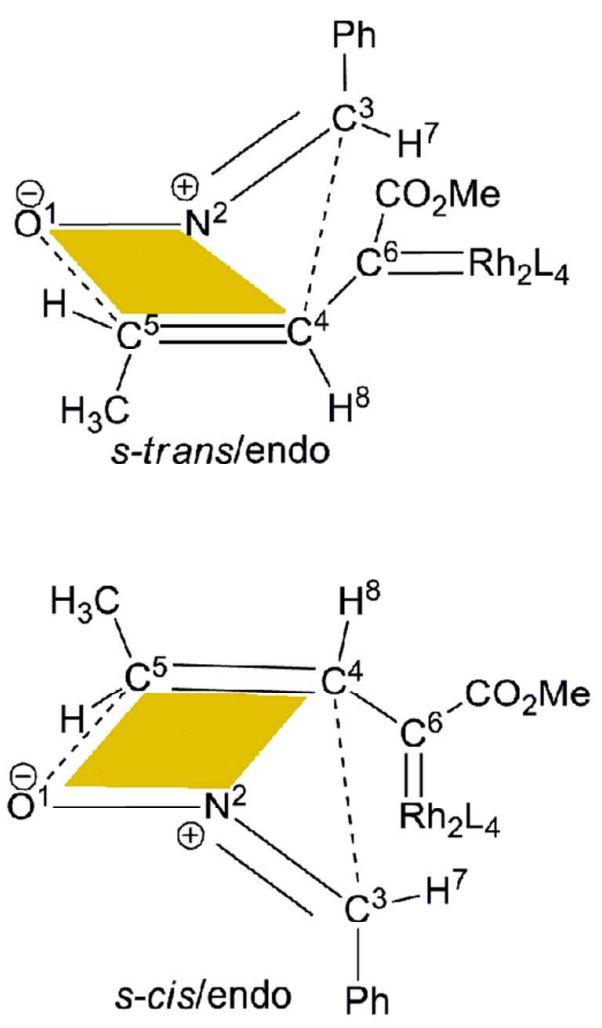
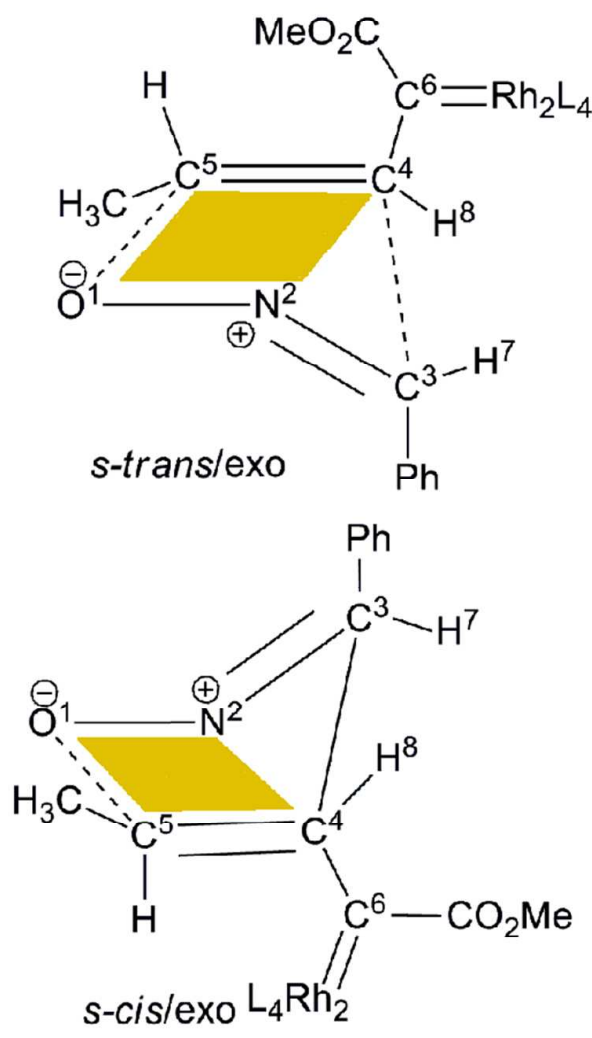

Figure S3. (a) Vinylcarbenoid conformations. (b) Combinations of nitrone approaches and vinylcarbenoid conformations. 
Table S1. Rotation between s-cis and s-trans vinylcarbenoid 4.

\begin{tabular}{|c|c|}
\hline Vinylcarbenoid $\mathbf{4}$ & $\begin{array}{c}\text { Relative free energy } \\
\left(\mathrm{kcal} \mathrm{mol}^{-1}\right)\end{array}$ \\
\hline s-trans 4 & 0 \\
\hline Transition state of rotation & 11.6 \\
\hline s-cis $\mathbf{4}$ & -0.03 \\
\hline
\end{tabular}

\section{References:}

(1) Qin, C. M. ; Boyarskikh, V; Hansen, J. H. ; Hardcastle, K. I.; Musaev, D. G. ; Davies, H. M. L. J. Am. Chem. Soc. 2011, 133, 19198-19204.

(2) Hansen, J. H.; Gregg, T. M.; Ovalles, S. R.; Lian, Y. J.; Autschbach, J.; Davies, H. M. L. J. Am. Chem. Soc. 2011, 133, 5076-5085. 
Part III. Detailed discussions on the $[3+2]$-cycloaddition reaction between vinylcarbenoid and nitrone catalyzed by simplified dirhodium catalyst model $\mathrm{Rh}_{2}\left(\mathrm{O}_{2} \mathrm{CH}\right)_{4}$.

Our mechanistic studies of the 1,3-dipolar cycloaddition reactions focus on the catalytic cycle starting from the Rh(II)-carbenoids, since previous DFT calculations have documented the detailed processes of $\mathrm{Rh}(\mathrm{II})$-carbenoid formation from vinyldiazoacetates. ${ }^{1-3}$ We employed the common procedure to simplify the bulky $\mathrm{Rh}_{2}(R \text {-TPCP })_{4}$ into $\mathrm{Rh}_{2}\left(\mathrm{O}_{2} \mathrm{CH}\right)_{4}$. This $\mathrm{Rh}_{2}\left(\mathrm{O}_{2} \mathrm{CH}\right)_{4}$ model has been widely applied to replace the realistic dirhodium catalyst, and has been able to explain the mechanism and chemoselectivity in many different systems. ${ }^{2,4-7}$
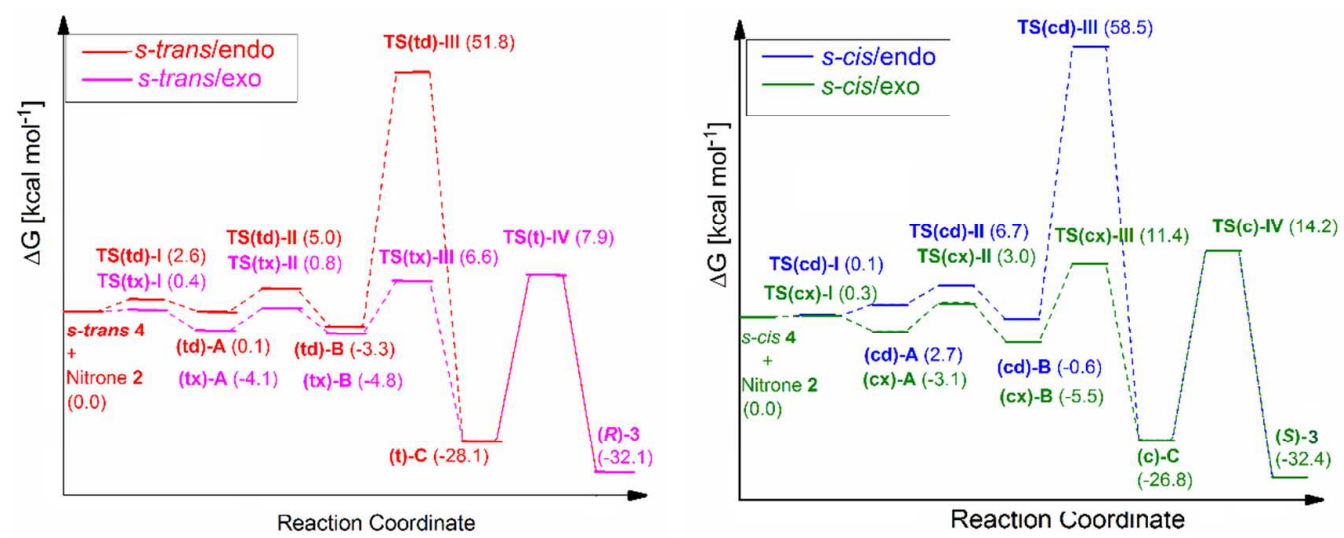

Figure S4. Gibbs free energy profile of the $\mathrm{Rh}_{2}\left(\mathrm{O}_{2} \mathrm{CH}\right)_{4}$-catalyzed [3+2]-cycloaddition of vinylcarbenoid $\mathbf{4}$ and nitrone 2 through step I followed by step II-type A mechanism. In this mechanism, the substrate (nitrone) attacks vinylcarbenoid from four different approach combinations via step I. Then, the final product $(\boldsymbol{S})-\mathbf{3}$ or $(\boldsymbol{R}) \mathbf{- 3}$ is formed through step II-type A (1,3-hydrogen migration followed by proton transfer) pathway. Energies ( $\mathrm{kcal} \mathrm{mol}^{-1}$ ) are relative to s-trans vinycarbenoid $\mathbf{4}$ and nitrone 2 and calculated using (SMD)M06-GD3/BS2//M06-GD3/BS1. 

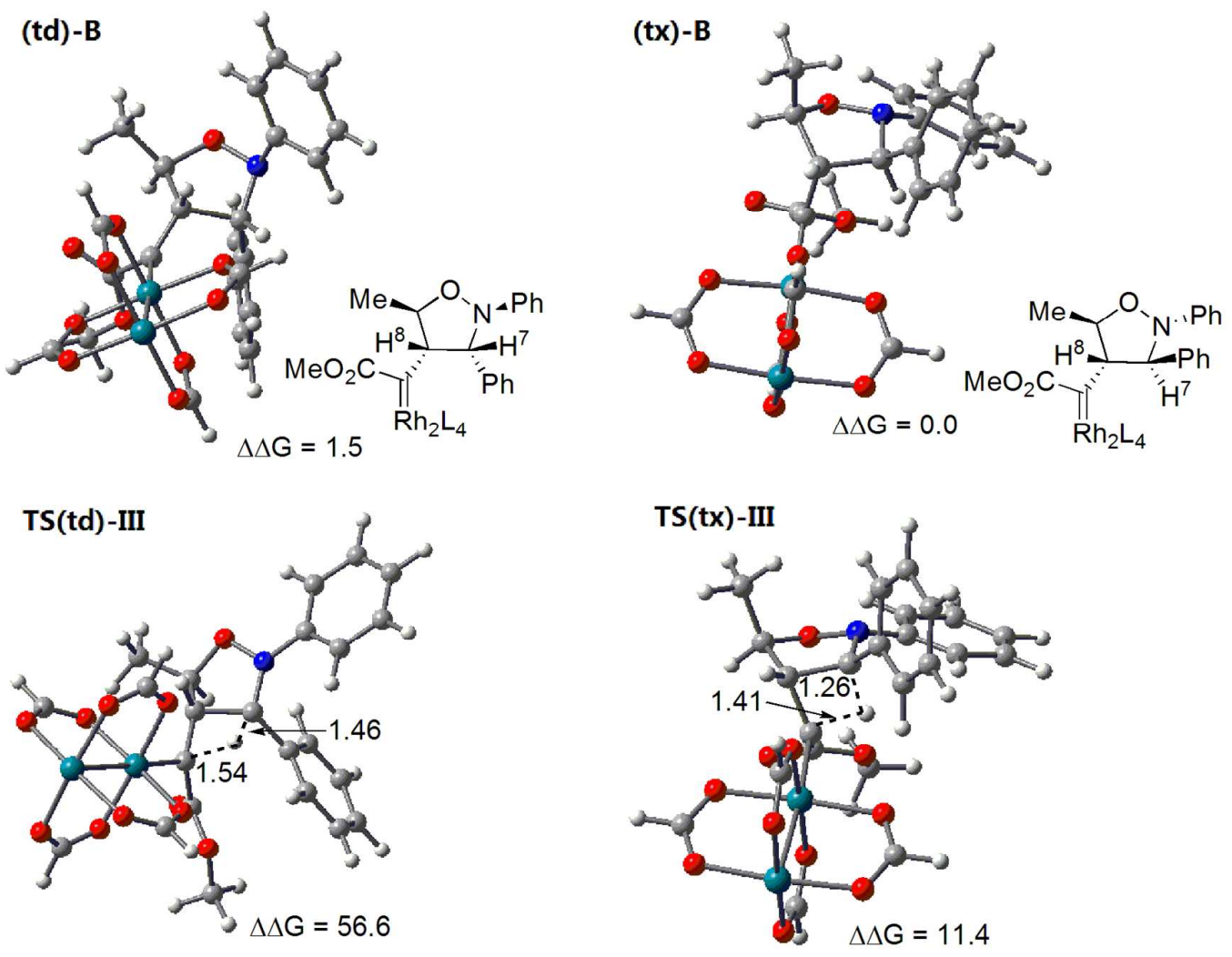

Figure S5. Optimized geometries of the five-membered ring intermediates (td)-B and (tx)-B, and the corresponding 1,3-hydrogen migration transition states TS(td)-III and TS(tx)-III in step II-type A mechanism for the $\mathrm{Rh}_{2}\left(\mathrm{O}_{2} \mathrm{CH}\right)_{4}$-catalyzed $[3+2]$-cycloaddition of vinylcarbenoid 4 and nitrone 2 . The free energies $(\Delta \Delta G)$, relative to (tx)-B, are reported in $\mathrm{kcal} \mathrm{mol}^{-1}$. All bond lengths are in $\AA$ 

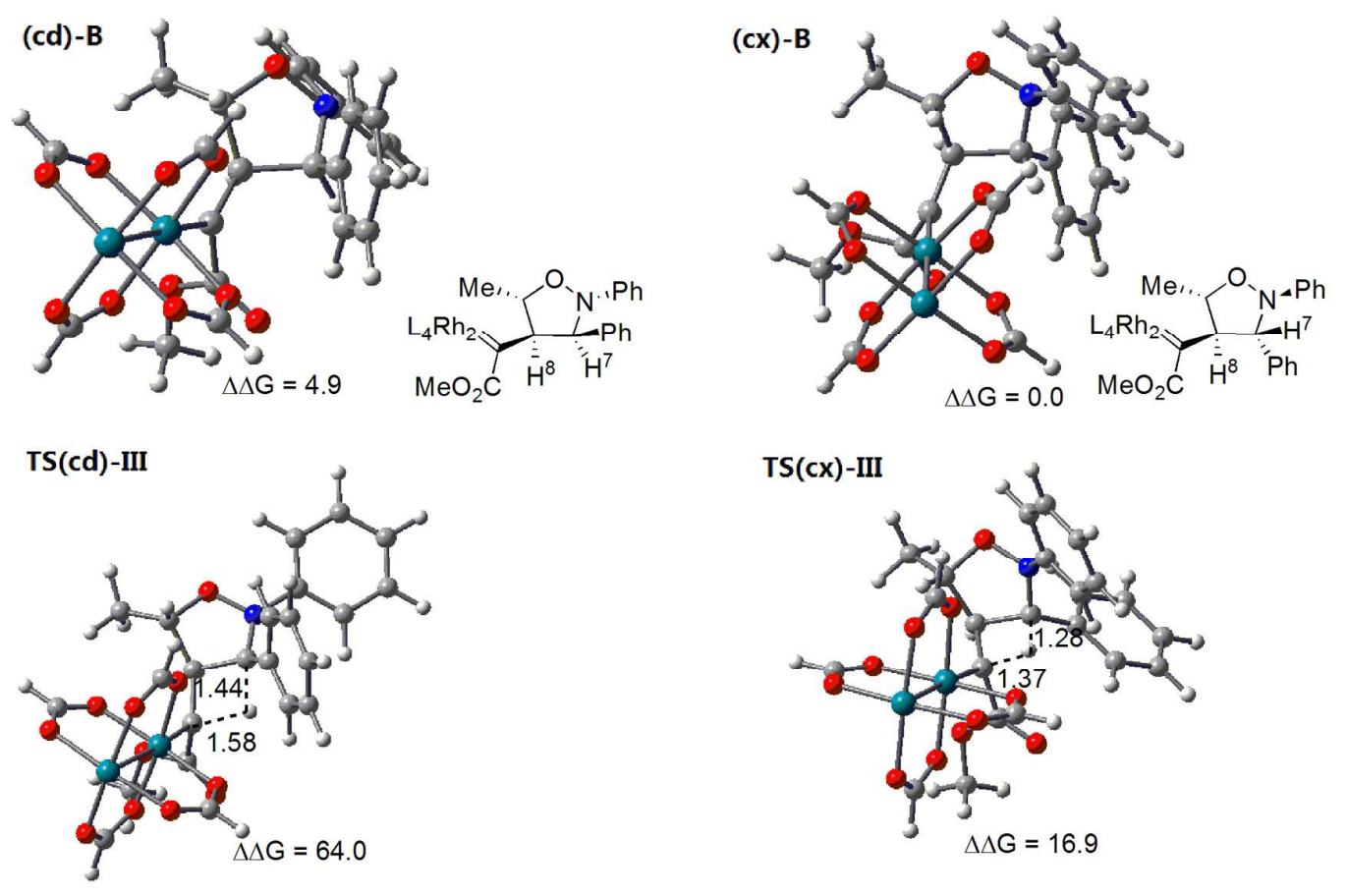

Figure S6. Optimized geometries of the five-membered ring intermediates (cd)-B and (cx)-B, and the corresponding 1,3-hydrogen migration transition states TS(cd)-III and TS(cx)-III in step II-type A mechanism for the $\mathrm{Rh}_{2}\left(\mathrm{O}_{2} \mathrm{CH}\right)_{4}$-catalyzed $[3+2]$-cycloaddition of vinylcarbenoid 4 and nitrone 2 . The free energies $(\Delta \Delta G)$, relative to (cx)-B, are reported in $\mathrm{kcal} \mathrm{mol}^{-1}$. All bond lengths are in $\AA$ 
The step II-type B includes 1,2-hydrogen migration followed by alkene isomerization (Figures S7-S11). The calculations show that starting from the five-membered ring $\mathbf{B}$, the 1,2-hydrogen migration goes through the transition state TS-V to form the intermediate $\mathbf{D}$. According to the previously proposed mechanism, the following step is an alkene isomerization that converts the alkene intermediate $\mathbf{D}$ into the final product $(\boldsymbol{R})-\mathbf{3}$ or $(\boldsymbol{S})$-3. The results indicate that the alkene isomerization includes two possible pathways: a stepwise or a concerted pathway. In the concerted alkene isomerization pathway, the $\mathrm{H}^{7}$ atom directly transfers to the carbene carbon $\mathrm{C}^{6}$ via TS-VI to form the product, whereas in stepwise alkene isomerization pathway, the $\mathrm{H}^{7}$ atom migrates to $\mathrm{C}^{4}$ (via TS-VII forming $\mathrm{E}$ intermediate) followed by $\mathrm{H}^{7}$ migration from $\mathrm{C}^{4}$ to $\mathrm{C}^{6}$ (via TS-VIII) to form the final product. Inspired by the computational studies of dirhodium-catalyzed $\mathrm{O}-\mathrm{H}$ insertion ${ }^{10}$ in which $\mathrm{Rh}_{2} \mathrm{~L}_{4}$ could work as three different reactive precursors, we also located the concerted alkene isomerization transition states $\mathrm{Rh}_{2} \mathrm{~L}_{4}$-associated TS-VI-(c) (having carbene carbon $\mathrm{C}^{6}$ as the coordinating atom) and TS-VI-(o) (having oxygen in ester group as the coordinative atom) and free transition state TS-VI-(f); this is the same as in the stepwise alkene isomerization pathways. The overall energy barriers in stepwise pathways are all substantially lower than those in the corresponding concerted pathways. However, the most favourable pathway in step II-type B among the four combinations still has high free energy barrier of $37.9 \mathrm{kcal} \mathrm{mol}^{-1}$ (from (td)-B via (td)-E-(c) to the product $(\boldsymbol{R})-\mathbf{3})$. Therefore, this step II-type B pathway is also kinetically unfeasible, in view of the fact that the dirhodium-catalyzed $[3+2]$-cycloaddition can smoothly occur at room temperature. 


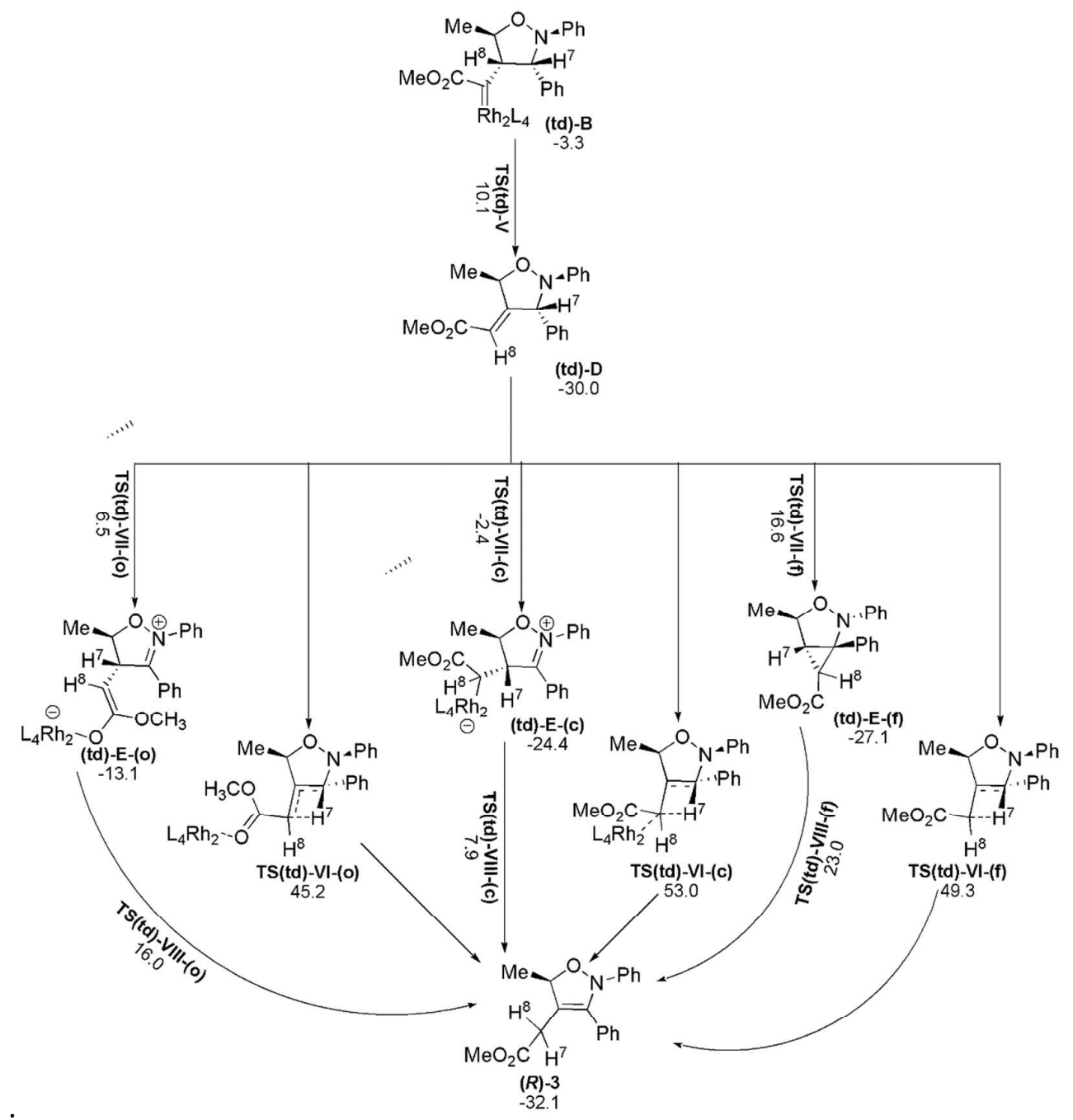

Figure S7. Free energies of intermediates in step II-type B mechanism involving intermediate (td)-B for the $\mathrm{Rh}_{2}\left(\mathrm{O}_{2} \mathrm{CH}\right)_{4}$-catalyzed [3+2]-cycloaddition of vinylcarbenoid 4 and nitrone 2. Energies $\left(\mathrm{kcal} \mathrm{mol}^{-1}\right.$ ) are relative to $s$-trans vinycarbenoid $\mathbf{4}$ and nitrone $\mathbf{2}$ and calculated using (SMD)M06-GD3/BS2//M06-GD3/BS1. 


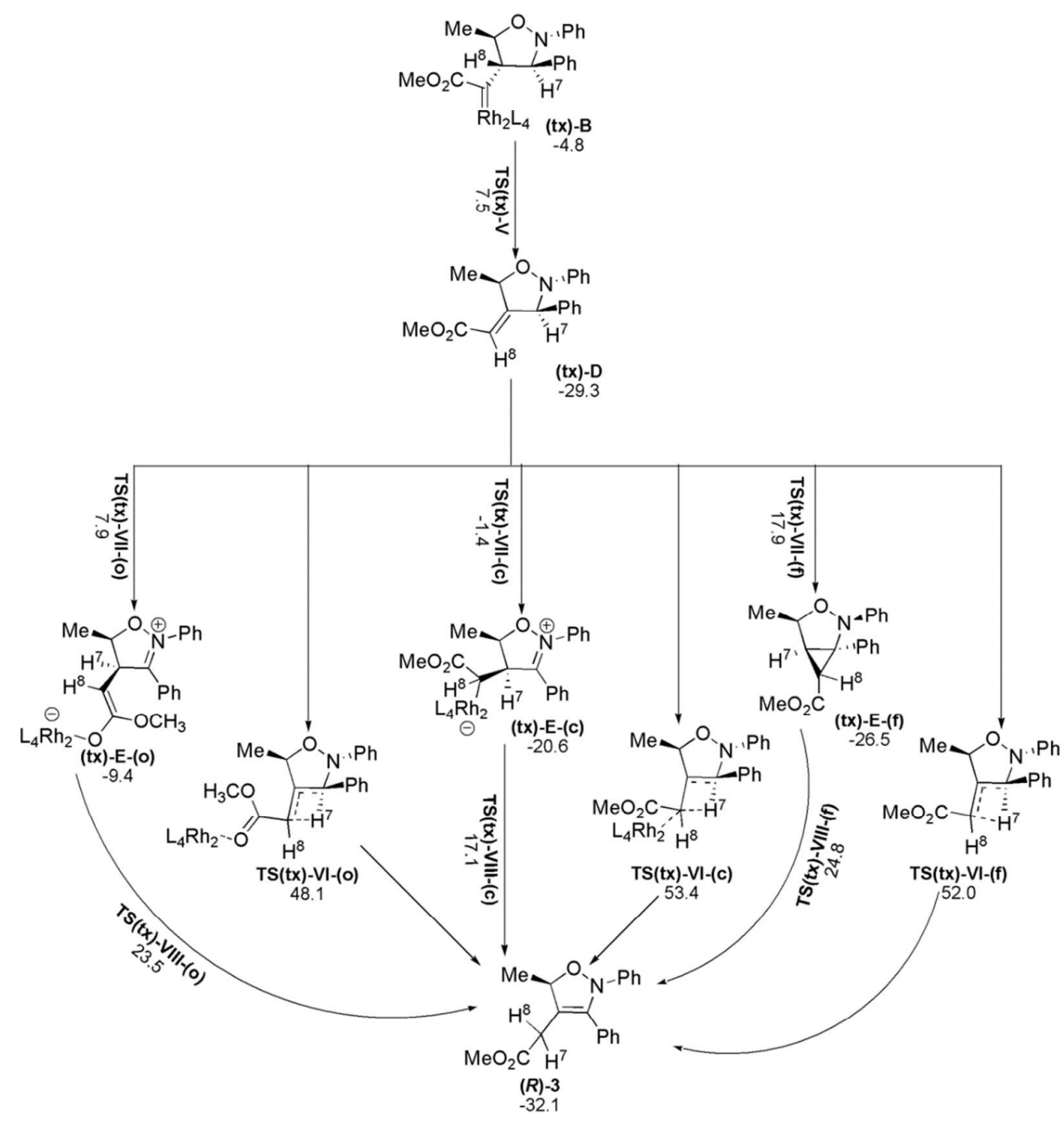

Figure S8. Free energies of intermediates in step II-type B mechanism involving intermediate (tx)-B for the $\mathrm{Rh}_{2}\left(\mathrm{O}_{2} \mathrm{CH}\right)_{4}$-catalyzed [3+2]-cycloaddition of vinylcarbenoid 4 and nitrone 2. Energies $\left(\mathrm{kcal} \mathrm{mol}^{-1}\right.$ ) are relative to $s$-trans vinycarbenoid $\mathbf{4}$ and nitrone $\mathbf{2}$ and calculated using (SMD)M06-GD3/BS2//M06-GD3/BS1. 


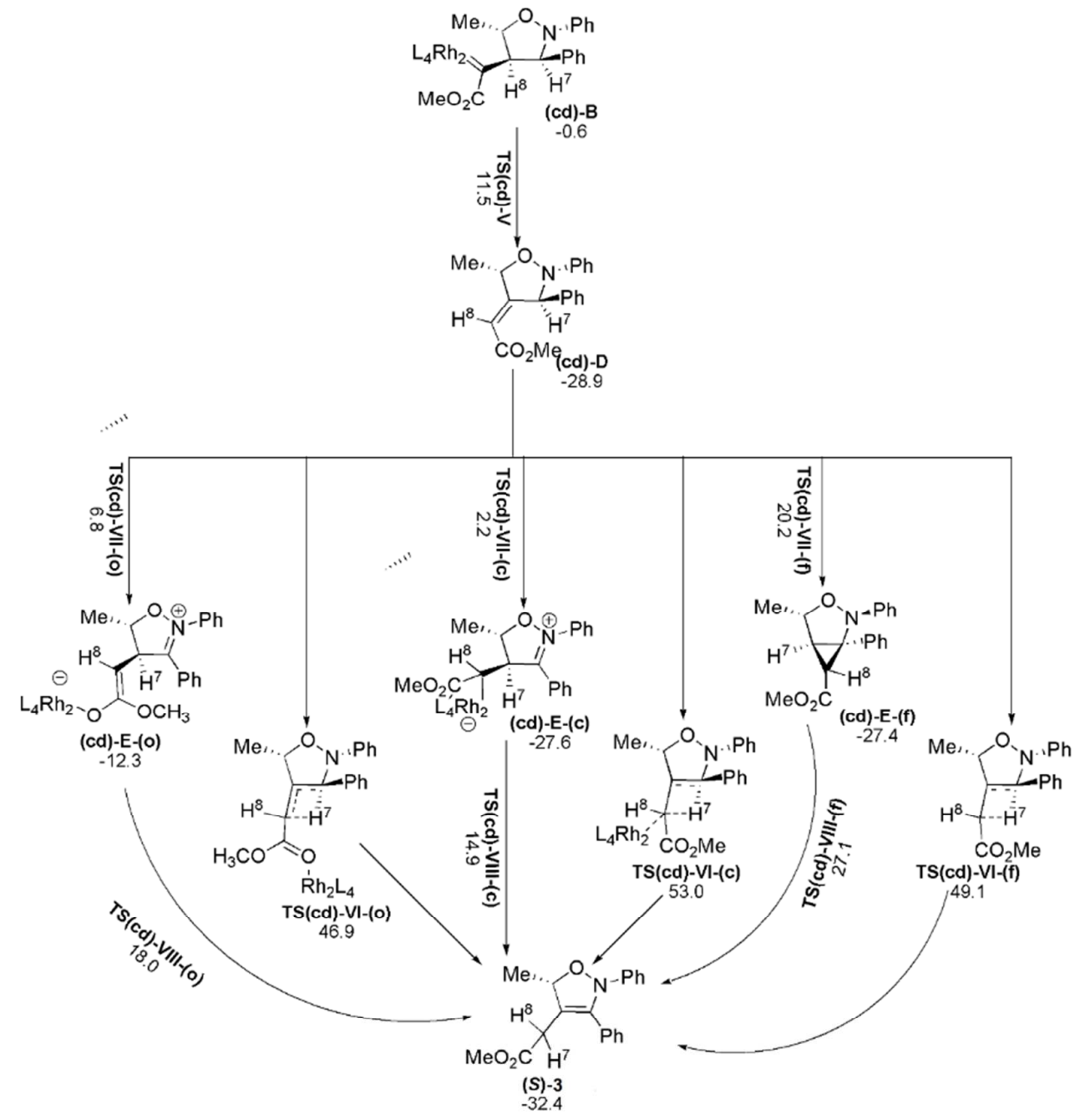

Figure S9. Free energies of intermediates in step II-type $\mathbf{B}$ mechanism involving intermediate (cd)-B for the $\mathrm{Rh}_{2}\left(\mathrm{O}_{2} \mathrm{CH}\right)_{4}$-catalyzed [3+2]-cycloaddition of vinylcarbenoid 4 and nitrone 2. Energies $\left(\mathrm{kcal} \mathrm{mol}^{-1}\right)$ are relative to s-trans vinycarbenoid 4 and nitrone $\mathbf{2}$ and calculated using (SMD)M06-GD3/BS2//M06-GD3/BS1. 


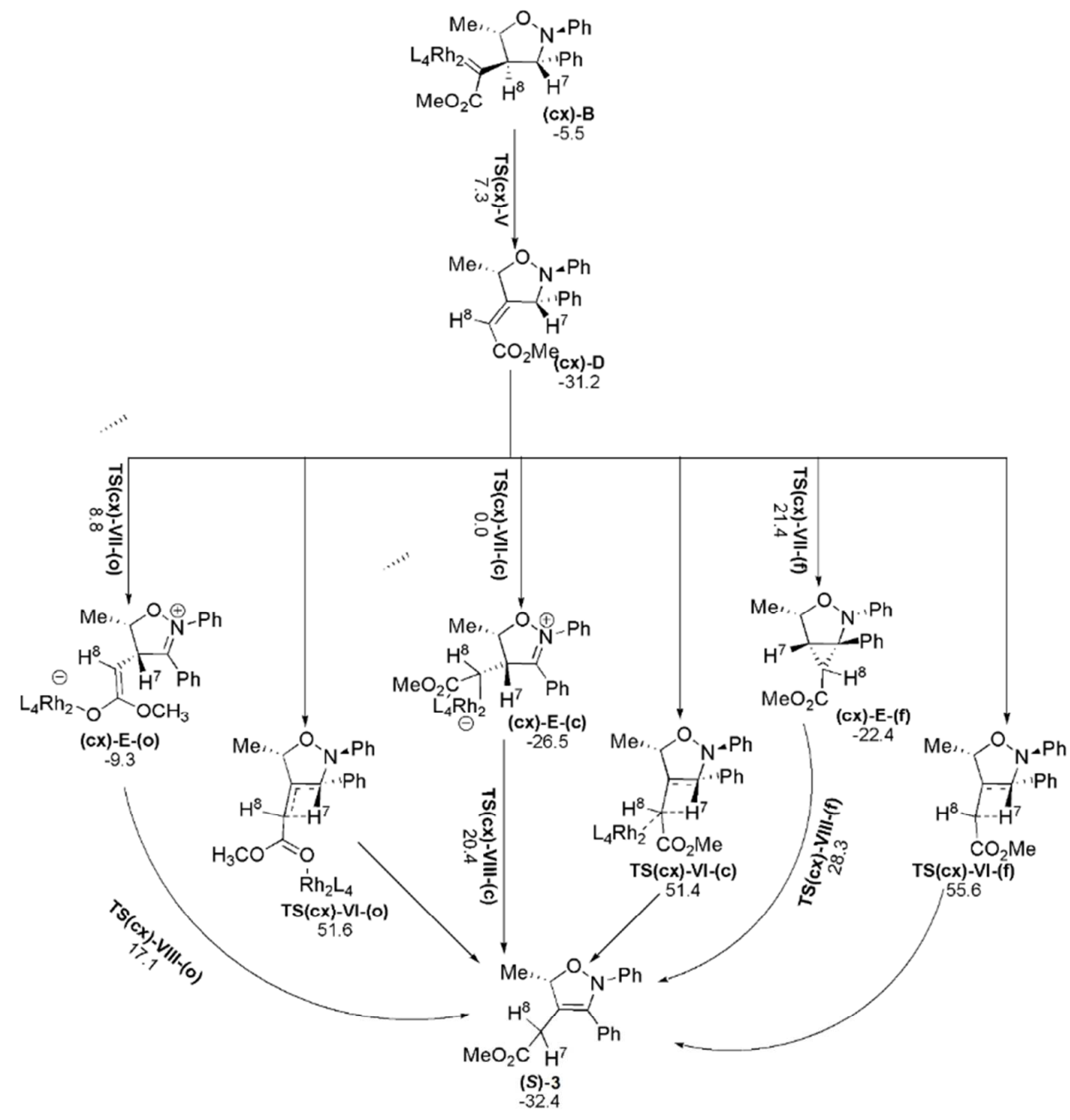

Figure S10. Free energies of intermediates in step II-type B mechanism involving intermediate (cx)-B for the $\mathrm{Rh}_{2}\left(\mathrm{O}_{2} \mathrm{CH}\right)_{4}$-catalyzed [3+2]-cycloaddition of vinylcarbenoid 4 and nitrone 2. Energies $\left(\mathrm{kcal} \mathrm{mol}^{-1}\right.$ ) are relative to $s$-trans vinycarbenoid $\mathbf{4}$ and nitrone $\mathbf{2}$ and calculated using (SMD)M06-GD3/BS2//M06-GD3/BS1. 

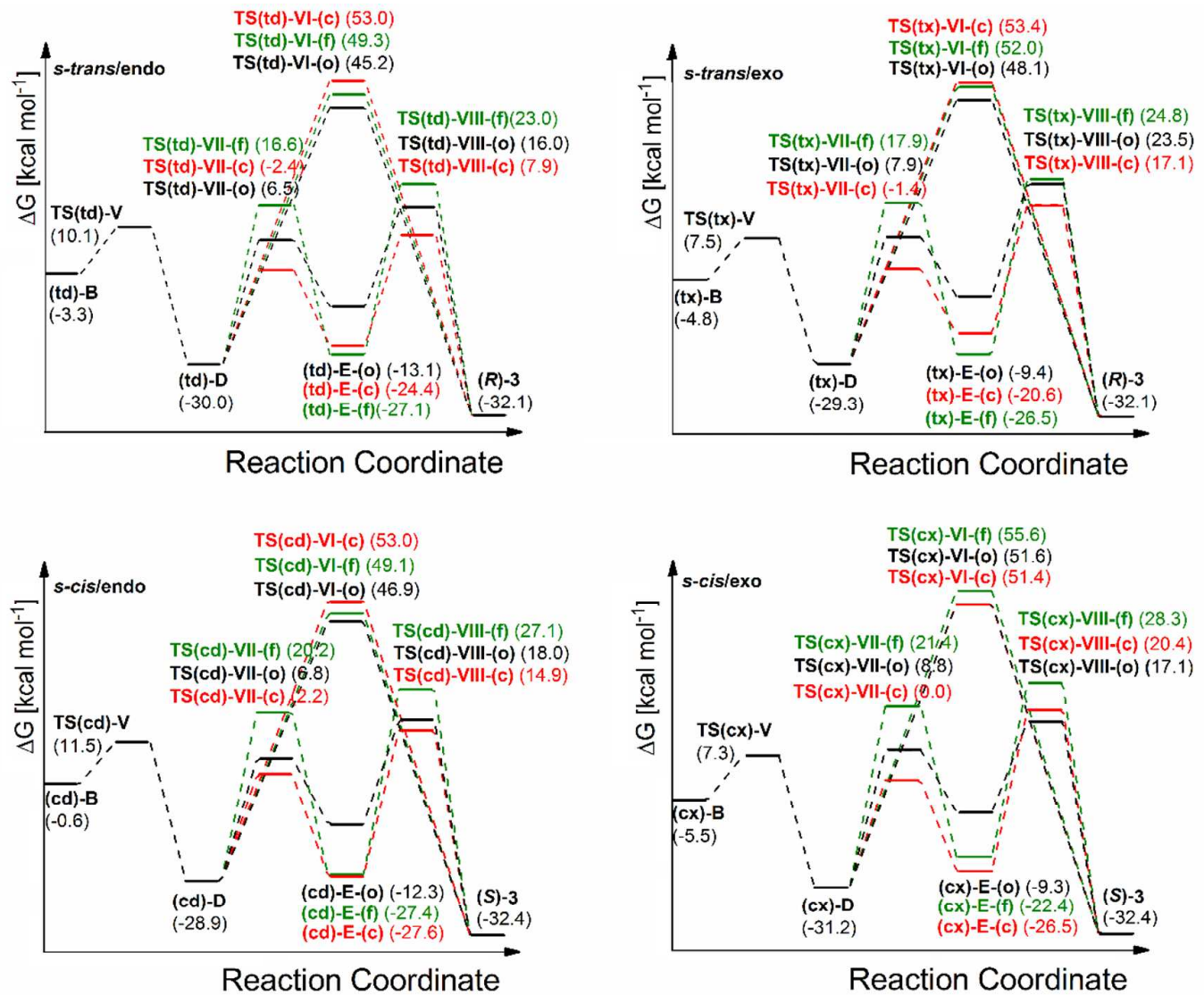

Figure S11. Gibbs free energy profile of the $\mathrm{Rh}_{2}\left(\mathrm{O}_{2} \mathrm{CH}\right)_{4}$-catalyzed [3+2]-cycloaddition of vinylcarbenoid $\mathbf{4}$ and nitrone 2 through step I followed by step II-type B mechanism. In this mechanism, the five-membered ring intermediates (td)-B, (tx)-B, (cd)-B and (cx)-B formed from step I go through step II-type B (1,2-hydrogen migration followed by alkene isomerization) pathway to generate the final product $(\boldsymbol{S})$-3 or $(\boldsymbol{R})$-3. Energies $\left(\mathrm{kcal} \mathrm{mol}^{-1}\right)$ are relative to $s$-trans vinycarbenoid $\mathbf{4}$ and nitrone 2 and calculated using (SMD)M06-GD3/BS2//M06-GD3/BS1. 
Based on the free energy profiles (Figure S4 and Figure S11), it is obvious that in the $\mathrm{Rh}_{2}\left(\mathrm{O}_{2} \mathrm{CH}\right)_{4}$-catalyzed [3+2]-cycloaddition reaction between vinylcarbenoid and nitrones, the annulation via step $\mathbf{I}$ is energetically favored in all four possible approach combinations (s-trans/endo, s-trans/exo, s-cis/endo and s-cis/exo), but step II suffers very high free energy barrier no matter the catalyst dissociation proceeds either through step II-type $\mathbf{A}$ (1,3-hydrogen migration + protonation) or step II-type B (1,2-hydrogen migration + alkene isomerization). Very surprisingly, An unexpected energy barrier decline for protonation was observed when additional $\mathrm{Rh}_{2}\left(\mathrm{O}_{2} \mathrm{CH}\right)_{4}$ was associated with $\mathrm{O}^{1}$ atom on five-membered ring intermediate $\mathbf{B}$. The computed activation free energies for protonation via transition state $\mathbf{T S}(\mathbf{t})-\mathbf{I V}-\mathbf{2} \mathbf{R} \mathbf{h}_{\mathbf{2}} \mathbf{L}_{\mathbf{4}}$ and $\mathbf{T S}(\mathbf{c})-\mathbf{I V}-\mathbf{2} \mathbf{R h}_{\mathbf{2}} \mathrm{L}_{\mathbf{4}}$ are 9.6 and $13.3 \mathrm{kcal} \mathrm{mol}^{-1}$ lower than those via $\mathbf{T S}(\mathbf{t})-\mathbf{I V}$ and TS(c)-IV, respectively (Figure 5 and Figure S12).

In order to understand order interactions among the filled and virtual orbitals, the second order Fock matrix ${ }^{8}$ was calculated to evaluate the donor-acceptor interactions using $\mathrm{NBO}^{9}$ program as implemented in the Gaussian 09 package at the M06-GD3/BS1 level. The interactions result in a loss of occupancy from the localized NBO of the idealized Lewis structure into an empty non-Lewis orbital. For each donor (i) and acceptor $(j)$, the stabilization energy $E(2)$ associated with the delocalization $i \rightarrow j$ is estimated as

$$
E(2)=\Delta E_{i j}=q_{i} \frac{F(i, j)^{2}}{\varepsilon_{j}-\varepsilon_{i}}
$$

where $q_{i}$ is the donor orbital occupancy, $\varepsilon_{j}$ and $\varepsilon_{i}$ are diagonal elements and $F(i, j)$ is the off diagonal NBO Fock matrix element. The larger the $E(2)$ value, the more intensive is the interaction between electron donors and electron acceptors, i.e. the more donating tendency from electron donors to electron acceptors and the greater the extent of conjugation of the whole system. 


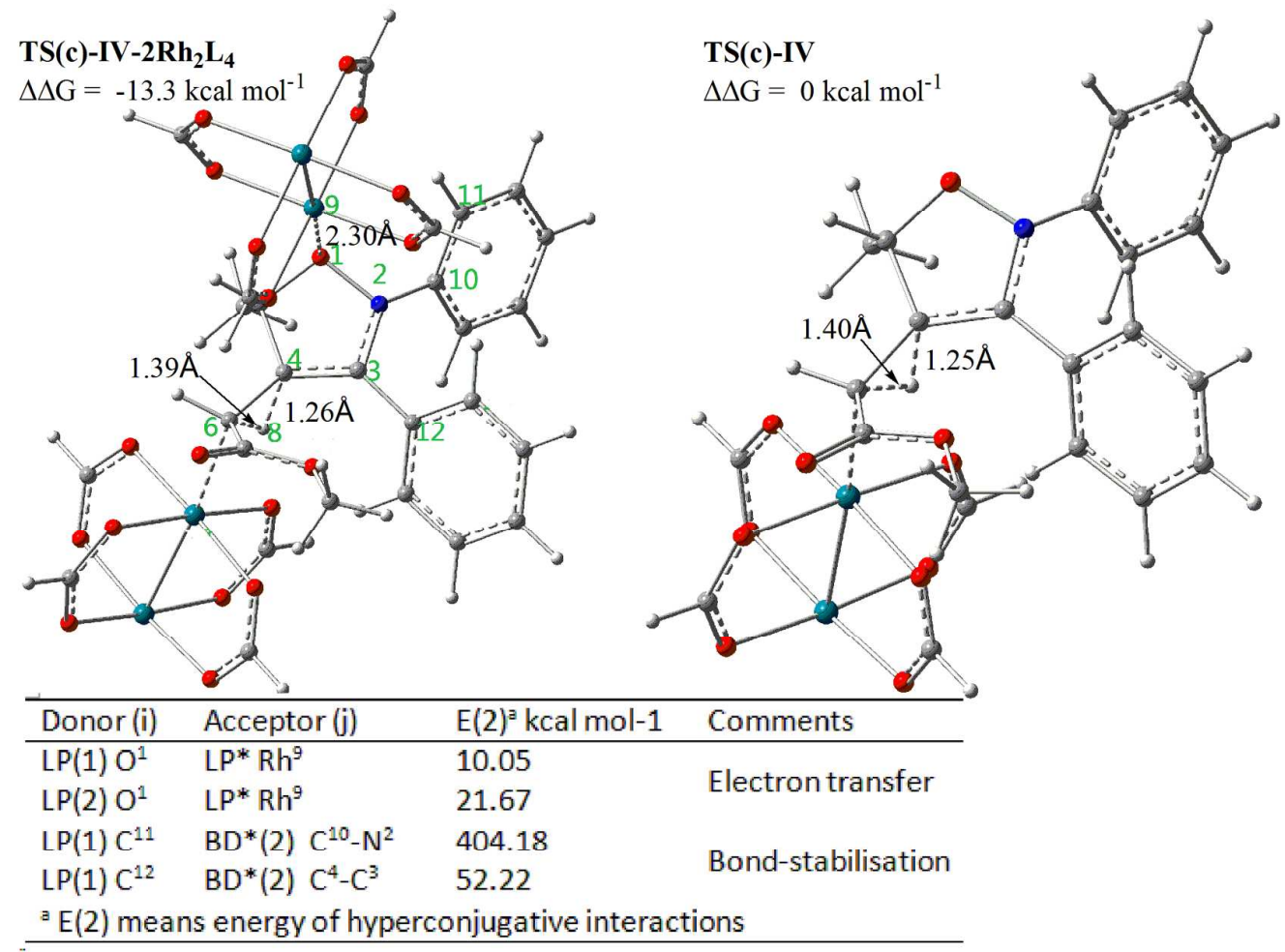

Figure S12. Optimized transition structures $\mathbf{T S}(\mathbf{c})-\mathbf{I V}-\mathbf{2} \mathbf{R} \mathbf{h}_{\mathbf{2}} \mathbf{L}_{\mathbf{4}}$ and $\mathbf{T S}(\mathbf{c})-\mathbf{I V}$ and the Second order perturbation theory analysis of Fock Matrix in NBO basis for TS(c)-IV-2Rh $\mathbf{L}_{\mathbf{4}}$. Energies are calculated using (SMD)M06-GD3/BS2//M06-GD3/BS1.

References:

1. Nakamura, E.; Yoshikai, N.; Yamanaka, M. J. Am. Chem. Soc 2002, 124, 7181-7192.

2. Nowlan, D. T.; Gregg, T. M.; Davies, H. M. L.; Singleton, D. A. J. Am. Chem. Soc 2003, 125, 15902-15911.

3. Hansen, J.; Autschbach, J.; Davies, H. M. L. J. Org. Chem. 2009, 74, 6555-6563.

4. Sizova, O. V.; Sokolov, A. Y.; Skripnikov, L. V.; Baranovski, V. I. Polyhedron 2007, 26, 4680-4690.

5. Cotton, F. A.; Feng, X. J. J. Am. Chem. Soc 1998, 120, 3387-3397.

6. Xie, Z. Z.; Liao, W. J.; Cao, J.; Guo, L. P.; Verpoort, F.; Fang, W. H. Organometallics 2014, 33, 2448-2456.

6. Boultadakis-Arapinis, M.; Gandon, V.; Prost, E.; Micouin, L.; Lecourt, T. Adv. Synth. Catal. 2014, 356, $2493-2505$.

7. Hansen, J. H.; Gregg, T. M.; Ovalles, S. R.; Lian, Y. J.; Autschbach, J.; Davies, H. M. L. J. Am. Chem. Soc. 2011, 133, 5076-5085.

8. Reed, A. E.; Curtiss, L. A.; Weinhold, F. Chem. Rev. 1988, 88, 899. 
9. Glendening, E. D.; Reed, A. E.; Carpenter, J. E.; Weinhold,F. NBO Version 3.1, TCl, University of Wisconsin, Madison, 1998.

10. Liang, Y.; Zhou, H.; Yu, Z.-X. J. Am. Chem. Soc. 2009, 131, 17783-17785; 
Part IV. Detailed discussions on the $[3+2]$-cycloaddition reaction between vinylcarbenoid and nitrone catalyzed by realistic dirhodium catalyst model $\mathrm{Rh}_{2}(R-\mathrm{TPCP})_{4}$.

The initial geometries for $\mathrm{Rh}_{2}(R-\mathrm{TPCP})_{4}$ models were created starting with the $\mathrm{X}$-ray structure of $\mathrm{Rh}_{2}(R-\mathrm{BTPCP})_{4} \cdot{ }^{1}$ All of the DFT calculations were performed with the Gaussian 09 program package with the key word "int=ultrafine". The geometry optimization of all the minima and transition states involved was performed at the M06-GD3 levels of theor ${ }^{2}$ with a basis set consisting of the 1997 Stuttgart relativistic small-core effective core-potential and basis set [Stuttgart RSC 1997 ECP] ${ }^{3}$ for rhodium, augmented with a $4 \mathrm{f}$-function $\left(\zeta_{\mathrm{f}}(\mathrm{Rh})=1.350\right) .{ }^{4}$ The split valence basis set 6-31G(d,p) was used in the optimization and frequency calculations for all other atoms ( $\mathrm{C}, \mathrm{H}, \mathrm{N}$, and $\mathrm{O})$. This composite basis set is abbreviated BS1. The vibrational frequencies were computed at the same level of theory to check whether every optimized geometrical structure is an energy minimum or a transition state. Solvent effects including radii and non-electrostatic terms were computed by the SMD ${ }^{5,6}$ model in heptane using the gas phase optimized structures at M06-GD3/BS2 level, BS2 denoting a mixed basis set of the 1997 Stuttgart relativistic small-core effective core-potential and basis set [Stuttgart RSC 1997ECP] for Rh, augmented with a $4 \mathrm{f}$ function $(\zeta f(R h)=1.350)$, and $6-311+G(2 d, 2 p)$ for all other atoms. The thermal corrections evaluated from the unscaled vibrational frequencies at the M06-GD3/BS1 level on the optimized geometries were then added to the M06-GD3/BS2 electronic energies to obtain the free energies.

References:

(1) Qin, C. M. ; Boyarskikh, V; Hansen, J. H. ; Hardcastle, K. I.; Musaev, D. G. ; Davies, H. M. L. J. Am. Chem. Soc. 2011, 133, 19198-19204

(2) Zhao, Y.; Truhlar, D. Theor. Chem. Acc. 2008, 120, 215-241

(3) (a) Kaupp, M.; Schleyer, P. v. R.; Stoll, H.; Preuss, H. J. Chem. Phys. 1991, 94, 1360.

(b) Bergner, A.; Dolg, M.; Küchle, W.; Stoll, H.; Preuß, H. Molecular physics 1993, 80, 1431. (c) Dolg, M.; Stoll, H.; Preuss, H.; Pitzer, R. M. J. Phys. Chem. 1993, 97, 5852.

(4) Hansen, J.; Autschbach, J.; Davies, H. M. L. The Journal of organic chemistry 2009, $74,6555$.

(5) Ribeiro, R. F.; Marenich, A. V.; Cramer, C. J.; Truhlar, D. G. J. Phys. Chem. B 2011, 115, 14556-14562.

(6) Marenich, A. V.; Cramer, C. J.; Truhlar, D. G. J. Phys. Chem. B 2009, 113, 6378-6396. 


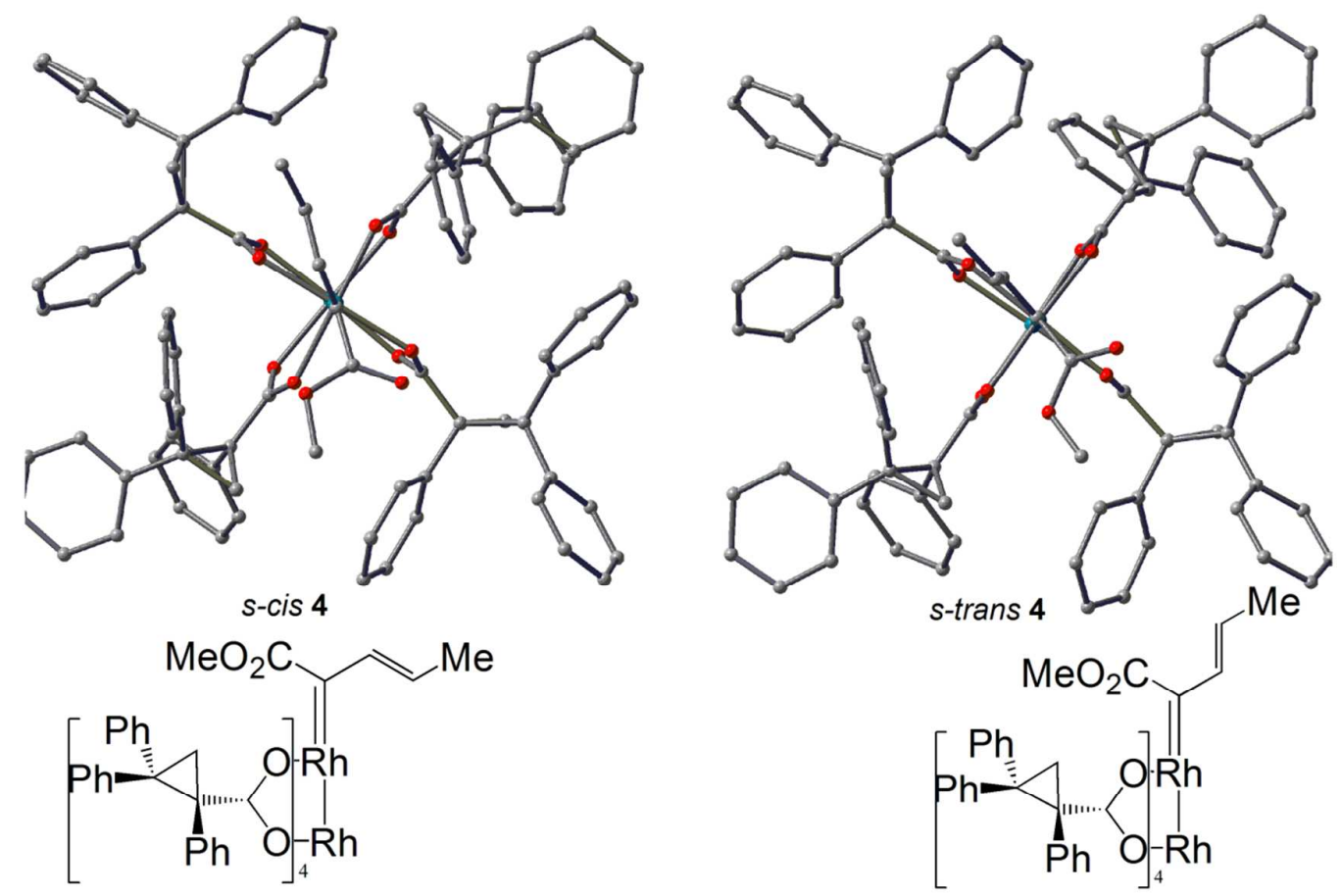

Figure S13. Optimized structures s-trans vinylcarbenoid $\mathbf{r}-4$ and $s$-cis $\mathbf{r}-\mathbf{4}$. Hydrogen atoms were omitted for clarity.

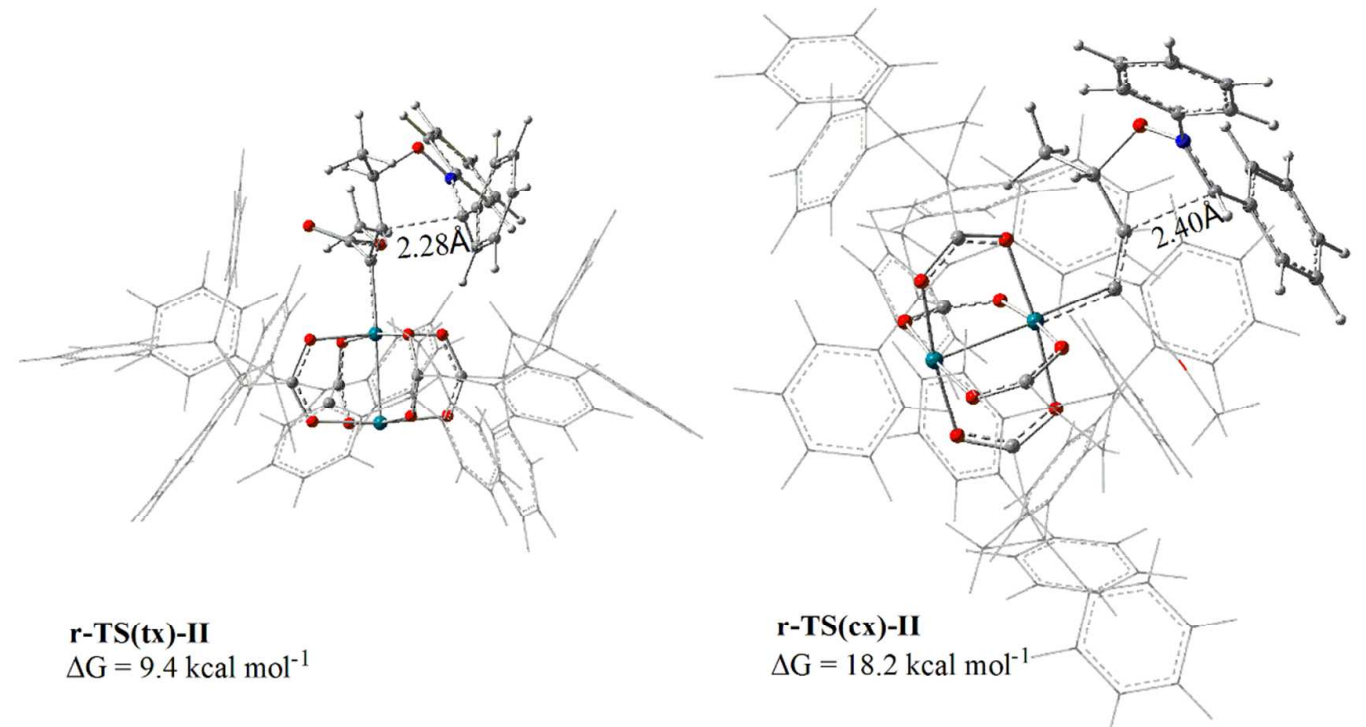

Figure S14. Transition structures $\mathbf{r}-\mathrm{TS}(\mathbf{t x})$-II and $\mathbf{r}$-TS(cx)-II for the $\mathrm{Rh}_{2} \mathrm{~L}_{4}$-catalyzed $[3+2]$-cycloaddition between vinylcarbenoid and nitrones. The Energies were calculated at the (SMD)M06-GD3/BS2//M06-GD3/BS1 level of theory, and are reported relative to $s$-cis vinycarbenoid $\mathbf{r}-\mathbf{4}+$ nitrone 2 . The bulky ligands on $\mathrm{Rh}_{2}(R-\mathrm{TPCP})_{4}$ are illustrated in wireframe for clarity. 
Part V. Coordinates and free energies ((SMD)M06-GD3/BS2//M06-GD3/BS1) of all stationary points.

Note: all the free energies in parentheses were calculated at the M06-GD3/BS2//M06-GD3-BS1 level of theory.

\begin{tabular}{|c|c|c|c|}
\hline & & & \\
\hline$c$ & 2.08425800 & -0.21303200 & $-0.09 / 89000$ \\
\hline $\mathrm{C}$ & 2.82075200 & 1.02418000 & 0.24725100 \\
\hline 0 & 2.74846200 & 1.93821600 & -0.71569400 \\
\hline $\mathrm{C}$ & 3.27545900 & 3.21958400 & -0.37057700 \\
\hline $\mathrm{H}$ & 4.33282400 & 3.14695700 & -0.09911300 \\
\hline $\mathrm{H}$ & 2.71810700 & 3.63446100 & 0.47494500 \\
\hline $\mathrm{H}$ & 3.14867600 & 3.84231100 & -1.25574500 \\
\hline 0 & 3.36652200 & 1.17340400 & 1.31906300 \\
\hline C & 4.10299900 & -1.58014900 & -0.05249900 \\
\hline $\mathrm{H}$ & 4.67780600 & -0.70280000 & 0.25093800 \\
\hline $\mathrm{Rh}$ & 0.13304200 & -0.11250100 & -0.06027300 \\
\hline 0 & 0.06906000 & -1.24258500 & 1.65953500 \\
\hline $\mathrm{C}$ & -1.05900800 & -1.49640800 & 2.18172900 \\
\hline $\mathrm{O}$ & -2.19749900 & -1.14926700 & 1.79417200 \\
\hline $\mathrm{Rh}$ & -2.33508100 & -0.01414800 & 0.07398000 \\
\hline 0 & -2.34883600 & -1.74506000 & -1.06405500 \\
\hline $\mathrm{C}$ & -1.25689000 & -2.25177900 & -1.40352200 \\
\hline 0 & -0.07876200 & -1.84629900 & -1.15485600 \\
\hline $\mathrm{H}$ & -1.30615700 & -3.17796300 & -2.00050900 \\
\hline 0 & -2.08525400 & 1.70723300 & 1.19660000 \\
\hline $\mathrm{C}$ & -0.92502500 & 2.12491600 & 1.41048800 \\
\hline 0 & 0.18161600 & 1.62809000 & 1.03652200 \\
\hline $\mathrm{H}$ & -0.83397900 & 3.05045700 & 2.00318200 \\
\hline $\mathrm{O}$ & -2.30183700 & 1.11433300 & -1.65221100 \\
\hline C & -1.18765700 & 1.35587700 & -2.17157400 \\
\hline 0 & -0.03494700 & 1.00242000 & -1.78110200 \\
\hline $\mathrm{H}$ & -1.19353600 & 1.95708800 & -3.09608100 \\
\hline $\mathrm{H}$ & -1.01191800 & -2.10639600 & 3.09908600 \\
\hline $\mathrm{C}$ & 2.76710800 & -1.44044500 & -0.26392500 \\
\hline $\mathrm{H}$ & 2.16621800 & -2.31228200 & -0.52910700 \\
\hline C & 4.83067000 & -2.86050700 & -0.15436200 \\
\hline $\mathrm{H}$ & 5.28477100 & -3.11680200 & 0.81213900 \\
\hline $\mathrm{H}$ & 4.17978400 & -3.68130100 & -0.46845600 \\
\hline $\mathrm{H}$ & 5.66494000 & -2.77180600 & -0.86258500 \\
\hline
\end{tabular}




$\begin{array}{cccc}\text { s-cis } 4(-1361.322697 a . u .) & & \\ \text { C } & -2.17849400 & 0.22596900 & -0.27971200 \\ \text { C } & -2.87198700 & -1.07372400 & -0.43429200 \\ \text { O } & -3.39001200 & -1.47815600 & 0.72899900 \\ \text { C } & -3.89553100 & -2.81387800 & 0.72825800 \\ \text { H } & -3.09468600 & -3.51521000 & 0.47313300 \\ \text { H } & -4.70832000 & -2.92136200 & 0.00417200 \\ \text { H } & -4.25626100 & -2.99897700 & 1.73996000 \\ \text { O } & -2.93894900 & -1.67379300 & -1.47999200 \\ \text { C } & -2.50952700 & 2.61280500 & 0.04062900 \\ \text { H } & -1.43443800 & 2.71443000 & 0.18093200 \\ \text { Rh } & -0.20737700 & 0.08955800 & -0.11722700 \\ \text { O } & -0.19849600 & 1.11317300 & 1.67827400 \\ \text { C } & 0.90796100 & 1.26186600 & 2.28335100 \\ \text { O } & 2.04643600 & 0.87434800 & 1.93954900 \\ \text { Rh } & 2.24032700 & -0.16249600 & 0.15952400 \\ \text { O } & 2.43539600 & 1.61893700 & -0.87354800 \\ \text { C } & 1.40040900 & 2.20451800 & -1.25926300 \\ \text { O } & 0.18665200 & 1.86283000 & -1.10023700 \\ \text { H } & 1.54321100 & 3.14843100 & -1.81192500 \\ \text { O } & 1.84315500 & -1.92959700 & 1.15163700 \\ \text { C } & -1.65187900 & -2.29848900 & 1.25819200 \\ \text { O } & -0.40081800 & -1.71418400 & 0.85812600 \\ \text { H } & 0.47643200 & -3.25969700 & 1.76964600 \\ \text { O } & 2.25295600 & -1.18379100 & -1.62889100 \\ \text { C } & 1.16002900 & -1.32660800 & -2.22745300 \\ \text { O } & 0.00925300 & -0.92377600 & -1.88557500 \\ \text { H } & 1.18786800 & -1.87414200 & -3.18377600 \\ \text { H } & 0.83943400 & 1.81027700 & 3.23805400 \\ \text { C } & -3.00279200 & 1.37204100 & -0.22969700 \\ \text { H } & -4.08426400 & 1.25800000 & -0.35985600 \\ \text { C } & -3.34339100 & 3.82187800 & 0.17105300 \\ \text { H } & -4.40504600 & 3.62220000 & -0.00095500 \\ \text { H } & -21939100 & 4.26149000 & 1.16956800 \\ \text { H } & 4.59051500 & -0.53543000\end{array}$

Nitrone 2 (-631.4723682 a.u.)

$\begin{array}{cccc}\mathrm{O} & -0.24744500 & -1.73770300 & -0.30267700 \\ \mathrm{~N} & -0.47429400 & -0.50767200 & -0.08985400 \\ \mathrm{C} & -1.87703000 & -0.12578000 & -0.04382700 \\ \mathrm{C} & -4.57318400 & 0.48724900 & 0.07363400 \\ \mathrm{C} & -2.77336100 & -1.07948500 & 0.42208300 \\ \mathrm{C} & -2.31341700 & 1.11937200 & -0.48319900 \\ & & \end{array}$




$\begin{array}{lrrr}\text { C } & -4.12283500 & -0.76310100 & 0.48866300 \\ \mathrm{C} & -3.66851200 & 1.42224500 & -0.41772300 \\ \mathrm{H} & -2.38744600 & -2.05069900 & 0.71451400 \\ \mathrm{H} & -1.61168900 & 1.83359900 & -0.90584000 \\ \mathrm{H} & -4.82870400 & -1.49998000 & 0.86332300 \\ \mathrm{H} & -4.01812100 & 2.38979800 & -0.76871500 \\ \mathrm{H} & -5.63218300 & 0.72816600 & 0.12047200 \\ \mathrm{C} & 1.87542400 & 0.22252200 & 0.07181400 \\ \mathrm{C} & 2.51464000 & -0.99432600 & -0.22424100 \\ \mathrm{C} & 2.66929600 & 1.35315100 & 0.33840300 \\ \mathrm{C} & 3.90133300 & -1.05754400 & -0.25260800 \\ \mathrm{H} & 1.90500400 & -1.86654000 & -0.42612800 \\ \mathrm{C} & 4.05112100 & 1.27806100 & 0.31032500 \\ \mathrm{H} & 2.18167100 & 2.29976700 & 0.56913000 \\ \mathrm{C} & 4.67439600 & 0.06800400 & 0.01294700 \\ \mathrm{H} & 4.38467800 & -2.00384300 & -0.48445600 \\ \mathrm{H} & 4.64596800 & 2.16388600 & 0.51969300 \\ \mathrm{H} & 5.75972800 & 0.00458800 & -0.01023700 \\ \mathrm{C} & 0.44737300 & 0.41254200 & 0.11905600 \\ \mathrm{H} & 0.06925200 & 1.39912200 & 0.36668600\end{array}$

TS(td)-I (-1992.790872 a.u.)

$\begin{array}{lrrr}\text { C } & -0.69562100 & -0.63800000 & 1.08107200 \\ \mathrm{C} & -1.48623500 & -1.78281300 & 0.58645300 \\ \mathrm{O} & -1.58047600 & -1.82369200 & -0.75759000 \\ \mathrm{C} & -1.97253400 & -3.09073800 & -1.27279100 \\ \mathrm{H} & -2.98401500 & -3.35782800 & -0.94644200 \\ \mathrm{H} & -1.27262400 & -3.86122100 & -0.93503300 \\ \mathrm{H} & -1.93163300 & -3.00109000 & -2.36065900 \\ \mathrm{O} & -1.92710600 & -2.65412600 & 1.30644500 \\ \mathrm{O} & -3.18707900 & 1.78904200 & 1.17403100 \\ \mathrm{~N} & -2.72264400 & 1.67004000 & -0.04961600 \\ \mathrm{C} & -1.69983400 & 2.60116900 & -0.44014800 \\ \mathrm{C} & 0.31801400 & 4.35735900 & -1.15829500 \\ \mathrm{C} & -0.94595600 & 3.21646600 & 0.55114800 \\ \mathrm{C} & -1.49417100 & 2.89759800 & -1.78770300 \\ \mathrm{C} & 0.06823200 & 4.09036900 & 0.18267400 \\ \mathrm{C} & -0.47542700 & 3.76896100 & -2.14132900 \\ \mathrm{H} & -1.14973700 & 2.99678500 & 1.59228200 \\ \mathrm{H} & -2.13484000 & 2.47422200 & -2.55669700 \\ \mathrm{H} & 0.67017400 & 4.56154200 & 0.95546900 \\ \mathrm{H} & -0.31000100 & 4.00234900 & -3.19009400 \\ \mathrm{C} & -2.55886000 & 0.40204300 & 2.30602500 \\ \mathrm{H} & -3.22289700 & -0.38211200 & 1.93751300 \\ & & & \\ & & & \end{array}$




$\begin{array}{lrrr}\text { H } & 1.11677100 & 5.03838900 & -1.43986100 \\ \text { Rh } & 1.21134900 & -0.51754100 & 0.40744400 \\ \text { O } & 2.03500800 & -1.40836500 & 2.06301400 \\ \text { C } & 3.29203600 & -1.56085400 & 2.09398700 \\ \text { O } & 4.15073400 & -1.22801700 & 1.24283600 \\ \text { Rh } & 3.52107500 & -0.27018200 & -0.48166700 \\ \text { O } & 3.60159100 & 1.54863800 & 0.50659200 \\ \text { C } & 2.59909900 & 1.91323300 & 1.16072300 \\ \text { O } & 1.48491300 & 1.32965800 & 1.31324700 \\ \text { H } & 2.68705000 & 2.88309400 & 1.68426700 \\ \text { O } & 3.24554800 & -2.11244300 & -1.38599800 \\ \mathrm{C} & 2.16845100 & -2.72178800 & -1.19110600 \\ \text { O } & 1.15352500 & -2.36033000 & -0.52575100 \\ \text { H } & 2.07800300 & -3.71555300 & -1.66468100 \\ \text { O } & 2.67353700 & 0.68004300 & -2.12175900 \\ \mathrm{C} & 1.42908400 & 0.81283500 & -2.15666700 \\ \mathrm{O} & 0.55669900 & 0.43363000 & -1.32029200 \\ \mathrm{H} & 1.02002200 & 1.33587900 & -3.04134900 \\ \mathrm{H} & 3.67154900 & -2.05710200 & 3.00380100 \\ \mathrm{C} & -1.18598200 & 0.25938000 & 1.98495800 \\ \mathrm{H} & -0.49013300 & 0.97651600 & 2.42530600 \\ \mathrm{C} & -2.98146700 & 1.06798800 & 3.56562500 \\ \mathrm{H} & -2.88893600 & 0.34501500 & 4.38544500 \\ \mathrm{H} & -2.33932100 & 1.92501400 & 3.79451000 \\ \mathrm{H} & -4.02281800 & 1.40120800 & 3.52076800 \\ \mathrm{C} & -4.29738400 & -0.11607900 & -0.70484400 \\ \mathrm{C} & -5.27160600 & 0.02420900 & 0.30199200 \\ \mathrm{C} & -4.49403700 & -1.08131900 & -1.70836200 \\ \mathrm{C} & -6.39403900 & -0.78831300 & 0.29562400 \\ \mathrm{H} & -5.13900500 & 0.78107000 & 1.06765300 \\ \mathrm{C} & -5.61279100 & -1.89769100 & -1.69975500 \\ \mathrm{H} & -3.74675900 & -1.18355700 & -2.49341200 \\ \mathrm{H} & -6.56476900 & -1.75469600 & -0.69343500 \\ \mathrm{H} & -7.14257400 & -0.67133300 & 1.07496900 \\ \mathrm{H} & -5.74433700 & -2.64513400 & -2.47787200 \\ \mathrm{H} & -2.39649900 & -0.68049200 \\ \mathrm{H} & -12998300 & 0.71602700 & -0.85281600 \\ \mathrm{H} & 0.58323200 & -1.74439000\end{array}$

(td)-A (-1992.794818 a.u.)

$\begin{array}{lllr}\text { C } & -0.67373700 & -0.70535100 & 1.04052500 \\ \text { C } & -1.45361200 & -1.78388000 & 0.41633700 \\ \text { O } & -1.54925000 & -1.65192900 & -0.93459700 \\ \text { C } & -1.90703000 & -2.85126800 & -1.60677800 \\ & & \end{array}$




\begin{tabular}{|c|c|c|c|}
\hline $\mathrm{H}$ & -2.91303100 & -3.18636900 & -1.32568200 \\
\hline $\mathrm{H}$ & -1.19263400 & -3.64351400 & -1.36266800 \\
\hline $\mathrm{H}$ & -1.86075600 & -2.62729200 & -2.67574900 \\
\hline $\mathrm{O}$ & -1.91184800 & -2.74273700 & 1.00081000 \\
\hline $\mathrm{O}$ & -3.10555500 & 1.59163000 & 1.34783800 \\
\hline$N$ & -2.72942800 & 1.55489400 & 0.04782900 \\
\hline C & -1.77442600 & 2.54116200 & -0.35224400 \\
\hline C & 0.11006100 & 4.39939800 & -1.16320800 \\
\hline C & -0.88993800 & 3.06616500 & 0.58327900 \\
\hline C & -1.76360800 & 2.97618000 & -1.67913200 \\
\hline C & 0.05528700 & 3.99312000 & 0.16490300 \\
\hline C & -0.80822000 & 3.89543300 & -2.08258700 \\
\hline $\mathrm{H}$ & -0.92337900 & 2.73225100 & 1.61265200 \\
\hline $\mathrm{H}$ & -2.51028500 & 2.62509600 & -2.38680300 \\
\hline $\mathrm{H}$ & 0.76116800 & 4.39167900 & 0.88836100 \\
\hline $\mathrm{H}$ & -0.79415300 & 4.23578500 & -3.11452900 \\
\hline C & -2.62246200 & 0.36446500 & 2.16977500 \\
\hline $\mathrm{H}$ & -3.22208900 & -0.48428300 & 1.81809800 \\
\hline $\mathrm{H}$ & 0.85781000 & 5.12083100 & -1.48155400 \\
\hline $\mathrm{Rh}$ & 1.26794100 & -0.53491300 & 0.39433600 \\
\hline $\mathrm{O}$ & 2.08254400 & -1.57785800 & 1.96440000 \\
\hline C & 3.33824800 & -1.73413100 & 1.98322100 \\
\hline $\mathrm{O}$ & 4.20199100 & -1.32361600 & 1.17045700 \\
\hline $\mathrm{Rh}$ & 3.58286500 & -0.20444100 & -0.45953900 \\
\hline $\mathrm{O}$ & 3.66184900 & 1.51192100 & 0.70305400 \\
\hline C & 2.65478000 & 1.81591300 & 1.38098300 \\
\hline 0 & 1.53525400 & 1.22947400 & 1.46462000 \\
\hline $\mathrm{H}$ & 2.74493000 & 2.72959600 & 1.99863400 \\
\hline $\mathrm{O}$ & 3.30343600 & -1.95319100 & -1.53457500 \\
\hline C & 2.22418800 & -2.57641900 & -1.40349300 \\
\hline $\mathrm{O}$ & 1.20706700 & -2.28064000 & -0.71068400 \\
\hline $\mathrm{H}$ & 2.13560100 & -3.51940000 & -1.97275300 \\
\hline $\mathrm{O}$ & 2.73161800 & 0.89686700 & -2.00605300 \\
\hline C & 1.48591400 & 1.02369300 & -2.03811000 \\
\hline $\mathrm{O}$ & 0.60970300 & 0.56748700 & -1.24663200 \\
\hline $\mathrm{H}$ & 1.08100500 & 1.62108000 & -2.87758900 \\
\hline $\mathrm{H}$ & 3.71624200 & -2.31549100 & 2.84244000 \\
\hline C & -1.18613700 & 0.15542500 & 1.93539000 \\
\hline $\mathrm{H}$ & -0.50956200 & 0.84367000 & 2.44811600 \\
\hline C & -3.00756500 & 0.78661600 & 3.56028600 \\
\hline $\mathrm{H}$ & -2.79520900 & -0.03695000 & 4.24838400 \\
\hline $\mathrm{H}$ & -2.41733700 & 1.65628400 & 3.87161200 \\
\hline $\mathrm{H}$ & -4.07107700 & 1.03971500 & 3.63299000 \\
\hline C & -4.40163500 & -0.14636600 & -0.61314000 \\
\hline
\end{tabular}




$\begin{array}{cccc}\text { C } & -5.33715300 & -0.01099100 & 0.43098400 \\ \mathrm{C} & -4.65445600 & -1.07096600 & -1.64197000 \\ \mathrm{C} & -6.47742200 & -0.79519200 & 0.43948000 \\ \mathrm{H} & -5.16713400 & 0.72070300 & 1.21406500 \\ \mathrm{C} & -5.78995100 & -1.86339100 & -1.61639900 \\ \mathrm{H} & -3.94236900 & -1.15910200 & -2.46000800 \\ \mathrm{C} & -6.70061700 & -1.72893100 & -0.57190700 \\ \mathrm{H} & -7.19788900 & -0.68423700 & 1.24519400 \\ \mathrm{H} & -5.96723000 & -2.58319400 & -2.41071600 \\ \mathrm{H} & -7.59165900 & -2.35124400 & -0.54683900 \\ \mathrm{C} & -3.22871000 & 0.66998700 & -0.77600500 \\ \mathrm{H} & -2.68654500 & 0.59778100 & -1.71654700\end{array}$

TS(td)-II (-1992.787088 a.u.)

$\begin{array}{lrrr}\text { C } & -0.63983700 & -0.82110100 & 0.99776000 \\ \mathrm{C} & -0.94851800 & -2.22389400 & 1.36205500 \\ \mathrm{O} & -0.59470400 & -3.09685000 & 0.41777600 \\ \mathrm{C} & -0.90454500 & -4.45454700 & 0.70630300 \\ \mathrm{H} & -1.97618700 & -4.57232500 & 0.90335500 \\ \mathrm{H} & -0.34471900 & -4.79811300 & 1.58121800 \\ \mathrm{H} & -0.61605400 & -5.02301200 & -0.17898900 \\ \mathrm{O} & -1.44910700 & -2.54093000 & 2.42383700 \\ \mathrm{O} & -3.91052400 & 0.77214500 & 0.97743900 \\ \mathrm{~N} & -3.34516800 & 1.14990100 & -0.21679100 \\ \mathrm{C} & -3.03916700 & 2.52997600 & -0.32977700 \\ \mathrm{C} & -2.36386200 & 5.21155200 & -0.60180400 \\ \mathrm{C} & -3.14601700 & 3.38101100 & 0.76835200 \\ \mathrm{C} & -2.63850700 & 3.02105100 & -1.57445000 \\ \mathrm{C} & -2.80496500 & 4.72024200 & 0.62254000 \\ \mathrm{C} & -2.28679800 & 4.35776800 & -1.69927900 \\ \mathrm{H} & -3.49565500 & 2.99047500 & 1.71830000 \\ \mathrm{H} & -2.61505400 & 2.36248700 & -2.43982200 \\ \mathrm{H} & -2.88057300 & 5.38333300 & 1.48057500 \\ \mathrm{H} & -1.96710000 & 4.73740400 & -2.66663700 \\ \mathrm{C} & -2.94008800 & -0.01152900 & 1.81007600 \\ \mathrm{H} & -3.27675700 & -1.05501700 & 1.76423500 \\ \mathrm{H} & -2.09397700 & 6.25908600 & -0.70553500 \\ \mathrm{Rh} & 1.16723700 & -0.26683900 & 0.30742300 \\ \mathrm{O} & 1.40606700 & 0.96160100 & 1.94086100 \\ \mathrm{C} & 2.48336500 & 1.62223700 & 2.04603700 \\ \mathrm{O} & 3.46573200 & 1.65645200 & 1.27020400 \\ \mathrm{Rh} & 3.39918500 & 0.51759600 & -0.45659600 \\ \mathrm{O} & 2.39629300 & 2.06476700 & -1.42230700 \\ \mathrm{C} & 1.15003000 & 2.11300000 & -1.35705600 \\ & & & \\ & 528 & \\ & & & \\ & & & \\ & & & \\ & & & \end{array}$




\begin{tabular}{lrrr}
$\mathrm{O}$ & 0.35070800 & 1.32307900 & -0.76212400 \\
$\mathrm{H}$ & 0.65772200 & 2.95087800 & -1.88342700 \\
$\mathrm{O}$ & 4.17381400 & -1.09465400 & 0.58556600 \\
$\mathrm{C}$ & 3.38812300 & -1.85199300 & 1.20119000 \\
$\mathrm{O}$ & 2.12486500 & -1.79176100 & 1.29016700 \\
$\mathrm{H}$ & 3.84574800 & -2.69510100 & 1.74650900 \\
$\mathrm{O}$ & 3.15191700 & -0.68885800 & -2.11874500 \\
$\mathrm{C}$ & 2.09055700 & -1.34348500 & -2.22546900 \\
$\mathrm{O}$ & 1.09637400 & -1.39025100 & -1.44001400 \\
$\mathrm{H}$ & 1.99393200 & -1.97118000 & -3.12917200 \\
$\mathrm{H}$ & 2.54917000 & 2.25075000 & 2.95077100 \\
$\mathrm{C}$ & -1.58740000 & 0.14599000 & 1.20658500 \\
$\mathrm{H}$ & -1.23822300 & 1.18184300 & 1.13510600 \\
$\mathrm{C}$ & -3.06055300 & 0.51493200 & 3.21848200 \\
$\mathrm{H}$ & -2.53964500 & -0.17267500 & 3.89178800 \\
$\mathrm{H}$ & -2.60200700 & 1.50605500 & 3.31260900 \\
$\mathrm{H}$ & -4.11114400 & 0.57449200 & 3.52163100 \\
$\mathrm{C}$ & -2.98904600 & -1.14228100 & -1.08313900 \\
$\mathrm{C}$ & -4.23512600 & -1.66021900 & -0.69569000 \\
$\mathrm{C}$ & -2.12291000 & -1.92736200 & -1.85742700 \\
$\mathrm{C}$ & -4.60106200 & -2.93920800 & -1.08711300 \\
$\mathrm{H}$ & -4.91027700 & -1.05005000 & -0.10139300 \\
$\mathrm{C}$ & -2.49966900 & -3.20408200 & -2.24633000 \\
$\mathrm{H}$ & -1.13481600 & -1.54430100 & -2.11222600 \\
$\mathrm{C}$ & -3.73722400 & -3.71221400 & -1.86220700 \\
$\mathrm{H}$ & -5.56830500 & -3.33590800 & -0.78931900 \\
$\mathrm{H}$ & -1.81813600 & -3.80734200 & -2.84093000 \\
$\mathrm{H}$ & -4.03125800 & -4.71489800 & -2.16401100 \\
$\mathrm{C}$ & -2.54711800 & 0.20830300 & -0.77257700 \\
$\mathrm{H}$ & -1.70898200 & 0.60693600 & -1.34795700 \\
& & & \\
\hline & & & \\
\hline & & &
\end{tabular}

(td)-B (-1992.800264 a.u.)

$\begin{array}{lccc}\mathrm{C} & 0.53859200 & 0.87355200 & 0.74644500 \\ \mathrm{C} & 0.55888500 & 2.20659700 & 1.38384500 \\ \mathrm{O} & 0.39310500 & 3.22182700 & 0.55549600 \\ \mathrm{C} & 0.33474000 & 4.51044300 & 1.16884600 \\ \mathrm{H} & 1.23740100 & 4.69464000 & 1.75890800 \\ \mathrm{H} & -0.54309500 & 4.57673600 & 1.81842300 \\ \mathrm{H} & 0.26341800 & 5.22395700 & 0.34815700 \\ \mathrm{O} & 0.65296800 & 2.27445400 & 2.59271400 \\ \mathrm{O} & 4.02344500 & -0.22255600 & 1.10688900 \\ \mathrm{~N} & 3.70610400 & -0.64951600 & -0.20025200 \\ \mathrm{C} & 3.61613800 & -2.06602800 & -0.28428700 \\ \mathrm{C} & 3.44668300 & -4.84700000 & -0.54352200 \\ & & & \end{array}$




\begin{tabular}{|c|c|c|c|}
\hline C & 3.95522900 & -2.89219500 & 0.78835400 \\
\hline C & 3.22827200 & -2.64077800 & -1.50142300 \\
\hline C & 3.86677800 & -4.27353200 & 0.65082800 \\
\hline C & 3.13370400 & -4.02000800 & -1.61962900 \\
\hline $\mathrm{H}$ & 4.29396900 & -2.44475300 & 1.71664000 \\
\hline $\mathrm{H}$ & 3.02028300 & -2.00629700 & -2.36044900 \\
\hline $\mathrm{H}$ & 4.12958000 & -4.90608100 & 1.49581500 \\
\hline $\mathrm{H}$ & 2.82476200 & -4.45174400 & -2.56910500 \\
\hline C & 2.83598200 & 0.28663000 & 1.77130900 \\
\hline $\mathrm{H}$ & 2.98700300 & 1.36968400 & 1.89684500 \\
\hline $\mathrm{H}$ & 3.37608300 & -5.92713200 & -0.64158000 \\
\hline $\mathrm{Rh}$ & -1.17027700 & 0.14176200 & 0.18215100 \\
\hline $\mathrm{O}$ & -1.07120100 & -1.18904100 & 1.75113600 \\
\hline C & -2.03454500 & -1.99699500 & 1.93161500 \\
\hline $\mathrm{O}$ & -3.08498100 & -2.12075900 & 1.26412600 \\
\hline $\mathrm{Rh}$ & -3.34518100 & -0.89638800 & -0.37788300 \\
\hline 0 & -2.27738700 & -2.24640000 & -1.53428500 \\
\hline C & -1.03385800 & -2.13597500 & -1.59482900 \\
\hline $\mathrm{O}$ & -0.28572800 & -1.27158800 & -1.03796300 \\
\hline $\mathrm{H}$ & -0.49441900 & -2.87769000 & -2.20779500 \\
\hline $\mathrm{O}$ & -4.20560300 & 0.54198000 & 0.83481000 \\
\hline C & -3.46523100 & 1.38056000 & 1.39548100 \\
\hline $\mathrm{O}$ & -2.20120300 & 1.49020200 & 1.33842600 \\
\hline $\mathrm{H}$ & -3.96054500 & 2.13444100 & 2.02933800 \\
\hline $\mathrm{O}$ & -3.45384400 & 0.40357000 & -1.97748100 \\
\hline C & -2.51410800 & 1.21278800 & -2.14452100 \\
\hline $\mathrm{O}$ & -1.45585200 & 1.35548300 & -1.45844600 \\
\hline $\mathrm{H}$ & -2.59995900 & 1.90153400 & -3.00151700 \\
\hline $\mathrm{H}$ & -1.90975000 & -2.67107500 & 2.79474500 \\
\hline C & 1.71267900 & 0.01561600 & 0.78027800 \\
\hline $\mathrm{H}$ & 1.38689500 & -1.03435300 & 0.85846200 \\
\hline C & 2.64982700 & -0.37267700 & 3.11516000 \\
\hline $\mathrm{H}$ & 1.83214200 & 0.12211000 & 3.65072400 \\
\hline $\mathrm{H}$ & 2.40391000 & -1.43456500 & 2.99778100 \\
\hline $\mathrm{H}$ & 3.56208600 & -0.28611600 & 3.71419400 \\
\hline C & 2.74119100 & 1.48007400 & -1.07857700 \\
\hline C & 3.80661600 & 2.27247900 & -0.64818100 \\
\hline C & 1.79870300 & 2.01340600 & -1.95892600 \\
\hline C & 3.90907800 & 3.59035300 & -1.08167800 \\
\hline $\mathrm{H}$ & 4.55361300 & 1.84727200 & 0.01888000 \\
\hline C & 1.90367400 & 3.33056600 & -2.38991000 \\
\hline $\mathrm{H}$ & 0.95732900 & 1.39993900 & -2.28260100 \\
\hline C & 2.95730900 & 4.12344400 & -1.94760000 \\
\hline $\mathrm{H}$ & 4.74187100 & 4.20336200 & -0.74420800 \\
\hline
\end{tabular}




$\begin{array}{lrrr}\mathrm{H} & 1.15736600 & 3.73595700 & -3.06919200 \\ \mathrm{H} & 3.04420600 & 5.15435200 & -2.28385600 \\ \mathrm{C} & 2.51449400 & 0.07808500 & -0.58608900 \\ \mathrm{H} & 1.95398000 & -0.49318000 & -1.33022700\end{array}$

TS(td)-III (-1992.712519 a.u.)

\begin{tabular}{|c|c|c|c|}
\hline C & 0.30607000 & 0.73624100 & 0.48947100 \\
\hline C & 0.45257800 & 2.19336600 & 0.74298000 \\
\hline $\mathrm{O}$ & 0.11132700 & 2.96681100 & -0.28270300 \\
\hline C & 0.20698700 & 4.36376700 & -0.02922200 \\
\hline $\mathrm{H}$ & 1.22510300 & 4.62455100 & 0.28294700 \\
\hline $\mathrm{H}$ & -0.49459200 & 4.65688000 & 0.75777400 \\
\hline $\mathrm{H}$ & -0.04375000 & 4.85771400 & -0.96851000 \\
\hline $\mathrm{O}$ & 0.77866700 & 2.59903300 & 1.84117100 \\
\hline 0 & 2.94937300 & -1.42116100 & 1.85936300 \\
\hline $\mathrm{N}$ & 3.20388100 & -1.02992000 & 0.53110300 \\
\hline C & 4.18278300 & -1.74526000 & -0.15071400 \\
\hline C & 6.16888000 & -3.08426200 & -1.55644700 \\
\hline C & 5.09404000 & -2.54674700 & 0.54114900 \\
\hline C & 4.24655600 & -1.62441500 & -1.54307000 \\
\hline C & 6.07988900 & -3.21387600 & -0.17345300 \\
\hline C & 5.24942100 & -2.28648200 & -2.23414100 \\
\hline $\mathrm{H}$ & 5.02095200 & -2.63157000 & 1.62027000 \\
\hline $\mathrm{H}$ & 3.50575100 & -1.02378500 & -2.06652000 \\
\hline $\mathrm{H}$ & 6.79039800 & -3.83951000 & 0.36134600 \\
\hline $\mathrm{H}$ & 5.30185400 & -2.19090600 & -3.31580400 \\
\hline C & 2.06989700 & -0.38319800 & 2.43717800 \\
\hline $\mathrm{H}$ & 2.68113000 & 0.48723300 & 2.71690500 \\
\hline $\mathrm{H}$ & 6.94577900 & -3.60913900 & -2.10603400 \\
\hline $\mathrm{Rh}$ & -1.50739900 & 0.04631600 & 0.02163600 \\
\hline 0 & -1.63432300 & -0.84838700 & 1.87695600 \\
\hline C & -2.69616300 & -1.48089900 & 2.16511200 \\
\hline $\mathrm{O}$ & -3.71942600 & -1.65768700 & 1.46667700 \\
\hline $\mathrm{Rh}$ & -3.76543400 & -0.84824400 & -0.43973700 \\
\hline 0 & -2.81028700 & -2.53661000 & -1.17377700 \\
\hline C & -1.56020300 & -2.58223700 & -1.16005800 \\
\hline 0 & -0.73835900 & -1.70753400 & -0.74429800 \\
\hline $\mathrm{H}$ & -1.09398100 & -3.49894700 & -1.55899600 \\
\hline $\mathrm{O}$ & -4.51971800 & 0.91308500 & 0.33420000 \\
\hline C & -3.70730600 & 1.77388900 & 0.74501900 \\
\hline $\mathrm{O}$ & -2.44069300 & 1.73199200 & 0.75813300 \\
\hline $\mathrm{H}$ & -4.14079800 & 2.70196400 & 1.15469700 \\
\hline $\mathrm{O}$ & -3.60874400 & 0.04308500 & -2.30053400 \\
\hline C & -2.55585700 & 0.66080400 & -2.58503600 \\
\hline
\end{tabular}




$\begin{array}{lrrr}\mathrm{O} & -1.52133200 & 0.84162000 & -1.87572000 \\ \mathrm{H} & -2.51003300 & 1.11396400 & -3.59001900 \\ \mathrm{H} & -2.70310600 & -1.93241400 & 3.17210400 \\ \mathrm{C} & 1.27802600 & -0.15513400 & 1.17941000 \\ \mathrm{H} & 0.88302500 & -1.16218500 & 0.96363100 \\ \mathrm{C} & 1.36046500 & -0.99071400 & 3.60992600 \\ \mathrm{H} & 0.68307500 & -0.24125400 & 4.03206300 \\ \mathrm{H} & 0.76384300 & -1.85084600 & 3.28922100 \\ \mathrm{H} & 2.06572100 & -1.30732300 & 4.38407000 \\ \mathrm{C} & 3.07637600 & 1.26662800 & -0.23251400 \\ \mathrm{C} & 4.16351800 & 1.70114400 & 0.53730800 \\ \mathrm{C} & 2.69175900 & 2.01400600 & -1.34615600 \\ \mathrm{C} & 4.80491100 & 2.89323800 & 0.23505200 \\ \mathrm{H} & 4.49945400 & 1.09890500 & 1.37980800 \\ \mathrm{C} & 3.33696500 & 3.20855200 & -1.64948400 \\ \mathrm{H} & 1.87285100 & 1.65949800 & -1.97171900 \\ \mathrm{C} & 4.38846300 & 3.65338200 & -0.85593300 \\ \mathrm{H} & 5.63374800 & 3.23116400 & 0.85214600 \\ \mathrm{H} & 3.02152300 & 3.78448600 & -2.51629900 \\ \mathrm{H} & 4.89556600 & 4.58520900 & -1.09497100 \\ \mathrm{C} & 2.37779700 & -0.00337400 & 0.15027500 \\ \mathrm{H} & 1.17979800 & 0.17806600 & -0.65695100\end{array}$

TS(tx)-I (-1992.794374 a.u.)

$\begin{array}{lccc}\text { C } & -0.70746300 & -0.36486300 & 1.12097200 \\ \text { C } & -1.45739300 & -1.63305900 & 0.98595400 \\ \text { O } & -1.67823000 & -1.95188300 & -0.29813700 \\ \text { C } & -2.17974300 & -3.26765900 & -0.50799000 \\ H & -3.11182000 & -3.42516000 & 0.04496600 \\ H & -1.43877800 & -4.00310500 & -0.17905400 \\ H & -2.35548800 & -3.35461600 & -1.58137900 \\ \text { O } & -1.78078800 & -2.33564100 & 1.92062000 \\ \text { O } & -3.67984000 & 1.40506600 & 0.97337100 \\ \text { N } & -3.35389300 & 0.84898700 & -0.16292500 \\ \text { C } & -4.15198800 & -0.27897900 & -0.57241400 \\ \text { C } & -5.73793500 & -2.43316700 & -1.31148500 \\ \text { C } & -4.89052100 & -0.95230600 & 0.39529900 \\ \text { C } & -4.22925200 & -0.64865400 & -1.91386700 \\ \text { C } & -5.67594700 & -2.03530900 & 0.02031200 \\ \text { C } & -5.01654900 & -1.73386900 & -2.27523400 \\ H & -4.85538400 & -0.61468500 & 1.42515700 \\ H & -3.70321900 & -0.08421500 & -2.67868100 \\ H & -6.24597200 & -2.56684600 & 0.77770200 \\ H & -5.07981600 & -2.02097900 & -3.32163900 \\ & & & \\ & & 532 & \end{array}$




\begin{tabular}{|c|c|c|c|}
\hline C & -2.48592300 & 0.73632700 & 2.40277400 \\
\hline $\mathrm{H}$ & -3.01289100 & -0.21591200 & 2.46833500 \\
\hline $\mathrm{H}$ & -6.35821800 & -3.27733800 & -1.60106200 \\
\hline $\mathrm{Rh}$ & 1.16325500 & -0.39398500 & 0.37831100 \\
\hline $\mathrm{O}$ & 1.95511400 & -0.50350300 & 2.26803000 \\
\hline C & 3.21636600 & -0.52032300 & 2.39000300 \\
\hline $\mathrm{O}$ & 4.09640000 & -0.49165000 & 1.49811200 \\
\hline $\mathrm{Rh}$ & 3.49507100 & -0.42408400 & -0.47941000 \\
\hline $\mathrm{O}$ & 3.43355500 & 1.64908100 & -0.37752100 \\
\hline C & 2.40937400 & 2.19604200 & 0.09125900 \\
\hline $\mathrm{O}$ & 1.32945600 & 1.66411600 & 0.48954500 \\
\hline $\mathrm{H}$ & 2.43385300 & 3.29776200 & 0.17092900 \\
\hline $\mathrm{O}$ & 3.33268500 & -2.49004500 & -0.48760700 \\
\hline C & 2.24811700 & -3.01266200 & -0.14088300 \\
\hline $\mathrm{O}$ & 1.17604500 & -2.45268800 & 0.23711400 \\
\hline $\mathrm{H}$ & 2.20636600 & -4.11548600 & -0.16679500 \\
\hline $\mathrm{O}$ & 2.68912200 & -0.35927200 & -2.38977200 \\
\hline C & 1.44622400 & -0.29262600 & -2.51014700 \\
\hline $\mathrm{O}$ & 0.55278800 & -0.26206200 & -1.61018000 \\
\hline $\mathrm{H}$ & 1.05908000 & -0.24791100 & -3.54540200 \\
\hline $\mathrm{H}$ & 3.57497400 & -0.56587800 & 3.43252900 \\
\hline C & -1.19686200 & 0.69901400 & 1.83633900 \\
\hline $\mathrm{H}$ & -0.56547600 & 1.58495200 & 1.93649100 \\
\hline C & -2.86134500 & 1.77042500 & 3.39904800 \\
\hline $\mathrm{H}$ & -2.56760000 & 1.42177800 & 4.39728000 \\
\hline $\mathrm{H}$ & -2.34233500 & 2.71511400 & 3.20554200 \\
\hline $\mathrm{H}$ & -3.94171000 & 1.94175700 & 3.40807800 \\
\hline C & -1.63755500 & 2.52828700 & -0.77452700 \\
\hline C & -1.97020200 & 3.56598500 & 0.11535800 \\
\hline C & -0.61385600 & 2.73805500 & -1.71420000 \\
\hline C & -1.28336700 & 4.76742700 & 0.05915100 \\
\hline $\mathrm{H}$ & -2.76742300 & 3.41173300 & 0.83413900 \\
\hline C & 0.06430200 & 3.94489300 & -1.76697100 \\
\hline $\mathrm{H}$ & -0.34344100 & 1.92458000 & -2.38313100 \\
\hline C & -0.26429000 & 4.96022600 & -0.87362700 \\
\hline $\mathrm{H}$ & -1.54410600 & 5.56567600 & 0.74967900 \\
\hline $\mathrm{H}$ & 0.85809900 & 4.08856100 & -2.49592800 \\
\hline $\mathrm{H}$ & 0.27175200 & 5.90605000 & -0.90393300 \\
\hline C & -2.31274400 & 1.25856600 & -0.86163500 \\
\hline $\mathrm{H}$ & -1.99704900 & 0.58154300 & -1.65344600 \\
\hline
\end{tabular}

(tx)-A (-1992.801517 a.u.)

$\begin{array}{llll}\text { C } & -0.71027000 & -0.38071000 & 1.04474200 \\ \text { C } & -1.43586100 & -1.65000500 & 0.84930900\end{array}$




\begin{tabular}{|c|c|c|c|}
\hline $\mathrm{O}$ & -1.67350600 & -1.89058200 & -0.45799200 \\
\hline $\mathrm{C}$ & -2.18193700 & -3.18897800 & -0.73833600 \\
\hline $\mathrm{H}$ & -3.15693000 & -3.34132100 & -0.26014100 \\
\hline $\mathrm{H}$ & -1.48393900 & -3.95026300 & -0.37670500 \\
\hline $\mathrm{H}$ & -2.28032200 & -3.24755700 & -1.82380900 \\
\hline $\mathrm{O}$ & -1.75474600 & -2.41905800 & 1.73125600 \\
\hline $\mathrm{O}$ & -3.60399200 & 1.30925200 & 1.15471300 \\
\hline $\mathrm{N}$ & -3.38868900 & 0.78737600 & -0.07221400 \\
\hline C & -4.24028900 & -0.28775200 & -0.46773100 \\
\hline C & -5.91037100 & -2.36483000 & -1.24577500 \\
\hline C & -4.96103100 & -0.98788200 & 0.49676000 \\
\hline C & -4.38337300 & -0.58936000 & -1.82271500 \\
\hline C & -5.78919600 & -2.03010200 & 0.09900500 \\
\hline C & -5.20863800 & -1.63774100 & -2.20339900 \\
\hline $\mathrm{H}$ & -4.88355500 & -0.71511900 & 1.54304900 \\
\hline $\mathrm{H}$ & -3.87449100 & 0.00141400 & -2.57964100 \\
\hline $\mathrm{H}$ & -6.34410500 & -2.58217600 & 0.85261800 \\
\hline $\mathrm{H}$ & -5.31776400 & -1.87304900 & -3.25875300 \\
\hline C & -2.63360100 & 0.70328100 & 2.20881100 \\
\hline $\mathrm{H}$ & -3.01442200 & -0.31166400 & 2.37627300 \\
\hline $\mathrm{H}$ & -6.56215600 & -3.17969300 & -1.54902200 \\
\hline $\mathrm{Rh}$ & 1.20579800 & -0.38727700 & 0.35295300 \\
\hline $\mathrm{O}$ & 1.93330300 & -0.50764300 & 2.26688300 \\
\hline C & 3.18933900 & -0.51122600 & 2.42810100 \\
\hline $\mathrm{O}$ & 4.09863600 & -0.46414200 & 1.56579700 \\
\hline $\mathrm{Rh}$ & 3.56597300 & -0.39406500 & -0.43247800 \\
\hline $\mathrm{O}$ & 3.48019600 & 1.68122900 & -0.32274500 \\
\hline C & 2.43670900 & 2.21722800 & 0.11482100 \\
\hline $\mathrm{O}$ & 1.34851700 & 1.67731700 & 0.47716500 \\
\hline $\mathrm{H}$ & 2.45093800 & 3.31997800 & 0.19575400 \\
\hline $\mathrm{O}$ & 3.41557400 & -2.46208800 & -0.45236900 \\
\hline C & 2.32073100 & -2.99313100 & -0.15188100 \\
\hline $\mathrm{O}$ & 1.23196100 & -2.44301200 & 0.18931200 \\
\hline $\mathrm{H}$ & 2.28712000 & -4.09613300 & -0.18894700 \\
\hline $\mathrm{O}$ & 2.81187100 & -0.33157000 & -2.36765500 \\
\hline C & 1.57319400 & -0.27070400 & -2.52754000 \\
\hline $\mathrm{O}$ & 0.65066100 & -0.24206400 & -1.65714700 \\
\hline $\mathrm{H}$ & 1.22071500 & -0.22930100 & -3.57566000 \\
\hline $\mathrm{H}$ & 3.51649100 & -0.56188600 & 3.48110400 \\
\hline C & -1.24933300 & 0.66601300 & 1.70727600 \\
\hline $\mathrm{H}$ & -0.64047900 & 1.56173500 & 1.85886700 \\
\hline C & -2.86702700 & 1.59977700 & 3.39313400 \\
\hline $\mathrm{H}$ & -2.35429800 & 1.18192400 & 4.26454900 \\
\hline $\mathrm{H}$ & -2.45832500 & 2.59966000 & 3.20580100 \\
\hline
\end{tabular}




$\begin{array}{lccc}\text { H } & -3.93348300 & 1.68804100 & 3.62323200 \\ \mathrm{C} & -1.69667900 & 2.48756700 & -0.72241300 \\ \mathrm{C} & -2.07650400 & 3.56088400 & 0.10693300 \\ \mathrm{C} & -0.65068600 & 2.66617800 & -1.64339200 \\ \mathrm{C} & -1.41510900 & 4.77054600 & 0.00613200 \\ \mathrm{H} & -2.88682400 & 3.43188300 & 0.81680300 \\ \mathrm{C} & 0.00473600 & 3.88366400 & -1.73806600 \\ \mathrm{H} & -0.34244100 & 1.82047800 & -2.25325900 \\ \mathrm{C} & -0.37203900 & 4.93432300 & -0.90910800 \\ \mathrm{H} & -1.71037600 & 5.59855100 & 0.64524100 \\ \mathrm{H} & 0.81907900 & 4.00609700 & -2.44761800 \\ \mathrm{H} & 0.14508700 & 5.88886100 & -0.97316100 \\ \mathrm{C} & -2.35230900 & 1.21019400 & -0.77361200 \\ \mathrm{H} & -2.05489600 & 0.53377700 & -1.57649700\end{array}$

TS(tx)-II (-1992.793784 a.u.)

$\begin{array}{lccc}\text { C } & -0.68040200 & -0.42745200 & 0.94327300 \\ \text { C } & -1.29603100 & -1.76783600 & 0.87296000 \\ \text { O } & -1.58623000 & -2.11506700 & -0.38788200 \\ \text { C } & -2.01728100 & -3.46384500 & -0.55216000 \\ \text { H } & -2.92403700 & -3.65391100 & 0.03215600 \\ \text { H } & -1.22608700 & -4.14738900 & -0.22935000 \\ \text { H } & -2.22184000 & -3.58562100 & -1.61671500 \\ \text { O } & -1.46607600 & -2.48111100 & 1.83777900 \\ \text { O } & -3.75611600 & 1.11863200 & 1.24011500 \\ \text { N } & -3.50161300 & 0.70358600 & -0.03453000 \\ \text { C } & -4.17924000 & -0.45999800 & -0.47394200 \\ \text { C } & -5.54262400 & -2.70560800 & -1.38639400 \\ \text { C } & -4.93312000 & -1.21924700 & 0.41945100 \\ \text { C } & -4.13579400 & -0.79505600 & -1.82896500 \\ \text { C } & -5.60806800 & -2.34212900 & -0.04510800 \\ \text { C } & -4.80558700 & -1.92546700 & -2.27426400 \\ \text { H } & -4.99813000 & -0.92241200 & 1.46096700 \\ \text { H } & -3.59390800 & -0.16647300 & -2.53144500 \\ \text { H } & -6.19197800 & -2.93562100 & 0.65382800 \\ \text { H } & -4.76638200 & -2.18621200 & -3.32896400 \\ \text { C } & -2.67908400 & 0.58144600 & 2.14168600 \\ \text { H } & -2.94479800 & -0.46359400 & 2.35363500 \\ \text { H } & -6.07514900 & -3.58362200 & -1.74213300 \\ \text { Rh } & 1.23428000 & -0.35486800 & 0.31957200 \\ \text { O } & 1.94560000 & -0.55876100 & 2.23286900 \\ \text { C } & 3.19903400 & -0.51498400 & 2.41342100 \\ \text { O } & 4.11363100 & -0.38163900 & 1.56662500 \\ \text { Rh } & 3.59544800 & -0.24489400 & -0.42902500\end{array}$




\begin{tabular}{lrrr} 
O & 3.43417800 & 1.81813500 & -0.24087100 \\
$\mathrm{C}$ & 2.36984800 & 2.29769700 & 0.21029900 \\
$\mathrm{O}$ & 1.30583400 & 1.69625300 & 0.55158100 \\
$\mathrm{H}$ & 2.33513600 & 3.39519700 & 0.32994500 \\
$\mathrm{O}$ & 3.52775000 & -2.31103000 & -0.53966100 \\
$\mathrm{C}$ & 2.44891700 & -2.89505800 & -0.28416500 \\
$\mathrm{O}$ & 1.33440000 & -2.39864400 & 0.05855900 \\
$\mathrm{H}$ & 2.45632400 & -3.99539000 & -0.36868800 \\
$\mathrm{O}$ & 2.86990600 & -0.12675900 & -2.36955400 \\
$\mathrm{C}$ & 1.63284100 & -0.08912000 & -2.54444800 \\
$\mathrm{O}$ & 0.70293500 & -0.12175800 & -1.67952500 \\
$\mathrm{H}$ & 1.28701000 & -0.01182100 & -3.59069900 \\
$\mathrm{H}$ & 3.51416300 & -0.60731500 & 3.46659200 \\
$\mathrm{C}$ & -1.35958600 & 0.65690800 & 1.44847300 \\
$\mathrm{H}$ & -0.78879000 & 1.57887700 & 1.58730300 \\
$\mathrm{C}$ & -2.76945900 & 1.44236800 & 3.37412200 \\
$\mathrm{H}$ & -2.08351700 & 1.06933900 & 4.14000500 \\
$\mathrm{H}$ & -2.49374200 & 2.47575800 & 3.13280700 \\
$\mathrm{H}$ & -3.78539000 & 1.43459300 & 3.78082900 \\
$\mathrm{C}$ & -1.89732600 & 2.51864200 & -0.58071500 \\
$\mathrm{C}$ & -2.59684800 & 3.56104600 & 0.04681600 \\
$\mathrm{C}$ & -0.77923900 & 2.81486100 & -1.37063800 \\
$\mathrm{C}$ & -2.17913700 & 4.87107800 & -0.12545400 \\
$\mathrm{H}$ & -3.46392700 & 3.33468000 & 0.66069900 \\
$\mathrm{C}$ & -0.36560000 & 4.12967800 & -1.53371200 \\
$\mathrm{H}$ & -0.23203600 & 1.99562700 & -1.83343800 \\
$\mathrm{C}$ & -1.06207500 & 5.15930800 & -0.90974600 \\
$\mathrm{H}$ & -2.72809600 & 5.67693300 & 0.35537300 \\
$\mathrm{H}$ & 0.50433500 & 4.34885400 & -2.14850300 \\
$\mathrm{H}$ & -0.73825600 & 6.18981600 & -1.03514100 \\
$\mathrm{C}$ & -2.30074700 & 1.11721000 & -0.50614700 \\
$\mathrm{H}$ & -1.85424700 & 0.45124300 & -1.25013100 \\
& & & \\
\hline & & & \\
\hline & &
\end{tabular}

(tx)-B (-1992.802711 a.u.)

$\begin{array}{lccc}\mathrm{C} & -0.36713400 & -0.56721800 & 0.90598600 \\ \mathrm{C} & -0.88123500 & -1.95169400 & 0.84811900 \\ \mathrm{O} & -1.45856000 & -2.24241700 & -0.30905600 \\ \mathrm{C} & -1.98518900 & -3.56879200 & -0.41458200 \\ \mathrm{H} & -2.69929600 & -3.75180100 & 0.39432800 \\ \mathrm{H} & -1.17075700 & -4.29748800 & -0.36510700 \\ \mathrm{H} & -2.49072400 & -3.60983600 & -1.37998400 \\ \mathrm{O} & -0.72579700 & -2.69066100 & 1.79441400 \\ \mathrm{O} & -3.19645900 & -0.72657700 & 1.83197500 \\ \mathrm{~N} & -3.50783700 & 0.04613300 & 0.67673100 \\ & & & \end{array}$




\begin{tabular}{|c|c|c|c|}
\hline C & -4.22731000 & -0.73385800 & -0.24528700 \\
\hline C & -5.71176400 & -2.22359100 & -2.09121500 \\
\hline C & -4.94431300 & -1.85932400 & 0.17479600 \\
\hline C & -4.27744900 & -0.34275700 & -1.58868300 \\
\hline C & -5.67497900 & -2.59435500 & -0.75035000 \\
\hline C & -5.01368000 & -1.09095400 & -2.49857600 \\
\hline $\mathrm{H}$ & -4.91553600 & -2.15176000 & 1.21917900 \\
\hline $\mathrm{H}$ & -3.75431100 & 0.55152500 & -1.92052000 \\
\hline $\mathrm{H}$ & -6.22270100 & -3.47169500 & -0.41266300 \\
\hline $\mathrm{H}$ & -5.03874400 & -0.77865000 & -3.54012200 \\
\hline C & -2.21116500 & 0.01026500 & 2.52857000 \\
\hline $\mathrm{H}$ & -1.72585300 & -0.72770500 & 3.17964600 \\
\hline $\mathrm{H}$ & -6.28543500 & -2.80450900 & -2.80868800 \\
\hline $\mathrm{Rh}$ & 1.44136900 & -0.26169500 & 0.26457000 \\
\hline $\mathrm{O}$ & 2.33501600 & -0.79104400 & 2.03390400 \\
\hline C & 3.59924800 & -0.73843200 & 2.12389000 \\
\hline $\mathrm{O}$ & 4.43248800 & -0.39948200 & 1.25274600 \\
\hline $\mathrm{Rh}$ & 3.72214100 & 0.12672400 & -0.60871700 \\
\hline $\mathrm{O}$ & 3.50538400 & 2.08735500 & 0.02820700 \\
\hline C & 2.45013600 & 2.41971100 & 0.61069200 \\
\hline $\mathrm{O}$ & 1.42878200 & 1.70910500 & 0.87196300 \\
\hline $\mathrm{H}$ & 2.37723100 & 3.46731300 & 0.94786900 \\
\hline $\mathrm{O}$ & 3.71846200 & -1.86806800 & -1.15691500 \\
\hline C & 2.69613000 & -2.55530500 & -0.93417600 \\
\hline $\mathrm{O}$ & 1.60352000 & -2.20303900 & -0.39254900 \\
\hline $\mathrm{H}$ & 2.73245900 & -3.61210900 & -1.24616600 \\
\hline $\mathrm{O}$ & 2.85938500 & 0.62000100 & -2.41990400 \\
\hline C & 1.61206200 & 0.60260800 & -2.50078200 \\
\hline $\mathrm{O}$ & 0.76363900 & 0.31266300 & -1.60043000 \\
\hline $\mathrm{H}$ & 1.17131000 & 0.87484800 & -3.47400900 \\
\hline $\mathrm{H}$ & 3.99969600 & -1.03297600 & 3.10755900 \\
\hline C & -1.27176100 & 0.47796600 & 1.40177800 \\
\hline $\mathrm{H}$ & -0.72731400 & 1.39699600 & 1.64210600 \\
\hline C & -2.77171700 & 1.16264500 & 3.33334500 \\
\hline $\mathrm{H}$ & -1.97362000 & 1.64034800 & 3.91164300 \\
\hline $\mathrm{H}$ & -3.22648500 & 1.92104300 & 2.68792000 \\
\hline $\mathrm{H}$ & -3.53148000 & 0.79333800 & 4.02837400 \\
\hline C & -2.47264700 & 2.18704700 & -0.08408800 \\
\hline C & -3.67386300 & 2.84930300 & 0.16188500 \\
\hline C & -1.40690700 & 2.88564900 & -0.65693100 \\
\hline C & -3.79877100 & 4.20283200 & -0.14107900 \\
\hline $\mathrm{H}$ & -4.50976700 & 2.29287300 & 0.58107400 \\
\hline C & -1.53412800 & 4.23596800 & -0.95392300 \\
\hline $\mathrm{H}$ & -0.47702800 & 2.35797300 & -0.86776100 \\
\hline
\end{tabular}




$\begin{array}{lrrr}\mathrm{C} & -2.73062900 & 4.90014900 & -0.69295700 \\ \mathrm{H} & -4.74031600 & 4.71132600 & 0.05349200 \\ \mathrm{H} & -0.69732000 & 4.77223900 & -1.39624500 \\ \mathrm{H} & -2.83077000 & 5.95733000 & -0.92746800 \\ \mathrm{C} & -2.28378300 & 0.71953800 & 0.22230700 \\ \mathrm{H} & -1.90809700 & 0.22505100 & -0.69220100\end{array}$

TS(tx)-III (-1992.784481 a.u.)

\begin{tabular}{|c|c|c|c|}
\hline C & -0.63217400 & -0.55813700 & 0.63660600 \\
\hline C & -1.07778600 & -1.97294500 & 0.47301200 \\
\hline 0 & -1.70743700 & -2.17673200 & -0.68519100 \\
\hline C & -2.21061500 & -3.49651300 & -0.87647900 \\
\hline $\mathrm{H}$ & -2.86749900 & -3.76944600 & -0.04347000 \\
\hline $\mathrm{H}$ & -1.38540800 & -4.21181400 & -0.94044000 \\
\hline $\mathrm{H}$ & -2.77406500 & -3.46719300 & -1.81011000 \\
\hline O & -0.81637400 & -2.81761500 & 1.29632900 \\
\hline O & -3.21405000 & -0.99113100 & 1.78757500 \\
\hline N & -3.38103200 & 0.01704700 & 0.79927200 \\
\hline C & -4.20762800 & -0.44502800 & -0.25246100 \\
\hline C & -5.90030600 & -1.32893400 & -2.28654600 \\
\hline C & -5.20003700 & -1.38719500 & 0.02635300 \\
\hline C & -4.06568900 & 0.06113200 & -1.54672400 \\
\hline C & -6.03446700 & -1.82562700 & -0.99396400 \\
\hline C & -4.91493600 & -0.38357400 & -2.55204600 \\
\hline $\mathrm{H}$ & -5.29988200 & -1.77583300 & 1.03418700 \\
\hline $\mathrm{H}$ & -3.29777200 & 0.79543600 & -1.77569600 \\
\hline $\mathrm{H}$ & -6.79962600 & -2.56536600 & -0.77007100 \\
\hline $\mathrm{H}$ & -4.79334300 & 0.01248800 & -3.55736500 \\
\hline C & -2.20731200 & -0.49899900 & 2.67930100 \\
\hline $\mathrm{H}$ & -1.75646700 & -1.40392300 & 3.10400400 \\
\hline $\mathrm{H}$ & -6.55693600 & -1.67560100 & -3.08022200 \\
\hline $\mathrm{Rh}$ & 1.33686200 & -0.22494200 & 0.07600400 \\
\hline $\mathrm{O}$ & 1.85291900 & -0.82135800 & 1.97481900 \\
\hline C & 3.08419500 & -0.86246800 & 2.26995300 \\
\hline 0 & 4.07303800 & -0.57472100 & 1.55439000 \\
\hline $\mathrm{Rh}$ & 3.73545500 & 0.05068100 & -0.38394200 \\
\hline $\mathrm{O}$ & 3.52384400 & 2.00051800 & 0.28324800 \\
\hline C & 2.40460700 & 2.37695900 & 0.70131600 \\
\hline O & 1.32006200 & 1.72206600 & 0.76293400 \\
\hline $\mathrm{H}$ & 2.34011600 & 3.41612100 & 1.06638600 \\
\hline O & 3.74128100 & -1.91551300 & -1.00999100 \\
\hline C & 2.65786300 & -2.54809200 & -0.99678700 \\
\hline 0 & 1.51235400 & -2.15072000 & -0.63289900 \\
\hline $\mathrm{H}$ & 2.69683400 & -3.59230800 & -1.34936600 \\
\hline
\end{tabular}




$\begin{array}{lrrr}\text { O } & 3.19797800 & 0.63934400 & -2.29384000 \\ \mathrm{C} & 1.98246600 & 0.67392700 & -2.59153700 \\ \mathrm{O} & 0.97979700 & 0.40283200 & -1.86229400 \\ \mathrm{H} & 1.73774700 & 0.98253400 & -3.62213400 \\ \mathrm{H} & 3.30302100 & -1.19554200 & 3.29821100 \\ \mathrm{C} & -1.25181000 & 0.21718400 & 1.74269400 \\ \mathrm{H} & -0.62192700 & 0.99687000 & 2.17808200 \\ \mathrm{C} & -2.77556400 & 0.41112800 & 3.74359800 \\ \mathrm{H} & -1.98316500 & 0.73376100 & 4.42752600 \\ \mathrm{H} & -3.22587300 & 1.30616000 & 3.29888500 \\ \mathrm{H} & -3.54242400 & -0.11126700 & 4.32230000 \\ \mathrm{C} & -2.14486400 & 2.07124400 & 0.12024700 \\ \mathrm{C} & -3.07131200 & 2.93989700 & 0.70019800 \\ \mathrm{C} & -1.25713600 & 2.53961600 & -0.84536700 \\ \mathrm{C} & -3.08872300 & 4.27891400 & 0.32980400 \\ \mathrm{H} & -3.78809200 & 2.55744600 & 1.42381300 \\ \mathrm{C} & -1.27924400 & 3.88219000 & -1.20716400 \\ \mathrm{H} & -0.55045500 & 1.85722500 & -1.31507700 \\ \mathrm{C} & -2.18927100 & 4.75433400 & -0.61996500 \\ \mathrm{H} & -3.81384100 & 4.95120800 & 0.78198700 \\ \mathrm{H} & -0.58319500 & 4.24354600 & -1.96038500 \\ \mathrm{H} & -2.20592300 & 5.80233500 & -0.90934700 \\ \mathrm{C} & -2.13099900 & 0.64039200 & 0.57708000 \\ \mathrm{H} & -1.46171300 & 0.06335800 & -0.32676400\end{array}$

(t)-C (-1992.839796 a.u.)

$\begin{array}{lccc}\text { C } & -0.58033400 & -0.11131400 & -0.35926700 \\ \mathrm{C} & -0.96383900 & -1.38804500 & -0.97667200 \\ \mathrm{O} & -0.84550900 & -1.32093900 & -2.32439600 \\ \mathrm{C} & -0.98491800 & -2.56546900 & -2.99100800 \\ \mathrm{H} & -1.98088200 & -2.99252200 & -2.82545100 \\ \mathrm{H} & -0.23757500 & -3.28014200 & -2.63139600 \\ \mathrm{H} & -0.83223900 & -2.36028800 & -4.05191400 \\ \mathrm{O} & -1.31900000 & -2.40522200 & -0.41060700 \\ \mathrm{O} & -2.76608700 & -1.35362200 & 1.76601400 \\ \mathrm{~N} & -3.38132500 & -0.40788500 & 0.97490000 \\ \mathrm{C} & -4.62620800 & -0.85493900 & 0.45064200 \\ \mathrm{C} & -7.02236300 & -1.73746400 & -0.57767800 \\ \mathrm{C} & -5.65111400 & -1.19011000 & 1.32779200 \\ \mathrm{C} & -4.76148700 & -0.97977900 & -0.92929500 \\ \mathrm{C} & -6.85836200 & -1.63220300 & 0.80129700 \\ \mathrm{C} & -5.97651000 & -1.41586900 & -1.43989400 \\ \mathrm{H} & -5.49043600 & -1.10280800 & 2.39890300 \\ \mathrm{H} & -3.92079800 & -0.74142300 & -1.57888600\end{array}$




\begin{tabular}{|c|c|c|c|}
\hline $\mathrm{H}$ & -7.67335400 & -1.89578400 & 1.46994300 \\
\hline $\mathrm{H}$ & -6.10176100 & -1.51872100 & -2.51432400 \\
\hline C & -1.40438000 & -0.86976700 & 2.01372200 \\
\hline $\mathrm{H}$ & -0.75399400 & -1.72281900 & 1.81175100 \\
\hline $\mathrm{H}$ & -7.96986200 & -2.08242600 & -0.98374400 \\
\hline $\mathrm{Rh}$ & 1.57091400 & -0.08842200 & -0.10187900 \\
\hline $\mathrm{O}$ & 1.54576600 & -0.84681700 & 1.82948000 \\
\hline C & 2.65319900 & -0.95495200 & 2.43591400 \\
\hline 0 & 3.80217400 & -0.65466100 & 2.03793000 \\
\hline $\mathrm{Rh}$ & 4.02168700 & 0.11939900 & 0.12704000 \\
\hline $\mathrm{O}$ & 3.66060300 & 2.03357000 & 0.85804300 \\
\hline C & 2.48181500 & 2.44193000 & 0.95120900 \\
\hline $\mathrm{O}$ & 1.40388600 & 1.83733100 & 0.66041300 \\
\hline $\mathrm{H}$ & 2.34967500 & 3.46713200 & 1.34049400 \\
\hline 0 & 4.14535500 & -1.80201700 & -0.62149600 \\
\hline C & 3.08121800 & -2.39128200 & -0.93318600 \\
\hline $\mathrm{O}$ & 1.88838800 & -1.97844800 & -0.85343000 \\
\hline $\mathrm{H}$ & 3.19305200 & -3.41372300 & -1.33372600 \\
\hline 0 & 4.02569200 & 0.86766200 & -1.80110400 \\
\hline C & 2.92751100 & 0.99424500 & -2.39624500 \\
\hline $\mathrm{O}$ & 1.76360100 & 0.71304700 & -1.98909300 \\
\hline $\mathrm{H}$ & 2.97485500 & 1.40958000 & -3.41805900 \\
\hline $\mathrm{H}$ & 2.58541500 & -1.36906300 & 3.45803200 \\
\hline C & -1.20611000 & 0.24848300 & 0.98832800 \\
\hline $\mathrm{H}$ & -0.65583500 & 1.08801600 & 1.42878100 \\
\hline C & -1.32556200 & -0.38416100 & 3.43951500 \\
\hline $\mathrm{H}$ & -0.29541100 & -0.08704500 & 3.66075300 \\
\hline $\mathrm{H}$ & -1.98116800 & 0.48289600 & 3.59687000 \\
\hline $\mathrm{H}$ & -1.62053400 & -1.17403800 & 4.13661900 \\
\hline C & -3.04429800 & 1.86248100 & 0.05717200 \\
\hline C & -4.39839000 & 2.20911500 & -0.08955600 \\
\hline C & -2.06031700 & 2.80960100 & -0.27918500 \\
\hline $\mathrm{C}$ & -4.75214300 & 3.45337000 & -0.58605200 \\
\hline $\mathrm{H}$ & -5.17998200 & 1.51260900 & 0.20034800 \\
\hline C & -2.42534800 & 4.04894700 & -0.78205700 \\
\hline $\mathrm{H}$ & -1.00474200 & 2.58053400 & -0.14579400 \\
\hline C & -3.76893200 & 4.37243500 & -0.94428000 \\
\hline $\mathrm{H}$ & -5.80338700 & 3.70867400 & -0.69077700 \\
\hline $\mathrm{H}$ & -1.65255300 & 4.76604900 & -1.04536000 \\
\hline $\mathrm{H}$ & -4.05148500 & 5.34481600 & -1.34015200 \\
\hline C & -2.61352000 & 0.60475800 & 0.64746800 \\
\hline $\mathrm{H}$ & -0.65136700 & 0.70258200 & -1.08562900 \\
\hline
\end{tabular}

TS(t)-IV (-1992.78241 a.u.) 


\begin{tabular}{|c|c|c|c|}
\hline C & 0.20795300 & -1.56165900 & 0.93804000 \\
\hline C & -0.48080900 & -2.86059100 & 0.82764800 \\
\hline $\mathrm{O}$ & -1.14095300 & -3.12989800 & 1.96345700 \\
\hline C & -1.98247200 & -4.27630800 & 1.90718700 \\
\hline $\mathrm{H}$ & -1.39976500 & -5.17801600 & 1.69514400 \\
\hline $\mathrm{H}$ & -2.73899600 & -4.14944100 & 1.12647700 \\
\hline $\mathrm{H}$ & -2.45498000 & -4.35042800 & 2.88731600 \\
\hline $\mathrm{O}$ & -0.47063500 & -3.59361600 & -0.14012900 \\
\hline $\mathrm{O}$ & 3.47441900 & -2.24775600 & -0.78042900 \\
\hline$N$ & 3.72813600 & -1.02878300 & -0.12984000 \\
\hline C & 4.60211000 & -0.16170700 & -0.83610900 \\
\hline C & 6.36834400 & 1.48423600 & -2.20414800 \\
\hline C & 4.26088600 & 1.17020100 & -1.06990100 \\
\hline C & 5.81224300 & -0.68014800 & -1.29552100 \\
\hline C & 5.15723900 & 1.99125500 & -1.74369400 \\
\hline C & 6.68698000 & 0.14564500 & -1.98735300 \\
\hline $\mathrm{H}$ & 3.29881500 & 1.55307500 & -0.73399600 \\
\hline $\mathrm{H}$ & 6.04315000 & -1.72621000 & -1.11349000 \\
\hline $\mathrm{H}$ & 4.89584800 & 3.03087400 & -1.92549700 \\
\hline $\mathrm{H}$ & 7.62834400 & -0.25709500 & -2.35290600 \\
\hline C & 2.03447400 & -2.35620700 & -0.89900100 \\
\hline $\mathrm{H}$ & 1.80077600 & -3.40833900 & -0.71594100 \\
\hline $\mathrm{H}$ & 7.06083500 & 2.12963600 & -2.73853500 \\
\hline $\mathrm{Rh}$ & -1.42966000 & -0.15384100 & 0.03151400 \\
\hline $\mathrm{O}$ & -1.50800800 & -0.99303200 & -1.84905200 \\
\hline C & -2.35066400 & -0.54030600 & -2.67704800 \\
\hline $\mathrm{O}$ & -3.16822800 & 0.40097300 & -2.52948100 \\
\hline $\mathrm{Rh}$ & -3.19564300 & 1.38519400 & -0.72027000 \\
\hline $\mathrm{O}$ & -1.68978600 & 2.64153400 & -1.38579100 \\
\hline C & -0.49903600 & 2.29584200 & -1.22390600 \\
\hline $\mathrm{O}$ & -0.04895200 & 1.24386900 & -0.67300600 \\
\hline $\mathrm{H}$ & 0.27181300 & 2.98862000 & -1.60196500 \\
\hline $\mathrm{O}$ & -4.56995400 & 0.02474200 & -0.01983200 \\
\hline C & -4.13710300 & -1.04745100 & 0.46941800 \\
\hline 0 & -2.93927400 & -1.41926200 & 0.62866300 \\
\hline $\mathrm{H}$ & -4.89867600 & -1.76871600 & 0.81127700 \\
\hline $\mathrm{O}$ & -3.10491900 & 2.24759900 & 1.15648100 \\
\hline C & -2.31484800 & 1.76403000 & 2.00116600 \\
\hline $\mathrm{O}$ & -1.50354900 & 0.80040300 & 1.86571800 \\
\hline $\mathrm{H}$ & -2.31645100 & 2.23641700 & 2.99856300 \\
\hline $\mathrm{H}$ & -2.36032800 & -1.04507600 & -3.65729600 \\
\hline C & 1.54931000 & -1.45600600 & 0.23486900 \\
\hline $\mathrm{H}$ & 0.61416700 & -0.72228200 & -0.12639400 \\
\hline C & 1.57409900 & -1.91182300 & -2.26711400 \\
\hline
\end{tabular}




$\begin{array}{lrrr}\mathrm{H} & 0.48720500 & -2.01841500 & -2.34749500 \\ \mathrm{H} & 1.83442300 & -0.85561000 & -2.42498000 \\ \mathrm{H} & 2.05464700 & -2.51292100 & -3.04506600 \\ \mathrm{C} & 2.77800800 & 0.33613400 & 1.65704300 \\ \mathrm{C} & 4.01303100 & 0.46481600 & 2.31346900 \\ \mathrm{C} & 1.70461300 & 1.14069000 & 2.05825400 \\ \mathrm{C} & 4.16259600 & 1.37384800 & 3.34859400 \\ \mathrm{H} & 4.84444600 & -0.16962500 & 2.01554900 \\ \mathrm{C} & 1.86374300 & 2.04884300 & 3.09673500 \\ \mathrm{H} & 0.75189200 & 1.08742800 & 1.54386500 \\ \mathrm{C} & 3.08842900 & 2.16875800 & 3.74449900 \\ \mathrm{H} & 5.12068000 & 1.45861900 & 3.85526700 \\ \mathrm{H} & 1.01886400 & 2.66517600 & 3.39592700 \\ \mathrm{H} & 3.20838500 & 2.87930900 & 4.55883300 \\ \mathrm{C} & 2.63816200 & -0.66335400 & 0.60387900 \\ \mathrm{H} & 0.21158700 & -1.09325700 & 1.92344900\end{array}$

TS(cd)-I (-1992.794906 a.u.)

$\begin{array}{lccc}\text { C } & -1.07826800 & -1.41832200 & 0.78613000 \\ \mathrm{C} & -1.31262700 & -2.75451000 & 0.19018600 \\ \mathrm{O} & -0.94859200 & -3.73173000 & 1.03051900 \\ \mathrm{C} & -0.94960000 & -5.03783200 & 0.46241700 \\ \mathrm{H} & -0.26766300 & -5.07265800 & -0.39350300 \\ \mathrm{H} & -1.95320100 & -5.31996700 & 0.12939700 \\ \mathrm{H} & -0.60716400 & -5.70957100 & 1.25018900 \\ \mathrm{O} & -1.75409200 & -2.93769300 & -0.92256100 \\ \mathrm{~N} & -2.81069100 & 1.49892100 & -0.11721800 \\ \mathrm{C} & -3.67869800 & 0.47978900 & -0.66159900 \\ \mathrm{C} & -5.39868200 & -1.45404500 & -1.61666800 \\ \mathrm{C} & -3.24093400 & -0.37348500 & -1.66741100 \\ \mathrm{C} & -4.94930800 & 0.36803900 & -0.10329800 \\ \mathrm{C} & -4.11630200 & -1.34372200 & -2.14168000 \\ \mathrm{C} & -5.81212000 & -0.60061500 & -0.59495000 \\ \mathrm{H} & -2.20759900 & -0.34415200 & -2.00984800 \\ \mathrm{H} & -5.23311900 & 1.03740200 & 0.70317900 \\ \mathrm{H} & -3.76519000 & -2.04654800 & -2.89159600 \\ \mathrm{H} & -6.81085800 & -0.69337400 & -0.17556000 \\ \mathrm{C} & -2.02194700 & 0.28235100 & 2.28559200 \\ \mathrm{H} & -1.08819800 & 0.84015200 & 2.21946300 \\ \mathrm{H} & -6.07239100 & -2.22241900 & -1.98784500 \\ \mathrm{Rh} & 0.77573100 & -0.72411500 & 0.25717100 \\ \mathrm{O} & 1.58435200 & -1.39423600 & 2.02120500 \\ \mathrm{C} & 2.83568700 & -1.28295700 & 2.18524600 \\ \mathrm{O} & 3.69484900 & -0.80095800 & 1.40980400 \\ & & & \\ & & 542 & \\ & & & \\ & & & \\ & & & \\ & & & \end{array}$




$\begin{array}{lrrr}\text { Rh } & 3.07516600 & -0.05162700 & -0.41538100 \\ \text { O } & 2.71020900 & 1.77180600 & 0.48032300 \\ \mathrm{C} & 1.60418900 & 1.94843100 & 1.03724900 \\ \mathrm{O} & 0.61141000 & 1.16068300 & 1.10488200 \\ \mathrm{H} & 1.45363800 & 2.92294700 & 1.53382200 \\ \mathrm{O} & 3.28467300 & -1.92571700 & -1.25489000 \\ \mathrm{C} & 2.32156000 & -2.72195300 & -1.16675400 \\ \mathrm{O} & 1.19058800 & -2.54540600 & -0.62671400 \\ \mathrm{H} & 2.46564700 & -3.71854300 & -1.61896200 \\ \mathrm{O} & 2.24079900 & 0.62596500 & -2.19257600 \\ \mathrm{C} & 1.00848300 & 0.49844800 & -2.36070000 \\ \mathrm{O} & 0.13382000 & 0.02864400 & -1.57133800 \\ \mathrm{H} & 0.61071200 & 0.84669800 & -3.33325900 \\ \mathrm{H} & 3.20753600 & -1.66710100 & 3.15054100 \\ \mathrm{C} & -2.08868600 & -0.93132800 & 1.58035200 \\ \mathrm{H} & -3.00024700 & -1.52844400 & 1.71555600 \\ \mathrm{C} & -2.91913300 & 0.53202400 & 3.43716500 \\ \mathrm{H} & -3.91391300 & 0.10796000 & 3.26076700 \\ \mathrm{H} & -2.49164700 & 0.03833500 & 4.32070900 \\ \mathrm{H} & -3.01084700 & 1.59846200 & 3.65758700 \\ \mathrm{C} & -1.08642000 & 3.22343400 & -0.49465000 \\ \mathrm{C} & -1.13314400 & 3.90434500 & 0.73491200 \\ \mathrm{C} & -0.14840200 & 3.62824800 & -1.46050100 \\ \mathrm{C} & -0.26080200 & 4.95714700 & 0.97234300 \\ \mathrm{H} & -1.85824600 & 3.59618700 & 1.47870100 \\ \mathrm{C} & 0.73714700 & 4.66281500 & -1.20472000 \\ \mathrm{H} & -0.11607600 & 3.11232300 & -2.41908200 \\ \mathrm{C} & 0.67910100 & 5.33403300 & 0.01433500 \\ \mathrm{H} & -0.30998600 & 5.48723700 & 1.92061500 \\ \mathrm{H} & 1.47070700 & 4.94605600 & -1.95438000 \\ \mathrm{H} & 1.36728500 & 6.15075900 & 0.21797900 \\ \mathrm{O} & -2.95670700 & 1.73988300 & 1.15452200 \\ \mathrm{H} & -1.96495900 & 2.14697200 & -0.87650700 \\ & & 1.84043400 & -1.91882700\end{array}$

(cd)-A (-1992.790767 a.u.)

$\begin{array}{llll}\text { C } & -0.75137700 & -1.02527500 & -0.16180700 \\ \mathrm{C} & -0.98515400 & -1.84771100 & -1.36107300 \\ \mathrm{O} & -0.91963300 & -3.16471600 & -1.13464900 \\ \mathrm{C} & -0.93346900 & -3.97813200 & -2.30212500 \\ \mathrm{H} & -0.06841700 & -3.74352000 & -2.93162700 \\ \mathrm{H} & -1.84733900 & -3.82070100 & -2.88348700 \\ \mathrm{H} & -0.87696700 & -5.00987500 & -1.95303700 \\ \mathrm{O} & -1.22128100 & -1.36137900 & -2.45310700 \\ & & \end{array}$




\begin{tabular}{|c|c|c|c|}
\hline$N$ & -3.65075300 & 0.88871300 & 0.37169800 \\
\hline C & -4.89891400 & 0.21325300 & 0.30581900 \\
\hline C & -7.31694700 & -1.13417300 & 0.13571900 \\
\hline C & -5.70105000 & 0.37584900 & -0.82508200 \\
\hline C & -5.31769500 & -0.58081600 & 1.37137200 \\
\hline C & -6.90133700 & -0.31590400 & -0.91070800 \\
\hline C & -6.52841500 & -1.25336800 & 1.27621100 \\
\hline $\mathrm{H}$ & -5.40406600 & 1.05717500 & -1.61872100 \\
\hline $\mathrm{H}$ & -4.69939700 & -0.65793700 & 2.25911200 \\
\hline $\mathrm{H}$ & -7.52450800 & -0.19580100 & -1.79285300 \\
\hline $\mathrm{H}$ & -6.85488700 & -1.87910100 & 2.10261000 \\
\hline C & -1.85967900 & 0.09374600 & 1.79765100 \\
\hline $\mathrm{H}$ & -1.00838500 & 0.78147400 & 1.76740200 \\
\hline $\mathrm{H}$ & -8.26256400 & -1.66546600 & 0.06848200 \\
\hline $\mathrm{Rh}$ & 1.20952800 & -0.42610000 & 0.04510800 \\
\hline 0 & 1.19534600 & -1.12496900 & 1.99375700 \\
\hline C & 2.28114700 & -1.08832700 & 2.64614700 \\
\hline $\mathrm{O}$ & 3.40610400 & -0.66941800 & 2.28814900 \\
\hline $\mathrm{Rh}$ & 3.62307000 & 0.08994200 & 0.37161300 \\
\hline 0 & 3.03688700 & 1.96044600 & 1.04649500 \\
\hline C & 1.81213200 & 2.20855100 & 1.10744000 \\
\hline $\mathrm{O}$ & 0.82920800 & 1.47486800 & 0.78988600 \\
\hline $\mathrm{H}$ & 1.53421000 & 3.20870500 & 1.48898200 \\
\hline $\mathrm{O}$ & 4.01710300 & -1.81239700 & -0.33089900 \\
\hline C & 3.05166300 & -2.52736300 & -0.68833000 \\
\hline $\mathrm{O}$ & 1.81333300 & -2.26614100 & -0.66900900 \\
\hline $\mathrm{H}$ & 3.30450300 & -3.52967100 & -1.07629500 \\
\hline $\mathrm{O}$ & 3.62338200 & 0.79626700 & -1.57495300 \\
\hline C & 2.55676600 & 0.73181700 & -2.23047600 \\
\hline $\mathrm{O}$ & 1.41910800 & 0.30763800 & -1.87441500 \\
\hline $\mathrm{H}$ & 2.60547000 & 1.09942400 & -3.27213500 \\
\hline $\mathrm{H}$ & 2.21388300 & -1.48266200 & 3.67584200 \\
\hline C & -1.78911300 & -0.87782500 & 0.68901900 \\
\hline $\mathrm{H}$ & -2.68131600 & -1.51067900 & 0.56707100 \\
\hline C & -2.05396800 & -0.49071300 & 3.17243900 \\
\hline $\mathrm{H}$ & -2.82100700 & -1.27466400 & 3.17497300 \\
\hline $\mathrm{H}$ & -1.10601000 & -0.94660700 & 3.47551200 \\
\hline $\mathrm{H}$ & -2.32533200 & 0.28134100 & 3.90042700 \\
\hline C & -1.84989900 & 2.08929400 & -0.80801800 \\
\hline C & -1.68895400 & 3.14690600 & 0.10719500 \\
\hline C & -1.06591600 & 2.03948400 & -1.97269000 \\
\hline C & -0.78918300 & 4.15752700 & -0.17302000 \\
\hline $\mathrm{H}$ & -2.28695800 & 3.16906800 & 1.01405700 \\
\hline C & -0.14469600 & 3.04315800 & -2.22422300 \\
\hline
\end{tabular}




$\begin{array}{cccc}\mathrm{H} & -1.16082200 & 1.18137600 & -2.63848100 \\ \mathrm{C} & -0.01482300 & 4.10521000 & -1.33488900 \\ \mathrm{H} & -0.67896800 & 4.99024300 & 0.51723300 \\ \mathrm{H} & 0.47434500 & 2.99466900 & -3.11589500 \\ \mathrm{H} & 0.70056200 & 4.89812100 & -1.54126500 \\ \mathrm{O} & -3.10135800 & 1.00272800 & 1.60551700 \\ \mathrm{C} & -2.89577300 & 1.11783700 & -0.69009700 \\ \mathrm{H} & -3.21532200 & 0.59964900 & -1.59525800\end{array}$

TS(cd)-II (-1992.784418 a.u.)

\begin{tabular}{|c|c|c|c|}
\hline C & -0.71841800 & -0.94777400 & -0.26486200 \\
\hline C & -0.92794800 & -1.79877400 & -1.45318400 \\
\hline $\mathrm{O}$ & -0.91062800 & -3.10227100 & -1.16358600 \\
\hline C & -0.89377800 & -3.97161300 & -2.29309700 \\
\hline $\mathrm{H}$ & 0.00221700 & -3.78426300 & -2.89390000 \\
\hline $\mathrm{H}$ & -1.77948300 & -3.82113700 & -2.91757800 \\
\hline $\mathrm{H}$ & -0.87607300 & -4.98551700 & -1.89265700 \\
\hline $\mathrm{O}$ & -1.09970600 & -1.34703400 & -2.56880900 \\
\hline$N$ & -3.70139100 & 0.94597400 & 0.41967200 \\
\hline C & -4.91349500 & 0.21745800 & 0.35219900 \\
\hline C & -7.29133900 & -1.20988000 & 0.16140700 \\
\hline C & -5.65595100 & 0.26028000 & -0.83078300 \\
\hline C & -5.37620000 & -0.50150500 & 1.45376000 \\
\hline C & -6.83316300 & -0.46895400 & -0.92438400 \\
\hline C & -6.56515400 & -1.21190500 & 1.34817200 \\
\hline $\mathrm{H}$ & -5.32648600 & 0.87960400 & -1.66189700 \\
\hline $\mathrm{H}$ & -4.80921300 & -0.48766600 & 2.37841600 \\
\hline $\mathrm{H}$ & -7.40511200 & -0.44092400 & -1.84827300 \\
\hline $\mathrm{H}$ & -6.92350400 & -1.77610200 & 2.20553700 \\
\hline C & -1.89444100 & 0.12084900 & 1.72711500 \\
\hline $\mathrm{H}$ & -1.03796400 & 0.80325000 & 1.76893900 \\
\hline $\mathrm{H}$ & -8.21885400 & -1.77127800 & 0.08672700 \\
\hline $\mathrm{Rh}$ & 1.22091500 & -0.41074500 & 0.02466600 \\
\hline 0 & 1.13722600 & -1.10713700 & 1.97209600 \\
\hline C & 2.19651300 & -1.06380400 & 2.66820400 \\
\hline $\mathrm{O}$ & 3.33260400 & -0.64619300 & 2.34952000 \\
\hline $\mathrm{Rh}$ & 3.61863800 & 0.08784200 & 0.43422300 \\
\hline $\mathrm{O}$ & 3.02613600 & 1.96991600 & 1.05995000 \\
\hline C & 1.80141700 & 2.22792500 & 1.07464000 \\
\hline $\mathrm{O}$ & 0.82663000 & 1.48973900 & 0.74122700 \\
\hline $\mathrm{H}$ & 1.51405000 & 3.23592700 & 1.42376000 \\
\hline $\mathrm{O}$ & 4.02456300 & -1.82438000 & -0.23370200 \\
\hline C & 3.07016200 & -2.53270700 & -0.62966400 \\
\hline $\mathrm{O}$ & 1.83362700 & -2.25965800 & -0.66212000 \\
\hline
\end{tabular}




$\begin{array}{lccc}\mathrm{H} & 3.32923000 & -3.53814500 & -1.00406600 \\ \mathrm{O} & 3.70822300 & 0.77403800 & -1.51243400 \\ \mathrm{C} & 2.66540100 & 0.71995400 & -2.20589500 \\ \mathrm{O} & 1.51146800 & 0.31033800 & -1.88515900 \\ \mathrm{H} & 2.75301400 & 1.07926700 & -3.24672200 \\ \mathrm{H} & 2.08779900 & -1.44994900 & 3.69689400 \\ \mathrm{C} & -1.83760900 & -0.69980600 & 0.48778600 \\ \mathrm{H} & -2.70531000 & -1.36222900 & 0.34100400 \\ \mathrm{C} & -2.02859000 & -0.67339000 & 3.00355900 \\ \mathrm{H} & -2.79748500 & -1.44996500 & 2.91140300 \\ \mathrm{H} & -1.07018000 & -1.15875300 & 3.21092600 \\ \mathrm{H} & -2.28334300 & -0.01728600 & 3.84290600 \\ \mathrm{C} & -1.85502300 & 2.03960800 & -0.79221900 \\ \mathrm{C} & -1.80300200 & 3.15713100 & 0.05560200 \\ \mathrm{C} & -1.03387500 & 1.98338400 & -1.92606600 \\ \mathrm{C} & -0.95663000 & 4.21014700 & -0.24831700 \\ \mathrm{H} & -2.43497600 & 3.19257100 & 0.93889600 \\ \mathrm{C} & -0.18091700 & 3.03805500 & -2.21374100 \\ \mathrm{H} & -1.04925300 & 1.09284100 & -2.55472100 \\ \mathrm{C} & -0.14354500 & 4.15196800 & -1.38097900 \\ \mathrm{H} & -0.92819900 & 5.08350900 & 0.39890100 \\ \mathrm{H} & 0.45840100 & 2.98699100 & -3.09136600 \\ \mathrm{H} & -5.52205400 & 4.98056400 & -1.61251300 \\ \mathrm{O} & -3.11507500 & 1.00448700 & 1.65742700 \\ \mathrm{C} & -2.81780300 & 0.97009300 & -0.60414900 \\ \mathrm{H} & -3.13168200 & 0.42233600 & -1.49424400\end{array}$

(cd)-B (-1992.795901 a.u.)

$\begin{array}{lccc}\text { C } & -0.67148600 & -0.80481000 & -0.36472000 \\ \mathrm{C} & -0.88665400 & -1.68568300 & -1.53799400 \\ \mathrm{O} & -1.04638700 & -2.95246000 & -1.16142100 \\ \mathrm{C} & -1.07982300 & -3.90472000 & -2.22825100 \\ \mathrm{H} & -0.14216100 & -3.86251300 & -2.79081200 \\ \mathrm{H} & -1.91562000 & -3.69925400 & -2.90277200 \\ \mathrm{H} & -1.19839800 & -4.87888700 & -1.75491900 \\ \mathrm{O} & -0.91840200 & -1.27533700 & -2.67625600 \\ \mathrm{~N} & -3.72149200 & 1.03631400 & 0.51154700 \\ \mathrm{C} & -4.88755600 & 0.23837000 & 0.39773600 \\ \mathrm{C} & -7.22954600 & -1.26123100 & 0.10236200 \\ \mathrm{C} & -5.57495700 & 0.23455800 & -0.82269800 \\ \mathrm{C} & -5.39884700 & -0.48245600 & 1.47872600 \\ \mathrm{C} & -6.72899600 & -0.52182300 & -0.96649600 \\ \mathrm{C} & -6.56366200 & -1.22581500 & 1.32207200 \\ \mathrm{H} & -5.21686500 & 0.84624500 & -1.64827000 \\ & & & \\ & & 54 & \end{array}$




\begin{tabular}{|c|c|c|c|}
\hline $\mathrm{H}$ & -4.88737100 & -0.43967400 & 2.43433200 \\
\hline $\mathrm{H}$ & -7.24918400 & -0.52073300 & -1.92158300 \\
\hline $\mathrm{H}$ & -6.95108900 & -1.78556000 & 2.17049300 \\
\hline C & -1.92117200 & 0.11247000 & 1.70633800 \\
\hline $\mathrm{H}$ & -1.06218500 & 0.77689000 & 1.86903400 \\
\hline $\mathrm{H}$ & -8.13787200 & -1.84698500 & -0.01256500 \\
\hline $\mathrm{Rh}$ & 1.21546000 & -0.40627500 & 0.01149700 \\
\hline 0 & 1.14781200 & -1.18748300 & 1.92282900 \\
\hline C & 2.19801900 & -1.15474200 & 2.63559800 \\
\hline $\mathrm{O}$ & 3.33130300 & -0.71913800 & 2.33714400 \\
\hline $\mathrm{Rh}$ & 3.61117100 & 0.04649800 & 0.44089700 \\
\hline $\mathrm{O}$ & 3.04901300 & 1.91989500 & 1.10026400 \\
\hline C & 1.82578400 & 2.18716700 & 1.13196800 \\
\hline 0 & 0.84564100 & 1.45380200 & 0.80018900 \\
\hline $\mathrm{H}$ & 1.54142500 & 3.18865900 & 1.49551300 \\
\hline $\mathrm{O}$ & 3.98512400 & -1.85680500 & -0.27527200 \\
\hline C & 3.02788300 & -2.53432200 & -0.71141800 \\
\hline $\mathrm{O}$ & 1.79464800 & -2.23629600 & -0.74950300 \\
\hline $\mathrm{H}$ & 3.27208900 & -3.52925200 & -1.11979300 \\
\hline $\mathrm{O}$ & 3.75209400 & 0.78634100 & -1.47215500 \\
\hline C & 2.70812700 & 0.79189400 & -2.16473600 \\
\hline $\mathrm{O}$ & 1.54724400 & 0.39345800 & -1.85292100 \\
\hline $\mathrm{H}$ & 2.79399900 & 1.19470200 & -3.18780000 \\
\hline $\mathrm{H}$ & 2.07740600 & -1.57028700 & 3.64990700 \\
\hline C & -1.91713600 & -0.39722500 & 0.27487600 \\
\hline $\mathrm{H}$ & -2.64181300 & -1.23142200 & 0.18807900 \\
\hline C & -1.98566000 & -0.96976700 & 2.75605000 \\
\hline $\mathrm{H}$ & -2.79395500 & -1.67820100 & 2.53794600 \\
\hline $\mathrm{H}$ & -1.03496500 & -1.51091700 & 2.78873100 \\
\hline H & -2.16910800 & -0.52767500 & 3.74097300 \\
\hline C & -1.82176400 & 2.03075600 & -0.71864400 \\
\hline C & -1.84276100 & 3.13789600 & 0.12984700 \\
\hline C & -1.01119900 & 2.04800400 & -1.85258600 \\
\hline C & -1.04804400 & 4.24372400 & -0.14941800 \\
\hline $\mathrm{H}$ & -2.48159200 & 3.12609200 & 1.00916300 \\
\hline C & -0.21349500 & 3.15343000 & -2.12556000 \\
\hline $\mathrm{H}$ & -0.98465200 & 1.18346100 & -2.51645900 \\
\hline C & -0.22809500 & 4.25385100 & -1.27505800 \\
\hline $\mathrm{H}$ & -1.07413700 & 5.10558700 & 0.51415400 \\
\hline $\mathrm{H}$ & 0.41824700 & 3.15167200 & -3.01149500 \\
\hline $\mathrm{H}$ & 0.39120400 & 5.12142400 & -1.49253700 \\
\hline 0 & -3.12327700 & 0.93118800 & 1.78128100 \\
\hline C & -2.66888000 & 0.81437200 & -0.45478100 \\
\hline $\mathrm{H}$ & -3.10035700 & 0.44170000 & -1.39062100 \\
\hline
\end{tabular}


TS(cd)-III (-1992.70185 a.u.)

\begin{tabular}{|c|c|c|c|}
\hline C & 0.36352100 & 1.22039300 & -0.61790700 \\
\hline C & 0.10030800 & 2.41478700 & -1.46874000 \\
\hline $\mathrm{O}$ & -0.17033800 & 3.47914800 & -0.69260300 \\
\hline C & -0.65079900 & 4.62200700 & -1.39680400 \\
\hline $\mathrm{H}$ & -1.55530200 & 4.36504200 & -1.95702600 \\
\hline $\mathrm{H}$ & 0.10535200 & 4.99203400 & -2.09588800 \\
\hline $\mathrm{H}$ & -0.87115400 & 5.37347900 & -0.63806200 \\
\hline 0 & 0.10203200 & 2.42772700 & -2.67600800 \\
\hline$N$ & 3.55931200 & 0.60861400 & 0.39665900 \\
\hline C & 4.88812600 & 0.27979700 & 0.13237100 \\
\hline C & 7.50801100 & -0.45256300 & -0.42374600 \\
\hline C & 5.22752400 & -0.14743500 & -1.15494300 \\
\hline C & 5.85245700 & 0.35934600 & 1.13882400 \\
\hline C & 6.53574300 & -0.52316800 & -1.41914000 \\
\hline C & 7.16036800 & -0.00573300 & 0.84729800 \\
\hline $\mathrm{H}$ & 4.46785000 & -0.17644800 & -1.93309600 \\
\hline $\mathrm{H}$ & 5.56701700 & 0.69892300 & 2.12888200 \\
\hline $\mathrm{H}$ & 6.80006600 & -0.86043700 & -2.41819200 \\
\hline $\mathrm{H}$ & 7.91439600 & 0.05551000 & 1.62827700 \\
\hline C & 1.86574900 & 1.30810200 & 1.81388200 \\
\hline $\mathrm{H}$ & 1.59908100 & 0.34340600 & 2.27074000 \\
\hline $\mathrm{H}$ & 8.53352000 & -0.73913100 & -0.64121600 \\
\hline $\mathrm{Rh}$ & -1.31311000 & 0.17806900 & -0.19889800 \\
\hline 0 & -1.42621700 & 1.31826300 & 1.52730500 \\
\hline C & -2.42155800 & 1.15252200 & 2.29613400 \\
\hline $\mathrm{O}$ & -3.39238900 & 0.37381200 & 2.16948900 \\
\hline $\mathrm{Rh}$ & -3.45822100 & -0.84597700 & 0.49804500 \\
\hline $\mathrm{O}$ & -2.34235200 & -2.27535300 & 1.49118700 \\
\hline C & -1.09597700 & -2.15041500 & 1.49807000 \\
\hline 0 & -0.38394000 & -1.26040900 & 0.94489200 \\
\hline $\mathrm{H}$ & -0.52511400 & -2.91109700 & 2.05896500 \\
\hline $\mathrm{O}$ & -4.41169700 & 0.65127100 & -0.55598000 \\
\hline C & -3.70606000 & 1.48882700 & -1.16464200 \\
\hline $\mathrm{O}$ & -2.44371900 & 1.57021000 & -1.22584600 \\
\hline $\mathrm{H}$ & -4.24612300 & 2.26846700 & -1.72844900 \\
\hline $\mathrm{O}$ & -3.35781300 & -1.98064100 & -1.22303100 \\
\hline C & -2.37481200 & -1.81933400 & -1.98376400 \\
\hline $\mathrm{O}$ & -1.38664300 & -1.03793600 & -1.85147100 \\
\hline $\mathrm{H}$ & -2.35425300 & -2.43764600 & -2.89753000 \\
\hline $\mathrm{H}$ & -2.41565600 & 1.78865200 & 3.19877300 \\
\hline C & 1.49204000 & 1.37433400 & 0.35629200 \\
\hline $\mathrm{H}$ & 1.99805700 & 2.31233100 & 0.05615000 \\
\hline
\end{tabular}




$\begin{array}{cccc}\mathrm{C} & 1.52056200 & 2.48611800 & 2.67513200 \\ \mathrm{H} & 1.79371200 & 3.41879700 & 2.16895800 \\ \mathrm{H} & 0.43822400 & 2.48472300 & 2.83823800 \\ \mathrm{H} & 2.03215700 & 2.44246600 & 3.64117200 \\ \mathrm{C} & 2.12560300 & -1.16403200 & -0.40768600 \\ \mathrm{C} & 2.58252900 & -2.04232800 & 0.58521200 \\ \mathrm{C} & 1.49382200 & -1.68259800 & -1.53519400 \\ \mathrm{C} & 2.35121600 & -3.40454200 & 0.47550500 \\ \mathrm{H} & 3.10707000 & -1.64652900 & 1.45386600 \\ \mathrm{C} & 1.25249500 & -3.04798600 & -1.63789700 \\ \mathrm{H} & 1.16966800 & -1.01477500 & -2.33143300 \\ \mathrm{C} & 1.67634500 & -3.91108500 & -0.63465900 \\ \mathrm{H} & 2.69906700 & -4.07544500 & 1.25759700 \\ \mathrm{H} & 0.74064400 & -3.43473600 & -2.51616000 \\ \mathrm{H} & 1.49847500 & -4.98036900 & -0.72295300 \\ \mathrm{O} & 3.32314600 & 1.34404800 & 1.57717300 \\ \mathrm{C} & 2.38265200 & 0.30000500 & -0.23093100 \\ \mathrm{H} & 1.63827100 & 0.71709700 & -1.39670200\end{array}$

TS(cx)-I (-1992.79454 a.u.)

$\begin{array}{lccc}\text { C } & -0.95648700 & -0.92547800 & 0.26100900 \\ \mathrm{C} & -1.43413300 & -1.85992700 & -0.77730900 \\ \mathrm{O} & -1.62108900 & -3.10648900 & -0.33189100 \\ \mathrm{C} & -1.99833100 & -4.04882800 & -1.33016800 \\ \mathrm{H} & -1.23874600 & -4.09343100 & -2.11708600 \\ \mathrm{H} & -2.95886700 & -3.77436000 & -1.78056700 \\ \mathrm{H} & -2.07868600 & -5.01076500 & -0.82306600 \\ \mathrm{O} & -1.66309700 & -1.50764100 & -1.91997500 \\ \mathrm{~N} & -2.56674000 & 1.85281700 & -0.03030700 \\ \mathrm{C} & -1.53118800 & 2.70780900 & -0.56066800 \\ \mathrm{C} & 0.41364800 & 4.42156000 & -1.51185200 \\ \mathrm{C} & -0.79849900 & 2.33882100 & -1.68250700 \\ \mathrm{C} & -1.29294800 & 3.90249500 & 0.11047600 \\ \mathrm{C} & 0.17594700 & 3.21049500 & -2.15401200 \\ \mathrm{C} & -0.31808800 & 4.76256700 & -0.37590300 \\ \mathrm{H} & -0.91288000 & 1.35612600 & -2.13593900 \\ \mathrm{H} & -1.87158500 & 4.13655200 & 0.99825300 \\ \mathrm{H} & 0.77162500 & 2.92200400 & -3.01654000 \\ \mathrm{H} & -0.12983200 & 5.70416900 & 0.13380100 \\ \mathrm{C} & -1.68485600 & 0.51288700 & 2.10870600 \\ \mathrm{H} & -0.73692900 & 1.04597300 & 2.02856500 \\ \mathrm{H} & 1.18207900 & 5.09314700 & -1.88648900 \\ \mathrm{Rh} & 1.03975300 & -0.51443000 & 0.14820200 \\ \mathrm{O} & 1.22619400 & -1.00048500 & 2.14730100 \\ & & & \\ & & 549 & \\ & & & \\ & & & \end{array}$




\begin{tabular}{|c|c|c|c|}
\hline C & 2.38317300 & -0.96142600 & 2.66334300 \\
\hline $\mathrm{O}$ & 3.47888800 & -0.67916600 & 2.12566900 \\
\hline $\mathrm{Rh}$ & 3.50880200 & -0.23704600 & 0.10336200 \\
\hline $\mathrm{O}$ & 3.19335500 & 1.76729300 & 0.52951600 \\
\hline C & 2.02209000 & 2.14591400 & 0.75460600 \\
\hline $\mathrm{O}$ & 0.94724700 & 1.47310300 & 0.72231500 \\
\hline $\mathrm{H}$ & 1.88719400 & 3.21124800 & 1.01540700 \\
\hline $\mathrm{O}$ & 3.61602000 & -2.25632200 & -0.33051200 \\
\hline C & 2.54788700 & -2.88745700 & -0.50448900 \\
\hline $\mathrm{O}$ & 1.35353400 & -2.47496700 & -0.41322600 \\
\hline $\mathrm{H}$ & 2.64707700 & -3.95286900 & -0.77521200 \\
\hline $\mathrm{O}$ & 3.33095600 & 0.17096900 & -1.91655900 \\
\hline C & 2.18171700 & 0.18631700 & -2.41564200 \\
\hline $\mathrm{O}$ & 1.06309100 & -0.01151500 & -1.85690000 \\
\hline $\mathrm{H}$ & 2.12366600 & 0.40541600 & -3.49735600 \\
\hline $\mathrm{H}$ & 2.41637500 & -1.21177600 & 3.73814600 \\
\hline C & -1.84827900 & -0.60096600 & 1.24712800 \\
\hline $\mathrm{H}$ & -2.78750500 & -1.15901100 & 1.33922900 \\
\hline C & -2.39680400 & 0.58117200 & 3.40987200 \\
\hline $\mathrm{H}$ & -3.37765500 & 0.09638100 & 3.35591300 \\
\hline $\mathrm{H}$ & -1.79195400 & 0.04656700 & 4.15366500 \\
\hline $\mathrm{H}$ & -2.51701100 & 1.61323400 & 3.75084000 \\
\hline C & -4.37288200 & 0.20922500 & -0.41078500 \\
\hline C & -5.14250100 & 0.40654300 & 0.74813300 \\
\hline C & -4.70260000 & -0.83682100 & -1.28842800 \\
\hline C & -6.20402100 & -0.44168500 & 1.02130600 \\
\hline $\mathrm{H}$ & -4.88958300 & 1.21924500 & 1.42050700 \\
\hline C & -5.75076100 & -1.69487300 & -0.99119300 \\
\hline $\mathrm{H}$ & -4.09177500 & -0.99506700 & -2.17568100 \\
\hline C & -6.50297200 & -1.49952800 & 0.16394800 \\
\hline $\mathrm{H}$ & -6.80099000 & -0.28221200 & 1.91587200 \\
\hline $\mathrm{H}$ & -5.98391300 & -2.51616200 & -1.66431000 \\
\hline $\mathrm{H}$ & -7.32670900 & -2.17033800 & 0.39615600 \\
\hline $\mathrm{O}$ & -2.73551900 & 1.88575700 & 1.27000700 \\
\hline C & -3.25555400 & 1.04269200 & -0.79332000 \\
\hline $\mathrm{H}$ & -2.96916500 & 1.04925000 & -1.84181100 \\
\hline
\end{tabular}

(cx)-A (-1992.799901 a.u.)

$\begin{array}{lccc}C & -0.99169800 & -0.91727300 & 0.36010300 \\ C & -1.44159200 & -2.02639000 & -0.50007100 \\ \text { O } & -1.53325600 & -3.20306500 & 0.13537700 \\ C & -1.84580800 & -4.30901300 & -0.70091800 \\ H & -1.08372900 & -4.42314800 & -1.47919700 \\ H & -2.82130200 & -4.17503600 & -1.18162800 \\ & & \end{array}$




\begin{tabular}{|c|c|c|c|}
\hline $\mathrm{H}$ & -1.86040700 & -5.18617000 & -0.05261700 \\
\hline $\mathrm{O}$ & -1.72909200 & -1.87870000 & -1.67504100 \\
\hline$N$ & -2.58566100 & 1.87610100 & -0.03783600 \\
\hline C & -1.55194400 & 2.76340000 & -0.49438400 \\
\hline C & 0.37948800 & 4.52626800 & -1.36820800 \\
\hline C & -0.76252900 & 2.41158500 & -1.58263200 \\
\hline C & -1.37404500 & 3.96156400 & 0.18959400 \\
\hline C & 0.20184200 & 3.31008600 & -2.02021900 \\
\hline C & -0.40122400 & 4.84504700 & -0.25858000 \\
\hline $\mathrm{H}$ & -0.82090700 & 1.41866800 & -2.02495500 \\
\hline $\mathrm{H}$ & -1.99407600 & 4.18672300 & 1.05207000 \\
\hline $\mathrm{H}$ & 0.84308500 & 3.03540800 & -2.85405000 \\
\hline $\mathrm{H}$ & -0.25464800 & 5.78884500 & 0.26010800 \\
\hline C & -1.78515500 & 0.83079800 & 1.96448000 \\
\hline $\mathrm{H}$ & -0.79982800 & 1.29260800 & 1.84068500 \\
\hline $\mathrm{H}$ & 1.14360300 & 5.21914000 & -1.71128700 \\
\hline $\mathrm{Rh}$ & 1.02538100 & -0.49900100 & 0.14159400 \\
\hline $\mathrm{O}$ & 1.27127600 & -0.94635300 & 2.14113900 \\
\hline C & 2.44591300 & -0.92273800 & 2.61403600 \\
\hline $\mathrm{O}$ & 3.52911300 & -0.67120700 & 2.03508400 \\
\hline $\mathrm{Rh}$ & 3.50104700 & -0.24881500 & 0.00696500 \\
\hline $\mathrm{O}$ & 3.23265500 & 1.76238200 & 0.43430600 \\
\hline C & 2.07487300 & 2.15856000 & 0.69519800 \\
\hline $\mathrm{O}$ & 0.98688600 & 1.50870100 & 0.68474700 \\
\hline $\mathrm{H}$ & 1.97109300 & 3.22424400 & 0.97151500 \\
\hline $\mathrm{O}$ & 3.57269400 & -2.27200500 & -0.41144700 \\
\hline C & 2.49230700 & -2.89627400 & -0.52782100 \\
\hline $\mathrm{O}$ & 1.30726100 & -2.47351200 & -0.39055400 \\
\hline $\mathrm{H}$ & 2.57233400 & -3.96673200 & -0.78633200 \\
\hline $\mathrm{O}$ & 3.24932700 & 0.14231600 & -2.01326100 \\
\hline C & 2.08493300 & 0.15985200 & -2.47478200 \\
\hline $\mathrm{O}$ & 0.98270100 & -0.02121400 & -1.87863400 \\
\hline $\mathrm{H}$ & 1.99524900 & 0.36663700 & -3.55745200 \\
\hline $\mathrm{H}$ & 2.51285300 & -1.15696300 & 3.69125000 \\
\hline C & -1.88807900 & -0.45388000 & 1.25517200 \\
\hline $\mathrm{H}$ & -2.83036800 & -0.99408800 & 1.41869700 \\
\hline C & -2.26676200 & 0.83222800 & 3.38778600 \\
\hline $\mathrm{H}$ & -3.25472600 & 0.36147100 & 3.46396200 \\
\hline $\mathrm{H}$ & -1.56226000 & 0.24923200 & 3.98950400 \\
\hline $\mathrm{H}$ & -2.32209300 & 1.84676800 & 3.79497800 \\
\hline C & -4.35185400 & 0.20142500 & -0.51427100 \\
\hline C & -5.18154400 & 0.33388000 & 0.61365500 \\
\hline C & -4.59973900 & -0.81982400 & -1.44652300 \\
\hline C & -6.22784900 & -0.55337700 & 0.79848000 \\
\hline
\end{tabular}




$\begin{array}{lrrc}\mathrm{H} & -4.99459100 & 1.12791100 & 1.32890000 \\ \mathrm{C} & -5.62971500 & -1.72239800 & -1.23194000 \\ \mathrm{H} & -3.93413800 & -0.93418100 & -2.29913500 \\ \mathrm{C} & -6.44578400 & -1.58783200 & -0.11271300 \\ \mathrm{H} & -6.87594100 & -0.44640500 & 1.66427900 \\ \mathrm{H} & -5.79732600 & -2.52882300 & -1.94063200 \\ \mathrm{H} & -7.25950700 & -2.28995800 & 0.05238200 \\ \mathrm{O} & -2.78572700 & 1.82376000 & 1.29794000 \\ \mathrm{C} & -3.24907100 & 1.07435600 & -0.82399300 \\ \mathrm{H} & -2.94244100 & 1.13372200 & -1.86729400\end{array}$

TS(cx)-II (-1992.790303 a.u.)

\begin{tabular}{lccc} 
C & -0.93192300 & -1.09491300 & 0.54268900 \\
$\mathrm{C}$ & -1.22863000 & -2.37685300 & -0.13719700 \\
$\mathrm{O}$ & -1.09049400 & -3.41109300 & 0.69853000 \\
$\mathrm{C}$ & -1.14745400 & -4.69463400 & 0.08016400 \\
$\mathrm{H}$ & -0.35643300 & -4.77943500 & -0.67163000 \\
$\mathrm{H}$ & -2.11736000 & -4.85317800 & -0.40085000 \\
$\mathrm{H}$ & -0.99434200 & -5.42044300 & 0.87913200 \\
$\mathrm{O}$ & -1.53456000 & -2.47491400 & -1.30684300 \\
$\mathrm{~N}$ & -2.49453600 & 1.74416200 & 0.13513900 \\
$\mathrm{C}$ & -1.55827300 & 2.73318200 & -0.25778500 \\
$\mathrm{C}$ & 0.21329100 & 4.74427300 & -1.01498700 \\
$\mathrm{C}$ & -1.06225900 & 2.76501500 & -1.56117300 \\
$\mathrm{C}$ & -1.20675800 & 3.72832900 & 0.65473700 \\
$\mathrm{C}$ & -0.16113500 & 3.75852500 & -1.92265900 \\
$\mathrm{C}$ & -0.32616600 & 4.72917900 & 0.26833700 \\
$\mathrm{H}$ & -1.35787400 & 2.01838100 & -2.29266900 \\
$\mathrm{H}$ & -1.62293700 & 3.70489800 & 1.65624900 \\
$\mathrm{H}$ & 0.24004000 & 3.76723900 & -2.93338300 \\
$\mathrm{H}$ & -0.05672100 & 5.50359700 & 0.98297400 \\
$\mathrm{C}$ & -1.91997700 & 0.68819200 & 2.09425800 \\
$\mathrm{H}$ & -0.90918800 & 1.11218200 & 2.10299400 \\
$\mathrm{H}$ & 0.91052800 & 5.52368700 & -1.31000000 \\
$\mathrm{Rh}$ & 1.00313000 & -0.54549700 & 0.19807200 \\
$\mathrm{O}$ & 1.58525300 & -1.12356700 & 2.08657700 \\
$\mathrm{C}$ & 2.81919700 & -1.06428300 & 2.37012200 \\
$\mathrm{O}$ & 3.77600600 & -0.68280000 & 1.65594800 \\
$\mathrm{Rh}$ & 3.38270100 & -0.02537400 & -0.26754000 \\
$\mathrm{O}$ & 3.02738900 & 1.87427800 & 0.45542300 \\
$\mathrm{C}$ & 1.87442300 & 2.14120400 & 0.86173700 \\
$\mathrm{O}$ & 0.84906500 & 1.39528200 & 0.89187300 \\
$\mathrm{H}$ & 1.70917600 & 3.16318900 & 1.24510600 \\
$\mathrm{O}$ & 3.57585400 & -1.95871500 & -0.96529500 \\
& & & \\
& 552 & \\
\hline
\end{tabular}




$\begin{array}{lccc}\text { C } & 2.57009700 & -2.70405700 & -0.93235200 \\ \text { O } & 1.40183500 & -2.44426000 & -0.51901200 \\ \text { H } & 2.70752500 & -3.73137100 & -1.31185500 \\ \text { O } & 2.76306800 & 0.58868700 & -2.14865000 \\ \text { C } & 1.54686300 & 0.50736700 & -2.43396900 \\ \text { O } & 0.58501000 & 0.09113900 & -1.72104900 \\ \text { H } & 1.25785200 & 0.84243900 & -3.44655300 \\ \text { H } & 3.07278800 & -1.39695800 & 3.39129800 \\ \text { C } & -1.97789900 & -0.53811500 & 1.24941200 \\ \text { H } & -2.86499000 & -1.15923100 & 1.43141700 \\ \text { C } & -2.48827200 & 0.49807500 & 3.47607000 \\ H & -3.50407000 & 0.08776500 & 3.41616500 \\ \text { H } & -1.86271600 & -0.19809000 & 4.04242200 \\ \text { H } & -2.52665400 & 1.44996800 & 4.01451400 \\ \text { C } & -4.05956200 & -0.05042200 & -0.54985000 \\ \text { C } & -5.13607400 & 0.36227000 & 0.25063600 \\ \text { C } & -4.25499900 & -1.05757600 & -1.50348000 \\ \text { C } & -6.38114700 & -0.22356600 & 0.08667900 \\ \text { H } & -4.98621900 & 1.13929600 & 0.99456400 \\ \text { C } & -5.50452600 & -1.64535600 & -1.65212500 \\ \text { H } & -3.41243800 & -1.38820400 & -2.10650400 \\ \text { C } & -6.56906700 & -1.23088200 & -0.85924900 \\ \text { H } & -7.21381000 & 0.10566000 & 0.70349300 \\ \text { H } & -5.64515000 & -2.42764600 & -2.39362800 \\ \text { H } & -7.54778900 & -1.69004200 & -0.97686400 \\ \text { O } & -2.81751400 & 1.71090800 & 1.46524500 \\ \mathrm{C} & -2.72921000 & 0.56296900 & -0.48427100 \\ \text { H } & -2.07045300 & 0.35926300 & -1.33275100\end{array}$

(cx)-B (-1992.803766 a.u.)

$\begin{array}{lccc}\text { C } & -0.57387400 & -1.33527200 & 0.59640700 \\ \text { C } & -0.58213500 & -2.80071200 & 0.41671300 \\ \text { O } & -0.28215100 & -3.42761800 & 1.55174400 \\ \text { C } & -0.10047300 & -4.84240700 & 1.43118900 \\ \text { H } & 0.71594400 & -5.04901000 & 0.73266500 \\ \text { H } & -1.01610200 & -5.31873300 & 1.07021100 \\ \text { H } & 0.14999800 & -5.19767600 & 2.43025000 \\ \text { O } & -0.83672700 & -3.31649800 & -0.64590400 \\ \text { N } & -3.02092900 & 1.28936000 & 0.73845900 \\ \text { C } & -2.54332200 & 2.55953900 & 0.36226400 \\ \text { C } & -1.67105200 & 5.08533100 & -0.46769400 \\ \text { C } & -2.54431600 & 2.89465500 & -0.99783600 \\ \text { C } & -2.14525000 & 3.50738400 & 1.30732600 \\ \text { C } & -2.10050100 & 4.14560100 & -1.40316400 \\ & & & \end{array}$




\begin{tabular}{|c|c|c|c|}
\hline C & -1.70779900 & 4.75781700 & 0.88516700 \\
\hline $\mathrm{H}$ & -2.90611700 & 2.17556900 & -1.73090400 \\
\hline $\mathrm{H}$ & -2.18030000 & 3.25408200 & 2.36220400 \\
\hline $\mathrm{H}$ & -2.09973400 & 4.39079600 & -2.46285700 \\
\hline $\mathrm{H}$ & -1.39085800 & 5.48559700 & 1.62920200 \\
\hline C & -1.66220000 & 0.27562900 & 2.22922900 \\
\hline $\mathrm{H}$ & -0.80483600 & 0.93173100 & 2.02362800 \\
\hline $\mathrm{H}$ & -1.32940500 & 6.06581900 & -0.78889500 \\
\hline $\mathrm{Rh}$ & 1.09065200 & -0.45319000 & 0.07993300 \\
\hline 0 & 1.69081300 & -0.15798200 & 2.03230300 \\
\hline C & 2.81604600 & 0.38972400 & 2.24845700 \\
\hline $\mathrm{O}$ & 3.65686100 & 0.80105100 & 1.41919700 \\
\hline $\mathrm{Rh}$ & 3.22509400 & 0.59700800 & -0.58904600 \\
\hline $\mathrm{O}$ & 2.22829900 & 2.40722400 & -0.56070900 \\
\hline C & 1.01437800 & 2.41547700 & -0.25708500 \\
\hline $\mathrm{O}$ & 0.27585200 & 1.42683500 & 0.05433800 \\
\hline $\mathrm{H}$ & 0.49593000 & 3.39014900 & -0.25129700 \\
\hline $\mathrm{O}$ & 4.03699900 & -1.30499700 & -0.57019400 \\
\hline C & 3.30214300 & -2.27353600 & -0.27412400 \\
\hline $\mathrm{O}$ & 2.07382300 & -2.27006900 & 0.04565000 \\
\hline $\mathrm{H}$ & 3.77110700 & -3.27153900 & -0.28626000 \\
\hline $\mathrm{O}$ & 2.65506600 & 0.32252100 & -2.55076600 \\
\hline C & 1.54190500 & -0.20660200 & -2.76739900 \\
\hline $\mathrm{O}$ & 0.68330300 & -0.61759900 & -1.92809900 \\
\hline $\mathrm{H}$ & 1.25075100 & -0.34094100 & -3.82187900 \\
\hline $\mathrm{H}$ & 3.06850100 & 0.51159700 & 3.31475500 \\
\hline C & -1.82182500 & -0.74556100 & 1.09306700 \\
\hline $\mathrm{H}$ & -2.53440700 & -1.51686300 & 1.43113600 \\
\hline C & -1.59324400 & -0.33254800 & 3.60172700 \\
\hline $\mathrm{H}$ & -2.46742600 & -0.96902100 & 3.78143300 \\
\hline $\mathrm{H}$ & -0.68511800 & -0.93844800 & 3.69474300 \\
\hline $\mathrm{H}$ & -1.56831400 & 0.44910500 & 4.36698800 \\
\hline C & -3.62786800 & -0.66730000 & -0.69162200 \\
\hline C & -4.92532500 & -0.68556700 & -0.18078300 \\
\hline C & -3.31261000 & -1.43135400 & -1.81468200 \\
\hline C & -5.90182500 & -1.46009100 & -0.79570100 \\
\hline $\mathrm{H}$ & -5.15844900 & -0.07739400 & 0.69155900 \\
\hline C & -4.29110500 & -2.20859500 & -2.42509500 \\
\hline $\mathrm{H}$ & -2.29314400 & -1.42668200 & -2.20145500 \\
\hline C & -5.58665000 & -2.22248000 & -1.91773500 \\
\hline $\mathrm{H}$ & -6.91492400 & -1.46668700 & -0.39974300 \\
\hline $\mathrm{H}$ & -4.03952500 & -2.80298000 & -3.30020100 \\
\hline $\mathrm{H}$ & -6.35299100 & -2.82675500 & -2.39796000 \\
\hline $\mathrm{O}$ & -2.88530500 & 1.00971700 & 2.10449700 \\
\hline
\end{tabular}




$\begin{array}{llll}\mathrm{C} & -2.54121300 & 0.11779100 & -0.01364200 \\ \mathrm{H} & -1.79866700 & 0.44871200 & -0.75280300\end{array}$

TS(cx)-III (-1992.77687 a.u.)

\begin{tabular}{|c|c|c|c|}
\hline C & -0.99916900 & -1.03279700 & 0.37716900 \\
\hline C & -1.27196400 & -2.29483500 & -0.37865500 \\
\hline $\mathrm{O}$ & -1.15510300 & -3.32767300 & 0.46580900 \\
\hline C & -1.10555300 & -4.60872800 & -0.16153200 \\
\hline $\mathrm{H}$ & -0.26051700 & -4.64906500 & -0.85519300 \\
\hline $\mathrm{H}$ & -2.03135200 & -4.80412600 & -0.71038700 \\
\hline $\mathrm{H}$ & -0.97595200 & -5.33208800 & 0.64353400 \\
\hline $\mathrm{O}$ & -1.47957300 & -2.38962800 & -1.56424900 \\
\hline$N$ & -2.42583800 & 1.42418400 & 0.82949600 \\
\hline C & -1.87716200 & 2.49568500 & 0.10510000 \\
\hline C & -0.76502400 & 4.62080500 & -1.32016500 \\
\hline C & -1.72018600 & 2.40042400 & -1.27869800 \\
\hline C & -1.51745800 & 3.66849800 & 0.77273400 \\
\hline C & -1.15630900 & 3.46009000 & -1.97867500 \\
\hline C & -0.95949000 & 4.71789100 & 0.05595000 \\
\hline $\mathrm{H}$ & -2.00546900 & 1.49777700 & -1.81369800 \\
\hline $\mathrm{H}$ & -1.65646900 & 3.73141900 & 1.84666100 \\
\hline $\mathrm{H}$ & -1.01934900 & 3.36747800 & -3.05381500 \\
\hline $\mathrm{H}$ & -0.67072700 & 5.62438900 & 0.58375500 \\
\hline C & -1.47177000 & 0.27897800 & 2.56215600 \\
\hline $\mathrm{H}$ & -0.40236800 & 0.49668600 & 2.46104600 \\
\hline $\mathrm{H}$ & -0.32092100 & 5.44392300 & -1.87346900 \\
\hline $\mathrm{Rh}$ & 1.01365700 & -0.44339800 & 0.08554200 \\
\hline $\mathrm{O}$ & 1.54367800 & -1.03572600 & 1.99174600 \\
\hline C & 2.76153700 & -0.91207000 & 2.32143700 \\
\hline $\mathrm{O}$ & 3.71892300 & -0.47312200 & 1.64149200 \\
\hline $\mathrm{Rh}$ & 3.36729200 & 0.13368300 & -0.30517900 \\
\hline 0 & 2.92593400 & 2.04328400 & 0.33674200 \\
\hline C & 1.75152000 & 2.28149000 & 0.70467700 \\
\hline $\mathrm{O}$ & 0.76908900 & 1.48405700 & 0.76642900 \\
\hline $\mathrm{H}$ & 1.52760600 & 3.31450000 & 1.02162600 \\
\hline $\mathrm{O}$ & 3.64974600 & -1.80402200 & -0.95056200 \\
\hline C & 2.65974500 & -2.57290900 & -0.96923900 \\
\hline 0 & 1.46630000 & -2.33464000 & -0.62235700 \\
\hline $\mathrm{H}$ & 2.84356200 & -3.59778800 & -1.33423900 \\
\hline 0 & 2.80537300 & 0.69310200 & -2.21323500 \\
\hline C & 1.59402900 & 0.60928200 & -2.52812500 \\
\hline $\mathrm{O}$ & 0.62255700 & 0.19046000 & -1.83241700 \\
\hline $\mathrm{H}$ & 1.32826400 & 0.94023400 & -3.54634800 \\
\hline $\mathrm{H}$ & 3.00029700 & -1.23642000 & 3.34856900 \\
\hline
\end{tabular}




$\begin{array}{lrrr}\mathrm{C} & -1.90384600 & -0.73711800 & 1.51035200 \\ \mathrm{H} & -2.51554100 & -1.56870500 & 1.87002900 \\ \mathrm{C} & -1.83424500 & -0.10951200 & 3.96986500 \\ \mathrm{H} & -2.90285600 & -0.34648100 & 4.04162600 \\ \mathrm{H} & -1.25342800 & -0.98805400 & 4.27014100 \\ \mathrm{H} & -1.61255500 & 0.70448000 & 4.66590200 \\ \mathrm{C} & -3.80286400 & -0.34652500 & -0.26044000 \\ \mathrm{C} & -4.93548000 & -0.40124800 & 0.55571200 \\ \mathrm{C} & -3.90713000 & -0.62356000 & -1.62039800 \\ \mathrm{C} & -6.16583500 & -0.74137200 & 0.01218300 \\ \mathrm{H} & -4.84103700 & -0.15762300 & 1.61423300 \\ \mathrm{C} & -5.14244400 & -0.96980500 & -2.15950400 \\ \mathrm{H} & -3.01874500 & -0.60535300 & -2.24876600 \\ \mathrm{C} & -6.26897700 & -1.02828600 & -1.34760600 \\ \mathrm{H} & -7.04628300 & -0.78173100 & 0.64875800 \\ \mathrm{H} & -5.22030100 & -1.19465300 & -3.22011700 \\ \mathrm{H} & -7.23259700 & -1.29770200 & -1.77341900 \\ \mathrm{O} & -2.23143600 & 1.45473900 & 2.21965100 \\ \mathrm{C} & -2.52770400 & 0.09288500 & 0.39171300 \\ \mathrm{H} & -1.56435900 & -0.08342600 & -0.43767400\end{array}$

(c)-C (-1992.837653 a.u.)

$\begin{array}{lrrr}\mathrm{C} & 0.18644300 & 1.91195800 & 0.75148400 \\ \mathrm{C} & 1.16720700 & 3.01142500 & 0.72494500 \\ \mathrm{O} & 1.30629500 & 3.54884500 & -0.51912800 \\ \mathrm{C} & 2.38959500 & 4.45594900 & -0.65410700 \\ \mathrm{H} & 3.33692400 & 3.94398700 & -0.45413800 \\ \mathrm{H} & 2.29455400 & 5.29587000 & 0.04180700 \\ \mathrm{H} & 2.36455300 & 4.81125900 & -1.68667800 \\ \mathrm{O} & 1.82245100 & 3.40153300 & 1.66575900 \\ \mathrm{~N} & -2.77442800 & 0.85911200 & -0.79675400 \\ \mathrm{C} & -3.81746100 & -0.06726700 & -1.08986700 \\ \mathrm{C} & -5.79375600 & -1.89706500 & -1.66581100 \\ \mathrm{C} & -4.98106900 & -0.06266700 & -0.32856600 \\ \mathrm{C} & -3.63256400 & -0.94497600 & -2.15318900 \\ \mathrm{C} & -5.96895400 & -0.99390600 & -0.62026700 \\ \mathrm{C} & -4.63254300 & -1.86775300 & -2.43339200 \\ \mathrm{H} & -5.10163700 & 0.65252300 & 0.48097300 \\ \mathrm{H} & -2.71828200 & -0.89267900 & -2.73713200 \\ \mathrm{H} & -6.88299800 & -1.00727900 & -0.03302900 \\ \mathrm{H} & -4.50225300 & -2.56606800 & -3.25558100 \\ \mathrm{C} & -1.14284800 & 2.31370500 & -1.47934200 \\ \mathrm{H} & -0.24960400 & 1.83205000 & -1.89432000 \\ \mathrm{H} & -6.57153400 & -2.62278300 & -1.88882600 \\ & & & \\ & & 556 & \\ & & & \\ & & & \\ & & & \end{array}$




\begin{tabular}{|c|c|c|c|}
\hline $\mathrm{Rh}$ & 1.19047200 & 0.11121500 & 0.10035300 \\
\hline $\mathrm{O}$ & -0.51301800 & -0.83797700 & -0.68454700 \\
\hline C & -0.39296200 & -2.03062300 & -1.10193500 \\
\hline $\mathrm{O}$ & 0.61816100 & -2.76038500 & -1.13857700 \\
\hline $\mathrm{Rh}$ & 2.40793900 & -2.00598800 & -0.39780800 \\
\hline $\mathrm{O}$ & 1.85254500 & -2.62460800 & 1.50586300 \\
\hline C & 1.18774900 & -1.84126400 & 2.22461700 \\
\hline $\mathrm{O}$ & 0.76999000 & -0.67518700 & 1.96367500 \\
\hline $\mathrm{H}$ & 0.91846800 & -2.21220200 & 3.23041400 \\
\hline $\mathrm{O}$ & 2.83490500 & -1.17838300 & -2.25280800 \\
\hline C & 2.38712200 & -0.04056400 & -2.52620600 \\
\hline $\mathrm{O}$ & 1.68301800 & 0.73612700 & -1.81416000 \\
\hline $\mathrm{H}$ & 2.63444800 & 0.34999300 & -3.52963700 \\
\hline $\mathrm{O}$ & 4.08454800 & -1.09473100 & 0.38398000 \\
\hline C & 3.98894700 & 0.09541000 & 0.77038900 \\
\hline $\mathrm{O}$ & 2.97679700 & 0.85371800 & 0.77641600 \\
\hline $\mathrm{H}$ & 4.91138800 & 0.55760100 & 1.16203100 \\
\hline $\mathrm{H}$ & -1.33117500 & -2.48077900 & -1.48681800 \\
\hline C & -1.14157500 & 2.21533400 & 0.05720000 \\
\hline $\mathrm{H}$ & -1.57578200 & 3.15188100 & 0.47194000 \\
\hline C & -1.37266500 & 3.69250700 & -2.03762200 \\
\hline $\mathrm{H}$ & -2.29601100 & 4.12346800 & -1.63141500 \\
\hline $\mathrm{H}$ & -0.52716800 & 4.32909300 & -1.76375200 \\
\hline $\mathrm{H}$ & -1.45298300 & 3.66374400 & -3.12831600 \\
\hline C & -2.41155400 & 0.50691000 & 1.59396100 \\
\hline C & -2.35468400 & 1.28623400 & 2.75587800 \\
\hline C & -2.59365800 & -0.87909900 & 1.69416800 \\
\hline C & -2.50511200 & 0.69061100 & 3.99964600 \\
\hline $\mathrm{H}$ & -2.18958000 & 2.35913800 & 2.68390700 \\
\hline C & -2.72538300 & -1.46837300 & 2.94120800 \\
\hline $\mathrm{H}$ & -2.57686800 & -1.49155000 & 0.79712700 \\
\hline C & -2.68481500 & -0.68614600 & 4.09309200 \\
\hline $\mathrm{H}$ & -2.46310100 & 1.30058000 & 4.89769500 \\
\hline $\mathrm{H}$ & -2.84281800 & -2.54623000 & 3.01573200 \\
\hline $\mathrm{H}$ & -2.78295900 & -1.15413800 & 5.06954600 \\
\hline $\mathrm{O}$ & -2.29682100 & 1.49501900 & -1.90693400 \\
\hline C & -2.15980700 & 1.14532800 & 0.31047800 \\
\hline $\mathrm{H}$ & 0.01202100 & 1.66775100 & 1.80432600 \\
\hline
\end{tabular}

TS(c)-IV (-1992.772377 a.u.)

$\begin{array}{lrrr}C & 0.20467800 & -1.32250400 & 1.51931300 \\ C & -0.57244900 & -2.54295100 & 1.80991400 \\ O & -0.28708600 & -3.55270400 & 0.95996800 \\ C & -1.12409500 & -4.69631600 & 1.09883500 \\ & & \end{array}$




\begin{tabular}{|c|c|c|c|}
\hline $\mathrm{H}$ & -2.16151700 & -4.42882000 & 0.87578300 \\
\hline $\mathrm{H}$ & -1.07168600 & -5.09948900 & 2.11446700 \\
\hline $\mathrm{H}$ & -0.75740800 & -5.42744200 & 0.37688200 \\
\hline $\mathrm{O}$ & -1.37266900 & -2.63418800 & 2.71115700 \\
\hline$N$ & 3.03330100 & -0.36420800 & -0.67794300 \\
\hline C & 4.41639800 & -0.13958100 & -0.84423100 \\
\hline C & 7.12838300 & 0.34515800 & -1.27654700 \\
\hline C & 5.20386100 & 0.38127100 & 0.18614500 \\
\hline C & 4.98810400 & -0.42803300 & -2.08680300 \\
\hline C & 6.55189300 & 0.62927400 & -0.04334000 \\
\hline C & 6.33971300 & -0.19134500 & -2.29090800 \\
\hline $\mathrm{H}$ & 4.76976800 & 0.58818500 & 1.16031400 \\
\hline $\mathrm{H}$ & 4.36618400 & -0.84389400 & -2.87321300 \\
\hline $\mathrm{H}$ & 7.15874800 & 1.03479100 & 0.76289400 \\
\hline $\mathrm{H}$ & 6.77922500 & -0.42439800 & -3.25795000 \\
\hline C & 1.80311600 & -2.27407900 & -0.44985600 \\
\hline $\mathrm{H}$ & 0.91726900 & -2.63030300 & -0.98557600 \\
\hline $\mathrm{H}$ & 8.18534600 & 0.53461700 & -1.44456000 \\
\hline $\mathrm{Rh}$ & -1.45420100 & -0.18441300 & 0.21761800 \\
\hline $\mathrm{O}$ & -0.04958900 & 0.18780300 & -1.24495400 \\
\hline C & -0.38046800 & 0.88044800 & -2.24830200 \\
\hline $\mathrm{O}$ & -1.49534200 & 1.40235300 & -2.49985200 \\
\hline $\mathrm{Rh}$ & -3.00670600 & 1.12665400 & -1.12230100 \\
\hline $\mathrm{O}$ & -2.35818400 & 2.78903700 & -0.08870100 \\
\hline C & -1.49216000 & 2.62945500 & 0.80913600 \\
\hline $\mathrm{O}$ & -0.92946300 & 1.56029800 & 1.17849400 \\
\hline $\mathrm{H}$ & -1.17776100 & 3.53885800 & 1.34852800 \\
\hline $\mathrm{O}$ & -3.53847400 & -0.62948500 & -2.05997700 \\
\hline C & -2.97506600 & -1.69349500 & -1.69876500 \\
\hline $\mathrm{O}$ & -2.08872300 & -1.85618500 & -0.81221900 \\
\hline $\mathrm{H}$ & -3.29254700 & -2.60993100 & -2.22400300 \\
\hline $\mathrm{O}$ & -4.42316200 & 0.78276000 & 0.32461800 \\
\hline C & -4.08727200 & 0.09624900 & 1.32648500 \\
\hline $\mathrm{O}$ & -2.97187800 & -0.43524800 & 1.57771300 \\
\hline $\mathrm{H}$ & -4.86953400 & -0.06411500 & 2.08641200 \\
\hline $\mathrm{H}$ & 0.41835900 & 1.04323100 & -2.99210400 \\
\hline C & 1.43582500 & -1.26421700 & 0.62311500 \\
\hline $\mathrm{H}$ & 1.39420300 & -1.65522400 & 1.86742000 \\
\hline C & 2.68202000 & -3.40370700 & 0.04928900 \\
\hline $\mathrm{H}$ & 3.59146100 & -2.98907900 & 0.50375100 \\
\hline $\mathrm{H}$ & 2.15155800 & -4.00341500 & 0.79592900 \\
\hline $\mathrm{H}$ & 2.97784300 & -4.05568500 & -0.77822600 \\
\hline C & 2.28049900 & 1.09813600 & 1.15815600 \\
\hline C & 2.20825800 & 1.16511500 & 2.55269200 \\
\hline
\end{tabular}




$\begin{array}{lrrr}\mathrm{C} & 2.32282500 & 2.28304000 & 0.41314900 \\ \mathrm{C} & 2.15106600 & 2.39716300 & 3.19103000 \\ \mathrm{H} & 2.21219200 & 0.25026700 & 3.14381600 \\ \mathrm{C} & 2.26487500 & 3.51101700 & 1.05516400 \\ \mathrm{H} & 2.37950200 & 2.22146800 & -0.67158600 \\ \mathrm{C} & 2.17375600 & 3.57153900 & 2.44419700 \\ \mathrm{H} & 2.09376400 & 2.44006200 & 4.27569400 \\ \mathrm{H} & 2.28803600 & 4.42680200 & 0.46943000 \\ \mathrm{H} & 2.12828600 & 4.53533800 & 2.94553100 \\ \mathrm{O} & 2.53867900 & -1.46760000 & -1.39317100 \\ \mathrm{C} & 2.26961800 & -0.18043200 & 0.45059400 \\ \mathrm{H} & 0.07323800 & -0.51518000 & 2.24130900\end{array}$

TS(td)-V (-1992.779162 a.u.)

$\begin{array}{lccc}\mathrm{C} & 0.68427000 & 0.06675900 & 1.20331500 \\ \mathrm{C} & 0.52545700 & -0.26694200 & 2.64457400 \\ \mathrm{O} & 0.35857400 & 0.86366800 & 3.33674800 \\ \mathrm{C} & 0.01065500 & 0.67972200 & 4.70963700 \\ \mathrm{H} & 0.81350000 & 0.16168700 & 5.24414600 \\ \mathrm{H} & -0.90771500 & 0.09038400 & 4.78623800 \\ \mathrm{H} & -0.13773200 & 1.67904600 & 5.11798000 \\ \mathrm{O} & 0.51980400 & -1.38336500 & 3.10239300 \\ \mathrm{O} & 4.28581100 & 0.26018100 & 0.42993600 \\ \mathrm{~N} & 3.64257200 & -0.07653100 & -0.79646600 \\ \mathrm{C} & 3.58016900 & -1.49638900 & -0.95646600 \\ \mathrm{C} & 3.58302600 & -4.27630900 & -1.30455600 \\ \mathrm{C} & 4.67209400 & -2.26215100 & -0.53286300 \\ \mathrm{C} & 2.49583400 & -2.13199800 & -1.57038000 \\ \mathrm{C} & 4.66603300 & -3.63809600 & -0.70332100 \\ \mathrm{C} & 2.50631900 & -3.51500100 & -1.73682600 \\ \mathrm{H} & 5.51131100 & -1.76176100 & -0.05926700 \\ \mathrm{H} & 1.62907700 & -1.57483600 & -1.91852500 \\ \mathrm{H} & 5.52039400 & -4.21858600 & -0.36269400 \\ \mathrm{H} & 1.64853800 & -3.99281000 & -2.20494600 \\ \mathrm{C} & 3.29487300 & 0.29592400 & 1.47890700 \\ \mathrm{H} & 3.36956100 & 1.28910600 & 1.95365000 \\ \mathrm{H} & 3.58140100 & -5.35550300 & -1.43269800 \\ \mathrm{Rh} & -1.16410300 & -0.03029800 & 0.19303300 \\ \mathrm{O} & -0.85489700 & -2.03376300 & -0.16461100 \\ \mathrm{C} & -1.77141700 & -2.67569600 & -0.76031900 \\ \mathrm{O} & -2.87864700 & -2.25724800 & -1.17299800 \\ \mathrm{Rh} & -3.37087400 & -0.27175300 & -0.86174500 \\ \mathrm{O} & -2.48827800 & 0.23196400 & -2.65639600 \\ \mathrm{C} & -1.25228600 & 0.42351600 & -2.67587000 \\ & & & \\ & & & \\ & & & \end{array}$




$\begin{array}{lrrr}\mathrm{O} & -0.43085200 & 0.36946700 & -1.70968300 \\ \mathrm{H} & -0.80681400 & 0.67121700 & -3.65366700 \\ \mathrm{O} & -4.11079200 & -0.75539600 & 0.99814300 \\ \mathrm{C} & -3.32619100 & -0.72139900 & 1.97500300 \\ \mathrm{O} & -2.09115800 & -0.44200500 & 1.98650000 \\ \mathrm{H} & -3.75735300 & -0.96369200 & 2.96052000 \\ \mathrm{O} & -3.67760500 & 1.72915300 & -0.45848800 \\ \mathrm{C} & -2.75399500 & 2.37443400 & 0.09184900 \\ \mathrm{O} & -1.61529400 & 1.96434000 & 0.46688000 \\ \mathrm{H} & -2.94793400 & 3.44390200 & 0.27891000 \\ \mathrm{H} & -1.55188700 & -3.74252300 & -0.93418600 \\ \mathrm{C} & 1.97551400 & 0.27767100 & 0.72941300 \\ \mathrm{H} & 1.39261200 & -0.90475800 & 0.66046700 \\ \mathrm{C} & 3.55596300 & -0.79675700 & 2.49461500 \\ \mathrm{H} & 2.96626500 & -0.65318200 & 3.40520900 \\ \mathrm{H} & 3.33021300 & -1.78848900 & 2.08792700 \\ \mathrm{H} & 4.61549900 & -0.76653600 & 2.76508100 \\ \mathrm{C} & 2.51574300 & 2.12303900 & -0.83809200 \\ \mathrm{C} & 3.67334000 & 2.67689300 & -1.38157600 \\ \mathrm{C} & 1.46464000 & 2.95604300 & -0.45087300 \\ \mathrm{C} & 3.77368200 & 4.05372200 & -1.54642900 \\ \mathrm{H} & 4.49014700 & 2.02007500 & -1.66885400 \\ \mathrm{C} & 1.57291300 & 4.33308500 & -0.61535300 \\ \mathrm{H} & 0.55155000 & 2.53782800 & -0.02892300 \\ \mathrm{C} & 2.72579900 & 4.88540000 & -1.16336100 \\ \mathrm{H} & 4.67963000 & 4.47861600 & -1.97281400 \\ \mathrm{H} & 0.74792600 & 4.97375400 & -0.31204200 \\ \mathrm{H} & 2.80917000 & 5.96242200 & -1.29016700 \\ \mathrm{C} & 2.36939300 & 0.61984900 & -0.69134200 \\ \mathrm{H} & 1.64435300 & 0.25475400 & -1.41957000\end{array}$

(td)-D (-1015.139125 a.u.)

$\begin{array}{lccc}\text { C } & -2.16880900 & -0.36030900 & -0.47406900 \\ \mathrm{C} & -3.24992000 & -1.32704200 & -0.24651400 \\ \mathrm{O} & -4.41253400 & -0.86106900 & -0.73616100 \\ \mathrm{C} & -5.52708300 & -1.72831800 & -0.56138200 \\ \mathrm{H} & -5.71415100 & -1.91024300 & 0.50140200 \\ \mathrm{H} & -5.34997900 & -2.69145900 & -1.04994800 \\ \mathrm{H} & -6.37931000 & -1.22201200 & -1.01559800 \\ \mathrm{O} & -3.14547500 & -2.40558800 & 0.30124500 \\ \mathrm{O} & 0.67139400 & -1.06389200 & 1.53465200 \\ \mathrm{~N} & 1.25863800 & -0.10612000 & 0.67804800 \\ \mathrm{C} & 2.51691800 & -0.46751700 & 0.17299400 \\ \mathrm{C} & 5.07077200 & -1.07667500 & -0.80274700 \\ & & & \end{array}$




$\begin{array}{lccc}\text { C } & 3.23899200 & -1.53793300 & 0.71042100 \\ \mathrm{C} & 3.09601700 & 0.31640600 & -0.83398000 \\ \mathrm{C} & 4.50105400 & -1.83524400 & 0.21522200 \\ \mathrm{C} & 4.36028000 & 0.00281600 & -1.31595500 \\ \mathrm{H} & 2.79753400 & -2.12485000 & 1.50885800 \\ \mathrm{H} & 2.56943800 & 1.18937900 & -1.21536700 \\ \mathrm{H} & 5.04674800 & -2.67720400 & 0.63579000 \\ \mathrm{H} & 4.79440300 & 0.61944800 & -2.09991600 \\ \mathrm{C} & -0.39368600 & -1.69001200 & 0.80493900 \\ \mathrm{H} & -1.11359300 & -2.03567700 & 1.55299900 \\ \mathrm{H} & 6.05993600 & -1.31689200 & -1.18324900 \\ \mathrm{C} & -0.93235800 & -0.55359600 & -0.00898400 \\ \mathrm{H} & -2.41685700 & 0.54468400 & -1.02582300 \\ \mathrm{C} & 0.07587600 & -2.84495500 & -0.06295900 \\ \mathrm{H} & -0.79011300 & -3.31011600 & -0.54381200 \\ \mathrm{H} & 0.77443600 & -2.50612600 & -0.83712400 \\ \mathrm{H} & 0.58336600 & -3.59478300 & 0.55181600 \\ \mathrm{C} & -0.11536900 & 1.84137200 & -0.02196200 \\ \mathrm{C} & -0.26668600 & 2.33496200 & 1.27364300 \\ \mathrm{C} & -0.32229600 & 2.68432200 & -1.10973000 \\ \mathrm{C} & -0.62322100 & 3.66076800 & 1.47381900 \\ \mathrm{H} & -0.09103900 & 1.66662300 & 2.11506700 \\ \mathrm{C} & -0.68348700 & 4.01403100 & -0.90871400 \\ \mathrm{H} & -0.19238800 & 2.29705900 & -2.12103300 \\ \mathrm{C} & -0.83433900 & 4.50245800 & 0.38306100 \\ \mathrm{H} & -0.73676500 & 4.04326600 & 2.48543200 \\ \mathrm{H} & -0.83901400 & 4.66862400 & -1.76296200 \\ \mathrm{H} & -1.11182300 & 5.54153700 & 0.54335300 \\ \mathrm{C} & 0.22705700 & 0.38983800 & -0.23807200 \\ \mathrm{H} & 0.57335800 & 0.25373800 & -1.27830400\end{array}$

TS(td)-VI-(f) (-1015.01226 a.u.)

$\begin{array}{lccc}\text { C } & -2.03860900 & -0.55946600 & -0.79014400 \\ \mathrm{C} & -3.46275800 & -0.57738200 & -0.40451200 \\ \mathrm{O} & -4.28023400 & -0.47712500 & -1.46567900 \\ \mathrm{C} & -5.66877900 & -0.47419600 & -1.15397100 \\ \mathrm{H} & -5.91011300 & 0.34165100 & -0.46566100 \\ \mathrm{H} & -5.96350600 & -1.41853500 & -0.68559800 \\ \mathrm{H} & -6.19348200 & -0.34155400 & -2.10071700 \\ \mathrm{O} & -3.85165500 & -0.67510200 & 0.74104900 \\ \mathrm{O} & 0.64899500 & -1.96468200 & 0.86179200 \\ \mathrm{~N} & 1.16828700 & -0.68929000 & 0.48246400 \\ \mathrm{C} & 2.44426100 & -0.79402600 & -0.08849200 \\ \mathrm{C} & 5.03297300 & -0.94642800 & -1.12612100 \\ & & & \end{array}$




$\begin{array}{lccc}\mathrm{C} & 3.30152700 & -1.82085200 & 0.31643700 \\ \mathrm{C} & 2.88310900 & 0.15343600 & -1.01855800 \\ \mathrm{C} & 4.58293100 & -1.89353100 & -0.21185200 \\ \mathrm{C} & 4.17552400 & 0.07563100 & -1.51978300 \\ \mathrm{H} & 2.95002000 & -2.55294100 & 1.03581500 \\ \mathrm{H} & 2.21658900 & 0.94743700 & -1.34514800 \\ \mathrm{H} & 5.23966800 & -2.70145700 & 0.10293300 \\ \mathrm{H} & 4.50786100 & 0.81971000 & -2.24013900 \\ \mathrm{C} & -0.71195400 & -1.72730000 & 1.27497900 \\ \mathrm{H} & -0.71400400 & -1.26827000 & 2.28206300 \\ \mathrm{H} & 6.03995600 & -1.00605600 & -1.53064100 \\ \mathrm{C} & -1.06922100 & -0.72382500 & 0.22775700 \\ \mathrm{C} & -1.45273700 & -3.03485500 & 1.26074300 \\ \mathrm{H} & -2.49689600 & -2.85529900 & 1.53427600 \\ \mathrm{H} & -1.41855100 & -3.47343700 & 0.25777000 \\ \mathrm{H} & -1.01383300 & -3.74227300 & 1.96994700 \\ \mathrm{C} & 0.13988000 & 1.53281900 & 0.17150500 \\ \mathrm{C} & 0.50217700 & 1.96901900 & 1.45081800 \\ \mathrm{C} & -0.13644300 & 2.48147000 & -0.81107000 \\ \mathrm{C} & 0.55723800 & 3.32488000 & 1.74077100 \\ \mathrm{H} & 0.74331000 & 1.23055300 & 2.21212700 \\ \mathrm{C} & -0.08965300 & 3.84042500 & -0.51676600 \\ \mathrm{H} & -0.38203300 & 2.15278700 & -1.81990100 \\ \mathrm{C} & 0.25675000 & 4.26557700 & 0.75936600 \\ \mathrm{H} & 0.83212500 & 3.64919200 & 2.74166200 \\ \mathrm{H} & -0.31133100 & 4.56673200 & -1.29487900 \\ \mathrm{H} & 0.30232000 & 5.32753000 & 0.98827500 \\ \mathrm{C} & 0.07493100 & 0.04505400 & -0.07466800 \\ \mathrm{H} & -0.82925100 & 0.22185400 & -1.24691600 \\ \mathrm{H} & -1.84058200 & -0.95602600 & -1.78845000\end{array}$

TS(td)-VII-(f) (-1015.064703 a.u.)

$\begin{array}{cccc}\text { C } & 2.51784200 & 0.33613200 & -0.06577200 \\ \mathrm{C} & 3.70103900 & -0.44066300 & 0.06231100 \\ \mathrm{O} & 4.81578800 & 0.35077300 & 0.04435600 \\ \mathrm{C} & 6.03772000 & -0.35213100 & 0.14701800 \\ \mathrm{H} & 6.16760200 & -1.05444600 & -0.68420300 \\ \mathrm{H} & 6.09406300 & -0.92440900 & 1.08002500 \\ \mathrm{H} & 6.82775400 & 0.40176200 & 0.12369500 \\ \mathrm{O} & 3.79304600 & -1.66201700 & 0.17530200 \\ \mathrm{O} & -0.16211300 & -1.78357700 & -1.19448500 \\ \mathrm{~N} & -0.91642600 & -0.67911700 & -0.75651400 \\ \mathrm{C} & -2.18239200 & -1.02361600 & -0.21383900 \\ \mathrm{C} & -4.67873600 & -1.72582600 & 0.78427600\end{array}$




$\begin{array}{lccc}\mathrm{C} & -2.93597900 & -2.00187700 & -0.86265300 \\ \mathrm{C} & -2.66607100 & -0.40195500 & 0.93719700 \\ \mathrm{C} & -4.17827900 & -2.35177000 & -0.35467000 \\ \mathrm{C} & -3.91932200 & -0.75329600 & 1.42443700 \\ \mathrm{H} & -2.52987800 & -2.48412800 & -1.74656700 \\ \mathrm{H} & -2.06625300 & 0.34565800 & 1.45053700 \\ \mathrm{H} & -4.76255400 & -3.11923900 & -0.85603300 \\ \mathrm{H} & -4.29528300 & -0.26909600 & 2.32220700 \\ \mathrm{C} & 1.03885800 & -1.77060200 & -0.38172700 \\ \mathrm{H} & 1.84703800 & -2.12129600 & -1.02617400 \\ \mathrm{H} & -5.65545100 & -2.00013100 & 1.17396700 \\ \mathrm{C} & 1.23838700 & -0.28125600 & -0.06042000 \\ \mathrm{H} & 2.60866500 & 1.41725300 & -0.06395600 \\ \mathrm{C} & 0.88281300 & -2.64723300 & 0.83614400 \\ \mathrm{H} & 1.81583100 & -2.62372800 & 1.40784000 \\ \mathrm{H} & 0.05562000 & -2.29500700 & 1.46817500 \\ \mathrm{H} & 0.67184000 & -3.68043700 & 0.54519500 \\ \mathrm{C} & -0.49478200 & 1.68911100 & -0.20809200 \\ \mathrm{C} & -1.59377100 & 2.15944200 & -0.94422700 \\ \mathrm{C} & 0.20001700 & 2.58233300 & 0.62252400 \\ \mathrm{C} & -1.97832700 & 3.48811700 & -0.85364200 \\ \mathrm{H} & -2.12790100 & 1.47689600 & -1.60055900 \\ \mathrm{C} & -0.19946500 & 3.90631900 & 0.71381400 \\ \mathrm{H} & 1.04632200 & 2.23117700 & 1.20899800 \\ \mathrm{C} & -1.28664800 & 4.36647400 & -0.02449300 \\ \mathrm{H} & -2.82478900 & 3.84090400 & -1.43735700 \\ \mathrm{H} & 0.34410700 & 4.58477500 & 1.36625000 \\ \mathrm{H} & -1.59220900 & 5.40723100 & 0.04482500 \\ \mathrm{C} & -0.08126700 & 0.30490000 & -0.33728500 \\ \mathrm{H} & 0.68685800 & -0.11697200 & 0.99193300\end{array}$

(td)-E-(f) (-1015.134468 a.u.)

$\begin{array}{lrrr}\text { C } & 0.66186800 & -1.39709400 & -0.77068000 \\ \mathrm{C} & 1.88496400 & -0.98187600 & -1.48754100 \\ \mathrm{O} & 1.60021100 & -0.62962500 & -2.75582500 \\ \mathrm{C} & 2.70976100 & -0.12564900 & -3.49175700 \\ \mathrm{H} & 3.50879300 & -0.87044800 & -3.55413000 \\ \mathrm{H} & 3.11190300 & 0.77248800 & -3.01028900 \\ \mathrm{H} & 2.33169100 & 0.11236900 & -4.48652300 \\ \mathrm{O} & 2.99540600 & -0.92544200 & -1.01160400 \\ \mathrm{O} & -1.43847500 & -2.11735500 & 0.72730500 \\ \mathrm{~N} & -1.13483700 & -0.72265200 & 0.87956600 \\ \mathrm{C} & -2.06934400 & 0.03284800 & 0.10928000 \\ \mathrm{C} & -3.83469300 & 1.63411500 & -1.33822300 \\ & & & \end{array}$




$\begin{array}{lrrc}\mathrm{C} & -2.86699000 & -0.53320300 & -0.88448400 \\ \mathrm{C} & -2.17484800 & 1.39624500 & 0.39606400 \\ \mathrm{C} & -3.74956800 & 0.27154200 & -1.59762800 \\ \mathrm{C} & -3.04247200 & 2.19202200 & -0.33839400 \\ \mathrm{H} & -2.80480500 & -1.59897400 & -1.07769300 \\ \mathrm{H} & -1.57553700 & 1.81706700 & 1.20026800 \\ \mathrm{H} & -4.37442600 & -0.17613400 & -2.36729000 \\ \mathrm{H} & -3.11338200 & 3.25355800 & -0.11311800 \\ \mathrm{C} & -0.26592200 & -2.81424700 & 1.14344100 \\ \mathrm{H} & -0.32909200 & -3.78975300 & 0.64121900 \\ \mathrm{H} & -4.52482200 & 2.25730500 & -1.90163000 \\ \mathrm{C} & 0.85432600 & -1.95263200 & 0.61619000 \\ \mathrm{H} & -0.16173100 & -1.76264100 & -1.38142100 \\ \mathrm{C} & -0.20924700 & -2.97702400 & 2.64803500 \\ \mathrm{H} & 0.70826200 & -3.49408500 & 2.95074500 \\ \mathrm{H} & -0.23425600 & -1.99511800 & 3.13377300 \\ \mathrm{H} & -1.06853100 & -3.55408300 & 3.00202400 \\ \mathrm{C} & 0.91847800 & 0.68241000 & 0.84925400 \\ \mathrm{C} & 1.12111500 & 1.68638600 & -0.09639300 \\ \mathrm{C} & 1.24523600 & 0.91968900 & 2.18364700 \\ \mathrm{C} & 1.65556900 & 2.91091600 & 0.28613200 \\ \mathrm{H} & 0.83802700 & 1.50523900 & -1.13317900 \\ \mathrm{C} & 1.77387400 & 2.14494800 & 2.56838200 \\ \mathrm{H} & 1.07554700 & 0.13275200 & 2.91738900 \\ \mathrm{C} & 1.98032900 & 3.14199000 & 1.61866600 \\ \mathrm{H} & 1.81162900 & 3.68925500 & -0.45683900 \\ \mathrm{H} & 2.02818200 & 2.32327000 & 3.61043000 \\ \mathrm{H} & 2.39578700 & 4.10098100 & 1.91910900 \\ \mathrm{C} & 0.27190700 & -0.59945000 & 0.47028800 \\ \mathrm{H} & 1.87742600 & -2.07672100 & 0.96552700\end{array}$

TS(td)-VIII-(f) (-1015.053048 a.u.)

$\begin{array}{lccc}\mathrm{C} & 2.52744400 & 0.33172800 & -0.09523100 \\ \mathrm{C} & 3.68990100 & -0.47629900 & 0.09474800 \\ \mathrm{O} & 4.80987100 & 0.29553600 & 0.14286100 \\ \mathrm{C} & 6.01772100 & -0.42953800 & 0.29312500 \\ \mathrm{H} & 6.16290700 & -1.13470000 & -0.53233800 \\ \mathrm{H} & 6.02524600 & -0.99846500 & 1.22925200 \\ \mathrm{H} & 6.81903500 & 0.31158200 & 0.29801900 \\ \mathrm{O} & 3.73401800 & -1.69670800 & 0.20534200 \\ \mathrm{O} & -0.22447000 & -1.71685300 & -1.33846400 \\ \mathrm{~N} & -0.96984800 & -0.62279600 & -0.85553800 \\ \mathrm{C} & -2.17852400 & -1.00541800 & -0.21897100 \\ \mathrm{C} & -4.59728400 & -1.77797000 & 0.93111000 \\ & & & \end{array}$




$\begin{array}{lccc}\mathrm{C} & -2.91927700 & -2.04633700 & -0.78406900 \\ \mathrm{C} & -2.64350100 & -0.35616400 & 0.92638100 \\ \mathrm{C} & -4.11768900 & -2.43036000 & -0.20193300 \\ \mathrm{C} & -3.85532400 & -0.74257200 & 1.48736100 \\ \mathrm{H} & -2.53526900 & -2.54890800 & -1.66628100 \\ \mathrm{H} & -2.05857300 & 0.43923500 & 1.38054300 \\ \mathrm{H} & -4.68536800 & -3.24679600 & -0.64188500 \\ \mathrm{H} & -4.21106700 & -0.23424100 & 2.38043900 \\ \mathrm{C} & 0.96459300 & -1.76067300 & -0.52533600 \\ \mathrm{H} & 1.75870000 & -2.14226300 & -1.17166600 \\ \mathrm{H} & -5.54033400 & -2.07887600 & 1.37977300 \\ \mathrm{C} & 1.18111200 & -0.28303600 & -0.18733300 \\ \mathrm{H} & 2.60771400 & 1.41213400 & -0.04944200 \\ \mathrm{C} & 0.76867600 & -2.63244000 & 0.69457500 \\ \mathrm{H} & 1.70671100 & -2.67879800 & 1.25740000 \\ \mathrm{H} & -0.02393100 & -2.22515800 & 1.33585300 \\ \mathrm{H} & 0.48354400 & -3.64652100 & 0.39899500 \\ \mathrm{C} & -0.47030100 & 1.71291400 & -0.24526500 \\ \mathrm{C} & -1.58366300 & 2.20267800 & -0.95179700 \\ \mathrm{C} & 0.23266300 & 2.59760900 & 0.59019000 \\ \mathrm{C} & -1.96657400 & 3.52821100 & -0.83259200 \\ \mathrm{H} & -2.12907700 & 1.52927100 & -1.60823700 \\ \mathrm{C} & -0.16165000 & 3.92215500 & 0.70838700 \\ \mathrm{H} & 1.08032700 & 2.24349900 & 1.17259400 \\ \mathrm{C} & -1.25913100 & 4.39591700 & -0.00355600 \\ \mathrm{H} & -2.82356300 & 3.89014700 & -1.39537500 \\ \mathrm{H} & 0.39287800 & 4.58872000 & 1.36426500 \\ \mathrm{H} & -1.56178200 & 5.43602000 & 0.08606000 \\ \mathrm{C} & -0.07565500 & 0.32873600 & -0.38715200 \\ \mathrm{H} & 1.44427500 & -0.08695400 & 0.93702600\end{array}$

TS(td)-VI-(c) (-1992.71062 a.u.)

$\begin{array}{lrrr}\text { C } & 1.33056900 & 1.94371900 & 0.07854900 \\ \mathrm{C} & 1.16258400 & 3.38921400 & 0.34252600 \\ \mathrm{O} & 0.33441800 & 3.96596800 & -0.53803700 \\ \mathrm{C} & 0.08422400 & 5.34911000 & -0.31504200 \\ \mathrm{H} & 1.02001700 & 5.91655200 & -0.31177700 \\ \mathrm{H} & -0.41791700 & 5.49692800 & 0.64623000 \\ \mathrm{H} & -0.55896900 & 5.67581900 & -1.13247200 \\ \mathrm{O} & 1.69787200 & 3.96952500 & 1.26266200 \\ \mathrm{O} & 1.91501600 & -0.58876000 & 2.37172700 \\ \mathrm{~N} & 2.64909000 & -1.03382900 & 1.23017100 \\ \mathrm{C} & 2.17467300 & -2.29611400 & 0.80573600 \\ \mathrm{C} & 1.25784600 & -4.79353400 & -0.06694300 \\ & & & \end{array}$




\begin{tabular}{|c|c|c|c|}
\hline C & 1.29338100 & -3.03547100 & 1.59721300 \\
\hline C & 2.62090500 & -2.82348100 & -0.41191000 \\
\hline C & 0.83439300 & -4.27056700 & 1.15033000 \\
\hline C & 2.16077400 & -4.06243800 & -0.83724100 \\
\hline $\mathrm{H}$ & 0.96583900 & -2.63085600 & 2.54791200 \\
\hline $\mathrm{H}$ & 3.32207000 & -2.26178700 & -1.02355100 \\
\hline $\mathrm{H}$ & 0.13721500 & -4.83067100 & 1.77035500 \\
\hline $\mathrm{H}$ & 2.51710600 & -4.46029200 & -1.78530100 \\
\hline C & 2.05900600 & 0.84196600 & 2.44904000 \\
\hline $\mathrm{H}$ & 3.05389600 & 1.07818200 & 2.87542100 \\
\hline $\mathrm{H}$ & 0.89839500 & -5.76111400 & -0.40748100 \\
\hline $\mathrm{Rh}$ & -3.18903800 & -0.79137400 & -0.48328100 \\
\hline $\mathrm{O}$ & -1.98869600 & -2.22003900 & -1.33638500 \\
\hline C & -0.75073900 & -1.99059000 & -1.39665500 \\
\hline $\mathrm{O}$ & -0.13918000 & -0.95031200 & -1.01846700 \\
\hline $\mathrm{Rh}$ & -1.21385200 & 0.50413600 & -0.06344400 \\
\hline $\mathrm{O}$ & -1.04487000 & -0.52992900 & 1.70373600 \\
\hline C & -1.91593800 & -1.39536400 & 1.98678000 \\
\hline $\mathrm{O}$ & -2.93171700 & -1.72420200 & 1.31621500 \\
\hline $\mathrm{H}$ & -1.77290200 & -1.92763800 & 2.94072700 \\
\hline $\mathrm{O}$ & -1.52067100 & 1.44173700 & -1.86718800 \\
\hline C & -2.50619000 & 1.06603000 & -2.56025500 \\
\hline $\mathrm{O}$ & -3.36045600 & 0.18359600 & -2.28093500 \\
\hline $\mathrm{H}$ & -2.63297100 & 1.57552000 & -3.52845100 \\
\hline $\mathrm{O}$ & -2.38329400 & 1.90621000 & 0.87561900 \\
\hline C & -3.62801000 & 1.69449600 & 0.89686800 \\
\hline $\mathrm{O}$ & -4.25967100 & 0.71035100 & 0.42653900 \\
\hline $\mathrm{H}$ & -4.23775100 & 2.46860900 & 1.38901700 \\
\hline $\mathrm{H}$ & -0.11881200 & -2.78669300 & -1.82240300 \\
\hline C & 2.05230100 & 1.13899700 & 0.98806500 \\
\hline $\mathrm{H}$ & 0.52057200 & 1.53412200 & -0.55382400 \\
\hline C & 0.96359000 & 1.41959600 & 3.30240100 \\
\hline $\mathrm{H}$ & 1.13217300 & 2.49553300 & 3.40862200 \\
\hline $\mathrm{H}$ & -0.01590100 & 1.25223700 & 2.84399600 \\
\hline $\mathrm{H}$ & 0.97056900 & 0.95952700 & 4.29539700 \\
\hline C & 3.90794800 & 0.19164500 & -0.52777400 \\
\hline C & 3.88099700 & 0.30056000 & -1.91765700 \\
\hline C & 5.14257000 & 0.17192600 & 0.13190700 \\
\hline C & 5.06443400 & 0.43722700 & -2.63797600 \\
\hline $\mathrm{H}$ & 2.92345200 & 0.25775800 & -2.43655400 \\
\hline C & 6.32169200 & 0.30051900 & -0.58846800 \\
\hline $\mathrm{H}$ & 5.16264600 & 0.05195500 & 1.21332600 \\
\hline C & 6.28516200 & 0.44024000 & -1.97465300 \\
\hline $\mathrm{H}$ & 5.02891100 & 0.52700100 & -3.72098200 \\
\hline
\end{tabular}




$\begin{array}{rrrr}H & 7.27522700 & 0.29621200 & -0.06547800 \\ H & 7.21109500 & 0.54034800 & -2.53612400 \\ \mathrm{C} & 2.66284700 & 0.07088100 & 0.30547800 \\ \mathrm{H} & 1.89832300 & 0.85067600 & -0.74096600\end{array}$

TS(td)-VII-(c) (-1992.798897 a.u.)

\begin{tabular}{lrrr} 
C & -0.00016300 & 1.94841400 & 0.46227500 \\
$\mathrm{C}$ & 0.81195000 & 3.12757800 & 0.14692600 \\
$\mathrm{O}$ & 1.55808000 & 3.47545300 & 1.21339400 \\
$\mathrm{C}$ & 2.56735800 & 4.43773300 & 0.94908300 \\
$\mathrm{H}$ & 2.13910700 & 5.36319300 & 0.55149800 \\
$\mathrm{H}$ & 3.28686500 & 4.04069300 & 0.22438900 \\
$\mathrm{H}$ & 3.06274000 & 4.62655900 & 1.90267700 \\
$\mathrm{O}$ & 0.84892400 & 3.73496500 & -0.90793600 \\
$\mathrm{O}$ & -3.02162300 & 1.94812400 & -1.75888000 \\
$\mathrm{~N}$ & -3.49191000 & 1.32397600 & -0.57388000 \\
$\mathrm{C}$ & -4.28769100 & 0.17193100 & -0.90717500 \\
$\mathrm{C}$ & -5.72890900 & -2.14077400 & -1.37969900 \\
$\mathrm{C}$ & -3.90544900 & -0.66758800 & -1.95165300 \\
$\mathrm{C}$ & -5.39127400 & -0.11502500 & -0.11576000 \\
$\mathrm{C}$ & -4.63494000 & -1.82634800 & -2.18442100 \\
$\mathrm{C}$ & -6.10669700 & -1.28559200 & -0.34971200 \\
$\mathrm{H}$ & -3.05214600 & -0.39981800 & -2.57069300 \\
$\mathrm{H}$ & -5.66226700 & 0.56982500 & 0.68422000 \\
$\mathrm{H}$ & -4.34813300 & -2.48885200 & -2.99769700 \\
$\mathrm{H}$ & -6.96579200 & -1.52459000 & 0.27182500 \\
$\mathrm{C}$ & -1.67657200 & 2.44531200 & -1.54987400 \\
$\mathrm{H}$ & -1.71015800 & 3.53388800 & -1.38438200 \\
$\mathrm{H}$ & -6.29340300 & -3.05146900 & -1.56380100 \\
$\mathrm{Rh}$ & 2.60045500 & -1.89387600 & -0.31530800 \\
$\mathrm{O}$ & 1.98380600 & -2.45531900 & 1.57845500 \\
$\mathrm{C}$ & 1.24437700 & -1.67194300 & 2.22435800 \\
$\mathrm{O}$ & 0.78927100 & -0.54435000 & 1.87767200 \\
$\mathrm{Rh}$ & 1.28793000 & 0.16459300 & 0.00080500 \\
$\mathrm{O}$ & -0.31639900 & -0.89268000 & -0.81961900 \\
$\mathrm{C}$ & -0.14843000 & -2.10341400 & -1.17508600 \\
$\mathrm{O}$ & 0.89585900 & -2.78097500 & -1.11307600 \\
$\mathrm{H}$ & -1.04696400 & -2.59782600 & -1.58607700 \\
$\mathrm{O}$ & 2.98362600 & 1.03489800 & 0.75999500 \\
$\mathrm{C}$ & 4.02563300 & 0.32337200 & 0.83484700 \\
$\mathrm{O}$ & 4.18867500 & -0.87686100 & 0.50075200 \\
$\mathrm{H}$ & 0.83380200 & 1.25290400 \\
$\mathrm{O}$ & 0.73259100 & -1.88883100 \\
& & -0.04536000 & -2.52703600 \\
\hline
\end{tabular}




$\begin{array}{cccc}\mathrm{O} & 3.10463600 & -1.16272400 & -2.17883000 \\ \mathrm{H} & 2.94732200 & 0.31121000 & -3.52694000 \\ \mathrm{H} & 0.94468400 & -2.00685100 & 3.23275800 \\ \mathrm{C} & -1.25351800 & 1.78717500 & -0.25049800 \\ \mathrm{H} & -0.07327700 & 1.75677300 & 1.53409300 \\ \mathrm{C} & -0.87932600 & 2.10604800 & -2.78557600 \\ \mathrm{H} & 0.13180000 & 2.50732100 & -2.70089600 \\ \mathrm{H} & -0.82073300 & 1.01803500 & -2.90767500 \\ \mathrm{H} & -1.37583500 & 2.53368300 & -3.66223300 \\ \mathrm{C} & -2.49750100 & 0.41771200 & 1.53794200 \\ \mathrm{C} & -2.64813500 & -0.96962900 & 1.59299700 \\ \mathrm{C} & -2.38124000 & 1.15940000 & 2.71473500 \\ \mathrm{C} & -2.67056800 & -1.60952600 & 2.82507900 \\ \mathrm{H} & -2.73288300 & -1.54351800 & 0.67178400 \\ \mathrm{C} & -2.41619800 & 0.51453200 & 3.94424000 \\ \mathrm{H} & -2.25668700 & 2.23938300 & 2.65992000 \\ \mathrm{C} & -2.55337000 & -0.86929300 & 3.99832900 \\ \mathrm{H} & -2.77751300 & -2.69036100 & 2.86854100 \\ \mathrm{H} & -2.32535400 & 1.09238700 & 4.86015300 \\ \mathrm{H} & -2.56909000 & -1.37461000 & 4.96109700 \\ \mathrm{C} & -2.39907900 & 1.12861500 & 0.24742700 \\ \mathrm{H} & -1.41764500 & 0.51605400 & -0.52096000\end{array}$

(td)-E-(c) (-1992.833833 a.u.)

$\begin{array}{lccc}\text { C } & 0.36133900 & -0.71618600 & 0.94350600 \\ \text { C } & 0.03546200 & -1.88228500 & 1.76458300 \\ \text { O } & -0.32841600 & -1.50452700 & 3.01070100 \\ \text { C } & -0.91203300 & -2.52834800 & 3.79944400 \\ \text { H } & -0.22462400 & -3.37137800 & 3.92655500 \\ \text { H } & -1.83270200 & -2.89010500 & 3.32963500 \\ \text { H } & -1.13689000 & -2.07487600 & 4.76651700 \\ \text { O } & 0.03580000 & -3.05279300 & 1.41584200 \\ \text { O } & 3.30463500 & -1.99358800 & -0.99455500 \\ \text { N } & 3.65519900 & -0.76776100 & -0.48477100 \\ \text { C } & 5.01385300 & -0.41578400 & -0.74628500 \\ \text { C } & 7.64325600 & 0.21986000 & -1.26475400 \\ \text { C } & 6.00718900 & -1.32712800 & -0.40441800 \\ \text { C } & 5.30419700 & 0.79617700 & -1.36441000 \\ \text { C } & 7.33090900 & -0.99739000 & -0.66564700 \\ \text { C } & 6.63269900 & 1.11171200 & -1.61558200 \\ H & 5.73419500 & -2.27448700 & 0.05150400 \\ \text { H } & 4.50106800 & 1.47504900 & -1.63947300 \\ \text { H } & 8.12044900 & -1.69571300 & -0.40205100 \\ \text { H } & 6.87750900 & 2.05411700 & -2.09766100 \\ & & & \\ & & 568 & \end{array}$




\begin{tabular}{|c|c|c|c|}
\hline C & 1.97769300 & -2.29253100 & -0.41941100 \\
\hline $\mathrm{H}$ & 2.15962200 & -2.81932400 & 0.52650100 \\
\hline $\mathrm{H}$ & 8.68078100 & 0.47233800 & -1.46764100 \\
\hline $\mathrm{Rh}$ & -1.53914100 & -0.10607000 & 0.08728600 \\
\hline $\mathrm{O}$ & -1.58908000 & -1.67717300 & -1.24845500 \\
\hline C & -2.57944800 & -1.74956000 & -2.03464000 \\
\hline $\mathrm{O}$ & -3.56184600 & -0.97812400 & -2.14501100 \\
\hline $\mathrm{Rh}$ & -3.67934800 & 0.67616000 & -0.89764100 \\
\hline $\mathrm{O}$ & -2.54003500 & 1.76953400 & -2.24956700 \\
\hline C & -1.29547100 & 1.70817500 & -2.17888700 \\
\hline $\mathrm{O}$ & -0.57415200 & 1.05445700 & -1.36077800 \\
\hline $\mathrm{H}$ & -0.73251100 & 2.29932300 & -2.92379000 \\
\hline $\mathrm{O}$ & -4.63902000 & -0.48031300 & 0.51557400 \\
\hline C & -3.93510700 & -1.13991800 & 1.31772800 \\
\hline $\mathrm{O}$ & -2.67579800 & -1.20091300 & 1.41110500 \\
\hline $\mathrm{H}$ & -4.48354900 & -1.75688100 & 2.05151700 \\
\hline $\mathrm{O}$ & -3.57856900 & 2.24432500 & 0.46262200 \\
\hline C & -2.60120600 & 2.30681000 & 1.24588500 \\
\hline $\mathrm{O}$ & -1.60290200 & 1.53416700 & 1.34426200 \\
\hline $\mathrm{H}$ & -2.59987000 & 3.15697300 & 1.95196800 \\
\hline $\mathrm{H}$ & -2.56509000 & -2.61712800 & -2.71822400 \\
\hline C & 1.39617900 & -0.89633500 & -0.16335700 \\
\hline $\mathrm{H}$ & 0.99119900 & -0.51362700 & -1.12527900 \\
\hline C & 1.25640800 & -3.16179000 & -1.40536100 \\
\hline $\mathrm{H}$ & 0.28622600 & -3.42363600 & -0.97763900 \\
\hline $\mathrm{H}$ & 1.09889600 & -2.62502500 & -2.34713100 \\
\hline $\mathrm{H}$ & 1.82745500 & -4.07481400 & -1.60028400 \\
\hline C & 2.79857900 & 1.18473600 & 0.69319700 \\
\hline C & 3.90421600 & 1.47234600 & 1.50858600 \\
\hline C & 1.76863700 & 2.12530700 & 0.54866000 \\
\hline C & 3.97879700 & 2.69181200 & 2.16122500 \\
\hline $\mathrm{H}$ & 4.68585300 & 0.72928400 & 1.65181700 \\
\hline C & 1.86600800 & 3.35112600 & 1.19278000 \\
\hline $\mathrm{H}$ & 0.90318600 & 1.89660900 & -0.07185100 \\
\hline C & 2.96370600 & 3.63421300 & 1.99845000 \\
\hline $\mathrm{H}$ & 4.82571000 & 2.90641000 & 2.80747800 \\
\hline $\mathrm{H}$ & 1.06489100 & 4.07641500 & 1.07820000 \\
\hline $\mathrm{H}$ & 3.02603700 & 4.58899000 & 2.51501000 \\
\hline C & 2.66016700 & -0.10564800 & 0.03858200 \\
\hline $\mathrm{H}$ & 0.57665000 & 0.14512200 & 1.58054200 \\
\hline
\end{tabular}

TS(td)-VIII-(c) (-1992.78241 a.u.)

$\begin{array}{lrrr}\text { C } & -0.20800000 & 1.56155300 & 0.93802100 \\ \text { C } & 0.48074000 & 2.86050500 & 0.82771200 \\ & & & \\ & & & \end{array}$




\begin{tabular}{|c|c|c|c|}
\hline $\mathrm{O}$ & 1.14083600 & 3.12977300 & 1.96355700 \\
\hline C & 1.98232700 & 4.27620800 & 1.90737700 \\
\hline $\mathrm{H}$ & 1.39961600 & 5.17790100 & 1.69528200 \\
\hline $\mathrm{H}$ & 2.73893000 & 4.14936400 & 1.12673900 \\
\hline $\mathrm{H}$ & 2.45473800 & 4.35033600 & 2.88755200 \\
\hline $\mathrm{O}$ & 0.47057700 & 3.59357800 & -0.14002800 \\
\hline $\mathrm{O}$ & -3.47443800 & 2.24770200 & -0.78049000 \\
\hline$N$ & -3.72816500 & 1.02872200 & -0.12991500 \\
\hline C & -4.60226300 & 0.16171100 & -0.83610300 \\
\hline C & -6.36875200 & -1.48409700 & -2.20396600 \\
\hline C & -5.81254400 & 0.68016200 & -1.29511500 \\
\hline C & -4.26101700 & -1.17013100 & -1.07022000 \\
\hline C & -6.68741400 & -0.14556300 & -1.98685800 \\
\hline C & -5.15749500 & -1.99112500 & -1.74392100 \\
\hline $\mathrm{H}$ & -6.04346300 & 1.72617800 & -1.11283300 \\
\hline $\mathrm{H}$ & -3.29882500 & -1.55299000 & -0.73464900 \\
\hline $\mathrm{H}$ & -7.62890100 & 0.25718200 & -2.35209200 \\
\hline $\mathrm{H}$ & -4.89609100 & -3.03069600 & -1.92597600 \\
\hline C & -2.03449100 & 2.35616500 & -0.89903100 \\
\hline $\mathrm{H}$ & -1.80080700 & 3.40829400 & -0.71593600 \\
\hline $\mathrm{H}$ & -7.06134600 & -2.12944700 & -2.73827800 \\
\hline $\mathrm{Rh}$ & 1.42968900 & 0.15382400 & 0.03150600 \\
\hline $\mathrm{O}$ & 1.50810200 & 0.99312900 & -1.84900200 \\
\hline C & 2.35075500 & 0.54041900 & -2.67701200 \\
\hline $\mathrm{O}$ & 3.16831300 & -0.40087000 & -2.52947300 \\
\hline $\mathrm{Rh}$ & 3.19578800 & -1.38509400 & -0.72026000 \\
\hline $\mathrm{O}$ & 1.69003700 & -2.64154200 & -1.38580400 \\
\hline C & 0.49926100 & -2.29589000 & -1.22401300 \\
\hline $\mathrm{O}$ & 0.04909900 & -1.24391900 & -0.67317500 \\
\hline $\mathrm{H}$ & -0.27153400 & -2.98870800 & -1.60210900 \\
\hline $\mathrm{O}$ & 4.56999700 & -0.02454300 & -0.01981100 \\
\hline C & 4.13706600 & 1.04758200 & 0.46951500 \\
\hline $\mathrm{O}$ & 2.93920900 & 1.41928500 & 0.62880100 \\
\hline $\mathrm{H}$ & 4.89858400 & 1.76888800 & 0.81141100 \\
\hline $\mathrm{O}$ & 3.10510800 & -2.24749400 & 1.15649400 \\
\hline C & 2.31492900 & -1.76403600 & 2.00114400 \\
\hline $\mathrm{O}$ & 1.50351600 & -0.80051000 & 1.86566500 \\
\hline $\mathrm{H}$ & 2.31653700 & -2.23643800 & 2.99853300 \\
\hline $\mathrm{H}$ & 2.36042000 & 1.04521600 & -3.65724600 \\
\hline C & -1.54934300 & 1.45593200 & 0.23481800 \\
\hline $\mathrm{H}$ & -0.61412700 & 0.72225200 & -0.12644700 \\
\hline C & -1.57408200 & 1.91180300 & -2.26714400 \\
\hline $\mathrm{H}$ & -0.48718300 & 2.01836800 & -2.34750500 \\
\hline $\mathrm{H}$ & -1.83442100 & 0.85559700 & -2.42503100 \\
\hline
\end{tabular}




$\begin{array}{lrrr}\mathrm{H} & -2.05460500 & 2.51291800 & -3.04510000 \\ \mathrm{C} & -2.77803700 & -0.33626000 & 1.65691900 \\ \mathrm{C} & -4.01301500 & -0.46486700 & 2.31343800 \\ \mathrm{C} & -1.70466100 & -1.14089100 & 2.05803200 \\ \mathrm{C} & -4.16255800 & -1.37390500 & 3.34856100 \\ \mathrm{H} & -4.84441400 & 0.16962800 & 2.01558300 \\ \mathrm{C} & -1.86376800 & -2.04904600 & 3.09651500 \\ \mathrm{H} & -0.75197500 & -1.08766400 & 1.54357400 \\ \mathrm{C} & -3.08841100 & -2.16888900 & 3.74437200 \\ \mathrm{H} & -5.12060900 & -1.45862400 & 3.85530800 \\ \mathrm{H} & -1.01890100 & -2.66543100 & 3.39563500 \\ \mathrm{H} & -3.20835000 & -2.87944100 & 4.55870800 \\ \mathrm{C} & -2.63819200 & 0.66325900 & 0.60378100 \\ \mathrm{H} & -0.21166800 & 1.09310300 & 1.92340800\end{array}$

TS(td)-VI-(o) (-1992.72297 a.u.)

$\begin{array}{lccc}\text { C } & -1.90720700 & 0.58302800 & 1.95423000 \\ \text { C } & -0.49586700 & 0.96385200 & 2.01883500 \\ \text { O } & -0.08817900 & 1.32936000 & 3.22400100 \\ \text { C } & 1.27445800 & 1.74541800 & 3.35426600 \\ \text { H } & 1.52922900 & 2.48595300 & 2.59123300 \\ \text { H } & 1.94106200 & 0.88501800 & 3.24670300 \\ \text { H } & 1.35967100 & 2.17298300 & 4.35359100 \\ \text { O } & 0.20933400 & 0.95119800 & 1.00896200 \\ \text { O } & -3.35678500 & -1.99341900 & 0.12660600 \\ \text { N } & -4.20748700 & -0.89121200 & -0.18379900 \\ \text { C } & -5.56349400 & -1.24444000 & -0.13252800 \\ \text { C } & -8.26840200 & -1.94040200 & -0.15128800 \\ \text { C } & -5.94949800 & -2.54335800 & -0.47540800 \\ \text { C } & -6.53284100 & -0.29653400 & 0.20944100 \\ \text { C } & -7.29568200 & -2.88240600 & -0.47327900 \\ \text { C } & -7.87617800 & -0.64875900 & 0.18493000 \\ \text { H } & -5.18810200 & -3.27205900 & -0.73391000 \\ \text { H } & -6.23730500 & 0.71149000 & 0.48827200 \\ \text { H } & -7.58607800 & -3.89783200 & -0.73398100 \\ \text { H } & -8.62260200 & 0.09741800 & 0.44827800 \\ \text { C } & -2.01533200 & -1.46367100 & 0.17476500 \\ \text { H } & -1.62224300 & -1.32884700 & -0.85009100 \\ \text { H } & -9.32107300 & -2.21128100 & -0.15829600 \\ \text { Rh } & 4.14829100 & -0.68316900 & -0.82595300 \\ \text { O } & 4.26953000 & -1.94282300 & 0.80241900 \\ \mathrm{C} & 3.37635700 & -1.87078000 & 1.68363700 \\ \text { O } & 2.37905000 & -1.09297800 & 1.72489200 \\ \text { Rh } & 2.16824500 & 0.25538400 & 0.17768300\end{array}$




\begin{tabular}{lccc} 
O & 1.07147200 & -1.12543100 & -0.88434100 \\
$\mathrm{C}$ & 1.69896800 & -1.95323600 & -1.60241600 \\
$\mathrm{O}$ & 2.94092900 & -2.04511100 & -1.77957700 \\
$\mathrm{H}$ & 1.07517300 & -2.68806300 & -2.13869400 \\
$\mathrm{O}$ & 3.40132600 & 1.59279600 & 1.15421600 \\
$\mathrm{C}$ & 4.64101400 & 1.53358400 & 0.91880700 \\
$\mathrm{O}$ & 5.25405000 & 0.72913000 & 0.17109600 \\
$\mathrm{H}$ & 5.26222200 & 2.28144500 & 1.43810600 \\
$\mathrm{O}$ & 2.05594800 & 1.54243800 & -1.41422200 \\
$\mathrm{C}$ & 2.92741900 & 1.43425600 & -2.32011100 \\
$\mathrm{O}$ & 3.89645800 & 0.63050000 & -2.38079300 \\
$\mathrm{H}$ & 2.82604100 & 2.13352000 & -3.16563900 \\
$\mathrm{H}$ & 3.46940100 & -2.57158100 & 2.52924200 \\
$\mathrm{C}$ & -2.33085000 & -0.14726600 & 0.80905100 \\
$\mathrm{H}$ & -2.36628300 & 0.37967100 & 2.92329600 \\
$\mathrm{C}$ & -1.16095900 & -2.40653400 & 0.97812700 \\
$\mathrm{H}$ & -0.16514200 & -1.96975400 & 1.10869800 \\
$\mathrm{H}$ & -1.61521600 & -2.57634100 & 1.96025100 \\
$\mathrm{H}$ & -1.05194600 & -3.36891600 & 0.46801600 \\
$\mathrm{C}$ & -3.86403500 & 1.55380000 & -0.31427600 \\
$\mathrm{C}$ & -4.33750400 & 2.70555600 & 0.31076700 \\
$\mathrm{C}$ & -3.59995100 & 1.58081600 & -1.68769300 \\
$\mathrm{C}$ & -4.51929200 & 3.87841800 & -0.41585100 \\
$\mathrm{H}$ & -4.57294500 & 2.68057900 & 1.37423100 \\
$\mathrm{C}$ & -3.78744900 & 2.74971300 & -2.41238100 \\
$\mathrm{H}$ & -3.24822200 & 0.67473800 & -2.17683100 \\
$\mathrm{C}$ & -4.24422500 & 3.90250700 & -1.77767700 \\
$\mathrm{H}$ & -4.88691600 & 4.77078300 & 0.08493000 \\
$\mathrm{H}$ & -3.56976700 & 2.76273500 & -3.47771400 \\
$\mathrm{H}$ & -4.39097000 & 4.81676200 & -2.34785300 \\
$\mathrm{C}$ & -3.62149300 & 0.25733600 & 0.41825300 \\
$\mathrm{H}$ & -3.36253600 & 0.96969700 & 1.68757800 \\
& & & \\
& & & \\
\hline & & & \\
& & &
\end{tabular}

TS(td)-VII-(o) (-1992.784682 a.u.)

$\begin{array}{lrrr}\text { C } & -0.98352300 & 2.38832900 & -1.25700900 \\ \mathrm{C} & 0.31506900 & 2.31279700 & -1.76373900 \\ \mathrm{O} & 0.96487000 & 3.48741600 & -1.81905300 \\ \mathrm{C} & 2.32862800 & 3.43966200 & -2.21938200 \\ \mathrm{H} & 2.43933800 & 2.94906400 & -3.19131200 \\ \mathrm{H} & 2.93271000 & 2.89685900 & -1.48514400 \\ \mathrm{H} & 2.65504800 & 4.47936900 & -2.28145800 \\ \mathrm{O} & 0.88121900 & 1.24851500 & -2.15266400 \\ \mathrm{O} & -3.50785000 & 0.17107400 & -2.48480100 \\ \mathrm{~N} & -3.85562100 & 0.19159100 & -1.11522300 \\ & & & \end{array}$




\begin{tabular}{|c|c|c|c|}
\hline C & -4.34660500 & -1.05189700 & -0.62760200 \\
\hline C & -5.36331800 & -3.48441200 & 0.26489400 \\
\hline C & -5.18126000 & -1.80077500 & -1.45736700 \\
\hline C & -4.01269800 & -1.51855800 & 0.64504700 \\
\hline C & -5.67911200 & -3.01547000 & -1.00788500 \\
\hline C & -4.53231400 & -2.73075800 & 1.08590000 \\
\hline $\mathrm{H}$ & -5.41331900 & -1.42874100 & -2.45046600 \\
\hline $\mathrm{H}$ & -3.35813200 & -0.93823300 & 1.29143400 \\
\hline $\mathrm{H}$ & -6.32290000 & -3.60063400 & -1.66000700 \\
\hline $\mathrm{H}$ & -4.26983700 & -3.09063700 & 2.07787300 \\
\hline C & -2.06861300 & 0.32331400 & -2.50746100 \\
\hline $\mathrm{H}$ & -1.84334500 & 0.87291600 & -3.42681000 \\
\hline $\mathrm{H}$ & -5.75889600 & -4.43520700 & 0.61229300 \\
\hline $\mathrm{Rh}$ & 1.79990700 & 0.08780900 & -0.50273800 \\
\hline $\mathrm{O}$ & 1.90237800 & 1.72500300 & 0.75013200 \\
\hline C & 2.42216700 & 1.56052100 & 1.89026400 \\
\hline $\mathrm{O}$ & 2.89993300 & 0.50921000 & 2.38783100 \\
\hline $\mathrm{Rh}$ & 2.88475900 & -1.20612900 & 1.24276800 \\
\hline $\mathrm{O}$ & 4.67772200 & -0.67035900 & 0.40543500 \\
\hline C & 4.66027500 & 0.04912300 & -0.62681900 \\
\hline $\mathrm{O}$ & 3.66080100 & 0.52033700 & -1.23880800 \\
\hline $\mathrm{H}$ & 5.64254200 & 0.30589100 & -1.05572300 \\
\hline $\mathrm{O}$ & 1.01603900 & -1.68395100 & 1.98556200 \\
\hline C & 0.01619600 & -1.20155500 & 1.40798300 \\
\hline $\mathrm{O}$ & -0.00958300 & -0.43954300 & 0.39558900 \\
\hline $\mathrm{H}$ & -0.97115000 & -1.46799700 & 1.82707200 \\
\hline $\mathrm{O}$ & 2.79439300 & -2.83077600 & -0.01409300 \\
\hline C & 2.26786000 & -2.67372500 & -1.14669900 \\
\hline $\mathrm{O}$ & 1.76687900 & -1.62554400 & -1.64105100 \\
\hline $\mathrm{H}$ & 2.23722000 & -3.56568500 & -1.79376100 \\
\hline $\mathrm{H}$ & 2.45626300 & 2.45906500 & 2.52887900 \\
\hline C & -1.83581700 & 1.25115600 & -1.30103600 \\
\hline $\mathrm{H}$ & -1.29565700 & 3.27827000 & -0.71830200 \\
\hline C & -1.39507500 & -1.02677900 & -2.47882600 \\
\hline $\mathrm{H}$ & -0.31001600 & -0.91916200 & -2.55144000 \\
\hline $\mathrm{H}$ & -1.62547200 & -1.56154900 & -1.54831200 \\
\hline $\mathrm{H}$ & -1.75899600 & -1.62988200 & -3.31682700 \\
\hline C & -3.25660400 & 1.57924900 & 0.85540400 \\
\hline C & -2.21615400 & 1.92114600 & 1.73204700 \\
\hline C & -4.58571200 & 1.77724200 & 1.24466800 \\
\hline C & -2.51186500 & 2.45006600 & 2.97909500 \\
\hline $\mathrm{H}$ & -1.18041600 & 1.76784800 & 1.43092100 \\
\hline C & -4.86991900 & 2.31623500 & 2.49267700 \\
\hline $\mathrm{H}$ & -5.38765400 & 1.51365300 & 0.55896500 \\
\hline
\end{tabular}




$\begin{array}{lrrr}\mathrm{C} & -3.83692200 & 2.65128400 & 3.36205100 \\ \mathrm{H} & -1.70219900 & 2.70904500 & 3.65675400 \\ \mathrm{H} & -5.90449300 & 2.47386400 & 2.78670300 \\ \mathrm{H} & -4.06253000 & 3.07070400 & 4.33938400 \\ \mathrm{C} & -2.96429300 & 1.00230600 & -0.45760400 \\ \mathrm{H} & -1.61545900 & 0.44237500 & -0.36977600\end{array}$

(td)-E-(o) (-1992.815935 a.u.)

\begin{tabular}{|c|c|c|c|}
\hline C & -1.09124000 & -2.84732500 & -0.69328600 \\
\hline C & 0.26844200 & -2.93427300 & -0.50597600 \\
\hline $\mathrm{O}$ & 0.94040400 & -3.71787600 & -1.38714800 \\
\hline C & 2.33736100 & -3.83928200 & -1.18379000 \\
\hline $\mathrm{H}$ & 2.57101900 & -4.15933000 & -0.16247200 \\
\hline $\mathrm{H}$ & 2.85260900 & -2.89021500 & -1.36858900 \\
\hline $\mathrm{H}$ & 2.67872600 & -4.59173100 & -1.89901100 \\
\hline 0 & 0.90825400 & -2.36938400 & 0.45063300 \\
\hline 0 & -3.63742400 & -1.57433000 & 1.67710700 \\
\hline $\mathrm{N}$ & -3.67932000 & -0.69004900 & 0.60371000 \\
\hline C & -4.59285000 & 0.38043000 & 0.81956200 \\
\hline C & -6.35685100 & 2.44821000 & 1.26022500 \\
\hline C & -5.93788700 & 0.08702900 & 1.01437700 \\
\hline C & -4.10621000 & 1.68214600 & 0.87124300 \\
\hline C & -6.82243900 & 1.13594700 & 1.23176500 \\
\hline C & -5.00213000 & 2.72081000 & 1.08504800 \\
\hline $\mathrm{H}$ & -6.27055000 & -0.94720900 & 0.99406000 \\
\hline $\mathrm{H}$ & -3.04012800 & 1.86012000 & 0.74718500 \\
\hline $\mathrm{H}$ & -7.87844600 & 0.92775800 & 1.38078800 \\
\hline $\mathrm{H}$ & -4.63929700 & 3.74408900 & 1.12626700 \\
\hline C & -2.77464700 & -2.66012400 & 1.22494300 \\
\hline $\mathrm{H}$ & -3.41772500 & -3.39396400 & 0.70740100 \\
\hline $\mathrm{H}$ & -7.05390800 & 3.26480500 & 1.42987500 \\
\hline $\mathrm{Rh}$ & 1.76844300 & -0.39811800 & 0.30562400 \\
\hline 0 & 1.83034100 & -0.38568600 & -1.76623200 \\
\hline C & 2.44048900 & 0.57126500 & -2.32478700 \\
\hline 0 & 2.97802600 & 1.57565100 & -1.79666400 \\
\hline $\mathrm{Rh}$ & 2.85604500 & 1.78409000 & 0.25792500 \\
\hline $\mathrm{O}$ & 4.64600100 & 0.79729500 & 0.46156700 \\
\hline C & 4.63125400 & -0.46085400 & 0.45272500 \\
\hline 0 & 3.63964000 & -1.23693800 & 0.36193300 \\
\hline $\mathrm{H}$ & 5.61296100 & -0.95689400 & 0.53311900 \\
\hline 0 & 0.98730000 & 2.64250300 & 0.03743800 \\
\hline C & -0.01082300 & 1.88841000 & 0.10201600 \\
\hline $\mathrm{O}$ & -0.05317800 & 0.63789800 & 0.28117600 \\
\hline $\mathrm{H}$ & -0.99416800 & 2.38425900 & -0.01948100 \\
\hline
\end{tabular}




$\begin{array}{lrrr}\text { O } & 2.64982400 & 1.81581900 & 2.30860800 \\ \mathrm{C} & 2.17379200 & 0.79714300 & 2.87566200 \\ \mathrm{O} & 1.75050300 & -0.27208900 & 2.35562200 \\ \mathrm{H} & 2.11526600 & 0.84211700 & 3.97646200 \\ \mathrm{H} & 2.50522000 & 0.51042400 & -3.42564500 \\ \mathrm{C} & -1.84397700 & -1.97710100 & 0.22843200 \\ \mathrm{H} & -1.58879700 & -3.41735500 & -1.47282300 \\ \mathrm{C} & -2.07489300 & -3.26134100 & 2.40724900 \\ \mathrm{H} & -1.39400800 & -4.03570000 & 2.03797500 \\ \mathrm{H} & -1.47379800 & -2.50185100 & 2.91928000 \\ \mathrm{H} & -2.77737000 & -3.71203500 & 3.11424200 \\ \mathrm{C} & -2.65505900 & -0.18552300 & -1.55691900 \\ \mathrm{C} & -1.41628700 & -0.17147000 & -2.21884700 \\ \mathrm{C} & -3.76366300 & 0.44533000 & -2.15080200 \\ \mathrm{C} & -1.28229100 & 0.51236800 & -3.41903500 \\ \mathrm{H} & -0.56528100 & -0.68869800 & -1.78944200 \\ \mathrm{C} & -3.62265000 & 1.10350900 & -3.36012700 \\ \mathrm{H} & -4.74128800 & 0.40246700 & -1.67708300 \\ \mathrm{C} & -2.37778300 & 1.15130400 & -3.98891800 \\ \mathrm{H} & -0.31308300 & 0.52984900 & -3.91094300 \\ \mathrm{H} & -4.48535500 & 1.58031800 & -3.81776000 \\ \mathrm{H} & -2.26936400 & 1.67943500 & -4.93343400 \\ \mathrm{C} & -2.75334600 & -0.89739700 & -0.29818400 \\ \mathrm{H} & -1.09535500 & -1.40721700 & 0.81657200\end{array}$

TS(td)-VIII-(o) (-1992.769494 a.u.)

$\begin{array}{lrrr}\mathrm{C} & 1.38616500 & 1.98246800 & -0.56196700 \\ \mathrm{C} & 0.11052000 & 2.09158200 & -1.15212700 \\ \mathrm{O} & -0.32773700 & 3.35976700 & -1.22053000 \\ \mathrm{C} & -1.55917100 & 3.56750300 & -1.90948000 \\ \mathrm{H} & -2.39970500 & 3.17808300 & -1.32888700 \\ \mathrm{H} & -1.54391200 & 3.07832200 & -2.88857300 \\ \mathrm{H} & -1.65070400 & 4.64877200 & -2.02523200 \\ \mathrm{O} & -0.55484900 & 1.13877700 & -1.62454300 \\ \mathrm{O} & 2.28514300 & -1.53039000 & 0.15090700 \\ \mathrm{~N} & 3.52958200 & -0.90027600 & 0.31588000 \\ \mathrm{C} & 4.63625300 & -1.69890200 & -0.06314100 \\ \mathrm{C} & 6.79652800 & -3.32480900 & -0.72808100 \\ \mathrm{C} & 4.61813100 & -3.05317600 & 0.27775800 \\ \mathrm{C} & 5.72743600 & -1.15857200 & -0.74671800 \\ \mathrm{C} & 5.69461300 & -3.85871600 & -0.06411300 \\ \mathrm{C} & 6.80587200 & -1.97556700 & -1.06499300 \\ \mathrm{H} & 3.75351100 & -3.45745600 & 0.79550500 \\ \mathrm{H} & 5.73057200 & -0.11024300 & -1.03397500\end{array}$




\begin{tabular}{|c|c|c|c|}
\hline $\mathrm{H}$ & 5.67383800 & -4.91421300 & 0.19639000 \\
\hline $\mathrm{H}$ & 7.65342100 & -1.55127100 & -1.59808400 \\
\hline C & 1.53394900 & -0.69134000 & -0.75525200 \\
\hline $\mathrm{H}$ & 0.49799100 & -0.77409300 & -0.43109100 \\
\hline $\mathrm{H}$ & 7.64014100 & -3.95928600 & -0.98728200 \\
\hline $\mathrm{Rh}$ & -2.16388000 & 0.23437300 & -0.36678000 \\
\hline $\mathrm{O}$ & -3.55826400 & 0.55563100 & -1.84265300 \\
\hline C & -4.72985000 & 0.13139000 & -1.63944300 \\
\hline $\mathrm{O}$ & -5.17744200 & -0.48398600 & -0.63628100 \\
\hline $\mathrm{Rh}$ & -3.87905500 & -0.90514400 & 0.90238100 \\
\hline 0 & -4.32765100 & 0.89641200 & 1.78748900 \\
\hline C & -3.67243300 & 1.90745900 & 1.42816600 \\
\hline $\mathrm{O}$ & -2.74489900 & 1.97723000 & 0.57296600 \\
\hline $\mathrm{H}$ & -3.93829700 & 2.85789800 & 1.91945300 \\
\hline $\mathrm{O}$ & -3.32553100 & -2.63940600 & -0.05655600 \\
\hline C & -2.36782200 & -2.58666600 & -0.87043600 \\
\hline $\mathrm{O}$ & -1.67806900 & -1.58325300 & -1.20660600 \\
\hline $\mathrm{H}$ & -2.09094100 & -3.53781700 & -1.35460700 \\
\hline $\mathrm{O}$ & -2.46594100 & -1.25248300 & 2.36124000 \\
\hline C & -1.31154600 & -0.78482500 & 2.18876400 \\
\hline $\mathrm{O}$ & -0.87312300 & -0.12532600 & 1.20363700 \\
\hline $\mathrm{H}$ & -0.58491900 & -0.97072800 & 2.99647100 \\
\hline $\mathrm{H}$ & -5.45357100 & 0.32788600 & -2.44770400 \\
\hline C & 2.12601600 & 0.69049200 & -0.47453200 \\
\hline $\mathrm{H}$ & 1.89442100 & 2.88136100 & -0.22906400 \\
\hline C & 1.71052900 & -1.13552700 & -2.18867700 \\
\hline $\mathrm{H}$ & 1.10859500 & -0.49513300 & -2.84388700 \\
\hline $\mathrm{H}$ & 2.76625000 & -1.07806100 & -2.48783000 \\
\hline $\mathrm{H}$ & 1.36480200 & -2.16601300 & -2.31200900 \\
\hline C & 4.37740900 & 1.39124600 & 0.59526500 \\
\hline C & 4.58608100 & 2.62039300 & -0.04773700 \\
\hline C & 5.16419000 & 1.07013100 & 1.71294200 \\
\hline C & 5.54883300 & 3.50459600 & 0.41935900 \\
\hline $\mathrm{H}$ & 4.01189800 & 2.87875300 & -0.93554400 \\
\hline C & 6.12042600 & 1.96006400 & 2.17676600 \\
\hline $\mathrm{H}$ & 5.00199200 & 0.12103900 & 2.21845000 \\
\hline C & 6.31709200 & 3.17985800 & 1.53338100 \\
\hline $\mathrm{H}$ & 5.70269000 & 4.45060200 & -0.09374900 \\
\hline $\mathrm{H}$ & 6.71535400 & 1.70178100 & 3.04932000 \\
\hline $\mathrm{H}$ & 7.06818400 & 3.87534800 & 1.89938100 \\
\hline C & 3.37217800 & 0.45062400 & 0.12818000 \\
\hline $\mathrm{H}$ & 2.34191400 & 1.31905500 & -1.46653300 \\
\hline
\end{tabular}

TS(tx)-V (-1992.783111 a.u.) 


\begin{tabular}{|c|c|c|c|}
\hline C & 0.72783800 & -0.05421300 & 1.32097500 \\
\hline C & 0.56401000 & 0.34230500 & 2.74789500 \\
\hline $\mathrm{O}$ & 0.36416800 & 1.65763400 & 2.83809000 \\
\hline C & -0.04534800 & 2.11654500 & 4.12681800 \\
\hline $\mathrm{H}$ & 0.71492300 & 1.88938500 & 4.87979100 \\
\hline $\mathrm{H}$ & -0.98569000 & 1.63228700 & 4.40764900 \\
\hline $\mathrm{H}$ & -0.18227300 & 3.19343800 & 4.03109700 \\
\hline $\mathrm{O}$ & 0.59310500 & -0.43342700 & 3.67432400 \\
\hline $\mathrm{O}$ & 4.10572400 & 0.92192900 & 0.35940000 \\
\hline $\mathrm{N}$ & 3.38192000 & 0.79433100 & -0.85218600 \\
\hline C & 2.75348600 & 2.01384500 & -1.25020400 \\
\hline C & 1.49771400 & 4.35771000 & -2.10972700 \\
\hline C & 2.99210300 & 3.21636600 & -0.58797500 \\
\hline C & 1.91759300 & 1.99439900 & -2.37191200 \\
\hline C & 2.35744900 & 4.37783300 & -1.01808500 \\
\hline C & 1.29122400 & 3.15952900 & -2.78932000 \\
\hline $\mathrm{H}$ & 3.68232300 & 3.23841800 & 0.24963400 \\
\hline $\mathrm{H}$ & 1.76220000 & 1.06794100 & -2.92115400 \\
\hline $\mathrm{H}$ & 2.54461100 & 5.30943800 & -0.48884900 \\
\hline $\mathrm{H}$ & 0.63971100 & 3.12917800 & -3.66025200 \\
\hline C & 3.19970300 & 0.72059400 & 1.45304300 \\
\hline $\mathrm{H}$ & 2.84683000 & 1.69546200 & 1.82890400 \\
\hline $\mathrm{H}$ & 1.00634600 & 5.26923400 & -2.44044900 \\
\hline $\mathrm{Rh}$ & -1.08287200 & -0.30742500 & 0.30812700 \\
\hline $\mathrm{O}$ & -1.45065400 & -2.26001300 & 0.84430100 \\
\hline C & -2.56690300 & -2.75783100 & 0.51113900 \\
\hline $\mathrm{O}$ & -3.50061100 & -2.22763600 & -0.13869600 \\
\hline $\mathrm{Rh}$ & -3.27025100 & -0.28622000 & -0.80980900 \\
\hline $\mathrm{O}$ & -2.33970900 & -0.98175700 & -2.51224000 \\
\hline C & -1.10284400 & -1.16361900 & -2.47219200 \\
\hline $\mathrm{O}$ & -0.31083300 & -0.98594500 & -1.49665600 \\
\hline $\mathrm{H}$ & -0.62718700 & -1.52715500 & -3.39861800 \\
\hline 0 & -4.06996400 & 0.40457700 & 0.95824700 \\
\hline C & -3.30598100 & 0.54189900 & 1.94168300 \\
\hline $\mathrm{O}$ & -2.05855100 & 0.33481000 & 2.00795900 \\
\hline $\mathrm{H}$ & -3.77017800 & 0.88971600 & 2.87998000 \\
\hline $\mathrm{O}$ & -2.84064400 & 1.65347700 & -1.38771700 \\
\hline C & -1.74844700 & 2.15579700 & -1.02591200 \\
\hline 0 & -0.83307000 & 1.63501800 & -0.32356500 \\
\hline $\mathrm{H}$ & -1.54357000 & 3.18758400 & -1.35873200 \\
\hline $\mathrm{H}$ & -2.72911500 & -3.79795700 & 0.83960300 \\
\hline C & 2.01785500 & 0.02007200 & 0.81223600 \\
\hline $\mathrm{H}$ & 1.55311800 & -1.09349800 & 1.33550200 \\
\hline C & 3.90358100 & -0.06985600 & 2.53285200 \\
\hline
\end{tabular}




$\begin{array}{cccc}\mathrm{H} & 3.24352400 & -0.22897500 & 3.39221400 \\ \mathrm{H} & 4.21956600 & -1.04235400 & 2.13871700 \\ \mathrm{H} & 4.79176000 & 0.47252300 & 2.86931200 \\ \mathrm{C} & 3.10126700 & -1.67805700 & -0.69063000 \\ \mathrm{C} & 4.45483100 & -1.84253700 & -0.97385800 \\ \mathrm{C} & 2.29817900 & -2.80027900 & -0.46649100 \\ \mathrm{C} & 5.00144500 & -3.12116800 & -1.02627200 \\ \mathrm{H} & 5.07139900 & -0.96486200 & -1.14861700 \\ \mathrm{C} & 2.85069800 & -4.07504500 & -0.51561300 \\ \mathrm{H} & 1.23033000 & -2.67805400 & -0.27429700 \\ \mathrm{C} & 4.20476400 & -4.23757000 & -0.79336900 \\ \mathrm{H} & 6.05945400 & -3.24371900 & -1.24665500 \\ \mathrm{H} & 2.21866200 & -4.94263500 & -0.34153400 \\ \mathrm{H} & 4.63797900 & -5.23433200 & -0.83070100 \\ \mathrm{C} & 2.46119200 & -0.31086700 & -0.60573900 \\ \mathrm{H} & 1.60824100 & -0.25184300 & -1.28513700\end{array}$

(tx)-D (-1015.137557 a.u.)

\begin{tabular}{|c|c|c|c|}
\hline C & -0.09309400 & -1.67718600 & -1.10716800 \\
\hline C & -0.61713300 & -3.03542400 & -0.90379100 \\
\hline 0 & -0.95962000 & -3.29470600 & 0.37644700 \\
\hline C & -1.50389800 & -4.59322600 & 0.59340100 \\
\hline $\mathrm{H}$ & -0.78921300 & -5.36691600 & 0.29773800 \\
\hline $\mathrm{H}$ & -2.42122100 & -4.72987200 & 0.01306200 \\
\hline $\mathrm{H}$ & -1.71623600 & -4.65779900 & 1.66108600 \\
\hline $\mathrm{O}$ & -0.74549200 & -3.84193100 & -1.79614100 \\
\hline $\mathrm{O}$ & -0.10668800 & 0.69516900 & 1.63818100 \\
\hline$N$ & -0.20881600 & 1.46524500 & 0.46108300 \\
\hline C & -1.55550700 & 1.77377900 & 0.12477300 \\
\hline C & -4.17029300 & 2.49828000 & -0.55431100 \\
\hline C & -2.60890600 & 1.49174500 & 0.99407300 \\
\hline C & -1.81250000 & 2.45096000 & -1.07196100 \\
\hline C & -3.90594100 & 1.85326700 & 0.64777200 \\
\hline C & -3.11287200 & 2.79915200 & -1.40773600 \\
\hline $\mathrm{H}$ & -2.39895900 & 1.00441900 & 1.94067800 \\
\hline $\mathrm{H}$ & -0.98915900 & 2.72730100 & -1.72740900 \\
\hline $\mathrm{H}$ & -4.71923500 & 1.62354800 & 1.33260400 \\
\hline $\mathrm{H}$ & -3.29798300 & 3.32106200 & -2.34375400 \\
\hline C & -0.16920200 & -0.70868200 & 1.28373800 \\
\hline $\mathrm{H}$ & -1.17963300 & -1.09255100 & 1.48879500 \\
\hline $\mathrm{H}$ & -5.18710200 & 2.77537100 & -0.81951200 \\
\hline C & 0.09816500 & -0.71989000 & -0.19323800 \\
\hline $\mathrm{H}$ & 0.12981800 & -1.47093200 & -2.15288900 \\
\hline C & 0.86970300 & -1.42491000 & 2.12105000 \\
\hline
\end{tabular}




$\begin{array}{crrr}\text { H } & 0.82953500 & -2.50135800 & 1.93877100 \\ \mathrm{H} & 1.87020600 & -1.05185100 & 1.87722300 \\ \mathrm{H} & 0.68045900 & -1.23682300 & 3.18248400 \\ \mathrm{C} & 2.04680500 & 0.86980800 & -0.36427700 \\ \mathrm{C} & 2.57134000 & 1.85695400 & 0.46644500 \\ \mathrm{C} & 2.91588900 & 0.04441800 & -1.07826500 \\ \mathrm{C} & 3.94842000 & 2.01905700 & 0.57409700 \\ \mathrm{H} & 1.88865300 & 2.48769300 & 1.02928900 \\ \mathrm{C} & 4.29119500 & 0.20997400 & -0.97279900 \\ \mathrm{H} & 2.51231200 & -0.74032100 & -1.71694100 \\ \mathrm{C} & 4.81152100 & 1.19907300 & -0.14413200 \\ \mathrm{H} & 4.34826600 & 2.79068800 & 1.22796500 \\ \mathrm{H} & 4.95774000 & -0.43859000 & -1.53635000 \\ \mathrm{H} & 5.88777000 & 1.32728000 & -0.05618000 \\ \mathrm{C} & 0.54884300 & 0.68323300 & -0.52069200 \\ \mathrm{H} & 0.23487700 & 0.96237900 & -1.53399200\end{array}$

TS(tx)-VI-(f) (-1015.008033 а.u.)

\begin{tabular}{|c|c|c|c|}
\hline C & -0.98288200 & -1.20193100 & 1.04975000 \\
\hline C & -2.21191800 & -2.00353400 & 1.19071300 \\
\hline $\mathrm{O}$ & -2.91531900 & -2.01226700 & 0.03311500 \\
\hline C & -4.12043600 & -2.76869500 & 0.07240500 \\
\hline $\mathrm{H}$ & -4.80687800 & -2.36116500 & 0.82067800 \\
\hline $\mathrm{H}$ & -3.91654800 & -3.81395100 & 0.32457700 \\
\hline $\mathrm{H}$ & -4.55795500 & -2.69604600 & -0.92423400 \\
\hline 0 & -2.58351200 & -2.55444400 & 2.19989500 \\
\hline 0 & 0.91541400 & -0.27230600 & -1.94557700 \\
\hline N & 1.32867400 & 0.52201000 & -0.83277000 \\
\hline C & 2.48687600 & -0.01484800 & -0.20710200 \\
\hline C & 4.82351700 & -0.92713700 & 1.02379800 \\
\hline C & 3.37373300 & -0.83034800 & -0.90985100 \\
\hline C & 2.77580800 & 0.34900700 & 1.11178300 \\
\hline C & 4.53121200 & -1.28307700 & -0.28847900 \\
\hline C & 3.93998300 & -0.10562500 & 1.71672200 \\
\hline $\mathrm{H}$ & 3.14446700 & -1.09859000 & -1.93624000 \\
\hline $\mathrm{H}$ & 2.08901600 & 0.99309000 & 1.65910900 \\
\hline $\mathrm{H}$ & 5.21369900 & -1.92351900 & -0.84281600 \\
\hline $\mathrm{H}$ & 4.15275000 & 0.18265200 & 2.74361900 \\
\hline C & 0.00524800 & -1.31432400 & -1.50201300 \\
\hline $\mathrm{H}$ & 0.56418100 & -2.25677000 & -1.36439200 \\
\hline $\mathrm{H}$ & 5.73128900 & -1.28505500 & 1.50228200 \\
\hline C & -0.44369800 & -0.76846900 & -0.18999900 \\
\hline $\mathrm{H}$ & -0.27536200 & -1.39461100 & 1.86045900 \\
\hline C & -1.06561400 & -1.44856800 & -2.56005100 \\
\hline
\end{tabular}




$\begin{array}{cccc}\mathrm{H} & -1.74337200 & -2.27306200 & -2.32225400 \\ \mathrm{H} & -1.64422300 & -0.51963500 & -2.61463200 \\ \mathrm{H} & -0.60051700 & -1.62994500 & -3.53397400 \\ \mathrm{C} & -0.64445900 & 1.78977200 & 0.04691400 \\ \mathrm{C} & 0.07678200 & 2.98358800 & 0.09560700 \\ \mathrm{C} & -2.04178100 & 1.83304600 & 0.03784300 \\ \mathrm{C} & -0.58898000 & 4.20270000 & 0.14017300 \\ \mathrm{H} & 1.16392000 & 2.94883100 & 0.07250000 \\ \mathrm{C} & -2.69989100 & 3.05360300 & 0.09122200 \\ \mathrm{H} & -2.61013400 & 0.90625500 & -0.02412900 \\ \mathrm{C} & -1.97822600 & 4.24332700 & 0.14506000 \\ \mathrm{H} & -0.01514900 & 5.12587200 & 0.17139600 \\ \mathrm{H} & -3.78708500 & 3.07591200 & 0.07416500 \\ \mathrm{H} & -2.49853800 & 5.19723500 & 0.17999000 \\ \mathrm{C} & 0.11904400 & 0.50324000 & -0.00266100 \\ \mathrm{H} & -0.36284900 & 0.13020900 & 1.31282400\end{array}$

TS(tx)-VII-(f) (-1015.062294 a.u.)

$\begin{array}{lrrc}\mathrm{C} & 2.46174800 & 0.74429800 & -0.00512200 \\ \mathrm{C} & 3.81731800 & 0.39158800 & -0.26078300 \\ \mathrm{O} & 4.00162100 & -0.95735600 & -0.51239300 \\ \mathrm{C} & 5.34487600 & -1.31924600 & -0.76958600 \\ \mathrm{H} & 5.99341600 & -1.08500600 & 0.08175200 \\ \mathrm{H} & 5.73921900 & -0.79064300 & -1.64426500 \\ \mathrm{H} & 5.34445900 & -2.39636400 & -0.95352500 \\ \mathrm{O} & 4.76851900 & 1.15579800 & -0.26620100 \\ \mathrm{O} & 0.06983600 & -2.06558400 & 0.23031500 \\ \mathrm{~N} & -0.75968700 & -0.93555200 & 0.48080200 \\ \mathrm{C} & -2.06891100 & -1.21591900 & -0.01919400 \\ \mathrm{C} & -4.60510100 & -1.85525700 & -0.92637800 \\ \mathrm{C} & -2.85394700 & -2.10477600 & 0.70863900 \\ \mathrm{C} & -2.53318000 & -0.65431200 & -1.20423300 \\ \mathrm{C} & -4.12279900 & -2.42764900 & 0.24791900 \\ \mathrm{C} & -3.81142600 & -0.97033300 & -1.64865600 \\ \mathrm{H} & -2.45112100 & -2.53392000 & 1.62263500 \\ \mathrm{H} & -1.90058600 & 0.02808200 & -1.76797000 \\ \mathrm{H} & -4.74044900 & -3.12311100 & 0.81016500 \\ \mathrm{H} & -4.18450900 & -0.52854900 & -2.56902400 \\ \mathrm{C} & 1.42510700 & -1.60437700 & 0.38126300 \\ \mathrm{H} & 2.02165800 & -2.20885800 & -0.30412100 \\ \mathrm{H} & -5.60309700 & -2.10123000 & -1.28013300 \\ \mathrm{C} & 1.35620300 & -0.14043100 & -0.03855900 \\ \mathrm{H} & 2.29213400 & 1.80974300 & 0.11122000 \\ \mathrm{C} & 1.89261400 & -1.75405900 & 1.81227700 \\ & & & \\ & & 580 & \\ & & & \\ & & & \\ & & & \\ & & & \end{array}$




$\begin{array}{cccc}\mathrm{H} & 2.94740700 & -1.48213700 & 1.89336200 \\ \mathrm{H} & 1.31425000 & -1.10660300 & 2.48154600 \\ \mathrm{H} & 1.76388400 & -2.79270000 & 2.13207100 \\ \mathrm{C} & -0.65831500 & 1.51159200 & 0.21722000 \\ \mathrm{C} & -1.75287300 & 1.75664200 & 1.05835000 \\ \mathrm{C} & -0.16043600 & 2.55199200 & -0.57951800 \\ \mathrm{C} & -2.32847300 & 3.01772300 & 1.10364200 \\ \mathrm{H} & -2.13566200 & 0.95733000 & 1.68822400 \\ \mathrm{C} & -0.75469000 & 3.80415000 & -0.54021100 \\ \mathrm{H} & 0.68607500 & 2.37148100 & -1.23816800 \\ \mathrm{C} & -1.83577400 & 4.04364900 & 0.30356200 \\ \mathrm{H} & -3.16832100 & 3.19920500 & 1.76940800 \\ \mathrm{H} & -0.36610500 & 4.59996300 & -1.17021100 \\ \mathrm{H} & -2.29130900 & 5.02989500 & 0.33929500 \\ \mathrm{C} & -0.04783700 & 0.18986400 & 0.18178400 \\ \mathrm{H} & 0.75203400 & -0.15140900 & -1.11613300\end{array}$

(tx)-E-(f) (-1015.133182 a.u.)

\begin{tabular}{|c|c|c|c|}
\hline C & 0.51300400 & -1.44973700 & 0.57881400 \\
\hline C & 1.61771100 & -1.19105900 & 1.52593300 \\
\hline 0 & 2.80337100 & -1.04782900 & 0.90206300 \\
\hline C & 3.87457600 & -0.63692600 & 1.74284800 \\
\hline $\mathrm{H}$ & 4.02379900 & -1.34723300 & 2.56103400 \\
\hline H & 3.66665400 & 0.34998400 & 2.17087400 \\
\hline $\mathrm{H}$ & 4.75939500 & -0.59177700 & 1.10717900 \\
\hline O & 1.47865200 & -1.08461500 & 2.72236800 \\
\hline 0 & -1.49781200 & -1.83846400 & -1.14479800 \\
\hline N & -1.15669400 & -0.45200200 & -1.04321600 \\
\hline C & -2.12240300 & 0.19722100 & -0.21442800 \\
\hline C & -3.94187300 & 1.59277200 & 1.37101600 \\
\hline C & -2.99755300 & -0.49976700 & 0.61754100 \\
\hline C & -2.17503300 & 1.59203600 & -0.27121500 \\
\hline C & -3.90648900 & 0.20398100 & 1.40065500 \\
\hline C & -3.07122600 & 2.28280600 & 0.53191500 \\
\hline H & -2.97250800 & -1.58435400 & 0.63229700 \\
\hline $\mathrm{H}$ & -1.51306500 & 2.12155900 & -0.95265100 \\
\hline $\mathrm{H}$ & -4.59068400 & -0.34450500 & 2.04415500 \\
\hline H & -3.10118200 & 3.36890700 & 0.48679300 \\
\hline C & -0.32020300 & -2.49117700 & -1.59978800 \\
\hline $\mathrm{H}$ & -0.19222900 & -2.31574400 & -2.68421100 \\
\hline $\mathrm{H}$ & -4.65260000 & 2.13606400 & 1.98881400 \\
\hline C & 0.78692300 & -1.76379900 & -0.87407000 \\
\hline $\mathrm{H}$ & -0.36393700 & -1.88898000 & 1.05137600 \\
\hline C & -0.47019700 & -3.96136400 & -1.30376900 \\
\hline
\end{tabular}




$\begin{array}{crrc}\mathrm{H} & 0.39664800 & -4.51422300 & -1.67939800 \\ \mathrm{H} & -0.54709400 & -4.12659100 & -0.22358600 \\ \mathrm{H} & -1.36903500 & -4.36121800 & -1.78257900 \\ \mathrm{C} & 0.91522800 & 0.86576500 & -0.66017100 \\ \mathrm{C} & 1.14132800 & 1.67901400 & 0.44899200 \\ \mathrm{C} & 1.26635100 & 1.32066400 & -1.93107100 \\ \mathrm{C} & 1.73184100 & 2.92786600 & 0.29032000 \\ \mathrm{H} & 0.83861300 & 1.33315600 & 1.43703100 \\ \mathrm{C} & 1.84858400 & 2.57125400 & -2.09092900 \\ \mathrm{H} & 1.06215800 & 0.68518900 & -2.79208700 \\ \mathrm{C} & 2.08430600 & 3.37551800 & -0.97860100 \\ \mathrm{H} & 1.90574000 & 3.55736200 & 1.15970100 \\ \mathrm{H} & 2.11986200 & 2.92121100 & -3.08411300 \\ \mathrm{H} & 2.54071100 & 4.35473900 & -1.10288800 \\ \mathrm{C} & 0.22389600 & -0.44168700 & -0.52971800 \\ \mathrm{H} & 1.81929000 & -1.86254700 & -1.19793600\end{array}$

TS(tx)-VIII-(f) (-1015.051331 a.u.)

$\begin{array}{lrrc}\mathrm{C} & 2.49571300 & 0.73633200 & -0.05733900 \\ \mathrm{C} & 3.84896900 & 0.33755300 & -0.27833200 \\ \mathrm{O} & 3.98645500 & -1.00590000 & -0.54524900 \\ \mathrm{C} & 5.32297700 & -1.41688100 & -0.78001900 \\ \mathrm{H} & 5.95714300 & -1.21987200 & 0.09057100 \\ \mathrm{H} & 5.75375200 & -0.88940200 & -1.63751600 \\ \mathrm{H} & 5.28288000 & -2.48938300 & -0.98086400 \\ \mathrm{O} & 4.81400100 & 1.08203500 & -0.24414900 \\ \mathrm{O} & 0.02815300 & -2.07058600 & 0.27935500 \\ \mathrm{~N} & -0.79683600 & -0.92007200 & 0.39735700 \\ \mathrm{C} & -2.10555300 & -1.21098600 & -0.06021200 \\ \mathrm{C} & -4.69353300 & -1.84860500 & -0.85612600 \\ \mathrm{C} & -2.76903900 & -2.28878800 & 0.52879800 \\ \mathrm{C} & -2.72853800 & -0.46032800 & -1.05717900 \\ \mathrm{C} & -4.05550800 & -2.60816400 & 0.12178800 \\ \mathrm{C} & -4.02654700 & -0.77712300 & -1.43903100 \\ \mathrm{H} & -2.25774600 & -2.86590800 & 1.29406800 \\ \mathrm{H} & -2.20099900 & 0.36276400 & -1.53107500 \\ \mathrm{H} & -4.56764100 & -3.45162600 & 0.57815100 \\ \mathrm{H} & -4.51195600 & -0.18767100 & -2.21299600 \\ \mathrm{C} & 1.38074100 & -1.60970700 & 0.41460900 \\ \mathrm{H} & 1.98386800 & -2.24586700 & -0.23825700 \\ \mathrm{H} & -5.70610000 & -2.09492400 & -1.16508300 \\ \mathrm{C} & 1.30835900 & -0.16867100 & -0.05730600 \\ \mathrm{H} & 2.31954400 & 1.80525500 & 0.00371700 \\ \mathrm{C} & 1.84671600 & -1.70713300 & 1.85374300\end{array}$




$\begin{array}{cccc}\mathrm{H} & 2.90249100 & -1.43522000 & 1.93470500 \\ \mathrm{H} & 1.26210600 & -1.03589500 & 2.49389100 \\ \mathrm{H} & 1.71838900 & -2.73224800 & 2.21386000 \\ \mathrm{C} & -0.62040000 & 1.52763900 & 0.18029500 \\ \mathrm{C} & -1.67965500 & 1.79352900 & 1.06360900 \\ \mathrm{C} & -0.13072800 & 2.57287300 & -0.61692600 \\ \mathrm{C} & -2.22232300 & 3.06572100 & 1.14716200 \\ \mathrm{H} & -2.05859300 & 0.99401700 & 1.69564300 \\ \mathrm{C} & -0.68773200 & 3.84167500 & -0.53553100 \\ \mathrm{H} & 0.67495600 & 2.38725400 & -1.32385900 \\ \mathrm{C} & -1.73198000 & 4.09508800 & 0.34765400 \\ \mathrm{H} & -3.03381500 & 3.25679600 & 1.84501200 \\ \mathrm{H} & -0.30123400 & 4.63697300 & -1.16788400 \\ \mathrm{H} & -2.16104600 & 5.09149000 & 0.41506600 \\ \mathrm{C} & -0.04356600 & 0.19524100 & 0.10465300 \\ \mathrm{H} & 1.64273200 & 0.09399500 & -1.15415500\end{array}$

TS(tx)-VI-(c) (-1992.709962 a.u.)

$\begin{array}{lccc}\mathrm{C} & 1.26960000 & -1.45714300 & -0.32087200 \\ \mathrm{C} & 1.18912800 & -2.93057200 & -0.21776300 \\ \mathrm{O} & 1.45193100 & -3.34569700 & 1.03434200 \\ \mathrm{C} & 1.37247400 & -4.75327100 & 1.23036000 \\ \mathrm{H} & 2.08181000 & -5.27408300 & 0.58008500 \\ \mathrm{H} & 0.36356900 & -5.11570600 & 1.01073600 \\ \mathrm{H} & 1.61866400 & -4.92626900 & 2.27918900 \\ \mathrm{O} & 0.94923500 & -3.67004700 & -1.14146300 \\ \mathrm{O} & 2.45237000 & 1.00362200 & 2.18152500 \\ \mathrm{~N} & 2.75032400 & 1.51120600 & 0.87991000 \\ \mathrm{C} & 1.93818700 & 2.61370100 & 0.52678100 \\ \mathrm{C} & 0.42025100 & 4.85599900 & -0.17182900 \\ \mathrm{C} & 1.53240600 & 3.51879900 & 1.51100700 \\ \mathrm{C} & 1.60550900 & 2.84707200 & -0.80931800 \\ \mathrm{C} & 0.77431500 & 4.62719700 & 1.15734100 \\ \mathrm{C} & 0.84783200 & 3.96246700 & -1.14890800 \\ \mathrm{H} & 1.80644800 & 3.33017800 & 2.54450700 \\ \mathrm{H} & 1.92028100 & 2.14509600 & -1.57953300 \\ \mathrm{H} & 0.45958800 & 5.32476500 & 1.93135900 \\ \mathrm{H} & 0.58577400 & 4.12783100 & -2.19216500 \\ \mathrm{C} & 1.65494800 & -0.20482400 & 2.08579500 \\ \mathrm{H} & 0.59892700 & 0.02625800 & 2.28614800 \\ \mathrm{H} & -0.17409800 & 5.72498700 & -0.44218800 \\ \mathrm{Rh} & -1.11946800 & -0.48956900 & -0.15624900 \\ \mathrm{O} & -1.70826900 & -2.12696500 & -1.24384900 \\ \mathrm{C} & -2.93068100 & -2.23904400 & -1.53546900\end{array}$




\begin{tabular}{|c|c|c|c|}
\hline $\mathrm{O}$ & -3.87146900 & -1.45026000 & -1.25360900 \\
\hline $\mathrm{Rh}$ & -3.40678800 & 0.26039500 & -0.21954400 \\
\hline $\mathrm{O}$ & -2.95873300 & 1.22494800 & -1.98010500 \\
\hline C & -1.77528100 & 1.17384900 & -2.40173400 \\
\hline $\mathrm{O}$ & -0.79239400 & 0.56048700 & -1.89458800 \\
\hline $\mathrm{H}$ & -1.56349100 & 1.72860900 & -3.32949400 \\
\hline $\mathrm{O}$ & -3.69200600 & -0.74385900 & 1.55183300 \\
\hline C & -2.72433500 & -1.38379700 & 2.03859500 \\
\hline $\mathrm{O}$ & -1.54827200 & -1.48981500 & 1.58608100 \\
\hline $\mathrm{H}$ & -2.92254700 & -1.92094400 & 2.97983200 \\
\hline $\mathrm{O}$ & -2.85081900 & 1.94915700 & 0.79242400 \\
\hline C & -1.63970000 & 2.04795000 & 1.12446400 \\
\hline $\mathrm{O}$ & -0.71490300 & 1.21045000 & 0.92861900 \\
\hline $\mathrm{H}$ & -1.34013200 & 2.97012000 & 1.64776200 \\
\hline $\mathrm{H}$ & -3.20333700 & -3.14034400 & -2.10660300 \\
\hline C & 1.85537300 & -0.58164400 & 0.65325300 \\
\hline $\mathrm{H}$ & 0.73029200 & -1.07732600 & -1.20501400 \\
\hline C & 2.20032800 & -1.17844900 & 3.10686800 \\
\hline $\mathrm{H}$ & 1.54421200 & -2.04904300 & 3.18663800 \\
\hline $\mathrm{H}$ & 3.20488800 & -1.51385400 & 2.82560500 \\
\hline $\mathrm{H}$ & 2.25612700 & -0.67718100 & 4.07862000 \\
\hline C & 4.02462900 & 0.00859300 & -0.62368800 \\
\hline C & 5.14910400 & -0.04576700 & 0.20961000 \\
\hline C & 4.17733000 & -0.20355600 & -1.99138200 \\
\hline C & 6.39397300 & -0.34751900 & -0.32079700 \\
\hline $\mathrm{H}$ & 5.02593600 & 0.15558000 & 1.27238200 \\
\hline C & 5.42796900 & -0.51349600 & -2.52150200 \\
\hline $\mathrm{H}$ & 3.31514000 & -0.11444800 & -2.65243600 \\
\hline C & 6.53514300 & -0.58831400 & -1.68750100 \\
\hline $\mathrm{H}$ & 7.26064700 & -0.39908500 & 0.33420600 \\
\hline $\mathrm{H}$ & 5.53343000 & -0.68236200 & -3.59029500 \\
\hline $\mathrm{H}$ & 7.51330200 & -0.82418100 & -2.09997900 \\
\hline C & 2.70921400 & 0.33602700 & 0.03284800 \\
\hline $\mathrm{H}$ & 2.03417000 & -0.44590200 & -1.05470000 \\
\hline
\end{tabular}

TS(tx)-VII-(c) (-1992.797315 a.u.)

$\begin{array}{lrrr}\mathrm{C} & 0.08987400 & 1.72998600 & 0.81300800 \\ \mathrm{C} & -0.71662500 & 2.95763300 & 0.70371800 \\ \mathrm{O} & -0.87681700 & 3.38628400 & -0.57090700 \\ \mathrm{C} & -1.76629500 & 4.48519900 & -0.71621100 \\ \mathrm{H} & -1.40579900 & 5.35900800 & -0.16273800 \\ \mathrm{H} & -2.75986800 & 4.22008200 & -0.34259600 \\ \mathrm{H} & -1.80712700 & 4.70224700 & -1.78492200 \\ \mathrm{O} & -1.22696200 & 3.51364200 & 1.64960800 \\ & & \end{array}$




\begin{tabular}{|c|c|c|c|}
\hline $\mathrm{O}$ & 2.59910300 & 1.34219600 & -1.87894600 \\
\hline$N$ & 3.33479500 & 0.86171100 & -0.74872300 \\
\hline C & 4.08601600 & -0.28377500 & -1.16668900 \\
\hline C & 5.58931500 & -2.42938500 & -2.06025000 \\
\hline C & 3.61973100 & -1.57892700 & -0.95965600 \\
\hline C & 5.28946500 & -0.05082400 & -1.82399600 \\
\hline C & 4.38398700 & -2.65248900 & -1.40108200 \\
\hline C & 6.03856800 & -1.12913500 & -2.27708400 \\
\hline $\mathrm{H}$ & 2.67065200 & -1.73925400 & -0.45091900 \\
\hline $\mathrm{H}$ & 5.61566800 & 0.97557400 & -1.97453400 \\
\hline $\mathrm{H}$ & 4.03197400 & -3.66783600 & -1.23504200 \\
\hline $\mathrm{H}$ & 6.97889900 & -0.95540600 & -2.79392900 \\
\hline C & 1.57875400 & 2.19397400 & -1.33974800 \\
\hline $\mathrm{H}$ & 0.74861100 & 2.17306900 & -2.05042400 \\
\hline $\mathrm{H}$ & 6.18136400 & -3.27284800 & -2.40678800 \\
\hline $\mathrm{Rh}$ & -1.36388200 & 0.13900900 & 0.16265100 \\
\hline $\mathrm{O}$ & -1.57567700 & -0.44277300 & 2.12899800 \\
\hline C & -2.36159700 & -1.40381100 & 2.37946300 \\
\hline $\mathrm{O}$ & -3.04823600 & -2.08629500 & 1.58114400 \\
\hline $\mathrm{Rh}$ & -2.97028900 & -1.62804100 & -0.43363300 \\
\hline $\mathrm{O}$ & -1.35329900 & -2.90556900 & -0.67607600 \\
\hline C & -0.20462100 & -2.46617700 & -0.47113000 \\
\hline $\mathrm{O}$ & 0.13607400 & -1.29848300 & -0.09417000 \\
\hline $\mathrm{H}$ & 0.63147100 & -3.17024800 & -0.62947600 \\
\hline $\mathrm{O}$ & -4.46169900 & -0.24163900 & -0.16027300 \\
\hline C & -4.12961600 & 0.92942200 & 0.14974300 \\
\hline $\mathrm{O}$ & -2.97180400 & 1.40095700 & 0.33612600 \\
\hline $\mathrm{H}$ & -4.95297000 & 1.65170000 & 0.27790300 \\
\hline $\mathrm{O}$ & -2.75565700 & -1.01189800 & -2.39481700 \\
\hline C & -1.94618700 & -0.08780600 & -2.65138900 \\
\hline $\mathrm{O}$ & -1.21957300 & 0.57452700 & -1.85393400 \\
\hline $\mathrm{H}$ & -1.84777900 & 0.19010200 & -3.71462900 \\
\hline $\mathrm{H}$ & -2.44458900 & -1.67280100 & 3.44626800 \\
\hline C & 1.24059900 & 1.50495000 & -0.03120100 \\
\hline $\mathrm{H}$ & 0.24503000 & 1.48502600 & 1.86611500 \\
\hline C & 2.12892500 & 3.58931900 & -1.10508000 \\
\hline $\mathrm{H}$ & 1.35455800 & 4.26340300 & -0.73359800 \\
\hline $\mathrm{H}$ & 2.95546200 & 3.56414900 & -0.38563800 \\
\hline $\mathrm{H}$ & 2.51148200 & 3.98200700 & -2.05194100 \\
\hline C & 2.82311700 & 0.21837900 & 1.59029200 \\
\hline C & 4.15751200 & 0.36564800 & 1.99139600 \\
\hline C & 1.90985200 & -0.41959800 & 2.43785700 \\
\hline C & 4.56757900 & -0.10889700 & 3.22861500 \\
\hline $\mathrm{H}$ & 4.86384600 & 0.86785300 & 1.33447900 \\
\hline
\end{tabular}




$\begin{array}{cccc}\mathrm{C} & 2.33586500 & -0.89758800 & 3.67092600 \\ \mathrm{H} & 0.87685300 & -0.56969500 & 2.13509800 \\ \mathrm{C} & 3.65865300 & -0.74287000 & 4.07096500 \\ \mathrm{H} & 5.60244300 & 0.01897800 & 3.53569300 \\ \mathrm{H} & 1.62070400 & -1.39356800 & 4.32213500 \\ \mathrm{H} & 3.98254900 & -1.11600700 & 5.03943800 \\ \mathrm{C} & 2.42324300 & 0.77972300 & 0.29320200 \\ \mathrm{H} & 1.30037300 & 0.21362800 & -0.29272000\end{array}$

(tx)-E-(c) (-1992.827867 a.u.)

\begin{tabular}{|c|c|c|c|}
\hline C & 0.22709500 & 1.10564500 & 0.96790700 \\
\hline C & -0.27779300 & 2.40352600 & 1.43144300 \\
\hline $\mathrm{O}$ & -0.41041600 & 3.31047500 & 0.42063500 \\
\hline C & -1.01912100 & 4.53145700 & 0.80681800 \\
\hline $\mathrm{H}$ & -0.41015600 & 5.06018500 & 1.55085200 \\
\hline $\mathrm{H}$ & -2.00684100 & 4.34752600 & 1.24042600 \\
\hline $\mathrm{H}$ & -1.10898100 & 5.12995000 & -0.10178000 \\
\hline $\mathrm{O}$ & -0.57826200 & 2.67633700 & 2.57417400 \\
\hline $\mathrm{O}$ & 3.15099600 & 1.79366300 & -1.29637200 \\
\hline $\mathrm{N}$ & 3.43896200 & 0.59634000 & -0.67021700 \\
\hline C & 4.69685600 & 0.04389200 & -1.03871000 \\
\hline C & 7.14511500 & -1.00298300 & -1.74007900 \\
\hline C & 5.81358600 & 0.87380800 & -1.02209500 \\
\hline C & 4.77335200 & -1.29241000 & -1.41869900 \\
\hline C & 7.04488700 & 0.33723000 & -1.37487900 \\
\hline C & 6.01283600 & -1.81375000 & -1.76338000 \\
\hline $\mathrm{H}$ & 5.70239500 & 1.91681900 & -0.73690800 \\
\hline $\mathrm{H}$ & 3.87474200 & -1.90406200 & -1.44015000 \\
\hline $\mathrm{H}$ & 7.92917200 & 0.96854700 & -1.36467300 \\
\hline $\mathrm{H}$ & 6.09198400 & -2.85513700 & -2.06274400 \\
\hline C & 2.07331600 & 2.36435900 & -0.47141200 \\
\hline $\mathrm{H}$ & 1.49798400 & 2.99859900 & -1.14751800 \\
\hline $\mathrm{H}$ & 8.11147600 & -1.41693300 & -2.01592200 \\
\hline $\mathrm{Rh}$ & -1.51374600 & 0.10082900 & 0.12716000 \\
\hline $\mathrm{O}$ & -1.61550600 & -1.21525900 & 1.72220100 \\
\hline C & -2.55357100 & -2.06709200 & 1.72199700 \\
\hline $\mathrm{O}$ & -3.44418000 & -2.26486300 & 0.86294200 \\
\hline $\mathrm{Rh}$ & -3.49775700 & -1.05335900 & -0.82354000 \\
\hline $\mathrm{O}$ & -2.16711100 & -2.32193100 & -1.79552500 \\
\hline C & -0.94067700 & -2.13675300 & -1.65309200 \\
\hline $\mathrm{O}$ & -0.34001000 & -1.26337500 & -0.95212600 \\
\hline $\mathrm{H}$ & -0.27524800 & -2.82268700 & -2.20870200 \\
\hline $\mathrm{O}$ & -4.65477000 & 0.30506800 & 0.21174100 \\
\hline C & -4.07155200 & 1.18263200 & 0.89360900 \\
\hline
\end{tabular}




$\begin{array}{lrrr}\mathrm{O} & -2.83238700 & 1.37095400 & 1.06045400 \\ \mathrm{H} & -4.72241200 & 1.89655600 & 1.42759200 \\ \mathrm{O} & -3.36137500 & 0.27899400 & -2.40729600 \\ \mathrm{C} & -2.42757100 & 1.11561000 & -2.41410600 \\ \mathrm{O} & -1.51197400 & 1.30018900 & -1.55904900 \\ \mathrm{H} & -2.38847900 & 1.79130400 & -3.28719200 \\ \mathrm{H} & -2.57694500 & -2.72886300 & 2.60684000 \\ \mathrm{C} & 1.29890400 & 1.09314400 & -0.10379100 \\ \mathrm{H} & 0.49729300 & 0.52087400 & 1.85201700 \\ \mathrm{C} & 2.70626300 & 3.13421400 & 0.66402100 \\ \mathrm{H} & 1.94639000 & 3.63178900 & 1.27226300 \\ \mathrm{H} & 3.28216500 & 2.47347300 & 1.32717100 \\ \mathrm{H} & 3.38057700 & 3.89804300 & 0.26392500 \\ \mathrm{C} & 2.65809500 & -0.89057400 & 1.08009600 \\ \mathrm{C} & 3.88173400 & -0.98872600 & 1.76737100 \\ \mathrm{C} & 1.59722600 & -1.73820400 & 1.42598600 \\ \mathrm{C} & 4.03945400 & -1.93109100 & 2.76869200 \\ \mathrm{H} & 4.69403300 & -0.30134800 & 1.53930700 \\ \mathrm{C} & 1.77407300 & -2.69063900 & 2.42008300 \\ \mathrm{H} & 0.64741100 & -1.65619200 & 0.90854200 \\ \mathrm{C} & 2.98808300 & -2.78928100 & 3.09074500 \\ \mathrm{H} & 4.98006500 & -1.99023200 & 3.30970300 \\ \mathrm{H} & 0.94398800 & -3.34211900 & 2.68050200 \\ \mathrm{H} & 3.11518100 & -3.52696500 & 3.87944000 \\ \mathrm{C} & 2.47346500 & 0.17259600 & 0.10603700 \\ \mathrm{H} & 0.87471300 & 0.73866800 & -1.06601000\end{array}$

TS(tx)-VIII-(c) (-1992.76773 a.u.)

$\begin{array}{lrrr}\text { C } & 0.53631500 & 1.96767900 & 0.79360500 \\ \mathrm{C} & -0.29040100 & 3.16381900 & 0.54838900 \\ \mathrm{O} & -0.42289400 & 3.45483200 & -0.75993900 \\ \mathrm{C} & -1.31740400 & 4.52420200 & -1.04561500 \\ \mathrm{H} & -0.97751500 & 5.45186500 & -0.57316400 \\ \mathrm{H} & -2.31926100 & 4.28361100 & -0.67837200 \\ \mathrm{H} & -1.32306600 & 4.62930700 & -2.13141900 \\ \mathrm{O} & -0.83540300 & 3.78497600 & 1.43222700 \\ \mathrm{O} & 3.29328600 & 1.55335000 & -1.75062100 \\ \mathrm{~N} & 3.41554600 & 0.38939200 & -0.97769700 \\ \mathrm{C} & 2.93853500 & -0.76692700 & -1.70429600 \\ \mathrm{C} & 1.97543600 & -2.97583500 & -3.12403700 \\ \mathrm{C} & 2.64593900 & -0.65257300 & -3.06303000 \\ \mathrm{C} & 2.77390300 & -1.99594600 & -1.06116500 \\ \mathrm{C} & 2.16562400 & -1.75554800 & -3.76187100 \\ \mathrm{C} & 2.28953800 & -3.08663100 & -1.77277800\end{array}$




\begin{tabular}{|c|c|c|c|}
\hline $\mathrm{H}$ & 2.79212700 & 0.29860500 & -3.56213200 \\
\hline $\mathrm{H}$ & 3.00563700 & -2.10202300 & -0.00520200 \\
\hline $\mathrm{H}$ & 1.93940100 & -1.65093600 & -4.82098700 \\
\hline $\mathrm{H}$ & 2.15809900 & -4.03689100 & -1.25938200 \\
\hline C & 2.09453300 & 2.26118500 & -1.35782300 \\
\hline $\mathrm{H}$ & 1.30475800 & 2.13690900 & -2.11332200 \\
\hline $\mathrm{H}$ & 1.59574200 & -3.83371700 & -3.67266400 \\
\hline $\mathrm{Rh}$ & -1.06012800 & 0.30036000 & 0.30428200 \\
\hline $\mathrm{O}$ & -1.63102300 & 0.40396200 & 2.27156900 \\
\hline C & -2.62434800 & -0.29604600 & 2.62891100 \\
\hline $\mathrm{O}$ & -3.29546800 & -1.10955400 & 1.94735000 \\
\hline $\mathrm{Rh}$ & -2.73776600 & -1.45618900 & -0.01189700 \\
\hline $\mathrm{O}$ & -1.33022400 & -2.82635500 & 0.62465800 \\
\hline C & -0.17470100 & -2.40249400 & 0.85500500 \\
\hline $\mathrm{O}$ & 0.25472100 & -1.21260200 & 0.78861900 \\
\hline $\mathrm{H}$ & 0.57650500 & -3.15108000 & 1.16116300 \\
\hline $\mathrm{O}$ & -4.03965200 & 0.00849300 & -0.63083300 \\
\hline C & -3.65526100 & 1.20235400 & -0.56672800 \\
\hline $\mathrm{O}$ & -2.53064300 & 1.65143700 & -0.20448000 \\
\hline $\mathrm{H}$ & -4.39133700 & 1.96754900 & -0.86567000 \\
\hline $\mathrm{O}$ & -2.01969100 & -1.67222400 & -1.93655500 \\
\hline C & -1.11735600 & -0.89424400 & -2.32943800 \\
\hline $\mathrm{O}$ & -0.56855000 & 0.05850900 & -1.69869300 \\
\hline $\mathrm{H}$ & -0.74620900 & -1.04713400 & -3.35654400 \\
\hline $\mathrm{H}$ & -2.93114600 & -0.16817200 & 3.67993300 \\
\hline C & 1.72475400 & 1.60411500 & -0.03898200 \\
\hline $\mathrm{H}$ & 0.62324000 & 1.74743000 & 1.85600500 \\
\hline C & 2.53193100 & 3.70488300 & -1.21861100 \\
\hline $\mathrm{H}$ & 1.68933400 & 4.37540200 & -1.04352500 \\
\hline $\mathrm{H}$ & 3.25106900 & 3.79459500 & -0.39646800 \\
\hline $\mathrm{H}$ & 3.03496500 & 4.00942700 & -2.14211500 \\
\hline C & 3.14414700 & -0.01394300 & 1.44122000 \\
\hline C & 4.46372400 & -0.50599100 & 1.48650000 \\
\hline C & 2.34165200 & -0.14283700 & 2.58983400 \\
\hline C & 4.96097100 & -1.09843600 & 2.63620100 \\
\hline $\mathrm{H}$ & 5.09028700 & -0.39555400 & 0.60507100 \\
\hline C & 2.84846700 & -0.73934200 & 3.73423900 \\
\hline $\mathrm{H}$ & 1.30065600 & 0.15812900 & 2.57878900 \\
\hline C & 4.15587400 & -1.21745800 & 3.76584000 \\
\hline $\mathrm{H}$ & 5.98418500 & -1.46526600 & 2.65132800 \\
\hline $\mathrm{H}$ & 2.20919500 & -0.83940500 & 4.60779000 \\
\hline $\mathrm{H}$ & 4.54431700 & -1.68450500 & 4.66759300 \\
\hline C & 2.72170300 & 0.65814200 & 0.22895900 \\
\hline $\mathrm{H}$ & 0.78829600 & 0.79709200 & -0.15254800 \\
\hline
\end{tabular}


TS(tx)-VI-(o) (-1992.718392 a.u.)

\begin{tabular}{|c|c|c|c|}
\hline C & 1.68259700 & 0.24074500 & -2.49143100 \\
\hline C & 0.83656000 & -0.92839700 & -2.31259200 \\
\hline $\mathrm{O}$ & 1.47715000 & -2.01440300 & -1.86453900 \\
\hline C & 0.66666100 & -3.16303500 & -1.59410000 \\
\hline $\mathrm{H}$ & 0.01432500 & -3.38213800 & -2.44298300 \\
\hline $\mathrm{H}$ & 0.05409900 & -2.99057400 & -0.70413900 \\
\hline $\mathrm{H}$ & 1.36818600 & -3.98277600 & -1.42587800 \\
\hline $\mathrm{O}$ & -0.36191600 & -0.96176900 & -2.62477400 \\
\hline $\mathrm{O}$ & 4.57588000 & -0.87761200 & -0.94461000 \\
\hline$N$ & 3.98468800 & -0.09871600 & 0.11415200 \\
\hline C & 3.65298900 & -0.97732900 & 1.19776100 \\
\hline C & 2.83993000 & -2.52828600 & 3.35263200 \\
\hline C & 3.87653900 & -0.52587800 & 2.49428400 \\
\hline C & 3.05335500 & -2.21545300 & 0.96844500 \\
\hline C & 3.45403300 & -1.29858200 & 3.57289900 \\
\hline C & 2.65168100 & -2.98892800 & 2.04926300 \\
\hline $\mathrm{H}$ & 4.35970500 & 0.43790900 & 2.63742100 \\
\hline $\mathrm{H}$ & 2.91413000 & -2.54268200 & -0.05685500 \\
\hline $\mathrm{H}$ & 3.61585000 & -0.94308000 & 4.58757200 \\
\hline $\mathrm{H}$ & 2.17893200 & -3.95343300 & 1.87362600 \\
\hline C & 4.38288400 & -0.13598700 & -2.16372600 \\
\hline $\mathrm{H}$ & 4.44778300 & -0.87994900 & -2.96638200 \\
\hline $\mathrm{H}$ & 2.51770100 & -3.13428300 & 4.19611800 \\
\hline $\mathrm{Rh}$ & -1.65697800 & -0.34602500 & -0.86050300 \\
\hline 0 & -0.89353500 & 1.55662500 & -0.94100300 \\
\hline C & -1.27405000 & 2.38316900 & -0.06561400 \\
\hline $\mathrm{O}$ & -2.09283400 & 2.20070400 & 0.87464300 \\
\hline $\mathrm{Rh}$ & -2.98061500 & 0.35365900 & 1.02817100 \\
\hline 0 & -1.45036200 & -0.28728200 & 2.26020800 \\
\hline C & -0.42807400 & -0.77920200 & 1.72074900 \\
\hline O & -0.18981600 & -0.91915900 & 0.48592900 \\
\hline $\mathrm{H}$ & 0.36895800 & -1.13759800 & 2.39929500 \\
\hline $\mathrm{O}$ & -4.42116500 & 0.94334800 & -0.30647700 \\
\hline C & -4.20544700 & 0.75321000 & -1.53411900 \\
\hline 0 & -3.18995100 & 0.24329500 & -2.08118000 \\
\hline $\mathrm{H}$ & -5.00662300 & 1.07304100 & -2.21978300 \\
\hline $\mathrm{O}$ & -3.79214000 & -1.53603100 & 1.06243900 \\
\hline C & -3.37719600 & -2.37462900 & 0.21959900 \\
\hline $\mathrm{O}$ & -2.50492000 & -2.21679700 & -0.67917300 \\
\hline $\mathrm{H}$ & -3.83114500 & -3.37786200 & 0.27377200 \\
\hline $\mathrm{H}$ & -0.82422700 & 3.38770800 & -0.13341800 \\
\hline C & 2.98589100 & 0.34222300 & -1.90284500 \\
\hline
\end{tabular}




$\begin{array}{lrrr}\mathrm{H} & 1.38965800 & 0.79958500 & -3.38507400 \\ \mathrm{C} & 5.41201900 & 0.96543400 & -2.31763000 \\ \mathrm{H} & 5.24087700 & 1.52711500 & -3.24306600 \\ \mathrm{H} & 5.35339200 & 1.65933600 & -1.47127400 \\ \mathrm{H} & 6.42144300 & 0.54326200 & -2.34645200 \\ \mathrm{C} & 2.35185700 & 1.79945400 & 0.12134800 \\ \mathrm{C} & 2.48271700 & 3.02728500 & -0.53184500 \\ \mathrm{C} & 1.75769700 & 1.76100100 & 1.38561100 \\ \mathrm{C} & 2.02978300 & 4.19472200 & 0.07316100 \\ \mathrm{H} & 2.94418400 & 3.06245500 & -1.51665100 \\ \mathrm{C} & 1.31647700 & 2.92942100 & 1.99456200 \\ \mathrm{H} & 1.64618800 & 0.80741300 & 1.89536300 \\ \mathrm{C} & 1.45029600 & 4.15027200 & 1.33833600 \\ \mathrm{H} & 2.14020400 & 5.14510400 & -0.44380700 \\ \mathrm{H} & 0.84797200 & 2.88008000 & 2.97446400 \\ \mathrm{H} & 1.10006400 & 5.06460100 & 1.81133900 \\ \mathrm{C} & 2.85653600 & 0.53932100 & -0.51927200 \\ \mathrm{H} & 1.55170800 & 0.21360700 & -0.90191500\end{array}$

TS(tx)-VII-(o) (-1992.782447 a.u.)

$\begin{array}{lrrc}\text { C } & 1.02564100 & 1.12230000 & -0.83961400 \\ \mathrm{C} & 0.13967100 & 2.17968700 & -1.07570700 \\ \mathrm{O} & 0.67992800 & 3.42387500 & -1.11239700 \\ \mathrm{C} & -0.24810400 & 4.49478100 & -1.24436600 \\ \mathrm{H} & -0.99266100 & 4.47150500 & -0.44183900 \\ \mathrm{H} & -0.77376600 & 4.44293600 & -2.20252400 \\ \mathrm{H} & 0.34365500 & 5.41068600 & -1.18958600 \\ \mathrm{O} & -1.09473800 & 2.06755600 & -1.25860800 \\ \mathrm{O} & 4.33285800 & 2.05877400 & 0.50845000 \\ \mathrm{~N} & 4.35554300 & 0.63521000 & 0.54829400 \\ \mathrm{C} & 5.69903600 & 0.18354100 & 0.37890700 \\ \mathrm{C} & 8.33257700 & -0.63617600 & 0.14074200 \\ \mathrm{C} & 6.58003200 & 0.39067500 & 1.43681800 \\ \mathrm{C} & 6.12385500 & -0.41690500 & -0.80257800 \\ \mathrm{C} & 7.90131900 & -0.01537400 & 1.31105600 \\ \mathrm{C} & 7.44471300 & -0.83704100 & -0.91120100 \\ \mathrm{H} & 6.21458900 & 0.87462600 & 2.33912100 \\ \mathrm{H} & 5.42642900 & -0.55948400 & -1.62545200 \\ \mathrm{H} & 8.59580300 & 0.14588300 & 2.13151600 \\ \mathrm{H} & 7.78216900 & -1.31497100 & -1.82734000 \\ \mathrm{C} & 2.95454100 & 2.42879000 & 0.32374400 \\ \mathrm{H} & 2.97132400 & 3.36313200 & -0.24204800 \\ \mathrm{H} & 9.36527100 & -0.96307700 & 0.04842800 \\ \mathrm{Rh} & -2.28057800 & 0.48063000 & -0.35388900 \\ & & & \\ & & 590 & \\ & & & \\ & & & \end{array}$




$\begin{array}{lccc}\text { O } & -1.21767800 & 0.61105500 & 1.41262200 \\ \mathrm{C} & -1.62498700 & -0.07453000 & 2.39100900 \\ \mathrm{O} & -2.60231200 & -0.86473400 & 2.44430800 \\ \mathrm{Rh} & -3.74008200 & -1.11656200 & 0.74193200 \\ \mathrm{O} & -2.47268400 & -2.62119000 & 0.12733900 \\ \mathrm{C} & -1.47658300 & -2.29487000 & -0.56550500 \\ \mathrm{O} & -1.13319800 & -1.14454700 & -0.95610800 \\ \mathrm{H} & -0.80803500 & -3.11856000 & -0.87388500 \\ \mathrm{O} & -4.91572200 & 0.47704300 & 1.29803300 \\ \mathrm{C} & -4.54387500 & 1.63379000 & 0.96856400 \\ \mathrm{O} & -3.52105300 & 1.97442800 & 0.31289200 \\ \mathrm{H} & -5.19522700 & 2.46182600 & 1.29491400 \\ \mathrm{O} & -4.77519100 & -1.25661700 & -1.03126000 \\ \mathrm{C} & -4.39638300 & -0.53937900 & -1.99511800 \\ \mathrm{O} & -3.43167700 & 0.27193400 & -2.04192800 \\ \mathrm{H} & -4.98406000 & -0.63096800 & -2.92394300 \\ \mathrm{H} & -1.04225200 & 0.03541300 & 3.32191100 \\ \mathrm{C} & 2.37795000 & 1.26353200 & -0.46334100 \\ \mathrm{H} & 0.60076000 & 0.12935300 & -0.92606800 \\ \mathrm{C} & 2.24536300 & 2.58318500 & 1.65303500 \\ \mathrm{H} & 1.21369400 & 2.91213800 & 1.50227800 \\ \mathrm{H} & 2.21898300 & 1.62769800 & 2.19001000 \\ \mathrm{H} & 2.77366700 & 3.31866400 & 2.26734600 \\ \mathrm{C} & 3.04928000 & -1.24649200 & -0.39018700 \\ \mathrm{C} & 3.49726700 & -2.18031700 & 0.55313400 \\ \mathrm{C} & 2.33861200 & -1.68611800 & -1.51437200 \\ \mathrm{C} & 3.22566500 & -3.52998100 & 0.37707900 \\ \mathrm{H} & 4.03857200 & -1.83938900 & 1.43259500 \\ \mathrm{C} & 2.08769900 & -3.03951600 & -1.69007100 \\ \mathrm{H} & 1.98424800 & -0.97011900 & -2.25247500 \\ \mathrm{C} & 2.52329300 & -3.96364100 & -0.74363600 \\ \mathrm{H} & 3.56378700 & -4.24637700 & 1.12124700 \\ \mathrm{H} & 1.54239200 & -3.37094300 & -2.57050300 \\ \mathrm{H} & 2.31452900 & -5.02187000 & -0.87948800 \\ \mathrm{H} & 0.17772400 & -0.16889000 \\ & 3.29042600 & -1.39796500\end{array}$

(tx)-E-(o) (-1992.809979 a.u.)

$\begin{array}{lrrr}\text { C } & 1.10573300 & 2.15803400 & 0.31069700 \\ \mathrm{C} & 0.16739300 & 2.51946800 & -0.62405100 \\ \mathrm{O} & 0.65989400 & 3.04998400 & -1.80146800 \\ \mathrm{C} & -0.30626100 & 3.26507600 & -2.81723800 \\ \mathrm{H} & -1.05711600 & 3.99988200 & -2.51069500 \\ \mathrm{H} & -0.82871400 & 2.33313000 & -3.07094200 \\ & & \end{array}$




\begin{tabular}{|c|c|c|c|}
\hline $\mathrm{H}$ & 0.24698800 & 3.63663300 & -3.68397100 \\
\hline $\mathrm{O}$ & -1.09197700 & 2.42345400 & -0.52472300 \\
\hline $\mathrm{O}$ & 4.72785200 & 1.88934100 & 0.91460400 \\
\hline$N$ & 4.34093300 & 0.70526900 & 0.29869600 \\
\hline C & 5.36730300 & -0.28431800 & 0.32578000 \\
\hline C & 7.34628800 & -2.19620900 & 0.41822400 \\
\hline C & 5.88567200 & -0.65759300 & 1.56124300 \\
\hline C & 5.83275500 & -0.82794200 & -0.86629200 \\
\hline C & 6.88067500 & -1.62584900 & 1.60026900 \\
\hline C & 6.82625600 & -1.79675100 & -0.81039800 \\
\hline $\mathrm{H}$ & 5.50574600 & -0.18837000 & 2.46543900 \\
\hline $\mathrm{H}$ & 5.41383600 & -0.49957300 & -1.81406800 \\
\hline $\mathrm{H}$ & 7.29366500 & -1.93511200 & 2.55648800 \\
\hline $\mathrm{H}$ & 7.20054900 & -2.23540300 & -1.73123800 \\
\hline C & 3.48404300 & 2.62522900 & 1.13337200 \\
\hline $\mathrm{H}$ & 3.75163200 & 3.67599900 & 0.98972100 \\
\hline $\mathrm{H}$ & 8.12485200 & -2.95378300 & 0.45415700 \\
\hline $\mathrm{Rh}$ & -2.05638500 & 0.59364100 & 0.05038000 \\
\hline $\mathrm{O}$ & -2.26010700 & 1.16235300 & 2.01442500 \\
\hline C & -2.94630900 & 0.42010100 & 2.76935500 \\
\hline $\mathrm{O}$ & -3.52764800 & -0.66277100 & 2.49567000 \\
\hline $\mathrm{Rh}$ & -3.37893200 & -1.38568500 & 0.57148400 \\
\hline $\mathrm{O}$ & -1.63573400 & -2.36428200 & 1.07260500 \\
\hline C & -0.56203600 & -1.71898800 & 0.96731200 \\
\hline $\mathrm{O}$ & -0.38276900 & -0.53159900 & 0.58248200 \\
\hline $\mathrm{H}$ & 0.35659700 & -2.26752300 & 1.24739200 \\
\hline $\mathrm{O}$ & -5.04416500 & -0.29776800 & 0.04200500 \\
\hline C & -4.88166700 & 0.89705300 & -0.32197600 \\
\hline $\mathrm{O}$ & -3.80875800 & 1.55118800 & -0.42385100 \\
\hline $\mathrm{H}$ & -5.79889900 & 1.44868300 & -0.58955900 \\
\hline $\mathrm{O}$ & -3.12292000 & -1.96161600 & -1.39815300 \\
\hline C & -2.47725300 & -1.19893000 & -2.16024600 \\
\hline $\mathrm{O}$ & -1.92208700 & -0.09620200 & -1.89731900 \\
\hline $\mathrm{H}$ & -2.37812300 & -1.54135600 & -3.20733700 \\
\hline $\mathrm{H}$ & -3.04674000 & 0.77013800 & 3.81108600 \\
\hline C & 2.55258200 & 2.12685700 & 0.01420000 \\
\hline $\mathrm{H}$ & 0.73171100 & 1.65881800 & 1.19920600 \\
\hline C & 3.00848300 & 2.35383000 & 2.53823400 \\
\hline $\mathrm{H}$ & 2.09357000 & 2.91844000 & 2.73775500 \\
\hline $\mathrm{H}$ & 2.77839000 & 1.28873800 & 2.67646300 \\
\hline $\mathrm{H}$ & 3.77703500 & 2.64501300 & 3.26065100 \\
\hline C & 2.50232100 & -0.36132600 & -0.90562100 \\
\hline C & 2.71025700 & -1.69617800 & -0.52390000 \\
\hline C & 1.60667900 & -0.06431500 & -1.94274700 \\
\hline
\end{tabular}




$\begin{array}{lccc}\mathrm{C} & 2.04183600 & -2.71534100 & -1.18354500 \\ \mathrm{H} & 3.36952700 & -1.93279800 & 0.30844700 \\ \mathrm{C} & 0.96892500 & -1.09478100 & -2.61735500 \\ \mathrm{H} & 1.41895100 & 0.96998400 & -2.22382700 \\ \mathrm{C} & 1.17723500 & -2.41629500 & -2.23659800 \\ \mathrm{H} & 2.18786800 & -3.74651700 & -0.87247100 \\ \mathrm{H} & 0.27899100 & -0.85228100 & -3.42060900 \\ \mathrm{H} & 0.65252800 & -3.21909300 & -2.74973800 \\ \mathrm{C} & 3.15058600 & 0.74557500 & -0.22992300 \\ \mathrm{H} & 2.74826800 & 2.69631900 & -0.91008600\end{array}$

TS(tx)-VIII-(o) (-1992.7576 a.u.)

\begin{tabular}{lrrc} 
C & 0.87803100 & -1.97910200 & 1.03619600 \\
$\mathrm{C}$ & 0.23868300 & -1.38508600 & 2.15261100 \\
$\mathrm{O}$ & 1.02460400 & -1.05461700 & 3.20265400 \\
$\mathrm{C}$ & 0.36535500 & -0.34468500 & 4.25082400 \\
$\mathrm{H}$ & -0.37382500 & -0.98053200 & 4.74549900 \\
$\mathrm{H}$ & -0.14863900 & 0.54001500 & 3.85443800 \\
$\mathrm{H}$ & 1.14947400 & -0.05598000 & 4.95307300 \\
$\mathrm{O}$ & -0.99435700 & -1.23735200 & 2.24118000 \\
$\mathrm{O}$ & 4.16472500 & -2.20252900 & -0.64780200 \\
$\mathrm{~N}$ & 4.09206300 & -0.81670300 & -0.41089100 \\
$\mathrm{C}$ & 5.34884300 & -0.18763000 & -0.44075700 \\
$\mathrm{C}$ & 7.84270900 & 1.04047600 & -0.57956700 \\
$\mathrm{C}$ & 6.29626200 & -0.64024000 & -1.36262500 \\
$\mathrm{C}$ & 5.64882400 & 0.87184500 & 0.41991700 \\
$\mathrm{C}$ & 7.54028800 & -0.02768800 & -1.41898500 \\
$\mathrm{C}$ & 6.89137800 & 1.48462400 & 0.33450900 \\
$\mathrm{H}$ & 6.04786500 & -1.47061300 & -2.01580000 \\
$\mathrm{H}$ & 4.91704900 & 1.20923500 & 1.14851900 \\
$\mathrm{H}$ & 8.27784900 & -0.38695100 & -2.13256300 \\
$\mathrm{H}$ & 7.12145200 & 2.30946600 & 1.00454800 \\
$\mathrm{C}$ & 2.81896100 & -2.67986900 & -0.49185500 \\
$\mathrm{H}$ & 2.90598300 & -3.70514500 & -0.10897600 \\
$\mathrm{H}$ & 8.81595800 & 1.52130300 & -0.63321000 \\
$\mathrm{Rh}$ & -2.06347700 & -0.42276800 & 0.49240700 \\
$\mathrm{O}$ & -2.25174500 & -2.21662500 & -0.50819000 \\
$\mathrm{C}$ & -2.94585600 & -2.22171000 & -1.56386900 \\
$\mathrm{O}$ & -3.53401300 & -1.25846100 & -2.11914700 \\
$\mathrm{Rh}$ & -3.36637200 & 0.60870000 & -1.26591700 \\
$\mathrm{O}$ & -1.62528000 & 0.93696600 & -2.30893000 \\
$\mathrm{C}$ & -0.54972000 & 0.57653400 & -1.76533000 \\
$\mathrm{O}$ & -0.38468200 & 0.00621800 & -0.65194100 \\
$\mathrm{H}$ & 0.37485100 & 0.78578400 & -2.33230200 \\
& & & \\
& 593 & \\
\hline
\end{tabular}




\begin{tabular}{lrrr} 
O & -5.03537000 & 0.22940700 & -0.12595100 \\
$\mathrm{C}$ & -4.88281400 & -0.36939900 & 0.97145000 \\
$\mathrm{O}$ & -3.81232300 & -0.77574400 & 1.50133500 \\
$\mathrm{H}$ & -5.80216000 & -0.56584300 & 1.54734600 \\
$\mathrm{O}$ & -3.09131400 & 2.40581900 & -0.29469400 \\
$\mathrm{C}$ & -2.44452600 & 2.40326300 & 0.78437400 \\
$\mathrm{O}$ & -1.92139900 & 1.42484000 & 1.38680500 \\
$\mathrm{H}$ & -2.31691000 & 3.38509100 & 1.27250300 \\
$\mathrm{H}$ & -3.04587800 & -3.20063800 & -2.06203600 \\
$\mathrm{C}$ & 2.24122900 & -1.70548700 & 0.51794100 \\
$\mathrm{H}$ & 0.22518900 & -2.59069100 & 0.41242000 \\
$\mathrm{C}$ & 2.08108700 & -2.64381100 & -1.81654700 \\
$\mathrm{H}$ & 1.05170500 & -3.00318400 & -1.70408600 \\
$\mathrm{H}$ & 2.03609600 & -1.61330600 & -2.19154400 \\
$\mathrm{H}$ & 2.58949900 & -3.26714200 & -2.55851300 \\
$\mathrm{C}$ & 2.47682000 & 0.86867700 & 0.32946400 \\
$\mathrm{C}$ & 2.64262600 & 1.68712300 & -0.80268500 \\
$\mathrm{C}$ & 1.76610400 & 1.36368000 & 1.42484800 \\
$\mathrm{C}$ & 2.06792100 & 2.94717800 & -0.84257900 \\
$\mathrm{H}$ & 3.19258900 & 1.30798500 & -1.66239800 \\
$\mathrm{C}$ & 1.19905300 & 2.63221800 & 1.37971300 \\
$\mathrm{H}$ & 1.68287300 & 0.75753200 & 2.31802800 \\
$\mathrm{C}$ & 1.33469800 & 3.42272800 & 0.24536000 \\
$\mathrm{H}$ & 2.18309100 & 3.56130300 & -1.73261400 \\
$\mathrm{H}$ & 0.64011000 & 2.99520000 & 2.23952700 \\
$\mathrm{H}$ & 0.87918900 & 4.40938100 & 0.20575200 \\
$\mathrm{C}$ & 2.94800100 & -0.50848900 & 0.25956900 \\
$\mathrm{H}$ & 2.16083900 & -2.28659100 & 1.58213500 \\
\hline & & & \\
\hline & & & \\
& & &
\end{tabular}

TS(cd)-V (-1992.776657 a.u.)

$\begin{array}{lccc}\text { C } & 0.62525700 & -0.07066100 & 0.53434100 \\ \text { C } & 0.84614100 & -0.09272900 & 2.01145600 \\ \text { O } & 1.27292500 & -1.30557500 & 2.38977800 \\ \text { C } & 1.38856800 & -1.49244100 & 3.80071500 \\ \text { H } & 0.40858200 & -1.37508900 & 4.27278500 \\ \text { H } & 2.08283200 & -0.76395700 & 4.23066500 \\ \text { H } & 1.76176600 & -2.50727200 & 3.93784000 \\ \text { O } & 0.64972700 & 0.83590000 & 2.75524600 \\ \text { N } & 3.99581400 & 0.13177700 & -0.71247000 \\ \text { C } & 4.36477000 & -1.22575400 & -0.48349900 \\ \text { C } & 5.20881500 & -3.86305800 & -0.04899100 \\ \text { C } & 4.70542900 & -1.65839900 & 0.80353400 \\ \text { C } & 4.48392600 & -2.11498900 & -1.55503700 \\ \text { C } & 5.11598900 & -2.96952700 & 1.01205400 \\ & & & \end{array}$




\begin{tabular}{|c|c|c|c|}
\hline C & 4.89833400 & -3.42186300 & -1.33113700 \\
\hline $\mathrm{H}$ & 4.67593900 & -0.97421100 & 1.64827200 \\
\hline $\mathrm{H}$ & 4.25104900 & -1.76792100 & -2.55626900 \\
\hline $\mathrm{H}$ & 5.36918500 & -3.28923700 & 2.02035000 \\
\hline $\mathrm{H}$ & 4.97931400 & -4.10176300 & -2.17618500 \\
\hline C & 1.89188400 & 0.18124200 & -1.74632700 \\
\hline $\mathrm{H}$ & 1.43058200 & 1.09248300 & -2.15458700 \\
\hline $\mathrm{H}$ & 5.52859700 & -4.88779800 & 0.12000300 \\
\hline $\mathrm{Rh}$ & -1.39024400 & -0.17853800 & 0.04374600 \\
\hline $\mathrm{O}$ & -1.35631400 & -2.04317400 & -0.84339500 \\
\hline C & -2.44863200 & -2.50364800 & -1.29247600 \\
\hline $\mathrm{O}$ & -3.58574900 & -1.97690600 & -1.27613800 \\
\hline $\mathrm{Rh}$ & -3.80931100 & -0.12991900 & -0.37717000 \\
\hline $\mathrm{O}$ & -3.38268400 & 0.81056900 & -2.16577500 \\
\hline C & -2.18466900 & 1.03402800 & -2.45427100 \\
\hline $\mathrm{O}$ & -1.14241700 & 0.74207700 & -1.79266400 \\
\hline $\mathrm{H}$ & -1.99908600 & 1.55033800 & -3.41117600 \\
\hline O & -4.03881800 & -1.05858500 & 1.45282500 \\
\hline C & -3.01673900 & -1.29748500 & 2.13766200 \\
\hline $\mathrm{O}$ & -1.80314400 & -1.06443200 & 1.85709100 \\
\hline $\mathrm{H}$ & -3.18354300 & -1.77629600 & 3.11702800 \\
\hline $\mathrm{O}$ & -3.85941800 & 1.70564800 & 0.55570500 \\
\hline C & -2.77145400 & 2.18979100 & 0.95195600 \\
\hline $\mathrm{O}$ & -1.61072700 & 1.69206700 & 0.87796900 \\
\hline $\mathrm{H}$ & -2.82113800 & 3.18244000 & 1.42934200 \\
\hline $\mathrm{H}$ & -2.37606500 & -3.50000500 & -1.75996200 \\
\hline C & 1.74461100 & 0.21687600 & -0.24391400 \\
\hline $\mathrm{H}$ & 1.44628400 & -1.01523900 & 0.09011300 \\
\hline C & 1.35166500 & -1.04680000 & -2.44478800 \\
\hline $\mathrm{H}$ & 1.73948000 & -1.96572700 & -1.98886200 \\
\hline $\mathrm{H}$ & 0.26062100 & -1.06205900 & -2.40076000 \\
\hline $\mathrm{H}$ & 1.66538300 & -1.02659200 & -3.49286500 \\
\hline C & 3.07645300 & 2.25379500 & 0.16298300 \\
\hline C & 4.11875300 & 2.92167800 & -0.47816200 \\
\hline C & 2.02660900 & 2.98382800 & 0.72499500 \\
\hline C & 4.11817300 & 4.30983900 & -0.54534200 \\
\hline $\mathrm{H}$ & 4.92066100 & 2.34253400 & -0.92813900 \\
\hline C & 2.03174900 & 4.37289800 & 0.65066200 \\
\hline $\mathrm{H}$ & 1.20501000 & 2.48428600 & 1.23750700 \\
\hline C & 3.07467200 & 5.03901000 & 0.01658300 \\
\hline $\mathrm{H}$ & 4.93562400 & 4.82315700 & -1.04647300 \\
\hline $\mathrm{H}$ & 1.21086100 & 4.93265800 & 1.09233600 \\
\hline $\mathrm{H}$ & 3.07343700 & 6.12498000 & -0.04296200 \\
\hline $\mathrm{O}$ & 3.32439300 & 0.30731000 & -1.94767900 \\
\hline
\end{tabular}




$\begin{array}{llll}\mathrm{C} & 3.08229000 & 0.73473400 & 0.24777900 \\ \mathrm{H} & 3.30613100 & 0.39703800 & 1.26500600\end{array}$

(cd)-D (-1015.136951 a.u.)

\begin{tabular}{|c|c|c|c|}
\hline C & -0.39966400 & 1.74294400 & 1.16413000 \\
\hline C & -0.70924800 & 2.67396100 & 0.07080900 \\
\hline 0 & -0.64832500 & 3.94269100 & 0.51169200 \\
\hline C & -0.91297500 & 4.93512100 & -0.47372600 \\
\hline $\mathrm{H}$ & -1.91450600 & 4.80553700 & -0.89523900 \\
\hline $\mathrm{H}$ & -0.18496600 & 4.87587000 & -1.28846400 \\
\hline $\mathrm{H}$ & -0.83567700 & 5.89587400 & 0.03598700 \\
\hline O & -0.97486400 & 2.38841600 & -1.07962900 \\
\hline N & 0.68476900 & -1.38676000 & -0.02647600 \\
\hline C & 1.99910200 & -1.02507500 & -0.42307400 \\
\hline C & 4.58934100 & -0.41915800 & -1.30486100 \\
\hline C & 2.20943300 & -0.51819400 & -1.71080200 \\
\hline C & 3.09927600 & -1.26158400 & 0.40396600 \\
\hline C & 3.49385600 & -0.21130100 & -2.13701600 \\
\hline C & 4.38017300 & -0.95393000 & -0.03948000 \\
\hline $\mathrm{H}$ & 1.37064800 & -0.38106600 & -2.38880800 \\
\hline $\mathrm{H}$ & 2.94012100 & -1.69578200 & 1.38513200 \\
\hline H & 3.63622200 & 0.18984200 & -3.13789700 \\
\hline $\mathrm{H}$ & 5.22512200 & -1.13960100 & 0.62012600 \\
\hline C & 0.12417800 & -0.54497800 & 2.07682300 \\
\hline H & -0.77693300 & -0.88044700 & 2.61788700 \\
\hline $\mathrm{H}$ & 5.59330800 & -0.17865400 & -1.64440500 \\
\hline C & -0.27912600 & 0.42170400 & 1.00399900 \\
\hline $\mathrm{H}$ & -0.22447700 & 2.19748600 & 2.13738700 \\
\hline C & 1.16102900 & -0.03831600 & 3.05531700 \\
\hline $\mathrm{H}$ & 2.02287300 & 0.38047400 & 2.52525600 \\
\hline $\mathrm{H}$ & 0.73506800 & 0.73770200 & 3.70164900 \\
\hline $\mathrm{H}$ & 1.50248800 & -0.85815500 & 3.69471200 \\
\hline C & -1.74499000 & -1.06009300 & -0.38385600 \\
\hline C & -1.91681300 & -2.43068500 & -0.20404600 \\
\hline C & -2.85078800 & -0.26259400 & -0.68525400 \\
\hline C & -3.18181300 & -2.99687900 & -0.32625100 \\
\hline $\mathrm{H}$ & -1.05319400 & -3.04794200 & 0.02696400 \\
\hline C & -4.11178900 & -0.83230500 & -0.80691700 \\
\hline $\mathrm{H}$ & -2.70856000 & 0.80473300 & -0.84390400 \\
\hline C & -4.28184200 & -2.20148400 & -0.62498000 \\
\hline $\mathrm{H}$ & -3.30568100 & -4.06839900 & -0.18615100 \\
\hline $\mathrm{H}$ & -4.96543000 & -0.20360600 & -1.05009600 \\
\hline $\mathrm{H}$ & -5.26950000 & -2.64702300 & -0.71956200 \\
\hline 0 & 0.60659300 & -1.69806600 & 1.35603200 \\
\hline
\end{tabular}




$\begin{array}{llcc}\mathrm{C} & -0.38119400 & -0.40788900 & -0.25097000 \\ \mathrm{H} & -0.16442700 & 0.20056600 & -1.13288400\end{array}$

\begin{tabular}{|c|c|c|c|}
\hline \multicolumn{4}{|c|}{ TS(cd)-VI-(f) (-1015.012662 a.u.) } \\
\hline $\mathrm{C}$ & -1.93312600 & -0.59649600 & 1.14495700 \\
\hline $\mathrm{C}$ & -3.40522100 & -0.57842200 & 1.03680000 \\
\hline 0 & -3.79370100 & -0.59461800 & -0.26003200 \\
\hline $\mathrm{C}$ & -5.20233500 & -0.57868200 & -0.45822700 \\
\hline $\mathrm{H}$ & -5.64106600 & 0.32698000 & -0.02843700 \\
\hline $\mathrm{H}$ & -5.67398300 & -1.44647700 & 0.01375500 \\
\hline $\mathrm{H}$ & -5.35937600 & -0.60032300 & -1.53732200 \\
\hline 0 & -4.17959500 & -0.53517200 & 1.96403700 \\
\hline 0 & 0.58272200 & -1.97251000 & -0.77956300 \\
\hline $\mathrm{N}$ & 1.11394600 & -0.68111300 & -0.47594900 \\
\hline $\mathrm{C}$ & 2.43858400 & -0.75806200 & -0.01997300 \\
\hline $\mathrm{C}$ & 5.11074800 & -0.84903400 & 0.78635400 \\
\hline $\mathrm{C}$ & 3.27162000 & -1.78365500 & -0.47413500 \\
\hline $\mathrm{C}$ & 2.94372800 & 0.21961300 & 0.84284400 \\
\hline $\mathrm{C}$ & 4.59597100 & -1.82569400 & -0.06010900 \\
\hline $\mathrm{C}$ & 4.27633300 & 0.17196600 & 1.22896800 \\
\hline $\mathrm{H}$ & 2.86919300 & -2.53940200 & -1.14048000 \\
\hline $\mathrm{H}$ & 2.29703300 & 1.01352600 & $1.2074640 \mathrm{C}$ \\
\hline $\mathrm{H}$ & 5.23441400 & -2.63323300 & -0.41139000 \\
\hline $\mathrm{H}$ & 4.65980600 & 0.93899400 & $1.8979490 \mathrm{C}$ \\
\hline C & -0.81911100 & -1.77170000 & -1.04355500 \\
\hline $\mathrm{H}$ & 6.15038400 & -0.88511300 & 1.10103700 \\
\hline $\mathrm{C}$ & -1.08447400 & -0.75898700 & 0.02340000 \\
\hline $\mathrm{H}$ & -1.60841600 & -0.96794400 & 2.11858600 \\
\hline $\mathrm{C}$ & 0.06730100 & 1.52301700 & -0.09284200 \\
\hline $\mathrm{C}$ & 0.30835700 & 1.94453100 & -1.40532900 \\
\hline $\mathrm{C}$ & -0.15735900 & 2.48239900 & 0.89266500 \\
\hline $\mathrm{C}$ & 0.29522800 & 3.29557100 & -1.72220700 \\
\hline $\mathrm{H}$ & 0.51153200 & 1.19877500 & -2.17058900 \\
\hline $\mathrm{C}$ & -0.18027200 & 3.83569000 & 0.57162200 \\
\hline $\mathrm{H}$ & -0.30855000 & 2.16800200 & 1.92427100 \\
\hline C & 0.04563800 & 4.24605800 & -0.73608100 \\
\hline $\mathrm{H}$ & 0.47758200 & 3.60806300 & -2.74774700 \\
\hline $\mathrm{H}$ & -0.36092200 & 4.56989500 & 1.35284800 \\
\hline $\mathrm{H}$ & 0.03788200 & 5.30411700 & -0.98635300 \\
\hline $\mathrm{C}$ & 0.06853300 & 0.03865800 & 0.18534900 \\
\hline $\mathrm{H}$ & -0.69400300 & 0.21080300 & 1.44376600 \\
\hline $\mathrm{C}$ & -1.51409900 & -3.09989100 & -0.92740900 \\
\hline $\mathrm{H}$ & -2.58448900 & -2.96861700 & -1.11215000 \\
\hline $\mathrm{H}$ & -1.11669200 & -3.81343600 & -1.65481400 \\
\hline
\end{tabular}




$\begin{array}{rrrr}H & -1.37658100 & -3.50819200 & 0.07921000 \\ H & -0.94303000 & -1.33150100 & -2.05204300\end{array}$

\begin{tabular}{|c|c|c|c|}
\hline \multicolumn{4}{|c|}{ TS(cd)-VII-(f) (-1015.058668 a.u.) } \\
\hline $\mathrm{C}$ & -1.89135500 & -1.66640700 & -0.78285600 \\
\hline $\mathrm{C}$ & -2.57940600 & -1.35828200 & 0.42617300 \\
\hline 0 & -3.75479500 & -2.02528400 & 0.55596700 \\
\hline $\mathrm{C}$ & -4.43408800 & -1.79438300 & 1.77721900 \\
\hline $\mathrm{H}$ & -4.67408000 & -0.73319600 & 1.90428400 \\
\hline $\mathrm{H}$ & -3.82899400 & -2.10822900 & 2.63495300 \\
\hline $\mathrm{H}$ & -5.35209300 & -2.38344700 & 1.73194300 \\
\hline 0 & -2.16255300 & -0.59621200 & 1.29973500 \\
\hline $\mathrm{N}$ & 1.36210200 & 0.07790100 & -1.10913300 \\
\hline $\mathrm{C}$ & 2.31642300 & 0.04030200 & -0.04958300 \\
\hline $\mathrm{C}$ & 4.23864600 & -0.02802900 & 1.96284700 \\
\hline $\mathrm{C}$ & 1.94620500 & 0.29336200 & 1.27245700 \\
\hline $\mathrm{C}$ & 3.64618700 & -0.24498900 & -0.36710700 \\
\hline $\mathrm{C}$ & 2.91633000 & 0.26379800 & 2.27017900 \\
\hline $\mathrm{C}$ & 4.59646200 & -0.28664000 & 0.64088500 \\
\hline $\mathrm{H}$ & 0.91082100 & 0.51321900 & 1.52907700 \\
\hline $\mathrm{H}$ & 3.90874600 & -0.44773700 & -1.40103200 \\
\hline $\mathrm{H}$ & 2.62340400 & 0.46042600 & 3.29843900 \\
\hline $\mathrm{H}$ & 5.62899200 & -0.51911900 & 0.39162000 \\
\hline $\mathrm{C}$ & 0.48902800 & -1.97247300 & -1.64580800 \\
\hline $\mathrm{H}$ & 0.08904300 & -2.35053200 & -2.59661200 \\
\hline $\mathrm{H}$ & 4.98955600 & -0.05583000 & 2.74810600 \\
\hline $\mathrm{C}$ & -0.58885900 & -1.14284900 & -0.96363000 \\
\hline $\mathrm{H}$ & -2.18869100 & -2.54139200 & -1.35406900 \\
\hline $\mathrm{C}$ & 1.01592400 & -3.10566000 & -0.79091000 \\
\hline $\mathrm{H}$ & 1.43302200 & -2.72070500 & 0.14746500 \\
\hline $\mathrm{H}$ & 0.20472500 & -3.80015500 & -0.54640000 \\
\hline $\mathrm{H}$ & 1.80325000 & -3.65249700 & -1.31772300 \\
\hline $\mathrm{C}$ & -0.61004500 & 1.47408300 & -0.56576400 \\
\hline $\mathrm{C}$ & 0.19977400 & 2.61634300 & -0.43067500 \\
\hline $\mathrm{C}$ & -2.00423900 & 1.62351200 & -0.53856400 \\
\hline C & -0.37181500 & 3.86309000 & -0.24881000 \\
\hline $\mathrm{H}$ & 1.28037600 & 2.51774500 & -0.49307600 \\
\hline $\mathrm{C}$ & -2.56744300 & 2.88023800 & -0.37016000 \\
\hline $\mathrm{H}$ & -2.63838200 & 0.75900200 & -0.69133200 \\
\hline $\mathrm{C}$ & -1.75860400 & 3.99867200 & -0.20774100 \\
\hline $\mathrm{H}$ & 0.26659800 & 4.73671300 & -0.14450200 \\
\hline $\mathrm{H}$ & -3.64953800 & 2.98256200 & -0.35923100 \\
\hline $\mathrm{H}$ & -2.20561800 & 4.97854700 & -0.05798900 \\
\hline 0 & 1.51431000 & -1.02954100 & -1.99321900 \\
\hline
\end{tabular}




$\begin{array}{lrrr}\mathrm{C} & 0.00212000 & 0.19190000 & -0.84629400 \\ \mathrm{H} & -0.27903600 & -0.84592200 & 0.18899400\end{array}$

(cd)-E-(f) (-1015.134466 a.u.)

\begin{tabular}{|c|c|c|c|}
\hline C & -0.66243700 & -1.39690500 & -0.77096400 \\
\hline C & -1.88543900 & -0.98106100 & -1.48756000 \\
\hline $\mathrm{O}$ & -1.60063600 & -0.62813200 & -2.75565400 \\
\hline C & -2.71011200 & -0.12354900 & -3.49127400 \\
\hline $\mathrm{H}$ & -3.11218900 & 0.77429400 & -3.00920300 \\
\hline $\mathrm{H}$ & -3.50921500 & -0.86822700 & -3.55419500 \\
\hline $\mathrm{H}$ & -2.33199100 & 0.11511800 & -4.48586500 \\
\hline $\mathrm{O}$ & -2.99587900 & -0.92471100 & -1.01160000 \\
\hline$N$ & 1.13467600 & -0.72332900 & 0.87923400 \\
\hline C & 2.06923900 & 0.03228000 & 0.10908300 \\
\hline C & 3.83466300 & 1.63382600 & -1.33800100 \\
\hline C & 2.17527600 & 1.39547000 & 0.39664400 \\
\hline C & 2.86637900 & -0.53344600 & -0.88526800 \\
\hline C & 3.04293800 & 2.19140400 & -0.33759900 \\
\hline C & 3.74900400 & 0.27143400 & -1.59820000 \\
\hline $\mathrm{H}$ & 1.57631400 & 1.81599200 & 1.20126700 \\
\hline $\mathrm{H}$ & 2.80378400 & -1.59908200 & -1.07909600 \\
\hline $\mathrm{H}$ & 3.11426400 & 3.25278400 & -0.11172000 \\
\hline $\mathrm{H}$ & 4.37347700 & -0.17598200 & -2.36832500 \\
\hline C & 0.26529400 & -2.81485600 & 1.14245300 \\
\hline $\mathrm{H}$ & 0.32810700 & -3.79015400 & 0.63978200 \\
\hline $\mathrm{H}$ & 4.52482500 & 2.25712000 & -1.90125200 \\
\hline C & -0.85481000 & -1.95270500 & 0.61577700 \\
\hline $\mathrm{H}$ & 0.16095700 & -1.76262100 & -1.38188200 \\
\hline C & 0.20884200 & -2.97827100 & 2.64698100 \\
\hline $\mathrm{H}$ & 0.23400300 & -1.99657800 & 3.13314100 \\
\hline $\mathrm{H}$ & -0.70861900 & -3.49544300 & 2.94965100 \\
\hline $\mathrm{H}$ & 1.06816000 & -3.55553400 & 3.00055300 \\
\hline C & -0.91819900 & 0.68230400 & 0.84952900 \\
\hline C & -1.12038700 & 1.68661900 & -0.09585700 \\
\hline C & -1.24479200 & 0.91939600 & 2.18399000 \\
\hline C & -1.65421100 & 2.91132200 & 0.28700300 \\
\hline $\mathrm{H}$ & -0.83742600 & 1.50558700 & -1.13269900 \\
\hline C & -1.77280200 & 2.14482100 & 2.56905700 \\
\hline $\mathrm{H}$ & -1.07555600 & 0.13215300 & 2.91750000 \\
\hline C & -1.97878400 & 3.14221800 & 1.61961000 \\
\hline $\mathrm{H}$ & -1.80991400 & 3.68992900 & -0.45576200 \\
\hline $\mathrm{H}$ & -2.02699300 & 2.32299700 & 3.61115900 \\
\hline $\mathrm{H}$ & -2.39374200 & 4.10134200 & 1.92032200 \\
\hline $\mathrm{O}$ & 1.43796500 & -2.11809000 & 0.72643200 \\
\hline
\end{tabular}




$\begin{array}{llll}\mathrm{C} & -0.27211200 & -0.59966300 & 0.47015200 \\ \mathrm{H} & -1.87787200 & -2.07674900 & 0.96523000\end{array}$

\begin{tabular}{|c|c|c|c|}
\hline \multicolumn{4}{|c|}{ TS(cd)-VIII-(f) (-1015.047668 a.u.) } \\
\hline $\mathrm{C}$ & -2.27361300 & -1.44281600 & -0.46116200 \\
\hline $\mathrm{C}$ & -3.30053900 & -0.50716200 & -0.13856700 \\
\hline 0 & -4.48691600 & -0.86125400 & -0.71062400 \\
\hline $\mathrm{C}$ & -5.56797600 & -0.00551900 & -0.38792900 \\
\hline $\mathrm{H}$ & -5.36713600 & 1.02494300 & -0.70054200 \\
\hline $\mathrm{H}$ & -5.76171400 & 0.00213100 & 0.69058800 \\
\hline $\mathrm{H}$ & -6.43664400 & -0.39633500 & -0.92129700 \\
\hline 0 & -3.18368500 & 0.48403800 & 0.57226700 \\
\hline $\mathrm{N}$ & 1.23689100 & -0.78150800 & 0.72698200 \\
\hline $\mathrm{C}$ & 2.47090600 & -0.42203300 & 0.09920500 \\
\hline $\mathrm{C}$ & 4.94234700 & 0.21725000 & -1.01037900 \\
\hline $\mathrm{C}$ & 2.53251000 & 0.36929500 & -1.04637900 \\
\hline $\mathrm{C}$ & 3.64356600 & -0.89843500 & 0.68666600 \\
\hline $\mathrm{C}$ & 3.77188900 & 0.69396900 & -1.58796200 \\
\hline $\mathrm{C}$ & 4.87227200 & -0.58711600 & 0.12489300 \\
\hline $\mathrm{H}$ & 1.61891100 & 0.73499200 & -1.50772200 \\
\hline $\mathrm{H}$ & 3.56945400 & -1.51823500 & 1.57628600 \\
\hline $\mathrm{H}$ & 3.81638600 & 1.31574000 & -2.47892600 \\
\hline $\mathrm{H}$ & 5.78300200 & -0.96590400 & 0.58242400 \\
\hline $\mathrm{C}$ & 0.05632000 & -2.52877700 & -0.12008000 \\
\hline $\mathrm{H}$ & 0.44885000 & -2.57455600 & -1.15562300 \\
\hline $\mathrm{H}$ & 5.90724200 & 0.47047400 & -1.44230200 \\
\hline $\mathrm{C}$ & -0.86293900 & -1.32314600 & 0.00726900 \\
\hline $\mathrm{H}$ & -2.55897600 & -2.41936500 & -0.83931900 \\
\hline $\mathrm{C}$ & -0.48891600 & -3.86634100 & 0.30210600 \\
\hline $\mathrm{H}$ & -0.90896300 & -3.80718300 & 1.31288600 \\
\hline $\mathrm{H}$ & -1.26522000 & -4.21286800 & -0.38682300 \\
\hline $\mathrm{H}$ & 0.31407600 & -4.60908200 & 0.30707100 \\
\hline $\mathrm{C}$ & -0.18645200 & 1.17282400 & 0.34890200 \\
\hline $\mathrm{C}$ & -1.05333600 & 1.79695400 & -0.56132000 \\
\hline $\mathrm{C}$ & 0.56681200 & 1.96868200 & 1.22891700 \\
\hline C & -1.15496500 & 3.17991200 & -0.59289400 \\
\hline $\mathrm{H}$ & -1.61540300 & 1.19126700 & -1.26484200 \\
\hline $\mathrm{C}$ & 0.44465600 & 3.34710600 & 1.20418500 \\
\hline $\mathrm{H}$ & 1.23611500 & 1.48549700 & 1.93689900 \\
\hline $\mathrm{C}$ & -0.41526700 & 3.95712700 & 0.29202200 \\
\hline $\mathrm{H}$ & -1.82279900 & 3.65286100 & -1.30836000 \\
\hline $\mathrm{H}$ & 1.02113100 & 3.95263600 & 1.89916200 \\
\hline $\mathrm{H}$ & -0.50818700 & 5.04042300 & 0.27326700 \\
\hline 0 & 1.10369000 & -2.19326000 & 0.79133700 \\
\hline
\end{tabular}




$\begin{array}{lrrr}C & 0.00268600 & -0.25819300 & 0.31396200 \\ H & -1.59285600 & -1.51456100 & 0.90572200\end{array}$

\begin{tabular}{|c|c|c|c|}
\hline \multicolumn{4}{|c|}{ TS(cd)-VI-(c) (-1992.710611 a.u.) } \\
\hline $\mathrm{C}$ & -1.37037000 & 1.88708000 & -0.11479400 \\
\hline $\mathrm{C}$ & -1.36720400 & 3.36135200 & -0.01484300 \\
\hline 0 & -2.18225300 & 3.77489800 & 0.98013500 \\
\hline $\mathrm{C}$ & -2.22029700 & 5.18542100 & 1.16974600 \\
\hline $\mathrm{H}$ & -2.56711300 & 5.68632200 & 0.26077900 \\
\hline $\mathrm{H}$ & -1.22650200 & 5.57040200 & 1.41980200 \\
\hline $\mathrm{H}$ & -2.91564100 & 5.36343600 & 1.99103500 \\
\hline $\mathrm{O}$ & -0.73619300 & 4.11090600 & -0.72223500 \\
\hline 0 & -2.03998300 & -0.58884200 & 2.34293900 \\
\hline $\mathrm{N}$ & -2.59626700 & -1.12984500 & 1.13469000 \\
\hline $\mathrm{C}$ & -2.05266800 & -2.40370400 & 0.89050400 \\
\hline $\mathrm{C}$ & -0.99017500 & -4.95123700 & 0.45379300 \\
\hline $\mathrm{C}$ & -1.88273500 & -3.28529100 & 1.96219100 \\
\hline $\mathrm{C}$ & -1.69595800 & -2.80184100 & -0.40061200 \\
\hline $\mathrm{C}$ & -1.34493800 & -4.54670800 & 1.73882600 \\
\hline $\mathrm{C}$ & -1.17670000 & -4.07412300 & -0.61088300 \\
\hline $\mathrm{H}$ & -2.16450500 & -2.96359900 & 2.96032400 \\
\hline $\mathrm{H}$ & -1.79743400 & -2.10514600 & -1.22943000 \\
\hline $\mathrm{H}$ & -1.20786600 & -5.22320500 & 2.57959100 \\
\hline $\mathrm{H}$ & -0.89825200 & -4.37492600 & -1.61886600 \\
\hline $\mathrm{C}$ & -2.12186900 & 0.84464300 & 2.27745800 \\
\hline $\mathrm{H}$ & -0.57127200 & -5.93960800 & 0.28405600 \\
\hline $\mathrm{Rh}$ & 1.26309800 & 0.65131700 & -0.16380400 \\
\hline 0 & 2.09041000 & 2.01427200 & -1.45805200 \\
\hline $\mathrm{C}$ & 3.25801600 & 1.79165600 & -1.87737800 \\
\hline 0 & 4.00552000 & 0.80961900 & -1.61453500 \\
\hline $\mathrm{Rh}$ & 3.27207900 & -0.64381900 & -0.36635800 \\
\hline 0 & 2.41063200 & -1.60710500 & -1.96476200 \\
\hline $\mathrm{C}$ & 1.23632500 & -1.28834400 & -2.28326200 \\
\hline 0 & 0.49921200 & -0.40595100 & -1.75796200 \\
\hline $\mathrm{H}$ & 0.79275000 & -1.84348100 & -3.12558400 \\
\hline 0 & 4.00792000 & 0.38434300 & 1.24791600 \\
\hline $\mathrm{C}$ & 3.28195400 & 1.27575200 & 1.76342000 \\
\hline 0 & 2.11883200 & 1.62904900 & 1.42094200 \\
\hline $\mathrm{H}$ & 3.71351900 & 1.81507300 & 2.62112400 \\
\hline 0 & 2.43981000 & -2.04573800 & 0.87264900 \\
\hline $\mathrm{C}$ & 1.27706300 & -1.81695000 & 1.30543000 \\
\hline 0 & 0.56546700 & -0.79520500 & 1.09739100 \\
\hline $\mathrm{H}$ & 0.81906500 & -2.59562000 & 1.93717900 \\
\hline $\mathrm{H}$ & 3.67628200 & 2.54953600 & -2.55810700 \\
\hline
\end{tabular}




\begin{tabular}{|c|c|c|c|}
\hline C & -2.04034600 & 1.04241300 & 0.79844300 \\
\hline $\mathrm{H}$ & -0.52134800 & 1.55480800 & -0.74134300 \\
\hline C & -3.74651200 & -0.09699800 & -0.77487900 \\
\hline C & -5.01974000 & -0.15348200 & -0.19550700 \\
\hline C & -3.63210400 & -0.06473900 & -2.16368600 \\
\hline C & -6.15313900 & -0.14201400 & -0.99513400 \\
\hline $\mathrm{H}$ & -5.10429800 & -0.20813300 & 0.88800800 \\
\hline C & -4.77080600 & -0.04688600 & -2.96484800 \\
\hline $\mathrm{H}$ & -2.64211800 & -0.06249500 & -2.62082700 \\
\hline C & -6.03128600 & -0.08419100 & -2.38237100 \\
\hline $\mathrm{H}$ & -7.13797600 & -0.17470700 & -0.53484600 \\
\hline $\mathrm{H}$ & -4.66759400 & -0.01642000 & -4.04679600 \\
\hline $\mathrm{H}$ & -6.92123500 & -0.07723100 & -3.00739300 \\
\hline C & -2.55918300 & -0.09297400 & 0.15235900 \\
\hline $\mathrm{H}$ & -1.79103000 & 0.67271300 & -0.89092800 \\
\hline C & -1.01962800 & 1.42707900 & 3.12384300 \\
\hline $\mathrm{H}$ & -1.07778200 & 2.51979300 & 3.09309400 \\
\hline $\mathrm{H}$ & -1.12209200 & 1.10095000 & 4.16334800 \\
\hline $\mathrm{H}$ & -0.04182000 & 1.11100200 & 2.74622000 \\
\hline $\mathrm{H}$ & -3.11605000 & 1.17457400 & 2.64170500 \\
\hline
\end{tabular}

TS(cd)-VII-(c) (-1992.791544 a.u.)

$\begin{array}{lccc}\mathrm{C} & 0.40646900 & -0.37004000 & 1.58210900 \\ \mathrm{C} & 0.64992500 & 0.75534700 & 2.49580800 \\ \mathrm{O} & -0.29769800 & 0.87345000 & 3.42895500 \\ \mathrm{C} & -0.13194600 & 1.96152800 & 4.32904100 \\ \mathrm{H} & -0.16168000 & 2.91313400 & 3.78851000 \\ \mathrm{H} & 0.82206200 & 1.88965500 & 4.86076700 \\ \mathrm{H} & -0.96500600 & 1.90001400 & 5.02983500 \\ \mathrm{O} & 1.63235000 & 1.48184600 & 2.44473000 \\ \mathrm{~N} & 3.14872000 & -0.78039100 & -0.90749100 \\ \mathrm{C} & 4.53106700 & -0.81947300 & -0.54764700 \\ \mathrm{C} & 7.24111200 & -0.97121200 & 0.08237000 \\ \mathrm{C} & 5.10619100 & 0.14207300 & 0.28589100 \\ \mathrm{C} & 5.31065900 & -1.85509800 & -1.06824200 \\ \mathrm{C} & 6.46286100 & 0.06178800 & 0.58854900 \\ \mathrm{C} & 6.65654400 & -1.92975600 & -0.74337300 \\ \mathrm{H} & 4.51288000 & 0.95844200 & 0.69476800 \\ \mathrm{H} & 4.84220000 & -2.59777400 & -1.70699700 \\ \mathrm{H} & 6.90481400 & 0.81375000 & 1.23760800 \\ \mathrm{H} & 7.25665000 & -2.74345500 & -1.14358100 \\ \mathrm{C} & 1.84381800 & -2.10847600 & 0.39723100 \\ \mathrm{H} & 0.90565400 & -2.64477200 & 0.19996800 \\ \mathrm{H} & 8.29790500 & -1.03188400 & 0.32906500 \\ & & & \\ & & 5102 & \\ & & & \\ & & & \\ & & & \end{array}$




\begin{tabular}{|c|c|c|c|}
\hline $\mathrm{Rh}$ & -1.43975700 & -0.27023100 & 0.32222700 \\
\hline $\mathrm{O}$ & -1.32751400 & -2.31498300 & 0.03794200 \\
\hline C & -2.25614100 & -2.86807400 & -0.62510200 \\
\hline $\mathrm{O}$ & -3.25920900 & -2.33723800 & -1.15582400 \\
\hline $\mathrm{Rh}$ & -3.51590100 & -0.29374200 & -0.98139300 \\
\hline $\mathrm{O}$ & -2.35564100 & -0.00810400 & -2.66764500 \\
\hline C & -1.10992800 & 0.05562100 & -2.52945900 \\
\hline $\mathrm{O}$ & -0.43027200 & -0.01578900 & -1.46394200 \\
\hline $\mathrm{H}$ & -0.52077100 & 0.19165900 & -3.45179800 \\
\hline $\mathrm{O}$ & -4.51546300 & -0.57800900 & 0.79899900 \\
\hline C & -3.83816600 & -0.67300900 & 1.85393900 \\
\hline $\mathrm{O}$ & -2.58559500 & -0.59799600 & 2.00070200 \\
\hline $\mathrm{H}$ & -4.40931700 & -0.84518300 & 2.78191400 \\
\hline $\mathrm{O}$ & -3.61787000 & 1.74721300 & -0.70741200 \\
\hline C & -2.72304500 & 2.29714900 & -0.02175000 \\
\hline $\mathrm{O}$ & -1.72019300 & 1.76889500 & 0.54097200 \\
\hline $\mathrm{H}$ & -2.81599000 & 3.38854200 & 0.11420200 \\
\hline $\mathrm{H}$ & -2.15972900 & -3.96029400 & -0.74535200 \\
\hline C & 1.48105000 & -0.66256200 & 0.66483100 \\
\hline $\mathrm{H}$ & 0.06546000 & -1.25858400 & 2.12364200 \\
\hline C & 2.64216600 & -2.76391300 & 1.50790900 \\
\hline $\mathrm{H}$ & 3.57420400 & -2.22012300 & 1.70250200 \\
\hline $\mathrm{H}$ & 2.05639700 & -2.79007900 & 2.43363800 \\
\hline $\mathrm{H}$ & 2.89557700 & -3.79121500 & 1.23095400 \\
\hline C & 2.22096800 & 1.50117400 & -0.70306200 \\
\hline C & 3.13251900 & 1.92921100 & -1.68095600 \\
\hline C & 1.21909300 & 2.38066200 & -0.27984000 \\
\hline C & 3.05587900 & 3.21257800 & -2.19916300 \\
\hline $\mathrm{H}$ & 3.88798900 & 1.24449400 & -2.05538900 \\
\hline C & 1.13980300 & 3.65855200 & -0.81304500 \\
\hline $\mathrm{H}$ & 0.46956700 & 2.06801700 & 0.43132400 \\
\hline C & 2.06096400 & 4.08432100 & -1.76386800 \\
\hline $\mathrm{H}$ & 3.76919200 & 3.52811500 & -2.95624300 \\
\hline $\mathrm{H}$ & 0.34221100 & 4.31963100 & -0.48171200 \\
\hline $\mathrm{H}$ & 1.99766900 & 5.08875000 & -2.17597400 \\
\hline $\mathrm{O}$ & 2.55176600 & -2.06246500 & -0.84703800 \\
\hline C & 2.27625900 & 0.10666300 & -0.24733000 \\
\hline H & 2.52959500 & 0.01574800 & 1.10068100 \\
\hline
\end{tabular}

(cd)-E-(c) (-1992.839039 a.u.)

$\begin{array}{lccc}C & -0.32227400 & 2.06490300 & 0.71445700 \\ C & -0.83142200 & 3.13287100 & -0.14823300 \\ O & -2.03615300 & 3.56815300 & 0.26401100 \\ C & -2.72802900 & 4.39973100 & -0.65148900 \\ & & \end{array}$




\begin{tabular}{|c|c|c|c|}
\hline $\mathrm{H}$ & -2.91971800 & 3.85997300 & -1.58585600 \\
\hline $\mathrm{H}$ & -2.15619300 & 5.30540900 & -0.87867600 \\
\hline $\mathrm{H}$ & -3.67334200 & 4.65839700 & -0.17164000 \\
\hline $\mathrm{O}$ & -0.26989100 & 3.61552100 & -1.12235000 \\
\hline $\mathrm{N}$ & 3.00690400 & 0.64092500 & 0.88545000 \\
\hline C & 4.12578600 & -0.22529600 & 0.70779400 \\
\hline C & 6.24228100 & -1.95368000 & 0.38120300 \\
\hline C & 5.11664800 & 0.09493800 & -0.21168100 \\
\hline C & 4.18079200 & -1.37625500 & 1.48679000 \\
\hline C & 6.17777300 & -0.78668500 & -0.37576400 \\
\hline C & 5.24995600 & -2.24569700 & 1.31410600 \\
\hline $\mathrm{H}$ & 5.04223600 & 1.00898300 & -0.79531900 \\
\hline $\mathrm{H}$ & 3.38915600 & -1.57089100 & 2.20661400 \\
\hline $\mathrm{H}$ & 6.95940100 & -0.55789500 & -1.09497300 \\
\hline $\mathrm{H}$ & 5.30737900 & -3.15384600 & 1.90788900 \\
\hline C & 1.58240700 & 1.70602500 & 2.27074000 \\
\hline $\mathrm{H}$ & 0.89651800 & 0.93347300 & 2.65164400 \\
\hline $\mathrm{H}$ & 7.07534100 & -2.63877600 & 0.24665100 \\
\hline $\mathrm{Rh}$ & -1.29847600 & 0.16258100 & 0.28986400 \\
\hline 0 & -1.81536900 & 0.02754100 & 2.28984600 \\
\hline C & -2.47287100 & -0.98988700 & 2.65902600 \\
\hline $\mathrm{O}$ & -2.85240800 & -1.97442100 & 1.98112700 \\
\hline $\mathrm{Rh}$ & -2.39845300 & -2.03835800 & -0.04232900 \\
\hline $\mathrm{O}$ & -0.56326800 & -2.91342400 & 0.39766100 \\
\hline C & 0.39690100 & -2.17281400 & 0.69051500 \\
\hline $\mathrm{O}$ & 0.44148600 & -0.90540900 & 0.75394100 \\
\hline $\mathrm{H}$ & 1.35086000 & -2.68361700 & 0.93023100 \\
\hline $\mathrm{O}$ & -4.12839700 & -1.00130800 & -0.45553300 \\
\hline C & -4.08799000 & 0.25332100 & -0.42479800 \\
\hline $\mathrm{O}$ & -3.11440400 & 1.01579200 & -0.16395700 \\
\hline $\mathrm{H}$ & -5.03364200 & 0.77358900 & -0.65726600 \\
\hline $\mathrm{O}$ & -1.84512200 & -1.88946000 & -2.03452000 \\
\hline C & -1.25627500 & -0.84685200 & -2.41084500 \\
\hline $\mathrm{O}$ & -0.89931800 & 0.15869400 & -1.73236400 \\
\hline $\mathrm{H}$ & -1.00317100 & -0.79038600 & -3.48574500 \\
\hline $\mathrm{H}$ & -2.74276700 & -1.00513400 & 3.72988900 \\
\hline C & 1.19650200 & 2.02916800 & 0.82805300 \\
\hline $\mathrm{H}$ & -0.75118700 & 2.18174700 & 1.71740700 \\
\hline C & 1.67185100 & 2.88717900 & 3.19540300 \\
\hline $\mathrm{H}$ & 2.38765700 & 3.62202300 & 2.81031000 \\
\hline $\mathrm{H}$ & 0.68813500 & 3.36184500 & 3.27276100 \\
\hline $\mathrm{H}$ & 1.98296100 & 2.58245600 & 4.19874100 \\
\hline C & 2.03312300 & 0.65515300 & -1.32841300 \\
\hline C & 2.39142300 & -0.63894800 & -1.74289300 \\
\hline
\end{tabular}




$\begin{array}{lrrr}\mathrm{C} & 1.63932800 & 1.60496200 & -2.27505100 \\ \mathrm{C} & 2.37214000 & -0.96474800 & -3.08676500 \\ \mathrm{H} & 2.65388900 & -1.39687700 & -1.00963400 \\ \mathrm{C} & 1.65112600 & 1.27485200 & -3.62560100 \\ \mathrm{H} & 1.28159400 & 2.58004500 & -1.94986100 \\ \mathrm{C} & 2.01446800 & -0.00205900 & -4.03304900 \\ \mathrm{H} & 2.62773000 & -1.97352200 & -3.40000800 \\ \mathrm{H} & 1.34648900 & 2.01849400 & -4.35701900 \\ \mathrm{H} & 2.00585600 & -0.26040800 & -5.08964400 \\ \mathrm{O} & 2.90169200 & 1.10152300 & 2.18208700 \\ \mathrm{C} & 2.06230800 & 1.03774500 & 0.07575300 \\ \mathrm{H} & 1.61179600 & 3.01527100 & 0.53111100\end{array}$

TS(cd)-VIII-(c) (-1992.77121 a.u.)

$\begin{array}{lccc}\text { C } & -0.14720700 & 1.60966700 & -1.66525800 \\ \mathrm{C} & -0.03012000 & 2.92446800 & -1.00509500 \\ \mathrm{O} & 1.18107600 & 3.45282000 & -1.22709100 \\ \mathrm{C} & 1.42998900 & 4.67715100 & -0.54736500 \\ \mathrm{H} & 1.37764200 & 4.52394500 & 0.53538200 \\ \mathrm{H} & 0.69926000 & 5.44011800 & -0.83307700 \\ \mathrm{H} & 2.43558100 & 4.98164800 & -0.83880200 \\ \mathrm{O} & -0.91259200 & 3.47948800 & -0.38688300 \\ \mathrm{~N} & -3.31003500 & -0.41872000 & -1.24648700 \\ \mathrm{C} & -3.42668700 & -1.59445100 & -0.42377100 \\ \mathrm{C} & -3.65603400 & -3.92238300 & 1.12659700 \\ \mathrm{C} & -3.69598900 & -1.50635700 & 0.94717300 \\ \mathrm{C} & -3.30564200 & -2.85492900 & -1.01493500 \\ \mathrm{C} & -3.80277400 & -2.66615500 & 1.70639300 \\ \mathrm{C} & -3.41335600 & -4.00356500 & -0.23964600 \\ \mathrm{H} & -3.81857400 & -0.54509300 & 1.43303500 \\ \mathrm{H} & -3.12091400 & -2.92716800 & -2.08051300 \\ \mathrm{H} & -4.00655500 & -2.57579400 & 2.77107800 \\ \mathrm{H} & -3.30360700 & -4.97378900 & -0.71890700 \\ \mathrm{C} & -1.48263700 & -0.43191200 & -2.58490000 \\ \mathrm{H} & -0.89533900 & -1.26284300 & -2.15538700 \\ \mathrm{H} & -3.73881200 & -4.82291200 & 1.72935600 \\ \mathrm{Rh} & 1.29971500 & 0.21965000 & -0.33855700 \\ \mathrm{O} & 1.46925200 & -1.15341700 & -1.87424300 \\ \mathrm{C} & 2.19680800 & -2.17034700 & -1.67733100 \\ \mathrm{O} & 2.82727700 & -2.48958600 & -0.63981600 \\ \mathrm{Rh} & 2.71018600 & -1.24617300 & 0.99803400 \\ \mathrm{O} & 1.00764700 & -2.22984300 & 1.61217400 \\ \mathrm{C} & -0.09907700 & -1.80265000 & 1.19735400 \\ \mathrm{O} & -0.31196100 & -0.81701400 & 0.43054100 \\ & & & \\ & & & \\ & & & \\ & & & \\ & & & \end{array}$




$\begin{array}{lrrr}\text { H } & -1.00290300 & -2.34011600 & 1.53435200 \\ \mathrm{O} & 4.32799800 & -0.18737000 & 0.29464100 \\ \mathrm{C} & 4.12089600 & 0.71719800 & -0.55596600 \\ \mathrm{O} & 3.02118100 & 1.11435500 & -1.03148000 \\ \mathrm{H} & 5.01653800 & 1.23460600 & -0.93795700 \\ \mathrm{O} & 2.50412200 & 0.09964000 & 2.54325800 \\ \mathrm{C} & 1.85094500 & 1.15082200 & 2.33101200 \\ \mathrm{O} & 1.25413900 & 1.50671700 & 1.27341300 \\ \mathrm{H} & 1.78345400 & 1.86008800 & 3.17268300 \\ \mathrm{H} & 2.28321700 & -2.86315100 & -2.53065700 \\ \mathrm{C} & -1.40864000 & 0.75400300 & -1.64127300 \\ \mathrm{H} & 0.52357600 & 1.42234600 & -2.50499100 \\ \mathrm{C} & -1.10061200 & -0.20212500 & -4.02290800 \\ \mathrm{H} & -1.62003400 & 0.67294700 & -4.43243300 \\ \mathrm{H} & -0.01856900 & -0.07039000 & -4.12130400 \\ \mathrm{H} & -1.38374300 & -1.07506000 & -4.61863700 \\ \mathrm{C} & -2.97988500 & 1.42650300 & 0.33230500 \\ \mathrm{C} & -2.13829900 & 1.75766400 & 1.40051600 \\ \mathrm{C} & -4.34242500 & 1.75014400 & 0.39719400 \\ \mathrm{C} & -2.66115700 & 2.39426300 & 2.51742400 \\ \mathrm{H} & -1.08718600 & 1.48813600 & 1.36329700 \\ \mathrm{C} & -4.85303300 & 2.40412300 & 1.50874800 \\ \mathrm{H} & -4.99043900 & 1.47144100 & -0.43104000 \\ \mathrm{C} & -4.01333000 & 2.72081800 & 2.57437800 \\ \mathrm{H} & -2.00568700 & 2.63912900 & 3.34984400 \\ \mathrm{H} & -5.90892900 & 2.66012600 & 1.54894900 \\ \mathrm{H} & -4.41503200 & 3.22216800 & 3.45204400 \\ \mathrm{O} & -2.87950000 & -0.73420400 & -2.54786800 \\ \mathrm{C} & -2.50006200 & 0.66452200 & -0.81226200 \\ \mathrm{H} & -1.23736600 & 1.85265900 & -2.30082000\end{array}$

TS(cd)-VI-(o) (-1992.720277 a.u.)

$\begin{array}{lccc}\text { C } & 1.70281900 & -1.02934600 & -2.27269400 \\ \mathrm{C} & 0.92166800 & -1.94518800 & -1.45893700 \\ \mathrm{O} & 1.61583000 & -2.57212800 & -0.49868400 \\ \mathrm{C} & 0.86577800 & -3.41102500 & 0.38648600 \\ \mathrm{H} & 0.20668700 & -4.07531700 & -0.17756300 \\ \mathrm{H} & 0.26408400 & -2.79839700 & 1.06473100 \\ \mathrm{H} & 1.60886600 & -3.98534300 & 0.94361300 \\ \mathrm{O} & -0.27085400 & -2.20476400 & -1.67206800 \\ \mathrm{O} & 4.63810700 & -0.96800000 & -0.44736300 \\ \mathrm{~N} & 4.02132100 & 0.24759200 & 0.01247700 \\ \mathrm{C} & 3.75412600 & 0.12429500 & 1.41661000 \\ \mathrm{C} & 3.07455200 & 0.03608000 & 4.10629200 \\ & & & \end{array}$




\begin{tabular}{|c|c|c|c|}
\hline C & 4.03467900 & 1.21428200 & 2.23299600 \\
\hline C & 3.15769800 & -1.02351500 & 1.93908600 \\
\hline C & 3.67964900 & 1.17315000 & 3.57882200 \\
\hline C & 2.82380000 & -1.06461600 & 3.28617800 \\
\hline $\mathrm{H}$ & 4.50595100 & 2.08993100 & 1.79264300 \\
\hline $\mathrm{H}$ & 2.96108300 & -1.85825300 & 1.27301000 \\
\hline $\mathrm{H}$ & 3.88522000 & 2.02837900 & 4.21789100 \\
\hline $\mathrm{H}$ & 2.35431000 & -1.95674100 & 3.69663700 \\
\hline C & 4.40668100 & -1.03221300 & -1.86681900 \\
\hline $\mathrm{H}$ & 2.80439000 & 0.00280300 & 5.15914600 \\
\hline $\mathrm{Rh}$ & -1.61252700 & -0.79199600 & -0.50014800 \\
\hline $\mathrm{O}$ & -0.97205000 & 0.79498100 & -1.63193600 \\
\hline C & -1.41381800 & 1.94180400 & -1.34225900 \\
\hline $\mathrm{O}$ & -2.22756000 & 2.25709200 & -0.43325600 \\
\hline $\mathrm{Rh}$ & -2.99429100 & 0.74955200 & 0.73395400 \\
\hline $\mathrm{O}$ & -1.43527900 & 0.97991200 & 2.07021700 \\
\hline C & -0.37776100 & 0.33701200 & 1.85672700 \\
\hline $\mathrm{O}$ & -0.11943700 & -0.44211000 & 0.89349900 \\
\hline $\mathrm{H}$ & 0.43477800 & 0.45830700 & 2.59739200 \\
\hline $\mathrm{O}$ & -4.46047700 & 0.42567000 & -0.66189800 \\
\hline C & -4.22388400 & -0.39536900 & -1.58911000 \\
\hline $\mathrm{O}$ & -3.17324300 & -1.06150700 & -1.79621100 \\
\hline $\mathrm{H}$ & -5.03920600 & -0.55316600 & -2.31342800 \\
\hline $\mathrm{O}$ & -3.68152000 & -0.85492900 & 1.82310400 \\
\hline C & -3.20486300 & -1.99186700 & 1.56648300 \\
\hline $\mathrm{O}$ & -2.33697500 & -2.30137600 & 0.70353500 \\
\hline $\mathrm{H}$ & -3.59195300 & -2.82582600 & 2.17496100 \\
\hline $\mathrm{H}$ & -1.02955000 & 2.76922000 & -1.96152000 \\
\hline C & 2.99037300 & -0.54054300 & -1.87218500 \\
\hline $\mathrm{H}$ & 1.38134300 & -1.06379300 & -3.31789800 \\
\hline C & 2.25079600 & 1.74380000 & -0.93722500 \\
\hline C & 2.28360100 & 2.42644700 & -2.15538700 \\
\hline C & 1.66673500 & 2.35978400 & 0.17289000 \\
\hline C & 1.74062100 & 3.70245300 & -2.25829100 \\
\hline $\mathrm{H}$ & 2.74225500 & 1.95366800 & -3.02087300 \\
\hline C & 1.13318100 & 3.63878500 & 0.07168800 \\
\hline H & 1.63475500 & 1.83168400 & 1.12243700 \\
\hline C & 1.16718800 & 4.31306400 & -1.14601000 \\
\hline $\mathrm{H}$ & 1.77528900 & 4.22555000 & -3.21110700 \\
\hline $\mathrm{H}$ & 0.67143400 & 4.09820800 & 0.94222300 \\
\hline $\mathrm{H}$ & 0.74462600 & 5.31164400 & -1.22799800 \\
\hline C & 2.85075400 & 0.37468300 & -0.82015300 \\
\hline H & 1.56740000 & -0.19265900 & -0.91450200 \\
\hline C & 4.63468600 & -2.44816400 & -2.32085700 \\
\hline
\end{tabular}




$\begin{array}{llll}\mathrm{H} & 3.95306200 & -3.11964100 & -1.78904400 \\ \mathrm{H} & 4.44428100 & -2.53075200 & -3.39618600 \\ \mathrm{H} & 5.66556500 & -2.75802000 & -2.12728500 \\ \mathrm{H} & 5.08620800 & -0.32015100 & -2.37336800\end{array}$

TS(cd)-VII-(o) (-1992.784247 a.u.)

\begin{tabular}{lrrr} 
C & -1.66511800 & -2.31182000 & -1.98606800 \\
$\mathrm{C}$ & -0.43276300 & -2.67153800 & -1.41742900 \\
$\mathrm{O}$ & 0.27631400 & -3.55976000 & -2.13020900 \\
$\mathrm{C}$ & 1.52776200 & -3.95663000 & -1.57515400 \\
$\mathrm{H}$ & 1.40252500 & -4.32462400 & -0.55174300 \\
$\mathrm{H}$ & 2.23312200 & -3.12030900 & -1.56587800 \\
$\mathrm{H}$ & 1.89677400 & -4.75391400 & -2.22230800 \\
$\mathrm{O}$ & -0.00639100 & -2.22947200 & -0.32036800 \\
$\mathrm{~N}$ & -3.75838000 & 0.28671200 & -0.34356300 \\
$\mathrm{C}$ & -3.30802500 & 1.61761400 & -0.08243200 \\
$\mathrm{C}$ & -2.49181100 & 4.23826800 & 0.43542500 \\
$\mathrm{C}$ & -2.23673800 & 1.85988300 & 0.78054100 \\
$\mathrm{C}$ & -3.97615500 & 2.68764700 & -0.68102800 \\
$\mathrm{C}$ & -1.84014600 & 3.16806600 & 1.03910000 \\
$\mathrm{C}$ & -3.55624500 & 3.98736600 & -0.42622200 \\
$\mathrm{H}$ & -1.69377700 & 1.03165700 & 1.22833900 \\
$\mathrm{H}$ & -4.80316700 & 2.48820400 & -1.35477700 \\
$\mathrm{H}$ & -0.99246300 & 3.34420600 & 1.69916000 \\
$\mathrm{H}$ & -4.07409000 & 4.81398800 & -0.90715400 \\
$\mathrm{C}$ & -3.32936100 & -0.47829000 & -2.45164600 \\
$\mathrm{H}$ & -3.87316800 & -1.19601800 & -3.08105500 \\
$\mathrm{H}$ & -2.17027800 & 5.25758400 & 0.63164200 \\
$\mathrm{C}$ & -2.47880300 & -1.26908700 & -1.46690700 \\
$\mathrm{H}$ & -1.86994800 & -2.64170200 & -3.00115000 \\
$\mathrm{C}$ & -2.55005400 & 0.50286300 & -3.30078400 \\
$\mathrm{H}$ & -2.05018200 & 1.24249800 & -2.66691300 \\
$\mathrm{H}$ & -1.78022400 & -0.02280900 & -3.87652300 \\
$\mathrm{H}$ & -3.21849600 & 1.02654700 & -3.99049200 \\
$\mathrm{C}$ & -2.75930500 & -1.43261500 & 1.15518700 \\
$\mathrm{C}$ & -3.22170700 & -0.76277600 & 2.30135500 \\
$\mathrm{H}$ & -2.23228300 & -2.72350800 & 1.29313200 \\
$\mathrm{C}$ & -3.09040100 & -1.34067400 & 3.55216400 \\
$\mathrm{H}$ & -3.70003400 & 0.20784600 & 2.20521700 \\
$\mathrm{H}$ & -2.11382600 & -3.30113600 & 2.54944500 \\
$\mathrm{H}$ & -1.94106000 & -3.28180400 & 0.41340900 \\
$\mathrm{H}$ & -2.51692200 & -2.60494200 & 3.68263700 \\
$\mathrm{H}$ & -0.80787500 & 4.43008600 \\
$\mathrm{H}$ & -4.30159400 & 2.63944600 \\
\hline
\end{tabular}




$\begin{array}{lrrr}H & -2.40604600 & -3.05377100 & 4.66696400 \\ \mathrm{O} & -4.32112000 & 0.15893800 & -1.63744500 \\ \mathrm{C} & -2.92884300 & -0.83022100 & -0.15945900 \\ \mathrm{H} & -1.69909200 & -0.50141900 & -0.84285400 \\ \mathrm{Rh} & 1.32830800 & -0.50409200 & -0.08341800 \\ \mathrm{Rh} & 2.69701900 & 1.44482700 & 0.32923800 \\ \mathrm{O} & 2.35488600 & -0.77186200 & -1.84851000 \\ \mathrm{O} & 0.00929800 & 0.73079000 & -1.09164300 \\ \mathrm{O} & 2.72975100 & -1.62475100 & 0.90863800 \\ \mathrm{O} & 0.37309500 & -0.12889700 & 1.70113100 \\ \mathrm{O} & 4.02010400 & 0.20423100 & 1.29315500 \\ \mathrm{O} & 1.65924400 & 1.70002800 & 2.09508600 \\ \mathrm{O} & 3.63575600 & 1.06164300 & -1.46107200 \\ \mathrm{O} & 1.27404800 & 2.56258300 & -0.66486500 \\ \mathrm{C} & 3.25989900 & 0.06470400 & -2.12938400 \\ \mathrm{C} & 0.27562800 & 1.96959700 & -1.13895900 \\ \mathrm{C} & 3.74160100 & -1.02181600 & 1.36373700 \\ \mathrm{C} & 0.75705700 & 0.86802500 & 2.37431600 \\ \mathrm{H} & 0.21952300 & 1.03130600 & 3.32520100 \\ \mathrm{H} & -0.46493300 & 2.60002100 & -1.66243600 \\ \mathrm{H} & 3.78463100 & -0.10522100 & -3.08374500 \\ \mathrm{H} & 4.47484800 & -1.65452800 & 1.89001700\end{array}$

(cd)-E-(o) (-1992.814647 a.u.)

$\begin{array}{lrrr}\text { C } & -0.98898200 & 2.62725400 & 1.61619100 \\ \mathrm{C} & 0.13794100 & 2.96343300 & 0.90322900 \\ \mathrm{O} & 0.65364400 & 4.19338900 & 1.16548600 \\ \mathrm{C} & 1.80287200 & 4.55875900 & 0.42410100 \\ \mathrm{H} & 1.57783500 & 4.66026000 & -0.64463700 \\ \mathrm{H} & 2.60697400 & 3.82243900 & 0.53316500 \\ \mathrm{H} & 2.12016400 & 5.52496500 & 0.82426700 \\ \mathrm{O} & 0.69785000 & 2.27814400 & -0.02206200 \\ \mathrm{~N} & -3.20287800 & -0.21403400 & 1.08552800 \\ \mathrm{C} & -3.96910500 & -1.25801000 & 0.49443100 \\ \mathrm{C} & -5.42411200 & -3.31610200 & -0.62222300 \\ \mathrm{C} & -3.58783000 & -1.75799700 & -0.74891300 \\ \mathrm{C} & -5.04993100 & -1.77832900 & 1.19712700 \\ \mathrm{C} & -4.33031100 & -2.78977300 & -1.30629300 \\ \mathrm{C} & -5.77924600 & -2.81459700 & 0.62672400 \\ \mathrm{H} & -2.72256800 & -1.33720000 & -1.25775400 \\ \mathrm{H} & -5.29895000 & -1.37624200 & 2.17498200 \\ \mathrm{H} & -4.04514700 & -3.19172300 & -2.27472000 \\ \mathrm{H} & -6.62744800 & -3.23249200 & 1.16221900 \\ \mathrm{C} & -2.09579800 & 0.68870100 & 2.83250600 \\ & & & \\ & & 5109 & \end{array}$




\begin{tabular}{|c|c|c|c|}
\hline $\mathrm{H}$ & -2.85192600 & 1.38134700 & 3.24537500 \\
\hline $\mathrm{H}$ & -5.99794700 & -4.12731100 & -1.06276500 \\
\hline C & -1.51887400 & 1.25805500 & 1.54427500 \\
\hline $\mathrm{H}$ & -1.39846600 & 3.33194800 & 2.33568300 \\
\hline C & -1.08580100 & 0.28590300 & 3.86509500 \\
\hline $\mathrm{H}$ & -0.40404000 & -0.46033300 & 3.44442600 \\
\hline $\mathrm{H}$ & -0.49375200 & 1.16854900 & 4.12962100 \\
\hline $\mathrm{H}$ & -1.56210900 & -0.11346700 & 4.76502900 \\
\hline C & -2.95546400 & 1.40197200 & -0.70721700 \\
\hline C & -4.29055500 & 1.41056500 & -1.14877800 \\
\hline C & -1.95069800 & 1.99086900 & -1.48783300 \\
\hline C & -4.61120000 & 1.98931400 & -2.36333700 \\
\hline $\mathrm{H}$ & -5.07390100 & 0.98514100 & -0.52451900 \\
\hline C & -2.28589000 & 2.54095500 & -2.72021400 \\
\hline $\mathrm{H}$ & -0.91340500 & 1.98554300 & -1.14861800 \\
\hline C & -3.60547900 & 2.54606300 & -3.15589400 \\
\hline $\mathrm{H}$ & -5.64581900 & 2.01112900 & -2.69571200 \\
\hline $\mathrm{H}$ & -1.50247400 & 2.97686500 & -3.33453300 \\
\hline $\mathrm{H}$ & -3.85981300 & 2.99270900 & -4.11446500 \\
\hline $\mathrm{O}$ & -2.80285700 & -0.50472800 & 2.38669300 \\
\hline C & -2.61017800 & 0.83247900 & 0.57559600 \\
\hline $\mathrm{H}$ & -0.70405300 & 0.57841200 & 1.23018400 \\
\hline $\mathrm{Rh}$ & 1.58940000 & 0.31455200 & -0.10538500 \\
\hline $\mathrm{Rh}$ & 2.83415800 & -1.76007400 & -0.35213700 \\
\hline $\mathrm{O}$ & 1.63822100 & 0.01108900 & 1.93362700 \\
\hline $\mathrm{O}$ & -0.15692600 & -0.84872100 & -0.26911200 \\
\hline $\mathrm{O}$ & 3.38577500 & 1.28895700 & 0.01395500 \\
\hline $\mathrm{O}$ & 1.60373400 & 0.49412700 & -2.15534100 \\
\hline $\mathrm{O}$ & 4.54922500 & -0.62927100 & -0.33768400 \\
\hline $\mathrm{O}$ & 2.68003200 & -1.49387700 & -2.38973600 \\
\hline $\mathrm{O}$ & 2.89744600 & -1.86635200 & 1.70968000 \\
\hline $\mathrm{O}$ & 1.03920900 & -2.77751700 & -0.33858900 \\
\hline C & 2.29060800 & -0.98262300 & 2.36466600 \\
\hline C & -0.01871200 & -2.10698800 & -0.30681300 \\
\hline C & 4.43779700 & 0.60915400 & -0.14467900 \\
\hline C & 2.12823600 & -0.44518400 & -2.81528800 \\
\hline $\mathrm{H}$ & 2.09562200 & -0.32937300 & -3.91193500 \\
\hline $\mathrm{H}$ & -0.96041900 & -2.68703100 & -0.31472700 \\
\hline $\mathrm{H}$ & 2.32337600 & -1.08375000 & 3.46367600 \\
\hline $\mathrm{H}$ & 5.37976900 & 1.18117400 & -0.10853900 \\
\hline
\end{tabular}

TS(cd)-VIII-(o) (-1992.766341 a.u.)

$\begin{array}{lrrr}\text { C } & -0.79688500 & 2.61830600 & 1.69745800 \\ \text { C } & 0.17700600 & 2.99519500 & 0.74367200\end{array}$




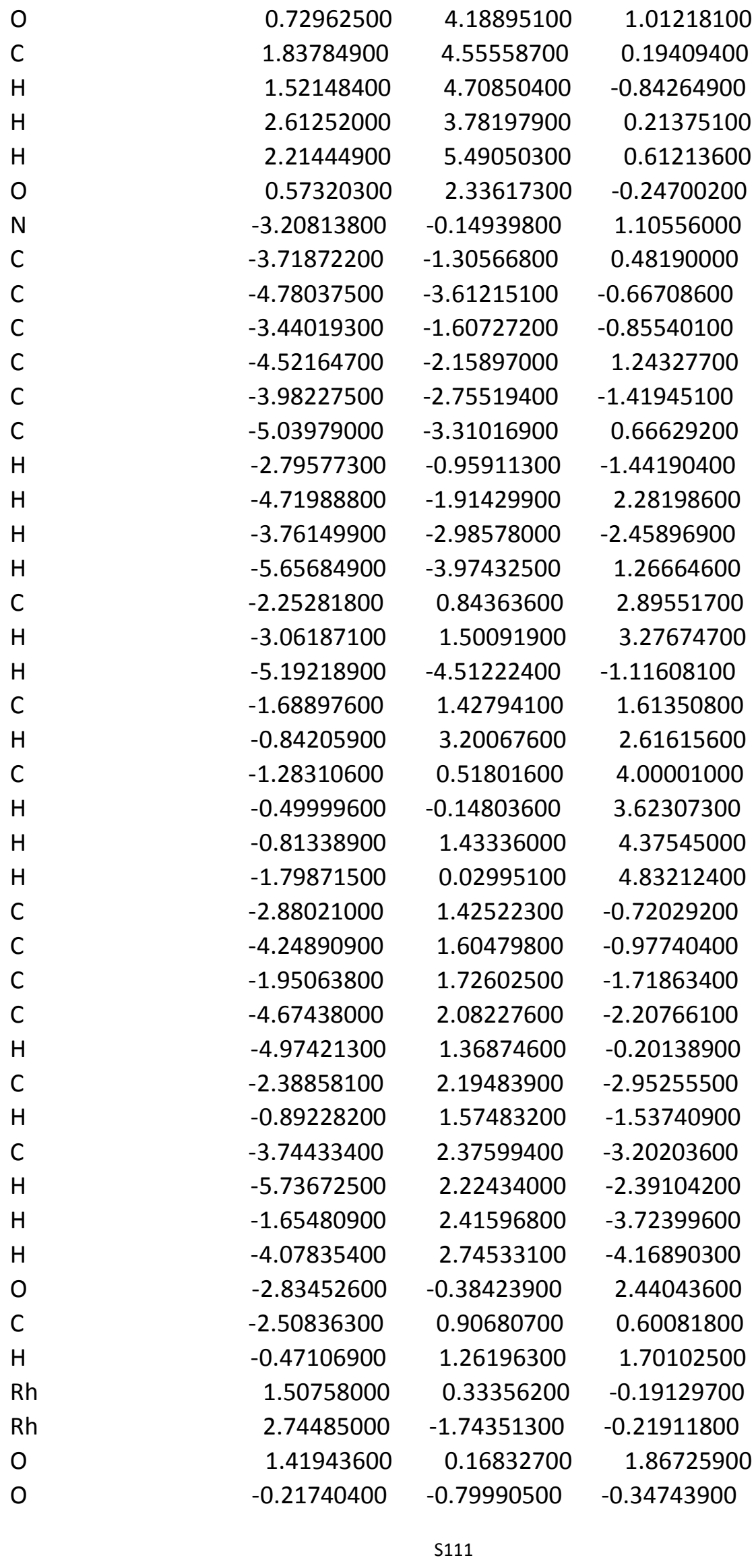




$\begin{array}{lccc}\mathrm{O} & 3.30672300 & 1.31972700 & -0.03308100 \\ \mathrm{O} & 1.64497000 & 0.37602900 & -2.23709300 \\ \mathrm{O} & 4.47001400 & -0.62389000 & -0.17166700 \\ \mathrm{O} & 2.72725000 & -1.62023400 & -2.27068600 \\ \mathrm{O} & 2.67582000 & -1.72021000 & 1.84427700 \\ \mathrm{O} & 0.95119800 & -2.74261500 & -0.25356300 \\ \mathrm{C} & 2.03052200 & -0.80292300 & 2.40520200 \\ \mathrm{C} & -0.10241800 & -2.05932700 & -0.31728200 \\ \mathrm{C} & 4.36141500 & 0.62579800 & -0.07621200 \\ \mathrm{C} & 2.20437100 & -0.60544900 & -2.80152900 \\ \mathrm{H} & 2.23731400 & -0.56443200 & -3.90257200 \\ \mathrm{H} & -1.05114700 & -2.62211900 & -0.35509600 \\ \mathrm{H} & 1.98498400 & -0.83972300 & 3.50693800 \\ \mathrm{H} & 5.30600600 & 1.19173800 & -0.02244800\end{array}$

TS(cx)-V (-1992.783361 a.u.)

$\begin{array}{lrrr}\text { C } & 0.53841100 & 0.60564700 & -0.03809600 \\ \text { C } & 0.80948600 & 1.67586800 & -1.04001900 \\ \text { O } & 0.76356000 & 2.88095000 & -0.46861900 \\ \text { C } & 0.85360400 & 3.98243300 & -1.37000400 \\ \text { H } & -0.01485100 & 3.98406700 & -2.03604600 \\ \text { H } & 1.77005600 & 3.92002300 & -1.96518600 \\ \text { H } & 0.86431100 & 4.87755500 & -0.74814500 \\ \text { O } & 1.00179500 & 1.45387700 & -2.21162200 \\ \text { N } & 3.80757300 & -0.92312000 & 0.70234000 \\ \text { C } & 4.08060900 & -2.11051100 & -0.02343100 \\ \text { C } & 4.73349200 & -4.41111700 & -1.47094200 \\ \text { C } & 4.65743800 & -2.00369100 & -1.29395100 \\ \text { C } & 3.86817800 & -3.37197500 & 0.53533900 \\ \text { C } & 4.97029900 & -3.15058400 & -2.01098600 \\ \text { C } & 4.19107100 & -4.51033300 & -0.19442800 \\ \text { H } & 4.88787700 & -1.02287800 & -1.70705500 \\ \text { H } & 3.46300200 & -3.44953200 & 1.53944500 \\ \text { H } & 5.41316900 & -3.05362900 & -2.99948100 \\ \text { H } & 4.01388700 & -5.48845200 & 0.24730400 \\ \text { C } & 1.69721300 & -1.09937900 & 1.56472600 \\ \text { H } & 1.37979700 & -2.04016100 & 1.08315800 \\ \text { H } & 4.98150900 & -5.30625900 & -2.03507300 \\ \text { Rh } & -1.48720900 & 0.10105200 & 0.02954600 \\ \text { O } & -1.83330700 & 0.79204300 & 1.94691900 \\ \text { C } & -3.01189700 & 0.69627200 & 2.40472000 \\ \text { O } & -4.02978800 & 0.21906700 & 1.85140800 \\ \text { Rh } & -3.86658200 & -0.50001600 & -0.08091700 \\ \text { O } & -3.33478300 & -2.36148700 & 0.63793900 \\ & & & \\ & & 5112 & \\ & & & \\ & & & \\ & & & \\ & & & \end{array}$




\begin{tabular}{|c|c|c|c|}
\hline C & -2.12606300 & -2.58930300 & 0.87181700 \\
\hline $\mathrm{O}$ & -1.13449700 & -1.80763100 & 0.75081700 \\
\hline $\mathrm{H}$ & -1.87975400 & -3.60120000 & 1.23566200 \\
\hline $\mathrm{O}$ & -4.21549500 & 1.39786000 & -0.80718000 \\
\hline C & -3.23489000 & 2.16013500 & -0.97742300 \\
\hline $\mathrm{O}$ & -2.01034600 & 1.94164600 & -0.73887000 \\
\hline $\mathrm{H}$ & -3.45735400 & 3.15944100 & -1.38798300 \\
\hline $\mathrm{O}$ & -3.52911200 & -1.16235800 & -2.00115600 \\
\hline C & -2.35846600 & -1.08698300 & -2.44934600 \\
\hline $\mathrm{O}$ & -1.32066800 & -0.64073000 & -1.88030500 \\
\hline $\mathrm{H}$ & -2.20152000 & -1.45702500 & -3.47590600 \\
\hline $\mathrm{H}$ & -3.14665800 & 1.08511900 & 3.42814200 \\
\hline C & 1.65477500 & -0.01195400 & 0.51606100 \\
\hline $\mathrm{H}$ & 1.07508700 & 1.02036000 & 1.09787300 \\
\hline C & 0.94238300 & -0.84525600 & 2.84408900 \\
\hline $\mathrm{H}$ & 1.29231900 & 0.08592600 & 3.30725000 \\
\hline $\mathrm{H}$ & -0.12997300 & -0.77032700 & 2.65594800 \\
\hline $\mathrm{H}$ & 1.12715500 & -1.66404500 & 3.54505000 \\
\hline C & 3.66900900 & 1.51979900 & 0.22329400 \\
\hline C & 3.82717900 & 2.01742600 & 1.51860500 \\
\hline C & 4.03111800 & 2.30558800 & -0.86775400 \\
\hline C & 4.32148900 & 3.29988000 & 1.71390600 \\
\hline $\mathrm{H}$ & 3.57491000 & 1.38428100 & 2.36898100 \\
\hline C & 4.52897400 & 3.59079100 & -0.66970600 \\
\hline $\mathrm{H}$ & 3.90527500 & 1.91467400 & -1.87741800 \\
\hline C & 4.66606000 & 4.09150000 & 0.61971400 \\
\hline $\mathrm{H}$ & 4.44126400 & 3.68481500 & 2.72376000 \\
\hline $\mathrm{H}$ & 4.80882300 & 4.19996500 & -1.52629500 \\
\hline $\mathrm{H}$ & 5.04989900 & 5.09687500 & 0.77571100 \\
\hline $\mathrm{O}$ & 3.09457500 & -1.15003100 & 1.89167400 \\
\hline C & 3.08651200 & 0.14882300 & 0.00250800 \\
\hline $\mathrm{H}$ & 3.05796800 & -0.06845000 & -1.07458800 \\
\hline
\end{tabular}

(cx)-D (-1015.140529 a.u.)

$\begin{array}{lrrr}\mathrm{C} & -0.32797700 & -1.74587000 & 1.17163200 \\ \mathrm{C} & -0.62897900 & -2.71439600 & 0.11093800 \\ \mathrm{O} & -0.58994400 & -3.96781900 & 0.59676600 \\ \mathrm{C} & -0.84939700 & -4.99059600 & -0.35837500 \\ \mathrm{H} & -0.10721400 & -4.96779700 & -1.16211200 \\ \mathrm{H} & -1.84203900 & -4.86476200 & -0.80158100 \\ \mathrm{H} & -0.79192400 & -5.93410100 & 0.18512400 \\ \mathrm{O} & -0.87189200 & -2.46463000 & -1.05287900 \\ \mathrm{~N} & 0.78389600 & 1.36986700 & -0.07583100 \\ \mathrm{C} & 2.13089000 & 0.99038700 & -0.32417000 \\ & & & \end{array}$




\begin{tabular}{lccc} 
C & 4.78830400 & 0.33103000 & -0.90416000 \\
$\mathrm{C}$ & 2.46853900 & 0.44775000 & -1.56864100 \\
$\mathrm{C}$ & 3.13523500 & 1.22799200 & 0.61522600 \\
$\mathrm{C}$ & 3.78686900 & 0.11583700 & -1.84634200 \\
$\mathrm{C}$ & 4.45226900 & 0.89528000 & 0.32030200 \\
$\mathrm{H}$ & 1.70269500 & 0.30405800 & -2.32718400 \\
$\mathrm{H}$ & 2.87497000 & 1.68568900 & 1.56422300 \\
$\mathrm{H}$ & 4.03226100 & -0.30984900 & -2.81662400 \\
$\mathrm{H}$ & 5.22382800 & 1.08292600 & 1.06381300 \\
$\mathrm{C}$ & 0.23149700 & 0.55128000 & 2.01224100 \\
$\mathrm{H}$ & 1.12994300 & 0.17837100 & 2.53366900 \\
$\mathrm{H}$ & 5.81962800 & 0.07128800 & -1.12784200 \\
$\mathrm{C}$ & -0.18369400 & -0.43451400 & 0.95826600 \\
$\mathrm{H}$ & -0.16510500 & -2.15700800 & 2.16715000 \\
$\mathrm{C}$ & -0.85989200 & 0.90618100 & 2.99779600 \\
$\mathrm{H}$ & -1.75690300 & 1.23410400 & 2.46153400 \\
$\mathrm{H}$ & -1.11308000 & 0.03907600 & 3.61683200 \\
$\mathrm{H}$ & -0.52695200 & 1.71711400 & 3.65290700 \\
$\mathrm{C}$ & -1.61327700 & 0.98797900 & -0.54417500 \\
$\mathrm{C}$ & -1.76436600 & 2.37136800 & -0.61616100 \\
$\mathrm{C}$ & -2.73679500 & 0.17002100 & -0.67550500 \\
$\mathrm{C}$ & -3.02260200 & 2.92834200 & -0.81822400 \\
$\mathrm{H}$ & -0.88835200 & 3.00487200 & -0.50947400 \\
$\mathrm{C}$ & -3.99209600 & 0.73002600 & -0.87801000 \\
$\mathrm{H}$ & -2.61723900 & -0.91031800 & -0.64103900 \\
$\mathrm{C}$ & -4.14007400 & 2.11166000 & -0.94711900 \\
$\mathrm{H}$ & -3.12794700 & 4.00960700 & -0.87165200 \\
$\mathrm{H}$ & -4.85896300 & 0.08177200 & -0.98444100 \\
$\mathrm{H}$ & -5.12343900 & 2.54963600 & -1.10216600 \\
$\mathrm{O}$ & 0.57082200 & 1.73923900 & 1.27236600 \\
$\mathrm{C}$ & -0.24884800 & 0.35569300 & -0.32550600 \\
$\mathrm{H}$ & 0.02304300 & -0.27078000 & -1.18041500 \\
& & & \\
\hline & & & \\
& & &
\end{tabular}

TS(cx)-VI-(f) (-1015.00229 a.u.)

$\begin{array}{lrrr}\mathrm{C} & 0.24208600 & 2.00614100 & 0.90151700 \\ \mathrm{C} & -0.85011800 & 2.52667200 & 0.03221700 \\ \mathrm{O} & -0.89875600 & 3.86934600 & 0.16551300 \\ \mathrm{C} & -1.90158000 & 4.50481500 & -0.61907300 \\ \mathrm{H} & -2.89936600 & 4.16204800 & -0.32883600 \\ \mathrm{H} & -1.75956300 & 4.28891400 & -1.68249900 \\ \mathrm{H} & -1.79867200 & 5.57429600 & -0.43081200 \\ \mathrm{O} & -1.60815900 & 1.90751200 & -0.67956700 \\ \mathrm{~N} & -0.02204100 & -1.46028600 & 0.39734500 \\ \mathrm{C} & -1.29100100 & -1.64535200 & -0.21512600 \\ & & & \end{array}$




$\begin{array}{lrrr}\text { C } & -3.70370900 & -2.12478200 & -1.52962800 \\ \mathrm{C} & -1.45546300 & -1.24624900 & -1.54343400 \\ \mathrm{C} & -2.32920500 & -2.29259400 & 0.45211100 \\ \mathrm{C} & -2.65732800 & -1.48701600 & -2.19066800 \\ \mathrm{C} & -3.53048000 & -2.52259600 & -0.20957400 \\ \mathrm{H} & -0.64433400 & -0.73428900 & -2.05793200 \\ \mathrm{H} & -2.18402500 & -2.60987500 & 1.47993600 \\ \mathrm{H} & -2.77943100 & -1.16277400 & -3.22140700 \\ \mathrm{H} & -4.33912200 & -3.02232400 & 0.31952600 \\ \mathrm{C} & -0.26237900 & -0.21696600 & 2.37187000 \\ \mathrm{H} & -1.33876700 & -0.00857200 & 2.51744600 \\ \mathrm{H} & -4.64569400 & -2.30738800 & -2.04034500 \\ \mathrm{C} & 0.26670900 & 0.63699200 & 1.27039400 \\ \mathrm{H} & 0.58148300 & 2.77665500 & 1.60117900 \\ \mathrm{C} & 0.49807800 & -0.14325000 & 3.67483600 \\ \mathrm{H} & 1.56264100 & -0.32558500 & 3.49222600 \\ \mathrm{H} & 0.37995100 & 0.84296000 & 4.13602000 \\ \mathrm{H} & 0.12871100 & -0.90046600 & 4.37280100 \\ \mathrm{C} & 2.01567300 & -0.27375800 & -0.38959000 \\ \mathrm{C} & 2.88254500 & -1.18430400 & 0.22472300 \\ \mathrm{C} & 2.45297000 & 0.44736200 & -1.49599000 \\ \mathrm{C} & 4.17727800 & -1.33768200 & -0.24713400 \\ \mathrm{H} & 2.52154900 & -1.76677800 & 1.07032300 \\ \mathrm{C} & 3.75500300 & 0.29476700 & -1.96556000 \\ \mathrm{H} & 1.76580500 & 1.12447200 & -2.00244100 \\ \mathrm{C} & 4.61860200 & -0.59456600 & -1.34078900 \\ \mathrm{H} & 4.84883800 & -2.03949000 & 0.24182700 \\ \mathrm{H} & 4.08625900 & 0.86437600 & -2.83032400 \\ \mathrm{H} & 5.63382600 & -0.71922900 & -1.70972600 \\ \mathrm{O} & -0.08925000 & -1.53862200 & 1.82409100 \\ \mathrm{C} & 0.62296500 & -0.16193300 & 0.17772900 \\ \mathrm{H} & 0.49963900 & 1.16496000 & -0.34165300\end{array}$

TS(cx)-VII-(f) (-1015.056775 a.u.)

$\begin{array}{lrrr}\text { C } & 2.23012800 & -1.47761700 & 0.57078000 \\ \text { C } & 3.20898600 & -0.56403400 & 0.09928100 \\ \text { O } & 4.47365600 & -0.97841600 & 0.42528300 \\ \text { C } & 5.50569800 & -0.16777300 & -0.09825600 \\ \text { H } & 5.42473600 & 0.86529700 & 0.25902900 \\ \text { H } & 5.48122400 & -0.14352000 & -1.19393900 \\ \text { H } & 6.44551500 & -0.60785700 & 0.24279500 \\ \text { O } & 3.02160600 & 0.45786600 & -0.55340200 \\ \text { N } & -1.14080400 & -0.74411100 & -0.63272100 \\ \text { C } & -2.44905500 & -0.41865200 & -0.16381300 \\ & & & \end{array}$




\begin{tabular}{lrcc} 
C & -5.03531200 & 0.15308700 & 0.67902300 \\
$\mathrm{C}$ & -2.64995400 & 0.44327000 & 0.91284500 \\
$\mathrm{C}$ & -3.53573800 & -0.99952800 & -0.81786000 \\
$\mathrm{C}$ & -3.94756700 & 0.73003600 & 1.32274300 \\
$\mathrm{C}$ & -4.82328900 & -0.71646600 & -0.38830000 \\
$\mathrm{H}$ & -1.80258600 & 0.89748200 & 1.42043800 \\
$\mathrm{H}$ & -3.35269200 & -1.67729700 & -1.64650800 \\
$\mathrm{H}$ & -4.10283500 & 1.40501500 & 2.16066100 \\
$\mathrm{H}$ & -5.66892300 & -1.17461800 & -0.89507200 \\
$\mathrm{C}$ & -0.10357400 & -2.52530100 & 0.27159200 \\
$\mathrm{H}$ & -0.71324300 & -2.66084800 & 1.18330700 \\
$\mathrm{H}$ & -6.04659000 & 0.37870700 & 1.00720600 \\
$\mathrm{C}$ & 0.83401500 & -1.30388800 & 0.41186800 \\
$\mathrm{H}$ & 2.57086500 & -2.39128500 & 1.04670700 \\
$\mathrm{C}$ & 0.55403800 & -3.81381900 & -0.13265100 \\
$\mathrm{H}$ & 1.19247300 & -3.65479400 & -1.00626900 \\
$\mathrm{H}$ & 1.16834900 & -4.20651900 & 0.68283900 \\
$\mathrm{H}$ & -0.21167700 & -4.55720400 & -0.37447100 \\
$\mathrm{C}$ & 0.23515400 & 1.22717600 & -0.16476700 \\
$\mathrm{C}$ & -0.45189800 & 2.00685200 & -1.10592700 \\
$\mathrm{C}$ & 1.07907300 & 1.85901000 & 0.75996400 \\
$\mathrm{C}$ & -0.29220200 & 3.38389800 & -1.12293300 \\
$\mathrm{H}$ & -1.09385900 & 1.51928200 & -1.83532000 \\
$\mathrm{C}$ & 1.21749300 & 3.23665600 & 0.74800900 \\
$\mathrm{H}$ & 1.61354200 & 1.26636000 & 1.49807900 \\
$\mathrm{C}$ & 0.53752400 & 4.00408200 & -0.19413100 \\
$\mathrm{H}$ & -0.81932800 & 3.97584400 & -1.86682400 \\
$\mathrm{H}$ & 1.86965300 & 3.71481500 & 1.47414200 \\
$\mathrm{H}$ & 0.65736000 & 5.08458800 & -0.20540600 \\
$\mathrm{O}$ & -0.96734200 & -2.13504500 & -0.80580600 \\
$\mathrm{C}$ & 0.02645900 & -0.20985100 & -0.14546300 \\
$\mathrm{H}$ & 0.31042100 & -0.66274700 & 1.29702500 \\
& & & \\
\hline & & & \\
& & &
\end{tabular}

(cx)-E-(f) (-1015.126495 a.u.)

$\begin{array}{lrrr}\mathrm{C} & 0.06507900 & -1.35409200 & 1.17568600 \\ \mathrm{C} & 1.46257200 & -0.91234100 & 1.46838200 \\ \mathrm{O} & 1.40302700 & 0.33307900 & 1.97251600 \\ \mathrm{C} & 2.66231200 & 0.93449000 & 2.25708800 \\ \mathrm{H} & 3.26039100 & 1.00355300 & 1.34235600 \\ \mathrm{H} & 3.21349600 & 0.35257400 & 3.00223600 \\ \mathrm{H} & 2.43956400 & 1.93334100 & 2.63387500 \\ \mathrm{O} & 2.48789000 & -1.53465400 & 1.33913200 \\ \mathrm{O} & 1.06874000 & -1.36154800 & -1.39598300 \\ \mathrm{~N} & 0.07847400 & -0.35553900 & -1.16322300 \\ & & & \end{array}$




\begin{tabular}{lrrr} 
C & 0.73943300 & 0.90019200 & -1.05010000 \\
$\mathrm{C}$ & 1.97642800 & 3.40527200 & -0.84949000 \\
$\mathrm{C}$ & 2.10512200 & 1.03355800 & -1.31227800 \\
$\mathrm{C}$ & -0.01057900 & 2.03454500 & -0.71937600 \\
$\mathrm{C}$ & 2.70958200 & 2.28347600 & -1.21388400 \\
$\mathrm{C}$ & 0.61201200 & 3.26861900 & -0.60729400 \\
$\mathrm{H}$ & 2.68836500 & 0.15936200 & -1.57676200 \\
$\mathrm{H}$ & -1.08035400 & 1.95030100 & -0.55271600 \\
$\mathrm{H}$ & 3.77439800 & 2.37060500 & -1.42062000 \\
$\mathrm{H}$ & 0.01578700 & 4.13734700 & -0.33710700 \\
$\mathrm{C}$ & 0.46009400 & -2.60484600 & -1.09111800 \\
$\mathrm{H}$ & 2.45640400 & 4.37718900 & -0.76783800 \\
$\mathrm{C}$ & -0.40678400 & -2.29058100 & 0.10174600 \\
$\mathrm{H}$ & -0.53473600 & -1.38864000 & 2.08560900 \\
$\mathrm{C}$ & -2.06905900 & -0.25867000 & 0.12055300 \\
$\mathrm{C}$ & -2.45611800 & 0.42851200 & 1.26925600 \\
$\mathrm{C}$ & -2.97349100 & -0.40240800 & -0.93327200 \\
$\mathrm{C}$ & -3.74108100 & 0.95129000 & 1.37310700 \\
$\mathrm{H}$ & -1.72839700 & 0.57993100 & 2.06595200 \\
$\mathrm{C}$ & -4.25452700 & 0.12219100 & -0.83046200 \\
$\mathrm{H}$ & -2.65045400 & -0.91939300 & -1.83578500 \\
$\mathrm{C}$ & -4.64193300 & 0.79669900 & 0.32535600 \\
$\mathrm{H}$ & -4.03502600 & 1.48995400 & 2.27081100 \\
$\mathrm{H}$ & -4.95525100 & 0.00602700 & -1.65387300 \\
$\mathrm{H}$ & -5.64552000 & 1.20769100 & 0.40482500 \\
$\mathrm{C}$ & -0.70201800 & -0.82501300 & -0.01116200 \\
$\mathrm{H}$ & -1.20913600 & -2.98136700 & 0.35244200 \\
$\mathrm{C}$ & 1.55108700 & -3.63124700 & -0.91557100 \\
$\mathrm{H}$ & 1.10818400 & -4.61092500 & -0.70770200 \\
$\mathrm{H}$ & 2.14551800 & -3.71267900 & -1.83094200 \\
$\mathrm{H}$ & 2.20300000 & -3.34447800 & -0.08577300 \\
$\mathrm{H}$ & -0.22159000 & -2.89451400 & -1.91473400 \\
& & & \\
\hline & & & \\
& & & \\
& & &
\end{tabular}

TS(cx)-VIII-(f) (-1015.045827 a.u.)

$\begin{array}{lrrr}\mathrm{C} & 2.22787100 & -1.45344400 & 0.56969200 \\ \mathrm{C} & 3.23955500 & -0.55224600 & 0.13087800 \\ \mathrm{O} & 4.46687300 & -0.93232800 & 0.59704700 \\ \mathrm{C} & 5.53996800 & -0.14274200 & 0.11935900 \\ \mathrm{H} & 5.42558500 & 0.90585600 & 0.41600100 \\ \mathrm{H} & 5.60520300 & -0.17688100 & -0.97398400 \\ \mathrm{H} & 6.44732200 & -0.56010700 & 0.56053600 \\ \mathrm{O} & 3.08592000 & 0.42753800 & -0.58556800 \\ \mathrm{~N} & -1.18255400 & -0.73239800 & -0.74425300 \\ \mathrm{C} & -2.44793800 & -0.42129200 & -0.15294300 \\ & & \\ & & \mathrm{~S} 117 & \end{array}$




\begin{tabular}{lrcc} 
C & -4.96556600 & 0.13108500 & 0.89755300 \\
$\mathrm{C}$ & -2.56408200 & 0.36088900 & 0.99466800 \\
$\mathrm{C}$ & -3.58906500 & -0.93211400 & -0.77296900 \\
$\mathrm{C}$ & -3.82629600 & 0.64088700 & 1.50784300 \\
$\mathrm{C}$ & -4.84054000 & -0.66212900 & -0.24102300 \\
$\mathrm{H}$ & -1.67475000 & 0.75582100 & 1.47950600 \\
$\mathrm{H}$ & -3.47289600 & -1.54604500 & -1.66189700 \\
$\mathrm{H}$ & -3.91325100 & 1.25525700 & 2.40078600 \\
$\mathrm{H}$ & -5.72674600 & -1.06691900 & -0.72371800 \\
$\mathrm{C}$ & -0.11876000 & -2.52180800 & 0.15158300 \\
$\mathrm{H}$ & -0.68427000 & -2.69223600 & 1.08713400 \\
$\mathrm{H}$ & -5.94881800 & 0.35004000 & 1.30603200 \\
$\mathrm{C}$ & 0.77860200 & -1.28354700 & 0.28489000 \\
$\mathrm{H}$ & 2.50179600 & -2.33980200 & 1.13139300 \\
$\mathrm{C}$ & 0.56231000 & -3.78236300 & -0.30737000 \\
$\mathrm{H}$ & 1.18092000 & -3.57755700 & -1.18671400 \\
$\mathrm{H}$ & 1.19853100 & -4.19611400 & 0.48099400 \\
$\mathrm{H}$ & -0.18951100 & -4.53312200 & -0.56842800 \\
$\mathrm{C}$ & 0.21894400 & 1.21104200 & -0.26791000 \\
$\mathrm{C}$ & -0.51318400 & 2.00199400 & -1.16984900 \\
$\mathrm{C}$ & 1.07460800 & 1.84901000 & 0.64424800 \\
$\mathrm{C}$ & -0.38578000 & 3.38078400 & -1.16167000 \\
$\mathrm{H}$ & -1.16835100 & 1.51393100 & -1.88704800 \\
$\mathrm{C}$ & 1.18294600 & 3.23058300 & 0.65882600 \\
$\mathrm{H}$ & 1.64330300 & 1.26211400 & 1.36071800 \\
$\mathrm{C}$ & 0.45922500 & 4.00184300 & -0.24510500 \\
$\mathrm{H}$ & -0.94858100 & 3.97647900 & -1.87609800 \\
$\mathrm{H}$ & 1.84491800 & 3.70789600 & 1.37689800 \\
$\mathrm{H}$ & 0.55577000 & 5.08478200 & -0.23791500 \\
$\mathrm{O}$ & -1.02185300 & -2.13174400 & -0.88567200 \\
$\mathrm{C}$ & 0.01959800 & -0.22041000 & -0.24970700 \\
$\mathrm{H}$ & 1.03969100 & -0.92045900 & 1.37314100 \\
& & & \\
\hline & & & \\
& & &
\end{tabular}

TS(cx)-VI-(c) (-1992.713441 a.u.)

$\begin{array}{lrrr}\mathrm{C} & -0.99174800 & -1.89736900 & -0.05601900 \\ \mathrm{C} & -0.59082700 & -3.26766400 & 0.32953300 \\ \mathrm{O} & 0.05929100 & -3.89597300 & -0.65713400 \\ \mathrm{C} & 0.58447600 & -5.17451300 & -0.31461900 \\ \mathrm{H} & -0.20799200 & -5.83445000 & 0.05102000 \\ \mathrm{H} & 1.34741400 & -5.07198100 & 0.46365500 \\ \mathrm{H} & 1.02572600 & -5.57482200 & -1.22771700 \\ \mathrm{O} & -0.80269100 & -3.73924000 & 1.42618700 \\ \mathrm{O} & -1.78523100 & 0.74960700 & 2.10884600 \\ \mathrm{~N} & -2.68205400 & 0.94345400 & 1.00921000 \\ & & & \end{array}$




\begin{tabular}{|c|c|c|c|}
\hline C & -2.53718500 & 2.23765600 & 0.48133000 \\
\hline C & -2.27248000 & 4.82872600 & -0.53961100 \\
\hline C & -2.14165100 & 3.28496700 & 1.31889000 \\
\hline C & -2.81119700 & 2.49235600 & -0.86711600 \\
\hline C & -2.00334000 & 4.56679700 & 0.80108300 \\
\hline C & -2.68365900 & 3.78339600 & -1.36360400 \\
\hline $\mathrm{H}$ & -1.93175300 & 3.07998100 & 2.36317300 \\
\hline $\mathrm{H}$ & -3.10526800 & 1.68055700 & -1.52763400 \\
\hline $\mathrm{H}$ & -1.68241200 & 5.37125300 & 1.45921800 \\
\hline $\mathrm{H}$ & -2.89584000 & 3.96787100 & -2.41451700 \\
\hline C & -1.60699400 & -0.67060700 & 2.28444200 \\
\hline $\mathrm{H}$ & -2.16588200 & 5.83452700 & -0.93762500 \\
\hline $\mathrm{Rh}$ & 3.25209300 & 0.96172600 & -0.28776700 \\
\hline $\mathrm{O}$ & 3.11106200 & 1.40913900 & 1.70229500 \\
\hline C & 2.18064100 & 0.86213900 & 2.35096200 \\
\hline $\mathrm{O}$ & 1.30715400 & 0.05731300 & 1.92597000 \\
\hline $\mathrm{Rh}$ & 1.31920800 & -0.44972200 & -0.06926500 \\
\hline $\mathrm{O}$ & 2.58151100 & -2.01384000 & 0.37970000 \\
\hline C & 3.82027000 & -1.77247600 & 0.39044700 \\
\hline $\mathrm{O}$ & 4.40487100 & -0.68020500 & 0.15958600 \\
\hline $\mathrm{H}$ & 4.47286100 & -2.62662200 & 0.63225000 \\
\hline $\mathrm{O}$ & 0.17013600 & 1.17123800 & -0.54722500 \\
\hline C & 0.74418000 & 2.28105300 & -0.74085800 \\
\hline $\mathrm{O}$ & 1.98147900 & 2.52010100 & -0.70743200 \\
\hline $\mathrm{H}$ & 0.07636900 & 3.12929100 & -0.96740200 \\
\hline $\mathrm{O}$ & 1.48369100 & -0.87516300 & -2.07680700 \\
\hline C & 2.42693200 & -0.32925800 & -2.71590600 \\
\hline $\mathrm{O}$ & 3.30562700 & 0.45739600 & -2.27844000 \\
\hline $\mathrm{H}$ & 2.48371800 & -0.57688100 & -3.78757700 \\
\hline $\mathrm{H}$ & 2.12219300 & 1.11361700 & 3.42142800 \\
\hline C & -1.72217900 & -1.09672900 & 0.85882400 \\
\hline $\mathrm{H}$ & -0.36637300 & -1.46873500 & -0.86043800 \\
\hline C & -3.88638800 & -0.60219000 & -0.49399100 \\
\hline C & -3.94814100 & -1.16615100 & -1.76972400 \\
\hline C & -5.08543200 & -0.36175800 & 0.19127900 \\
\hline C & -5.16827400 & -1.54282000 & -2.32217000 \\
\hline H & -3.03613500 & -1.30119500 & -2.34967100 \\
\hline C & -6.30234100 & -0.72335000 & -0.36969600 \\
\hline $\mathrm{H}$ & -5.04935600 & 0.11775500 & 1.16640500 \\
\hline C & -6.34953900 & -1.32483700 & -1.62437400 \\
\hline $\mathrm{H}$ & -5.19151200 & -1.98768300 & -3.31410300 \\
\hline H & -7.22125000 & -0.53524700 & 0.18084700 \\
\hline H & -7.30445600 & -1.60613600 & -2.06177000 \\
\hline C & -2.60006500 & -0.22125500 & 0.19059200 \\
\hline
\end{tabular}




$\begin{array}{llrr}H & -1.79723600 & -0.98186600 & -0.87290100 \\ \mathrm{C} & -2.66512400 & -1.27946500 & 3.18010300 \\ \mathrm{H} & -3.66521100 & -1.14956900 & 2.75053800 \\ \mathrm{H} & -2.47339600 & -2.35210900 & 3.28911000 \\ \mathrm{H} & -2.64406500 & -0.80709400 & 4.16752100 \\ \mathrm{H} & -0.59909200 & -0.78766100 & 2.69502000\end{array}$

TS(cx)-VII-(c) (-1992.795071 a.u.)

\begin{tabular}{|c|c|c|c|}
\hline C & 0.04730200 & 1.27150000 & 1.56832400 \\
\hline C & -0.04626100 & 2.68958600 & 1.19453700 \\
\hline $\mathrm{O}$ & -1.18883500 & 3.22920000 & 1.65860800 \\
\hline C & -1.43013900 & 4.56231300 & 1.23595200 \\
\hline $\mathrm{H}$ & -1.50727900 & 4.60745800 & 0.14404700 \\
\hline $\mathrm{H}$ & -0.62512300 & 5.23119200 & 1.55701600 \\
\hline $\mathrm{H}$ & -2.37541700 & 4.85784700 & 1.69327900 \\
\hline 0 & 0.77331500 & 3.32626300 & 0.56333300 \\
\hline$N$ & 3.48021600 & -0.09119900 & 1.13439400 \\
\hline C & 4.01258900 & -1.19525900 & 0.39343600 \\
\hline C & 5.11713100 & -3.36576300 & -0.95199500 \\
\hline C & 3.57845600 & -1.50460600 & -0.89485800 \\
\hline C & 4.99815000 & -1.96618300 & 1.01030300 \\
\hline C & 4.14196800 & -2.58711700 & -1.56330900 \\
\hline C & 5.53886600 & -3.05261700 & 0.33831000 \\
\hline $\mathrm{H}$ & 2.81436500 & -0.90370000 & -1.38155900 \\
\hline $\mathrm{H}$ & 5.31509500 & -1.71157100 & 2.01758700 \\
\hline $\mathrm{H}$ & 3.80348800 & -2.82370300 & -2.56956700 \\
\hline $\mathrm{H}$ & 6.30088100 & -3.65649500 & 0.82483800 \\
\hline C & 1.68608300 & -0.54465800 & 2.42638200 \\
\hline $\mathrm{H}$ & 1.35843300 & -1.51925300 & 2.01876700 \\
\hline $\mathrm{H}$ & 5.54782400 & -4.21430500 & -1.47724100 \\
\hline $\mathrm{Rh}$ & -1.37934400 & 0.05982900 & 0.33068000 \\
\hline $\mathrm{O}$ & -1.25806400 & -1.49618800 & 1.68786400 \\
\hline C & -1.99137700 & -2.50936900 & 1.47912600 \\
\hline O & -2.78089100 & -2.72342700 & 0.52940200 \\
\hline $\mathrm{Rh}$ & -2.98261700 & -1.29177600 & -0.95211700 \\
\hline 0 & -1.35966100 & -2.12248300 & -1.95202900 \\
\hline C & -0.21105700 & -1.74018200 & -1.65240900 \\
\hline 0 & 0.12036100 & -0.85545900 & -0.79842900 \\
\hline $\mathrm{H}$ & 0.63100600 & -2.21625000 & -2.18432500 \\
\hline $\mathrm{O}$ & -4.46586000 & -0.38166500 & 0.14133300 \\
\hline C & -4.13070700 & 0.42628600 & 1.04441100 \\
\hline 0 & -2.97278200 & 0.80663100 & 1.37811800 \\
\hline $\mathrm{H}$ & -4.95408900 & 0.86838800 & 1.62981500 \\
\hline $\mathrm{O}$ & -3.04786400 & 0.26282500 & -2.31162500 \\
\hline
\end{tabular}




$\begin{array}{lrrr}\mathrm{C} & -2.36566700 & 1.29007900 & -2.08066300 \\ \mathrm{O} & -1.58988500 & 1.52515800 & -1.10719900 \\ \mathrm{H} & -2.44252100 & 2.10303200 & -2.82267200 \\ \mathrm{H} & -1.91611300 & -3.30577500 & 2.23904500 \\ \mathrm{C} & 1.31025400 & 0.57667000 & 1.44354200 \\ \mathrm{H} & -0.46218100 & 1.05331700 & 2.50775100 \\ \mathrm{C} & 1.19519600 & -0.37628900 & 3.83733100 \\ \mathrm{H} & 1.41519900 & 0.62893200 & 4.21162900 \\ \mathrm{H} & 0.11785400 & -0.55953200 & 3.88450600 \\ \mathrm{H} & 1.69592000 & -1.10711900 & 4.47942100 \\ \mathrm{C} & 2.78863300 & 1.53599600 & -0.55871000 \\ \mathrm{C} & 4.11217100 & 1.94836000 & -0.74578100 \\ \mathrm{C} & 1.82094100 & 1.84251300 & -1.52338900 \\ \mathrm{C} & 4.46085200 & 2.67391000 & -1.87703900 \\ \mathrm{H} & 4.85775500 & 1.70655600 & 0.00740000 \\ \mathrm{C} & 2.18334600 & 2.55291700 & -2.65647900 \\ \mathrm{H} & 0.78897400 & 1.52794200 & -1.39255300 \\ \mathrm{C} & 3.49941300 & 2.97283300 & -2.83620400 \\ \mathrm{H} & 5.48850700 & 3.00255900 & -2.00917500 \\ \mathrm{H} & 1.42762000 & 2.78783600 & -3.40175900 \\ \mathrm{H} & 3.77434800 & 3.53468900 & -3.72576600 \\ \mathrm{O} & 3.11323500 & -0.46534000 & 2.44788100 \\ \mathrm{C} & 2.47738200 & 0.72911300 & 0.62897600 \\ \mathrm{H} & 1.36647100 & -0.04037500 & 0.31661600\end{array}$

(cx)-E-(c) (-1992.837288 a.u.)

$\begin{array}{lrrr}\text { C } & 0.34736400 & 0.61035300 & 0.56108700 \\ \text { C } & 0.45659900 & 1.91370500 & -0.10318300 \\ \text { O } & 0.06162200 & 2.92974900 & 0.69501300 \\ \text { C } & 0.03150800 & 4.19761600 & 0.06191500 \\ \text { H } & -0.66719400 & 4.19150800 & -0.78079600 \\ \text { H } & 1.02556300 & 4.47196800 & -0.31230700 \\ \text { H } & -0.29864200 & 4.90933300 & 0.82090200 \\ \text { O } & 0.86154400 & 2.09076900 & -1.23970700 \\ \text { N } & 3.40804400 & -1.23096100 & -0.08799100 \\ \text { C } & 4.79712900 & -1.56166800 & -0.09059800 \\ \text { C } & 7.46628500 & -2.24017500 & -0.10662800 \\ \text { C } & 5.24188000 & -2.52148600 & -0.99301300 \\ \text { C } & 5.65293800 & -0.94588500 & 0.81653700 \\ \text { C } & 6.58976400 & -2.85680200 & -0.99501200 \\ \text { C } & 6.99824100 & -1.28978800 & 0.79687600 \\ \text { H } & 4.53607700 & -2.99269500 & -1.67097700 \\ \text { H } & 5.26806800 & -0.21304300 & 1.52130100 \\ \text { H } & 6.95548100 & -3.60344300 & -1.69452000 \\ & & & \\ & & 5121 & \end{array}$




\begin{tabular}{|c|c|c|c|}
\hline $\mathrm{H}$ & 7.68076900 & -0.81872100 & 1.49885200 \\
\hline C & 1.31052700 & -1.81113800 & 0.47152900 \\
\hline $\mathrm{H}$ & 0.51885000 & -2.42399100 & 0.03693000 \\
\hline $\mathrm{H}$ & 8.52002700 & -2.50644400 & -0.11344400 \\
\hline $\mathrm{Rh}$ & -1.71461700 & 0.01062900 & 0.15110600 \\
\hline $\mathrm{O}$ & -1.53295300 & -1.80872200 & 1.15205300 \\
\hline C & -2.55379700 & -2.55683300 & 1.22726100 \\
\hline $\mathrm{O}$ & -3.69807400 & -2.37695900 & 0.75594700 \\
\hline $\mathrm{Rh}$ & -4.07009300 & -0.62802700 & -0.29020100 \\
\hline $\mathrm{O}$ & -3.36757400 & -1.50769000 & -2.03858600 \\
\hline C & -2.14378900 & -1.45198700 & -2.29906800 \\
\hline $\mathrm{O}$ & -1.20633000 & -0.92980700 & -1.62327900 \\
\hline $\mathrm{H}$ & -1.82545100 & -1.92431500 & -3.24547200 \\
\hline $\mathrm{O}$ & -4.52205700 & 0.32592800 & 1.49457800 \\
\hline C & -3.58991600 & 0.84137600 & 2.15918000 \\
\hline $\mathrm{O}$ & -2.35176500 & 0.88246300 & 1.90202600 \\
\hline $\mathrm{H}$ & -3.88276600 & 1.32977200 & 3.10537100 \\
\hline $\mathrm{O}$ & -4.28255600 & 1.15815400 & -1.29723600 \\
\hline C & -3.28693800 & 1.91984200 & -1.35421700 \\
\hline $\mathrm{O}$ & -2.12957900 & 1.75420900 & -0.87611500 \\
\hline $\mathrm{H}$ & -3.43310500 & 2.87086300 & -1.89636800 \\
\hline $\mathrm{H}$ & -2.40043900 & -3.49766000 & 1.78645700 \\
\hline C & 1.29376200 & -0.38341500 & -0.06174800 \\
\hline $\mathrm{H}$ & 0.38723300 & 0.67655500 & 1.65416500 \\
\hline C & 1.38334200 & -1.93517400 & 1.97307000 \\
\hline $\mathrm{H}$ & 2.18555100 & -1.30945800 & 2.39053500 \\
\hline $\mathrm{H}$ & 0.43074800 & -1.62313600 & 2.40792800 \\
\hline $\mathrm{H}$ & 1.57197200 & -2.97488000 & 2.25627400 \\
\hline C & 3.46729300 & 1.18994500 & -0.05543200 \\
\hline C & 3.22505400 & 2.09278400 & 0.98447000 \\
\hline C & 4.34249200 & 1.52162100 & -1.09383600 \\
\hline C & 3.86296300 & 3.32638900 & 0.97993900 \\
\hline $\mathrm{H}$ & 2.53568300 & 1.82933300 & 1.78391100 \\
\hline C & 4.95606000 & 2.76502200 & -1.09957100 \\
\hline $\mathrm{H}$ & 4.51165400 & 0.81704200 & -1.90546800 \\
\hline C & 4.71922600 & 3.66541500 & -0.06344900 \\
\hline $\mathrm{H}$ & 3.67652300 & 4.02922900 & 1.78762800 \\
\hline $\mathrm{H}$ & 5.61787400 & 3.03562100 & -1.91787600 \\
\hline $\mathrm{H}$ & 5.20303500 & 4.63898000 & -0.07213200 \\
\hline $\mathrm{O}$ & 2.56873800 & -2.32741000 & -0.10049800 \\
\hline C & 2.77701900 & -0.09436500 & -0.05181300 \\
\hline $\mathrm{H}$ & 1.05996000 & -0.41035800 & -1.14504300 \\
\hline
\end{tabular}

TS(cx)-VIII-(c) (-1992.76249 a.u.) 


\begin{tabular}{|c|c|c|c|}
\hline C & -0.81103900 & -1.36192700 & 1.16533800 \\
\hline C & -1.08849300 & -2.37509200 & 0.13530000 \\
\hline $\mathrm{O}$ & -0.80658600 & -3.60756900 & 0.58798500 \\
\hline C & -0.91333300 & -4.63218100 & -0.39452000 \\
\hline $\mathrm{H}$ & -0.20647000 & -4.44889400 & -1.21036000 \\
\hline $\mathrm{H}$ & -1.92678800 & -4.66763600 & -0.80896600 \\
\hline $\mathrm{H}$ & -0.67488200 & -5.56643100 & 0.11477400 \\
\hline $\mathrm{O}$ & -1.52236700 & -2.14771200 & -0.97454600 \\
\hline $\mathrm{N}$ & -3.12676100 & 1.55722600 & 1.02178000 \\
\hline C & -2.51111000 & 2.47577500 & 0.10535900 \\
\hline C & -1.28413900 & 4.32695400 & -1.57761200 \\
\hline C & -1.99793600 & 2.06300600 & -1.12541900 \\
\hline C & -2.41396900 & 3.81343100 & 0.49442900 \\
\hline C & -1.39603400 & 2.99723100 & -1.96440900 \\
\hline C & -1.79215600 & 4.72867300 & -0.34175200 \\
\hline $\mathrm{H}$ & -2.03312300 & 1.01771100 & -1.43032700 \\
\hline $\mathrm{H}$ & -2.80980100 & 4.10911900 & 1.46177000 \\
\hline $\mathrm{H}$ & -0.99344800 & 2.66776600 & -2.91986900 \\
\hline $\mathrm{H}$ & -1.70888000 & 5.76726900 & -0.02982100 \\
\hline C & -1.45079300 & 0.81159800 & 2.42134600 \\
\hline $\mathrm{H}$ & -0.51085200 & 1.34594400 & 2.22737800 \\
\hline $\mathrm{H}$ & -0.80343100 & 5.04945700 & -2.23265500 \\
\hline $\mathrm{Rh}$ & 1.15593100 & -0.40666000 & 0.22949700 \\
\hline 0 & 1.92133100 & -0.49857700 & 2.14247900 \\
\hline C & 3.11874400 & -0.12007600 & 2.31002400 \\
\hline $\mathrm{O}$ & 3.94107000 & 0.27621900 & 1.44924500 \\
\hline $\mathrm{Rh}$ & 3.37451500 & 0.28513700 & -0.53865800 \\
\hline $\mathrm{O}$ & 2.77077100 & 2.24700300 & -0.38376600 \\
\hline C & 1.62963200 & 2.46199800 & 0.09162700 \\
\hline $\mathrm{O}$ & 0.77736900 & 1.61581500 & 0.49019500 \\
\hline $\mathrm{H}$ & 1.31764000 & 3.51598600 & 0.18122600 \\
\hline $\mathrm{O}$ & 3.84803100 & -1.71357800 & -0.67922300 \\
\hline C & 2.94126500 & -2.55466300 & -0.46456800 \\
\hline $\mathrm{O}$ & 1.74352500 & -2.35060600 & -0.11277300 \\
\hline $\mathrm{H}$ & 3.21674000 & -3.61416200 & -0.59681200 \\
\hline $\mathrm{O}$ & 2.65056000 & 0.24850000 & -2.46176200 \\
\hline C & 1.42123400 & 0.03045600 & -2.61286100 \\
\hline $\mathrm{O}$ & 0.55211300 & -0.23084400 & -1.73480300 \\
\hline $\mathrm{H}$ & 1.04425200 & 0.07001300 & -3.64845100 \\
\hline $\mathrm{H}$ & 3.47693900 & -0.14507100 & 3.35257200 \\
\hline C & -1.74607500 & -0.21589200 & 1.33939100 \\
\hline $\mathrm{H}$ & -0.28823000 & -1.69820600 & 2.06313300 \\
\hline C & -1.47148700 & 0.22739400 & 3.81288700 \\
\hline $\mathrm{H}$ & -2.36948900 & -0.38425100 & 3.95845700 \\
\hline
\end{tabular}




$\begin{array}{cccc}\mathrm{H} & -0.57983300 & -0.38370700 & 3.99050200 \\ \mathrm{H} & -1.48064700 & 1.03657100 & 4.54993400 \\ \mathrm{C} & -3.91702900 & -0.52551900 & -0.06562200 \\ \mathrm{C} & -4.08767400 & -1.91155100 & 0.08676100 \\ \mathrm{C} & -4.79735800 & 0.17299400 & -0.91016000 \\ \mathrm{C} & -5.07894900 & -2.58161000 & -0.61210600 \\ \mathrm{H} & -3.46862400 & -2.45830000 & 0.79396100 \\ \mathrm{C} & -5.77628700 & -0.50356800 & -1.61823500 \\ \mathrm{H} & -4.70413900 & 1.25195700 & -1.00603000 \\ \mathrm{C} & -5.91511200 & -1.88341900 & -1.47823600 \\ \mathrm{H} & -5.20217700 & -3.65323300 & -0.47644900 \\ \mathrm{H} & -6.44161700 & 0.04763900 & -2.27786200 \\ \mathrm{H} & -6.68688900 & -2.41140200 & -2.03328600 \\ \mathrm{O} & -2.57098600 & 1.71529200 & 2.31793100 \\ \mathrm{C} & -2.94368700 & 0.18074300 & 0.73221500 \\ \mathrm{H} & -0.89696400 & -0.06026600 & 0.43425500\end{array}$

TS(cx)-VI-(o) (-1992.712857 a.u.)

$\begin{array}{lccc}\text { C } & -2.10017000 & -1.86713200 & 0.74178600 \\ \mathrm{C} & -1.10712000 & -2.01737200 & -0.30761900 \\ \mathrm{O} & -0.85381100 & -3.30233300 & -0.55209300 \\ \mathrm{C} & 0.04852500 & -3.58590600 & -1.62697100 \\ \mathrm{H} & 1.07775800 & -3.39533200 & -1.31403300 \\ \mathrm{H} & -0.18406200 & -2.96670600 & -2.49770500 \\ \mathrm{H} & -0.09193500 & -4.64335100 & -1.85392300 \\ \mathrm{O} & -0.55049600 & -1.10090600 & -0.93072900 \\ \mathrm{~N} & -3.39335200 & 1.44784100 & 0.52208000 \\ \mathrm{C} & -2.52946400 & 2.24688100 & -0.27309800 \\ \mathrm{C} & -1.02100400 & 3.93342200 & -1.90612900 \\ \mathrm{C} & -2.33515800 & 1.92763500 & -1.61819600 \\ \mathrm{C} & -1.96582000 & 3.40916200 & 0.25753700 \\ \mathrm{C} & -1.58495100 & 2.77203300 & -2.42564900 \\ \mathrm{C} & -1.21242000 & 4.24094400 & -0.56121600 \\ \mathrm{H} & -2.76580000 & 1.01685800 & -2.03024700 \\ \mathrm{H} & -2.13249600 & 3.64933300 & 1.30309000 \\ \mathrm{H} & -1.43247600 & 2.50990900 & -3.46994500 \\ \mathrm{H} & -0.77838400 & 5.14664400 & -0.14158800 \\ \mathrm{C} & -2.13855800 & 0.44330000 & 2.18003500 \\ \mathrm{H} & -1.11183500 & 0.75791100 & 1.91481100 \\ \mathrm{H} & -0.44045100 & 4.59555800 & -2.54497700 \\ \mathrm{Rh} & 1.52356600 & -0.50150100 & -0.25968300 \\ \mathrm{O} & 2.14154300 & -2.36359800 & 0.40908400 \\ \mathrm{C} & 3.30476400 & -2.45772300 & 0.89371900 \\ \mathrm{O} & 4.17362100 & -1.55668000 & 1.01331400 \\ & & & \\ & & 5124 & \\ & & & \\ & & & \\ & & & \end{array}$




$\begin{array}{lccc}\text { Rh } & 3.69761300 & 0.33837200 & 0.36263000 \\ \mathrm{O} & 2.92759700 & 0.77158800 & 2.22340400 \\ \mathrm{C} & 1.73061400 & 0.46615800 & 2.44820900 \\ \mathrm{O} & 0.90149600 & -0.08146300 & 1.66557100 \\ \mathrm{H} & 1.34867400 & 0.70446500 & 3.45652000 \\ \mathrm{O} & 4.33533300 & -0.14093500 & -1.52913500 \\ \mathrm{C} & 3.49986800 & -0.63872700 & -2.32879600 \\ \mathrm{O} & 2.28246900 & -0.90444600 & -2.12470600 \\ \mathrm{H} & 3.87770400 & -0.87625100 & -3.33681900 \\ \mathrm{O} & 3.09014500 & 2.18113000 & -0.30938000 \\ \mathrm{C} & 1.91301900 & 2.28583200 & -0.75121300 \\ \mathrm{O} & 1.03714900 & 1.38479100 & -0.85662600 \\ \mathrm{H} & 1.59805700 & 3.28766800 & -1.08854500 \\ \mathrm{H} & 3.59115000 & -3.45863200 & 1.25700300 \\ \mathrm{C} & -2.54675100 & -0.57697200 & 1.17544600 \\ \mathrm{H} & -2.12994800 & -2.72674400 & 1.41655600 \\ \mathrm{C} & -2.23183100 & 0.03293000 & 3.62749500 \\ \mathrm{H} & -3.24527100 & -0.30920900 & 3.86479500 \\ \mathrm{H} & -1.52242900 & -0.77890900 & 3.82567800 \\ \mathrm{H} & -1.98697700 & 0.87661700 & 4.27994500 \\ \mathrm{C} & -4.77974500 & -0.51754600 & -0.11445200 \\ \mathrm{C} & -5.82495700 & -0.26067800 & 0.77986400 \\ \mathrm{C} & -5.03895100 & -1.19639000 & -1.30122400 \\ \mathrm{C} & -7.10565000 & -0.71112300 & 0.49593000 \\ \mathrm{H} & -5.61378200 & 0.29248900 & 1.69344200 \\ \mathrm{C} & -6.32598600 & -1.64723000 & -1.58375700 \\ \mathrm{H} & -4.23117600 & -1.36784200 & -2.01269100 \\ \mathrm{C} & -7.35777300 & -1.40854300 & -0.68494400 \\ \mathrm{H} & -7.91237200 & -0.52148800 & 1.20043000 \\ \mathrm{H} & -6.51961300 & -2.17889000 & -2.51212400 \\ \mathrm{H} & -8.36334600 & -1.75951100 & -0.90444100 \\ \mathrm{O} & -3.06551600 & 1.50700400 & 1.91140900 \\ \mathrm{C} & -31847400 & 0.00933800 & 0.25977700 \\ \mathrm{H} & -1.09564200 & -0.41248000\end{array}$

TS(cx)-VII-(o) (-1992.781071 a.u.)

$\begin{array}{lccc}\text { C } & -1.25241100 & 2.99288600 & -0.85556200 \\ \text { C } & -0.09376100 & 2.95813800 & -0.07634000 \\ \text { O } & 0.69054000 & 4.04286400 & -0.22637700 \\ \text { C } & 1.93622500 & 4.02690300 & 0.45679800 \\ \text { H } & 2.61616000 & 3.29417800 & 0.00961200 \\ \text { H } & 1.80731000 & 3.77547300 & 1.51426200 \\ \text { H } & 2.34653700 & 5.03313000 & 0.35200100 \\ \text { O } & 0.22513100 & 2.03392400 & 0.71854900\end{array}$




\begin{tabular}{|c|c|c|c|}
\hline N & -3.87044200 & 0.36970200 & -0.64984000 \\
\hline C & -4.11785100 & -1.04028000 & -0.60031700 \\
\hline C & -4.51095300 & -3.76975300 & -0.37563500 \\
\hline C & -4.95371000 & -1.52864300 & 0.39510600 \\
\hline C & -3.50469700 & -1.89603000 & -1.51201900 \\
\hline C & -5.13820300 & -2.90238000 & 0.51340600 \\
\hline C & -3.70432800 & -3.26573800 & -1.39487100 \\
\hline $\mathrm{H}$ & -5.42401000 & -0.82719300 & 1.07991800 \\
\hline $\mathrm{H}$ & -2.88869000 & -1.47470300 & -2.30377000 \\
\hline $\mathrm{H}$ & -5.77768300 & -3.29489700 & 1.29985500 \\
\hline $\mathrm{H}$ & -3.22675500 & -3.94367000 & -2.09885200 \\
\hline C & -3.28495500 & 2.07826500 & -2.01141900 \\
\hline $\mathrm{H}$ & -2.84940000 & 2.19906100 & -3.01029700 \\
\hline $\mathrm{H}$ & -4.65824400 & -4.84287700 & -0.28251800 \\
\hline $\mathrm{Rh}$ & 1.50239400 & 0.36049900 & 0.10925600 \\
\hline O & 2.08008500 & 1.24965600 & -1.65716300 \\
\hline C & 2.85551900 & 0.59441400 & -2.40959700 \\
\hline 0 & 3.34010900 & -0.55203700 & -2.22645900 \\
\hline $\mathrm{Rh}$ & 2.86069000 & -1.55653700 & -0.48936400 \\
\hline $\mathrm{O}$ & 1.19912800 & -2.36694800 & -1.40297200 \\
\hline C & 0.13436900 & -1.70596900 & -1.39074800 \\
\hline 0 & -0.07532700 & -0.56176800 & -0.89118100 \\
\hline $\mathrm{H}$ & -0.73782600 & -2.18230500 & -1.87265300 \\
\hline O & 4.43245900 & -0.65351500 & 0.47112500 \\
\hline C & 4.22397200 & 0.45912400 & 1.02010200 \\
\hline 0 & 3.15526000 & 1.13094400 & 1.05311500 \\
\hline $\mathrm{H}$ & 5.08439400 & 0.91137200 & 1.54029000 \\
\hline 0 & 2.27913600 & -2.42291400 & 1.28586400 \\
\hline C & 1.50335700 & -1.76604300 & 2.03061000 \\
\hline O & 1.01210200 & -0.62088000 & 1.84120600 \\
\hline $\mathrm{H}$ & 1.21099500 & -2.25646300 & 2.97530000 \\
\hline $\mathrm{H}$ & 3.14182900 & 1.09719300 & -3.34827600 \\
\hline C & -2.22270700 & 1.95574500 & -0.93779000 \\
\hline $\mathrm{H}$ & -1.32085000 & 3.77177300 & -1.61007000 \\
\hline C & -4.27109800 & 3.18351800 & -1.69051400 \\
\hline $\mathrm{H}$ & -4.67019700 & 3.05687100 & -0.67733500 \\
\hline $\mathrm{H}$ & -3.78958200 & 4.16428700 & -1.74976400 \\
\hline $\mathrm{H}$ & -5.10479400 & 3.15476800 & -2.39844600 \\
\hline C & -2.34852400 & 0.60807600 & 1.30879600 \\
\hline C & -2.20589400 & 1.67084700 & 2.20524100 \\
\hline C & -2.17791500 & -0.70560500 & 1.75892600 \\
\hline C & -1.93766400 & 1.42316000 & 3.54347500 \\
\hline $\mathrm{H}$ & -2.30824200 & 2.69029300 & 1.83773900 \\
\hline C & -1.90616500 & -0.94722400 & 3.09762600 \\
\hline
\end{tabular}




$\begin{array}{lrrr}\mathrm{H} & -2.22910100 & -1.53596400 & 1.05859000 \\ \mathrm{C} & -1.79299800 & 0.11339100 & 3.99105800 \\ \mathrm{H} & -1.83114200 & 2.25385300 & 4.23616300 \\ \mathrm{H} & -1.76977600 & -1.96997400 & 3.44175300 \\ \mathrm{H} & -1.57739300 & -0.08054500 & 5.03931700 \\ \mathrm{O} & -3.91255500 & 0.79722300 & -2.00432800 \\ \mathrm{C} & -2.73244200 & 0.93404600 & -0.06883400 \\ \mathrm{H} & -1.68083400 & 0.80268800 & -1.05510600\end{array}$

(cx)-E-(o) (-1992.809884 a.u.)

$\begin{array}{lccc}\mathrm{C} & -1.08199600 & 2.43441200 & -1.77190800 \\ \mathrm{C} & -0.13276900 & 2.85225600 & -0.86454800 \\ \mathrm{O} & 0.18511300 & 4.17456900 & -0.90326600 \\ \mathrm{C} & 1.11056200 & 4.61261100 & 0.07413700 \\ \mathrm{H} & 2.05137100 & 4.05368200 & 0.02142300 \\ \mathrm{H} & 0.70400700 & 4.49963700 & 1.08724700 \\ \mathrm{H} & 1.28969800 & 5.67006700 & -0.13637800 \\ \mathrm{O} & 0.39547000 & 2.14397100 & 0.05555700 \\ \mathrm{~N} & -3.09980900 & -0.43804500 & -0.96378400 \\ \mathrm{C} & -4.01350800 & -1.30825400 & -0.30291700 \\ \mathrm{C} & -5.74672100 & -3.01875600 & 0.99203000 \\ \mathrm{C} & -5.02922700 & -0.77268300 & 0.48246800 \\ \mathrm{C} & -3.85829200 & -2.68092200 & -0.46952600 \\ \mathrm{C} & -5.89272900 & -1.64154600 & 1.13630200 \\ \mathrm{C} & -4.73536100 & -3.53512100 & 0.18734300 \\ \mathrm{H} & -5.13290600 & 0.30456600 & 0.58072800 \\ \mathrm{H} & -3.06740200 & -3.05958300 & -1.11071400 \\ \mathrm{H} & -6.68959100 & -1.23757100 & 1.75478100 \\ \mathrm{H} & -4.62555400 & -4.60969000 & 0.06862200 \\ \mathrm{C} & -2.14933900 & 0.33285400 & -2.87039100 \\ \mathrm{H} & -1.39537800 & -0.07532700 & -3.55080000 \\ \mathrm{H} & -6.42859800 & -3.69219900 & 1.50471200 \\ \mathrm{Rh} & 1.51163200 & 0.29624400 & 0.01271300 \\ \mathrm{O} & 1.55145400 & 0.05223600 & -2.04354600 \\ \mathrm{C} & 2.23932200 & -0.90104000 & -2.50818200 \\ \mathrm{O} & 2.91099800 & -1.76140200 & -1.88531800 \\ \mathrm{Rh} & 2.95819000 & -1.65589200 & 0.17564200 \\ \mathrm{O} & 1.26611300 & -2.84020700 & 0.28719900 \\ \mathrm{C} & 0.15102700 & -2.27043500 & 0.25574600 \\ \mathrm{O} & -0.10471100 & -1.03656100 & 0.14771600 \\ \mathrm{H} & -0.73424100 & -2.92937500 & 0.33675000 \\ \mathrm{O} & 4.55229100 & -0.36658800 & 0.06030600 \\ \mathrm{C} & 4.31499000 & 0.86454700 & -0.04983700 \\ \mathrm{O} & 3.19773600 & 1.44982500 & -0.09844100 \\ & & 5127 & \\ & & & \\ & & & \\ & & & \\ & & & \end{array}$




$\begin{array}{lrrr}\mathrm{H} & 5.19663800 & 1.52385500 & -0.11254200 \\ \mathrm{O} & 2.90596000 & -1.40812300 & 2.21985400 \\ \mathrm{C} & 2.23159000 & -0.45171700 & 2.68575200 \\ \mathrm{O} & 1.54971300 & 0.40960100 & 2.06635700 \\ \mathrm{H} & 2.23344700 & -0.35275700 & 3.78480900 \\ \mathrm{H} & 2.24535700 & -0.98568900 & -3.60844700 \\ \mathrm{C} & -1.49944000 & 1.02191100 & -1.68147100 \\ \mathrm{H} & -1.57172400 & 3.14417500 & -2.43005700 \\ \mathrm{C} & -3.20140200 & 1.13449100 & -3.59488700 \\ \mathrm{H} & -3.93092700 & 1.55677000 & -2.89158100 \\ \mathrm{H} & -2.72933200 & 1.96559400 & -4.12797000 \\ \mathrm{H} & -3.73297800 & 0.51139700 & -4.31989900 \\ \mathrm{C} & -2.49001800 & 1.18496200 & 0.76386100 \\ \mathrm{C} & -2.68743700 & 2.56522900 & 0.88557300 \\ \mathrm{C} & -2.23862600 & 0.40006900 & 1.89635200 \\ \mathrm{C} & -2.62724700 & 3.15433100 & 2.14178300 \\ \mathrm{H} & -2.85072500 & 3.16283600 & -0.00696900 \\ \mathrm{C} & -2.16021300 & 1.00405000 & 3.14019400 \\ \mathrm{H} & -2.04578200 & -0.66350600 & 1.78086800 \\ \mathrm{C} & -2.35247900 & 2.37910800 & 3.26323600 \\ \mathrm{H} & -2.77718600 & 4.22628000 & 2.24043900 \\ \mathrm{H} & -1.93079600 & 0.40324500 & 4.01623100 \\ \mathrm{H} & -2.28311800 & 2.84810800 & 4.24189300 \\ \mathrm{O} & -2.77345400 & -0.84400400 & -2.25494400 \\ \mathrm{C} & -2.43565300 & 0.60445000 & -0.56635500 \\ \mathrm{H} & -0.62128200 & 0.41057800 & -1.41293800 \\ & & & \end{array}$

TS(cx)-VIII-(o) (-1992.767782 a.u.)

\begin{tabular}{|c|c|c|c|}
\hline C & 0.96531700 & 2.49090500 & 1.79380200 \\
\hline C & 0.14435000 & 2.88351900 & 0.71705400 \\
\hline $\mathrm{O}$ & -0.37007200 & 4.11887700 & 0.84313900 \\
\hline C & -1.25286500 & 4.52631300 & -0.19814300 \\
\hline $\mathrm{H}$ & -2.09337900 & 3.83201400 & -0.29368500 \\
\hline $\mathrm{H}$ & -0.72397200 & 4.57431900 & -1.15610500 \\
\hline $\mathrm{H}$ & -1.60429800 & 5.51970400 & 0.08637400 \\
\hline $\mathrm{O}$ & -0.11474300 & 2.18663600 & -0.29764400 \\
\hline $\mathrm{N}$ & 3.01555400 & -0.49391900 & 1.01455700 \\
\hline C & 3.49895800 & -1.59927100 & 0.29435100 \\
\hline C & 4.45383400 & -3.79402600 & -1.12891300 \\
\hline C & 4.35003000 & -1.40277600 & -0.79636900 \\
\hline C & 3.15876600 & -2.89181400 & 0.70155000 \\
\hline C & 4.80645900 & -2.50224700 & -1.51023400 \\
\hline C & 3.63997100 & -3.98063500 & -0.01579600 \\
\hline $\mathrm{H}$ & 4.64938600 & -0.39723600 & -1.07693900 \\
\hline
\end{tabular}




\begin{tabular}{|c|c|c|c|}
\hline $\mathrm{H}$ & 2.52376100 & -3.02931300 & 1.57105200 \\
\hline $\mathrm{H}$ & 5.46119300 & -2.34409700 & -2.36374100 \\
\hline $\mathrm{H}$ & 3.37050800 & -4.98535300 & 0.30153400 \\
\hline C & 2.24879500 & 0.45849800 & 2.91959200 \\
\hline $\mathrm{H}$ & 1.43448700 & 0.23528700 & 3.62357600 \\
\hline $\mathrm{H}$ & 4.82330200 & -4.65011000 & -1.68735100 \\
\hline $\mathrm{Rh}$ & -1.32772300 & 0.32673800 & -0.13280800 \\
\hline $\mathrm{O}$ & -1.15358200 & 0.14850400 & 1.92656700 \\
\hline C & -1.79163000 & -0.78351200 & 2.50123700 \\
\hline $\mathrm{O}$ & -2.57049700 & -1.61903700 & 1.98534000 \\
\hline $\mathrm{Rh}$ & -2.87081500 & -1.54209300 & -0.05742700 \\
\hline $\mathrm{O}$ & -1.27849600 & -2.81312200 & -0.32382100 \\
\hline C & -0.13149100 & -2.30218900 & -0.38282300 \\
\hline $\mathrm{O}$ & 0.18483300 & -1.07973900 & -0.32116300 \\
\hline $\mathrm{H}$ & 0.71375400 & -3.00288800 & -0.50134800 \\
\hline $\mathrm{O}$ & -4.37998000 & -0.17023600 & 0.21147500 \\
\hline C & -4.08059100 & 1.05113400 & 0.22681700 \\
\hline $\mathrm{O}$ & -2.94014700 & 1.58046400 & 0.10252600 \\
\hline $\mathrm{H}$ & -4.91821100 & 1.75412400 & 0.36686400 \\
\hline $\mathrm{O}$ & -3.05632600 & -1.34657800 & -2.09399200 \\
\hline C & -2.37401800 & -0.46040700 & -2.67330500 \\
\hline $\mathrm{O}$ & -1.56809000 & 0.36859200 & -2.16682800 \\
\hline $\mathrm{H}$ & -2.49339900 & -0.39799200 & -3.76750700 \\
\hline $\mathrm{H}$ & -1.64083200 & -0.85987100 & 3.59094300 \\
\hline C & 1.77726800 & 1.23837600 & 1.71509200 \\
\hline $\mathrm{H}$ & 0.96826400 & 3.06471900 & 2.71595900 \\
\hline C & 3.43526200 & 1.07986800 & 3.62539500 \\
\hline $\mathrm{H}$ & 4.24200900 & 1.27421000 & 2.90856400 \\
\hline $\mathrm{H}$ & 3.14475600 & 2.03447700 & 4.07921000 \\
\hline $\mathrm{H}$ & 3.81482300 & 0.42080700 & 4.41229600 \\
\hline C & 2.59151800 & 1.28232700 & -0.70670200 \\
\hline C & 3.11357700 & 2.57860200 & -0.75365600 \\
\hline C & 2.18012600 & 0.65905700 & -1.88717500 \\
\hline C & 3.23014000 & 3.24276800 & -1.96694000 \\
\hline $\mathrm{H}$ & 3.41393900 & 3.06466600 & 0.17366000 \\
\hline C & 2.28725600 & 1.33269400 & -3.09653000 \\
\hline $\mathrm{H}$ & 1.74236300 & -0.33298000 & -1.84274200 \\
\hline C & 2.81077800 & 2.62119400 & -3.14010000 \\
\hline $\mathrm{H}$ & 3.64185000 & 4.24868500 & -1.99636800 \\
\hline $\mathrm{H}$ & 1.94716900 & 0.85031900 & -4.00951000 \\
\hline $\mathrm{H}$ & 2.88852200 & 3.14474200 & -4.09027600 \\
\hline $\mathrm{O}$ & 2.60961800 & -0.80421400 & 2.32282000 \\
\hline C & 2.44637600 & 0.67826000 & 0.62230500 \\
\hline $\mathrm{H}$ & 0.57005400 & 1.12918900 & 1.74382700 \\
\hline
\end{tabular}


(t)-C-2Rh ${ }_{2} \mathrm{~L}_{4}$ (-2970.540905 a.u)

\begin{tabular}{|c|c|c|c|}
\hline C & -1.13501700 & -0.14465600 & 0.36302500 \\
\hline C & -0.68406200 & -1.53544100 & 0.33089200 \\
\hline $\mathrm{O}$ & -0.71843100 & -2.07820600 & 1.57475800 \\
\hline C & -0.46392400 & -3.47450000 & 1.62431400 \\
\hline $\mathrm{H}$ & 0.57136700 & -3.69640900 & 1.33051300 \\
\hline $\mathrm{H}$ & -1.14552700 & -4.01439800 & 0.96040500 \\
\hline $\mathrm{H}$ & -0.62636900 & -3.77489200 & 2.66101500 \\
\hline $\mathrm{O}$ & -0.35362900 & -2.19759300 & -0.63979100 \\
\hline $\mathrm{O}$ & 1.63861900 & 1.01333300 & -1.67872200 \\
\hline$N$ & 1.46963300 & 2.05356200 & -0.76938300 \\
\hline C & 2.33981200 & 3.16775100 & -0.98445000 \\
\hline C & 3.97659100 & 5.33896100 & -1.48720600 \\
\hline C & 1.88121400 & 4.45183900 & -0.69098300 \\
\hline C & 3.58977000 & 2.95620700 & -1.56016400 \\
\hline C & 2.71348700 & 5.53429800 & -0.93400300 \\
\hline C & 4.40373600 & 4.05490000 & -1.80694100 \\
\hline $\mathrm{H}$ & 0.88559800 & 4.60268500 & -0.28322200 \\
\hline $\mathrm{H}$ & 3.92473100 & 1.95481300 & -1.81287100 \\
\hline $\mathrm{H}$ & 2.36249700 & 6.53726800 & -0.70700100 \\
\hline $\mathrm{H}$ & 5.38074700 & 3.89820600 & -2.25580100 \\
\hline C & 0.27767500 & 0.40445600 & -1.84658000 \\
\hline $\mathrm{H}$ & 0.44773400 & -0.67458400 & -1.85727400 \\
\hline $\mathrm{H}$ & 4.62161200 & 6.19172200 & -1.68238900 \\
\hline $\mathrm{Rh}$ & -3.26184500 & -0.26033500 & -0.00553800 \\
\hline 0 & -3.02289500 & -0.64774500 & -2.02182900 \\
\hline C & -4.07095800 & -0.82890400 & -2.71195000 \\
\hline $\mathrm{O}$ & -5.27118000 & -0.77253000 & -2.35708300 \\
\hline $\mathrm{Rh}$ & -5.71591000 & -0.31367900 & -0.38035000 \\
\hline $\mathrm{O}$ & -5.58750600 & 1.71515900 & -0.82467400 \\
\hline C & -4.47825300 & 2.28118200 & -0.75534100 \\
\hline $\mathrm{O}$ & -3.34869200 & 1.78463000 & -0.44308600 \\
\hline $\mathrm{H}$ & -4.46055700 & 3.35986700 & -0.99414000 \\
\hline $\mathrm{O}$ & -5.63718900 & -2.32215600 & 0.08265800 \\
\hline C & -4.52008300 & -2.83023800 & 0.34277500 \\
\hline $\mathrm{O}$ & -3.38266500 & -2.27894200 & 0.38627200 \\
\hline $\mathrm{H}$ & -4.51884500 & -3.91113300 & 0.56803500 \\
\hline $\mathrm{O}$ & -5.89524900 & 0.13675900 & 1.64013200 \\
\hline C & -4.85636500 & 0.25587100 & 2.33210200 \\
\hline $\mathrm{O}$ & -3.64347200 & 0.16251500 & 1.97885000 \\
\hline $\mathrm{H}$ & -5.01023600 & 0.47801400 & 3.40344700 \\
\hline
\end{tabular}




$\begin{array}{lrrr}\text { H } & -3.88928100 & -1.06516700 & -3.77576500 \\ \mathrm{C} & -0.49400400 & 0.85906400 & -0.59342400 \\ \mathrm{H} & -1.31427200 & 1.52465500 & -0.94347100 \\ \mathrm{C} & -0.30400800 & 0.93033900 & -3.12752800 \\ \mathrm{H} & -1.31041300 & 0.50804900 & -3.22989600 \\ \mathrm{H} & -0.38706100 & 2.02554700 & -3.10116100 \\ \mathrm{H} & 0.30006200 & 0.63887100 & -3.99108500 \\ \mathrm{C} & 0.20774900 & 2.57177100 & 1.25057600 \\ \mathrm{C} & 1.30160600 & 2.86935700 & 2.08104900 \\ \mathrm{C} & -1.10122200 & 2.83689000 & 1.67852900 \\ \mathrm{C} & 1.08114000 & 3.44471300 & 3.32072500 \\ \mathrm{H} & 2.30841300 & 2.61096800 & 1.75661700 \\ \mathrm{C} & -1.30458500 & 3.42950900 & 2.91728500 \\ \mathrm{H} & -1.94963500 & 2.58202600 & 1.04343800 \\ \mathrm{C} & -0.22019600 & 3.73132100 & 3.73545600 \\ \mathrm{H} & 1.92261700 & 3.66179900 & 3.97329500 \\ \mathrm{H} & -2.31817800 & 3.63630000 & 3.24963100 \\ \mathrm{H} & -0.38781100 & 4.18100300 & 4.71123800 \\ \mathrm{C} & 0.43085200 & 1.86245400 & 0.00873900 \\ \mathrm{H} & -1.11805800 & 0.23272500 & 1.39049700 \\ \mathrm{Rh} & 4.36833600 & -2.10645300 & 0.72696800 \\ \mathrm{Rh} & 3.20501000 & -0.42713600 & -0.52027800 \\ \mathrm{O} & 3.12967800 & -3.48888300 & -0.14526100 \\ \mathrm{O} & 2.97994800 & -1.87731100 & 2.22511600 \\ \mathrm{O} & 5.68435400 & -2.22523700 & -0.84836500 \\ \mathrm{O} & 5.50821700 & -0.61180100 & 1.56329800 \\ \mathrm{O} & 4.71870800 & -0.46778400 & -1.90994400 \\ \mathrm{O} & 4.22331900 & 0.99356600 & 0.60621000 \\ \mathrm{O} & 2.32031300 & -1.92688400 & -1.56546000 \\ \mathrm{O} & 1.80252300 & -0.41452000 & 0.96540400 \\ \mathrm{C} & 2.40095300 & -3.09836400 & -1.09849700 \\ \mathrm{C} & 2.01069600 & -1.10501700 & 2.00792800 \\ \mathrm{C} & 5.58436100 & -1.38004300 & -1.77568700 \\ \mathrm{C} & 5.15905000 & 0.57660500 & 1.34838400 \\ \mathrm{H} & 5.73915000 & 1.35425000 & 1.87122500 \\ \mathrm{H} & 1.25098500 & -1.01323500 & 2.79867000 \\ \mathrm{H} & -3.85581400 & -1.58667200 \\ & & -1.43881100 & -2.56964500\end{array}$

TS(t)-IV-2Rh ${ }_{2} \mathrm{~L}_{4}$ (-2970.501858 a.u.)

$\begin{array}{llll}C & -1.07412600 & -0.52973800 & 0.43018000 \\ C & -0.77265200 & -1.94533800 & 0.13323000 \\ O & -0.79373500 & -2.66567100 & 1.26897300 \\ C & -0.59187600 & -4.06252300 & 1.08407600 \\ & & \end{array}$




\begin{tabular}{|c|c|c|c|}
\hline $\mathrm{H}$ & 0.40512300 & -4.25730800 & 0.67054600 \\
\hline $\mathrm{H}$ & -1.34263300 & -4.47101000 & 0.40085600 \\
\hline $\mathrm{H}$ & -0.68648900 & -4.51470100 & 2.07222800 \\
\hline $\mathrm{O}$ & -0.52871800 & -2.41908300 & -0.95554400 \\
\hline $\mathrm{O}$ & 1.70946200 & 0.99008200 & -1.47496900 \\
\hline $\mathrm{N}$ & 1.37989100 & 2.02553900 & -0.57419800 \\
\hline C & 1.81728400 & 3.31337100 & -0.94985700 \\
\hline C & 2.70321300 & 5.84037300 & -1.66169600 \\
\hline C & 0.97815800 & 4.41241700 & -0.76435100 \\
\hline C & 3.09022600 & 3.46043800 & -1.50244500 \\
\hline C & 1.43646200 & 5.67741400 & -1.10934200 \\
\hline C & 3.51920700 & 4.73000200 & -1.86491100 \\
\hline $\mathrm{H}$ & -0.02132100 & 4.27234800 & -0.35782200 \\
\hline $\mathrm{H}$ & 3.72170600 & 2.58647000 & -1.64724600 \\
\hline $\mathrm{H}$ & 0.78791500 & 6.53765500 & -0.96367400 \\
\hline $\mathrm{H}$ & 4.50620600 & 4.85220900 & -2.30405200 \\
\hline C & 0.45162200 & 0.24557100 & -1.66717200 \\
\hline $\mathrm{H}$ & 0.75473200 & -0.79053900 & -1.83129400 \\
\hline $\mathrm{H}$ & 3.05169200 & 6.83127300 & -1.94140700 \\
\hline $\mathrm{Rh}$ & -3.31744400 & -0.37065500 & -0.03930400 \\
\hline $\mathrm{O}$ & -3.18017700 & -0.76889700 & -2.05908100 \\
\hline C & -4.25590200 & -0.89442300 & -2.71556100 \\
\hline $\mathrm{O}$ & -5.43843400 & -0.77308100 & -2.31419000 \\
\hline $\mathrm{Rh}$ & -5.75764700 & -0.28881600 & -0.33364100 \\
\hline $\mathrm{O}$ & -5.54316300 & 1.71887000 & -0.79351400 \\
\hline C & -4.40271400 & 2.22893400 & -0.75301900 \\
\hline $\mathrm{O}$ & -3.29764800 & 1.67341200 & -0.46099500 \\
\hline $\mathrm{H}$ & -4.33279400 & 3.30199400 & -0.99816900 \\
\hline $\mathrm{O}$ & -5.79777900 & -2.29249500 & 0.13111100 \\
\hline C & -4.70338800 & -2.87057900 & 0.33981700 \\
\hline $\mathrm{O}$ & -3.53637700 & -2.38269400 & 0.33680200 \\
\hline $\mathrm{H}$ & -4.75642100 & -3.95050900 & 0.55770400 \\
\hline $\mathrm{O}$ & -5.87534000 & 0.17450000 & 1.67910600 \\
\hline C & -4.81557400 & 0.22288400 & 2.34816300 \\
\hline $\mathrm{O}$ & -3.62287300 & 0.04960700 & 1.95810500 \\
\hline $\mathrm{H}$ & -4.92611500 & 0.45013600 & 3.42237100 \\
\hline $\mathrm{H}$ & -4.12552000 & -1.14139500 & -3.78232700 \\
\hline C & -0.22517000 & 0.47600100 & -0.32550900 \\
\hline $\mathrm{H}$ & -1.47669100 & 0.50752000 & -0.42155500 \\
\hline C & -0.31347300 & 0.82102700 & -2.83076600 \\
\hline $\mathrm{H}$ & -1.25834100 & 0.27139600 & -2.92769500 \\
\hline H & -0.54747500 & 1.87977100 & -2.65507200 \\
\hline $\mathrm{H}$ & 0.25447100 & 0.72533900 & -3.76073900 \\
\hline C & 0.10541500 & 2.25567100 & 1.50557900 \\
\hline
\end{tabular}




$\begin{array}{lccc}\mathrm{C} & 1.20134900 & 2.68326000 & 2.27131900 \\ \mathrm{C} & -1.19324800 & 2.38155000 & 2.01223200 \\ \mathrm{C} & 0.98954400 & 3.22347800 & 3.53083300 \\ \mathrm{H} & 2.20863200 & 2.55208500 & 1.87830700 \\ \mathrm{C} & -1.39142100 & 2.92503400 & 3.27491400 \\ \mathrm{H} & -2.04628500 & 2.08094000 & 1.40861800 \\ \mathrm{C} & -0.30440100 & 3.34584000 & 4.03462000 \\ \mathrm{H} & 1.83907400 & 3.54516700 & 4.12831100 \\ \mathrm{H} & -2.40302100 & 3.01814300 & 3.66302700 \\ \mathrm{H} & -0.46353700 & 3.76901300 & 5.02364800 \\ \mathrm{C} & 0.34971800 & 1.62188800 & 0.21690700 \\ \mathrm{H} & -1.13520000 & -0.26867200 & 1.48855200 \\ \mathrm{Rh} & 4.88560500 & -1.81744500 & 0.56429100 \\ \mathrm{Rh} & 3.27774200 & -0.33858200 & -0.44762500 \\ \mathrm{O} & 4.18566600 & -3.30291900 & -0.67127500 \\ \mathrm{O} & 3.45389300 & -2.34266600 & 1.94440100 \\ \mathrm{O} & 6.21119900 & -1.20907700 & -0.88673000 \\ \mathrm{O} & 5.47328100 & -0.24604700 & 1.75489300 \\ \mathrm{O} & 4.72200400 & 0.25080600 & -1.78156100 \\ \mathrm{O} & 3.90782100 & 1.13095100 & 0.86011300 \\ \mathrm{O} & 2.74446800 & -1.87914300 & -1.68590900 \\ \mathrm{O} & 1.92242100 & -0.99890300 & 0.95379400 \\ \mathrm{C} & 3.28841700 & -3.00538800 & -1.50185800 \\ \mathrm{C} & 2.30807500 & -1.84040200 & 1.81613600 \\ \mathrm{C} & 5.84512200 & -0.32567200 & -1.70383300 \\ \mathrm{C} & 4.85801200 & 0.84410400 & 1.64441700 \\ \mathrm{H} & 5.18450700 & 1.65598600 & 2.31476700 \\ \mathrm{H} & 1.53665900 & -2.16705800 & 2.53278500 \\ \mathrm{H} & 2.93067100 & -3.81843600 & -2.15221900 \\ \mathrm{H} & 6.59465900 & -0.01078600 & -2.44739800\end{array}$

(c)-C-2Rh $\mathrm{L}_{4}$ (-2970.536409 a.u)

$\begin{array}{lrrr}\mathrm{C} & -1.52090900 & -0.84675000 & 1.31906700 \\ \mathrm{C} & -1.50406600 & -2.23840500 & 1.80507700 \\ \mathrm{O} & -0.89532300 & -3.08818600 & 0.93869000 \\ \mathrm{C} & -0.98060100 & -4.45976600 & 1.28953900 \\ \mathrm{H} & -2.02703700 & -4.77703800 & 1.34557700 \\ \mathrm{H} & -0.50943200 & -4.65010800 & 2.25991000 \\ \mathrm{H} & -0.46011400 & -5.00856900 & 0.50106900 \\ \mathrm{O} & -1.99753400 & -2.62191400 & 2.84321800 \\ \mathrm{~N} & 0.68490000 & 1.48809300 & -0.34660100 \\ \mathrm{C} & 0.82807700 & 2.77530500 & -0.94918700 \\ \mathrm{C} & 1.03185800 & 5.25257700 & -2.14878600 \\ & & & \end{array}$




\begin{tabular}{|c|c|c|c|}
\hline C & -0.25181200 & 3.65547800 & -0.87979500 \\
\hline C & 1.99727600 & 3.10032500 & -1.62584000 \\
\hline C & -0.13691900 & 4.90220400 & -1.47716900 \\
\hline C & 2.08769700 & 4.35038200 & -2.22781400 \\
\hline $\mathrm{H}$ & -1.16546200 & 3.35246500 & -0.37469500 \\
\hline $\mathrm{H}$ & 2.81148600 & 2.38546400 & -1.69447300 \\
\hline $\mathrm{H}$ & -0.97260300 & 5.59550400 & -1.43001400 \\
\hline $\mathrm{H}$ & 2.99436700 & 4.61737600 & -2.76450200 \\
\hline C & -0.01224300 & -0.59226800 & -0.85672700 \\
\hline $\mathrm{H}$ & -0.87456400 & -0.21118100 & -1.41554500 \\
\hline $\mathrm{H}$ & 1.11402000 & 6.22800300 & -2.62143400 \\
\hline $\mathrm{Rh}$ & -3.32466400 & -0.56540500 & 0.16649500 \\
\hline $\mathrm{O}$ & -2.70860300 & -1.55166200 & -1.55360700 \\
\hline C & -3.52091600 & -1.63145500 & -2.52362700 \\
\hline $\mathrm{O}$ & -4.67647800 & -1.16096300 & -2.62651000 \\
\hline $\mathrm{Rh}$ & -5.46644800 & -0.11967200 & -1.02225600 \\
\hline $\mathrm{O}$ & -4.55734800 & 1.62800500 & -1.71437200 \\
\hline C & -3.39556000 & 1.90601100 & -1.35767900 \\
\hline $\mathrm{O}$ & -2.61694300 & 1.26083100 & -0.58360300 \\
\hline $\mathrm{H}$ & -2.96994900 & 2.84364000 & -1.76477900 \\
\hline $\mathrm{O}$ & -6.14923700 & -1.89660600 & -0.22650000 \\
\hline C & -5.35744000 & -2.57860900 & 0.46953700 \\
\hline $\mathrm{O}$ & -4.16030600 & -2.32965600 & 0.79356100 \\
\hline $\mathrm{H}$ & -5.75051700 & -3.53321800 & 0.85930100 \\
\hline $\mathrm{O}$ & -6.08469600 & 0.86132000 & 0.69646700 \\
\hline C & -5.31101000 & 0.89635600 & 1.67954500 \\
\hline $\mathrm{O}$ & -4.13566800 & 0.43171300 & 1.78739900 \\
\hline $\mathrm{H}$ & -5.69179300 & 1.40383800 & 2.58376100 \\
\hline $\mathrm{H}$ & -3.14488000 & -2.19329200 & -3.39712700 \\
\hline C & -0.24033500 & -0.36087200 & 0.64459700 \\
\hline $\mathrm{H}$ & 0.63182400 & -0.79132500 & 1.18246300 \\
\hline C & 0.41985200 & -1.92162400 & -1.38630100 \\
\hline $\mathrm{H}$ & 1.18959500 & -2.39075800 & -0.77006700 \\
\hline H & -0.46103100 & -2.56596800 & -1.40884900 \\
\hline $\mathrm{H}$ & 0.79536700 & -1.79871100 & -2.40780400 \\
\hline C & -0.25050500 & 2.06203500 & 1.79375900 \\
\hline C & 0.82692500 & 2.84775600 & 2.23751700 \\
\hline C & -1.50877200 & 2.19828600 & 2.39509800 \\
\hline C & 0.64972600 & 3.73964700 & 3.28275500 \\
\hline $\mathrm{H}$ & 1.79952400 & 2.72684200 & 1.76555400 \\
\hline C & -1.67485900 & 3.11237900 & 3.42777400 \\
\hline H & -2.36469400 & 1.63259000 & 2.03851200 \\
\hline C & -0.60261600 & 3.87531400 & 3.87795300 \\
\hline $\mathrm{H}$ & 1.49009800 & 4.33312400 & 3.63380100 \\
\hline
\end{tabular}




$\begin{array}{lrrr}\mathrm{H} & -2.65568400 & 3.22168700 & 3.88312400 \\ \mathrm{H} & -0.74174700 & 4.57998000 & 4.69435000 \\ \mathrm{O} & 1.07971800 & 0.39586700 & -1.12215400 \\ \mathrm{C} & 0.00729800 & 1.11559100 & 0.71220000 \\ \mathrm{H} & -1.73463100 & -0.23952300 & 2.20235300 \\ \mathrm{Rh} & 3.35729600 & -0.36219500 & -0.46154000 \\ \mathrm{O} & 3.50459100 & -1.93831700 & -1.77045100 \\ \mathrm{C} & 4.56602500 & -2.62092700 & -1.77318700 \\ \mathrm{O} & 5.59120800 & -2.46555000 & -1.05646400 \\ \mathrm{Rh} & 5.58372200 & -0.91569400 & 0.27249600 \\ \mathrm{O} & 4.76674800 & -2.13719800 & 1.69676800 \\ \mathrm{C} & 3.51256900 & -2.21127100 & 1.75426400 \\ \mathrm{O} & 2.66905800 & -1.61532100 & 1.02440600 \\ \mathrm{H} & 3.09394800 & -2.87132900 & 2.52935000 \\ \mathrm{O} & 6.31298200 & 0.31871800 & -1.19197400 \\ \mathrm{C} & 5.48458400 & 0.89886300 & -1.93822300 \\ \mathrm{O} & 4.22193700 & 0.83568100 & -1.89436800 \\ \mathrm{H} & 5.90509400 & 1.54242800 & -2.72667300 \\ \mathrm{O} & 5.46074800 & 0.67214500 & 1.57761000 \\ \mathrm{C} & 4.40263300 & 1.34674900 & 1.59825500 \\ \mathrm{O} & 3.36497800 & 1.19130700 & 0.88853400 \\ \mathrm{H} & 4.36599000 & 2.17862700 & 2.32106500 \\ \mathrm{H} & 4.59832800 & -3.45538400 & -2.49059200\end{array}$

TS(c)-IV-2Rh $\mathrm{L}_{\mathbf{4}}$ (-2970.497803 a.u.)

$\begin{array}{lrrr}\mathrm{C} & 1.05235100 & -0.83989200 & 0.12345800 \\ \mathrm{C} & 0.86759600 & -1.28847100 & 1.52078200 \\ \mathrm{O} & 0.65531100 & -0.26468300 & 2.35433800 \\ \mathrm{C} & 0.58106300 & -0.58615600 & 3.73454700 \\ \mathrm{H} & 1.49564500 & -1.09454700 & 4.05618000 \\ \mathrm{H} & -0.27783600 & -1.23440300 & 3.94046000 \\ \mathrm{H} & 0.46974200 & 0.36846600 & 4.25393000 \\ \mathrm{O} & 0.89869000 & -2.44794000 & 1.87026200 \\ \mathrm{~N} & -1.35777500 & 1.90710100 & -0.59992900 \\ \mathrm{C} & -1.73342800 & 3.21476000 & -0.99537800 \\ \mathrm{C} & -2.50916800 & 5.76143000 & -1.75602700 \\ \mathrm{C} & -0.79032600 & 4.24182600 & -1.01219900 \\ \mathrm{C} & -3.05804100 & 3.44187100 & -1.36758500 \\ \mathrm{C} & -1.19124300 & 5.52010800 & -1.38009400 \\ \mathrm{C} & -3.43396100 & 4.71994200 & -1.75876700 \\ \mathrm{H} & 0.24270700 & 4.03676900 & -0.73709800 \\ \mathrm{H} & -3.76964600 & 2.61832000 & -1.36085100 \\ \mathrm{H} & -0.46277200 & 6.32696500 & -1.38911600 \\ \mathrm{H} & -4.46210400 & 4.90499300 & -2.05975200 \\ & & & \\ & & 5135 & \end{array}$




\begin{tabular}{|c|c|c|c|}
\hline C & -0.49181200 & 0.14048300 & -1.74185300 \\
\hline $\mathrm{H}$ & -0.81689600 & -0.89364800 & -1.90321500 \\
\hline $\mathrm{H}$ & -2.81477700 & 6.76141700 & -2.05340400 \\
\hline $\mathrm{Rh}$ & 3.30971400 & -0.55094800 & -0.16350800 \\
\hline $\mathrm{O}$ & 3.30527300 & 1.46833800 & -0.72179500 \\
\hline C & 4.41074800 & 1.99166100 & -1.06540200 \\
\hline $\mathrm{O}$ & 5.54546000 & 1.46757900 & -1.09001800 \\
\hline $\mathrm{Rh}$ & 5.73940900 & -0.51162100 & -0.51277200 \\
\hline $\mathrm{O}$ & 5.35117400 & -1.10952100 & -2.45597000 \\
\hline C & 4.16129800 & -1.24509900 & -2.82423200 \\
\hline $\mathrm{O}$ & 3.09941400 & -1.07041600 & -2.15206300 \\
\hline $\mathrm{H}$ & 4.00562800 & -1.55540900 & -3.87109000 \\
\hline $\mathrm{O}$ & 5.91227200 & 0.07121700 & 1.46130100 \\
\hline C & 4.86900700 & 0.15250200 & 2.15348700 \\
\hline $\mathrm{O}$ & 3.66692300 & -0.03915400 & 1.80403600 \\
\hline $\mathrm{H}$ & 5.00720700 & 0.43321300 & 3.21169000 \\
\hline $\mathrm{O}$ & 5.76321100 & -2.48026600 & 0.07972300 \\
\hline C & 4.66291700 & -3.03351700 & 0.33088700 \\
\hline $\mathrm{O}$ & 3.50264300 & -2.53227200 & 0.30362500 \\
\hline $\mathrm{H}$ & 4.70301200 & -4.09807300 & 0.61351100 \\
\hline $\mathrm{H}$ & 4.34869700 & 3.04736100 & -1.37920500 \\
\hline C & 0.21719000 & 0.31910100 & -0.41255100 \\
\hline $\mathrm{H}$ & 1.47640000 & 0.37609700 & -0.41986500 \\
\hline C & 0.25426200 & 0.70565600 & -2.92273000 \\
\hline $\mathrm{H}$ & 0.48849000 & 1.76441100 & -2.75329600 \\
\hline $\mathrm{H}$ & 1.20132300 & 0.16243700 & -3.03597800 \\
\hline $\mathrm{H}$ & -0.33072500 & 0.60839800 & -3.84197300 \\
\hline C & 0.04013100 & 2.25273500 & 1.35100100 \\
\hline C & 1.37161900 & 2.45885200 & 1.72441100 \\
\hline C & -0.99467400 & 2.76446100 & 2.14335600 \\
\hline C & 1.66162800 & 3.15625100 & 2.88795100 \\
\hline $\mathrm{H}$ & 2.17621100 & 2.07098900 & 1.10502200 \\
\hline C & -0.69451700 & 3.45971300 & 3.30736800 \\
\hline $\mathrm{H}$ & -2.02609600 & 2.58206200 & 1.84829500 \\
\hline C & 0.63211000 & 3.65602000 & 3.68268400 \\
\hline $\mathrm{H}$ & 2.69951700 & 3.30758500 & 3.17583600 \\
\hline H & -1.50014900 & 3.84717400 & 3.92633800 \\
\hline $\mathrm{H}$ & 0.86420500 & 4.20055900 & 4.59507400 \\
\hline $\mathrm{O}$ & -1.72820600 & 0.89240900 & -1.51398900 \\
\hline C & -0.30298600 & 1.48251100 & 0.15575200 \\
\hline $\mathrm{H}$ & 0.99716900 & -1.66456100 & -0.58820800 \\
\hline $\mathrm{Rh}$ & -3.32853300 & -0.42442700 & -0.51575900 \\
\hline $\mathrm{O}$ & -3.65056300 & 0.87629200 & 1.05210500 \\
\hline C & -4.49932700 & 0.54140100 & 1.92805700 \\
\hline
\end{tabular}




$\begin{array}{lrrr}\text { O } & -5.20449300 & -0.49785800 & 1.97048100 \\ \text { Rh } & -4.98995100 & -1.87017800 & 0.45478500 \\ \text { O } & -3.48576400 & -2.79162100 & 1.50631100 \\ \text { C } & -2.30881600 & -2.37796000 & 1.34146100 \\ \text { O } & -1.92355300 & -1.42930600 & 0.59489500 \\ \text { H } & -1.50758700 & -2.88751500 & 1.90274800 \\ \text { O } & -6.39575000 & -0.86255600 & -0.66138100 \\ \text { C } & -6.00845600 & 0.07504300 & -1.40411100 \\ \text { O } & -4.83062800 & 0.50536600 & -1.57079200 \\ \text { H } & -6.79058300 & 0.59009900 & -1.98474100 \\ \text { O } & -4.66972100 & -3.15575400 & -1.11658800 \\ \mathrm{C} & -3.80866800 & -2.84275000 & -1.97689700 \\ \text { O } & -3.09169300 & -1.80059500 & -2.01929400 \\ \text { H } & -3.65539300 & -3.56003200 & -2.79848300 \\ \text { H } & -4.63465300 & 1.25500500 & 2.75741200\end{array}$

s-trans r-4 (-4598.121151 a.u)

$\begin{array}{lrrr}\text { C } & -1.60660000 & 2.05048800 & -0.18961600 \\ \text { C } & -2.07613500 & -1.63024100 & -0.43536700 \\ \text { C } & 1.44360700 & -2.12573600 & -1.01157600 \\ \text { C } & 2.01508400 & 1.59462200 & -0.77545000 \\ \text { O } & -1.44397500 & -1.37134500 & 0.63683300 \\ \text { O } & -1.81521200 & -1.18265400 & -1.58534800 \\ \text { O } & 1.27461100 & -1.80253700 & 0.21113100 \\ \text { O } & 1.06739400 & -1.47847400 & -2.02062900 \\ \text { O } & 1.78534200 & 1.06339600 & 0.35575700 \\ \text { O } & 1.35043600 & 1.43241000 & -1.83324600 \\ \text { O } & -1.01298400 & 1.56571700 & 0.82427000 \\ \text { O } & -1.49710800 & 1.65840100 & -1.38127900 \\ \text { Rh } & 0.16603900 & -0.12479900 & 0.61337100 \\ \text { Rh } & -0.22493500 & 0.10943800 & -1.80322900 \\ \text { C } & 2.06864000 & -3.47010200 & -1.28369800 \\ \text { C } & 3.15678700 & -3.49477200 & -2.31040300 \\ \text { C } & 3.51263900 & -3.77388100 & -0.87934600 \\ \text { C } & 1.03870300 & -4.56253100 & -1.20249800 \\ \text { H } & 3.18958400 & -4.31345600 & -3.02486100 \\ \text { H } & 3.43724300 & -2.52651900 & -2.72242600 \\ \text { C } & 4.35293200 & -2.77659800 & -0.13540900 \\ \text { C } & 3.83723300 & -5.19376800 & -0.50722400 \\ \text { C } & 0.24001200 & -4.67821700 & -0.05819400 \\ \text { C } & 0.88029100 & -5.50278400 & -2.22057600 \\ \text { C } & 4.02241400 & -2.36043600 & 1.15545900 \\ \text { C } & 5.55463400 & -2.33929600 & -0.69587500 \\ \text { C } & 3.37542400 & -5.73661700 & 0.69375500\end{array}$




\begin{tabular}{|c|c|c|c|}
\hline C & 4.64404400 & -5.97813900 & -1.32987800 \\
\hline $\mathrm{H}$ & 0.32559600 & -3.92642800 & 0.72649900 \\
\hline C & -0.64669900 & -5.73926600 & 0.08208200 \\
\hline C & -0.01840300 & -6.55623800 & -2.08644200 \\
\hline $\mathrm{H}$ & 1.46740700 & -5.41722800 & -3.13159800 \\
\hline $\mathrm{H}$ & 3.09144700 & -2.68669500 & 1.61066300 \\
\hline C & 4.86450400 & -1.51132600 & 1.86225100 \\
\hline C & 6.39948100 & -1.48523800 & 0.00684900 \\
\hline $\mathrm{H}$ & 5.82797800 & -2.68352200 & -1.69426400 \\
\hline $\mathrm{H}$ & 2.74678000 & -5.12674800 & 1.34210600 \\
\hline C & 3.69495000 & -7.03883700 & 1.05368100 \\
\hline C & 4.97259500 & -7.28166000 & -0.97018400 \\
\hline $\mathrm{H}$ & 5.02102100 & -5.55857400 & -2.26213400 \\
\hline $\mathrm{H}$ & -1.24314700 & -5.82574400 & 0.98891400 \\
\hline C & -0.77568500 & -6.68858500 & -0.92791100 \\
\hline $\mathrm{H}$ & -0.12123000 & -7.27938600 & -2.89232100 \\
\hline $\mathrm{H}$ & 4.57163300 & -1.19782700 & 2.86268700 \\
\hline C & 6.05590000 & -1.06988200 & 1.29044600 \\
\hline $\mathrm{H}$ & 7.32222700 & -1.13475900 & -0.45345100 \\
\hline $\mathrm{H}$ & 3.31724400 & -7.45017900 & 1.98722800 \\
\hline C & 4.49466200 & -7.81707500 & 0.22046100 \\
\hline $\mathrm{H}$ & 5.60300600 & -7.88028700 & -1.62415900 \\
\hline $\mathrm{H}$ & 6.70991200 & -0.39511400 & 1.84141900 \\
\hline $\mathrm{H}$ & 4.74441100 & -8.83790300 & 0.50061200 \\
\hline C & 3.21603100 & 2.48782100 & -0.87219800 \\
\hline C & 2.95399300 & 3.93705400 & -0.56977400 \\
\hline C & 3.79106800 & 3.15292500 & 0.38415300 \\
\hline C & 4.11345900 & 2.06585200 & -1.99161700 \\
\hline $\mathrm{H}$ & 3.43999800 & 4.68641300 & -1.18943500 \\
\hline $\mathrm{H}$ & 1.93534000 & 4.18911700 & -0.27737500 \\
\hline C & 3.26817900 & 2.90217500 & 1.77184100 \\
\hline C & 5.26953600 & 3.42899200 & 0.38886800 \\
\hline C & 4.52677800 & 0.73012400 & -2.03500000 \\
\hline C & 4.54579500 & 2.93652500 & -2.98763400 \\
\hline C & 3.66717100 & 1.76684100 & 2.48328300 \\
\hline C & 2.47786800 & 3.85366600 & 2.41443400 \\
\hline C & 6.19811900 & 2.38817600 & 0.33184600 \\
\hline C & 5.73272600 & 4.73731900 & 0.52232400 \\
\hline $\mathrm{H}$ & 4.20000200 & 0.03664600 & -1.25287200 \\
\hline C & 5.36616300 & 0.28254600 & -3.04480400 \\
\hline C & 5.39133000 & 2.48943900 & -3.99930600 \\
\hline $\mathrm{H}$ & 4.21358300 & 3.97344700 & -2.98129600 \\
\hline $\mathrm{H}$ & 4.25281500 & 1.00319400 & 1.97300800 \\
\hline C & 3.30700900 & 1.59750800 & 3.81071200 \\
\hline
\end{tabular}




\begin{tabular}{|c|c|c|c|}
\hline C & 2.11587800 & 3.68924400 & 3.75123300 \\
\hline $\mathrm{H}$ & 2.16036800 & 4.74432300 & 1.87153600 \\
\hline $\mathrm{H}$ & 5.84758800 & 1.36339200 & 0.22023400 \\
\hline C & 7.56064800 & 2.65102000 & 0.39418300 \\
\hline C & 7.09675600 & 5.00415700 & 0.58677200 \\
\hline $\mathrm{H}$ & 5.00941700 & 5.55093100 & 0.58317700 \\
\hline $\mathrm{H}$ & 5.68291000 & -0.75925100 & -3.05548000 \\
\hline C & 5.80528300 & 1.16309400 & -4.03086900 \\
\hline $\mathrm{H}$ & 5.72338400 & 3.18290800 & -4.76882800 \\
\hline $\mathrm{H}$ & 3.61058500 & 0.69749600 & 4.34285800 \\
\hline C & 2.54226700 & 2.56952500 & 4.45510200 \\
\hline $\mathrm{H}$ & 1.51380100 & 4.45042500 & 4.24527200 \\
\hline $\mathrm{H}$ & 8.26965900 & 1.82631800 & 0.33731600 \\
\hline C & 8.01499300 & 3.96105100 & 0.52032100 \\
\hline $\mathrm{H}$ & 7.44349800 & 6.03028100 & 0.68952100 \\
\hline $\mathrm{H}$ & 2.28711700 & 2.45363100 & 5.50737500 \\
\hline $\mathrm{H}$ & 9.08181700 & 4.16861400 & 0.56565700 \\
\hline C & -2.59020900 & 3.16304600 & 0.07139200 \\
\hline C & -2.19606100 & 4.16873200 & 1.11095200 \\
\hline C & -2.21809900 & 4.59693600 & -0.32640300 \\
\hline C & -4.02105500 & 2.71240000 & -0.08846000 \\
\hline $\mathrm{H}$ & -2.94960600 & 4.56035900 & 1.78975600 \\
\hline $\mathrm{H}$ & -1.21615600 & 4.01530900 & 1.56321000 \\
\hline C & -0.92317900 & 4.96822500 & -0.99676500 \\
\hline C & -3.35107200 & 5.47128900 & -0.78753500 \\
\hline C & -4.39827200 & 1.90253200 & -1.17045300 \\
\hline C & -5.01098300 & 3.09106000 & 0.82131900 \\
\hline C & -0.48160800 & 4.38543600 & -2.18669500 \\
\hline C & -0.22152400 & 6.06556100 & -0.48321200 \\
\hline C & -3.90298000 & 5.27602300 & -2.05557200 \\
\hline C & -3.84317500 & 6.50182600 & 0.01047600 \\
\hline $\mathrm{H}$ & -3.64889600 & 1.58859600 & -1.89326000 \\
\hline C & -5.71696700 & 1.48891700 & -1.32396000 \\
\hline C & -6.32872600 & 2.67107500 & 0.67115600 \\
\hline $\mathrm{H}$ & -4.75944100 & 3.72255600 & 1.67039300 \\
\hline $\mathrm{H}$ & -1.00008200 & 3.51790200 & -2.58624200 \\
\hline C & 0.62739600 & 4.90066100 & -2.85384600 \\
\hline C & 0.87132200 & 6.59310700 & -1.15951800 \\
\hline $\mathrm{H}$ & -0.56575700 & 6.52410900 & 0.44428000 \\
\hline $\mathrm{H}$ & -3.51821900 & 4.47143200 & -2.68231000 \\
\hline C & -4.93900400 & 6.08265000 & -2.50660800 \\
\hline C & -4.87769000 & 7.31632900 & -0.44131700 \\
\hline H & -3.41078200 & 6.66888300 & 0.99671600 \\
\hline $\mathrm{H}$ & -5.98472000 & 0.86737800 & -2.17696100 \\
\hline
\end{tabular}




\begin{tabular}{|c|c|c|c|}
\hline C & -6.68944900 & 1.86660700 & -0.40307100 \\
\hline $\mathrm{H}$ & -7.07398700 & 2.97726300 & 1.40154900 \\
\hline $\mathrm{H}$ & 0.97066900 & 4.41925400 & -3.76715500 \\
\hline C & 1.29099400 & 6.01615000 & -2.35639300 \\
\hline $\mathrm{H}$ & 1.39496700 & 7.45640000 & -0.75405300 \\
\hline $\mathrm{H}$ & -5.36744300 & 5.91132600 & -3.49152700 \\
\hline C & -5.43065100 & 7.10566900 & -1.69896900 \\
\hline $\mathrm{H}$ & -5.25247400 & 8.11648700 & 0.19338900 \\
\hline $\mathrm{H}$ & 2.14807300 & 6.42468800 & -2.88916900 \\
\hline $\mathrm{H}$ & -6.24323700 & 7.73686600 & -2.05146600 \\
\hline C & -3.22474600 & -2.57579400 & -0.29764500 \\
\hline C & -3.11530300 & -3.60770300 & 0.79613300 \\
\hline C & -4.11045200 & -2.51592400 & 0.98177300 \\
\hline C & -3.84787500 & -2.96968200 & -1.59795200 \\
\hline $\mathrm{H}$ & -3.44280800 & -4.61358200 & 0.54598400 \\
\hline $\mathrm{H}$ & -2.22036800 & -3.54513800 & 1.41354200 \\
\hline C & -3.83177700 & -1.52501300 & 2.07692900 \\
\hline C & -5.57740400 & -2.77967500 & 0.75913800 \\
\hline C & -4.79121500 & -2.14362300 & -2.20892400 \\
\hline C & -3.45209800 & -4.14261400 & -2.23634000 \\
\hline C & -3.82973700 & -0.14761500 & 1.85836300 \\
\hline C & -3.64332600 & -1.99686500 & 3.37639400 \\
\hline C & -6.47938600 & -1.70932900 & 0.76018200 \\
\hline C & -6.07453800 & -4.05508800 & 0.48564000 \\
\hline $\mathrm{H}$ & -5.08253700 & -1.21874800 & -1.71022600 \\
\hline C & -5.35641000 & -2.50110500 & -3.42534200 \\
\hline C & -4.01076200 & -4.49905100 & -3.46017400 \\
\hline $\mathrm{H}$ & -2.68998800 & -4.77320300 & -1.77540700 \\
\hline $\mathrm{H}$ & -3.95889900 & 0.23551600 & 0.84466000 \\
\hline C & -3.65666500 & 0.73819200 & 2.91458900 \\
\hline C & -3.47409200 & -1.11440300 & 4.43847200 \\
\hline $\mathrm{H}$ & -3.63592500 & -3.07241200 & 3.55197700 \\
\hline $\mathrm{H}$ & -6.12581400 & -0.70257400 & 0.97746900 \\
\hline C & -7.82238100 & -1.90543900 & 0.46621300 \\
\hline C & -7.41947700 & -4.25423300 & 0.19404100 \\
\hline $\mathrm{H}$ & -5.40627500 & -4.91285700 & 0.48065100 \\
\hline $\mathrm{H}$ & -6.10127500 & -1.85568200 & -3.88707800 \\
\hline C & -4.96938600 & -3.68387600 & -4.05241900 \\
\hline $\mathrm{H}$ & -3.69275100 & -5.41625300 & -3.95160500 \\
\hline $\mathrm{H}$ & -3.64739300 & 1.80868200 & 2.71681600 \\
\hline C & -3.49188500 & 0.25832900 & 4.20965600 \\
\hline $\mathrm{H}$ & -3.34000200 & -1.50016800 & 5.44785500 \\
\hline $\mathrm{H}$ & -8.49947400 & -1.05322000 & 0.46560300 \\
\hline C & -8.30036300 & -3.17949500 & 0.17649200 \\
\hline
\end{tabular}




$\begin{array}{lccc}H & -7.77716200 & -5.25828400 & -0.02319100 \\ H & -3.38244100 & 0.95564600 & 5.03861800 \\ H & -9.35135300 & -3.33375800 & -0.05693400 \\ H & -7.71966700 & 1.54010200 & -0.52661800 \\ H & -5.41273000 & -3.96582000 & -5.00503000 \\ H & -1.46729400 & -7.52045500 & -0.81516500 \\ H & 6.46570000 & 0.81436300 & -4.82155100 \\ \mathrm{C} & 0.34238500 & -0.30720400 & 2.55659600 \\ \mathrm{C} & 0.88455700 & -1.56801800 & 3.09666700 \\ \mathrm{O} & 0.01336000 & -2.57883700 & 3.01203000 \\ \mathrm{C} & 0.50112700 & -3.83746400 & 3.46935600 \\ \mathrm{H} & 0.82865700 & -3.77623000 & 4.51157700 \\ \mathrm{H} & 1.34572600 & -4.16999300 & 2.85403700 \\ \mathrm{H} & -0.33368800 & -4.53390000 & 3.37127400 \\ \mathrm{O} & 2.00811500 & -1.66749600 & 3.54584000 \\ \mathrm{C} & -0.09811800 & 0.53740300 & 4.79498400 \\ \mathrm{H} & 0.27837100 & -0.39866700 & 5.21535200 \\ \mathrm{C} & -0.08942900 & 0.70123000 & 3.44654300 \\ \mathrm{H} & -0.45257400 & 1.63499900 & 3.01300700 \\ \mathrm{C} & -0.58141900 & 1.55267400 & 5.75294900 \\ \mathrm{H} & 0.21654200 & 1.84014000 & 6.45070600 \\ \mathrm{H} & -0.94663700 & 2.44976300 & 5.24183500 \\ \mathrm{H} & -1.38877400 & 1.13726200 & 6.37161700\end{array}$

r-TS(tx)-I (-5229.5783 a.u.)

$\begin{array}{lrrr}\text { C } & -0.80182700 & 2.59733000 & -0.57677200 \\ \text { C } & -2.17232000 & -1.01114500 & -1.04274800 \\ \text { C } & 1.15615500 & -2.23160300 & -1.24498700 \\ \text { C } & 2.57542100 & 1.19785000 & -0.74889400 \\ \text { O } & -1.67862200 & -0.94016900 & 0.12987700 \\ \text { O } & -1.74623000 & -0.41199400 & -2.06768500 \\ \text { O } & 1.06638800 & -1.83953500 & -0.03524600 \\ \text { O } & 0.89833100 & -1.55635000 & -2.27326800 \\ \text { O } & 2.01411800 & 0.92304400 & 0.35879400 \\ \text { O } & 2.07854600 & 1.06136200 & -1.90015000 \\ \text { O } & -0.65874300 & 1.91877500 & 0.49037700 \\ \text { O } & -0.56258800 & 2.21615800 & -1.75118100 \\ \text { Rh } & 0.18822800 & 0.00583400 & 0.34698600 \\ \text { Rh } & 0.16747500 & 0.34711000 & -2.09575800 \\ \text { C } & 1.49967800 & -3.67985700 & -1.51587500 \\ \text { C } & 2.51742600 & -3.89358400 & -2.59569500 \\ \text { C } & 2.87138300 & -4.29070400 & -1.19578900 \\ \text { C } & 0.30250500 & -4.57689800 & -1.36694900 \\ \text { H } & 2.35540100 & -4.66635200 & -3.34279300 \\ & & & \\ & & 5141 & \end{array}$




\begin{tabular}{ccc}
2.96973900 & -2.98239800 & -2.98504500 \\
3.95198000 & -3.55786900 & -0.45892400 \\
2.89954900 & -5.76515900 & -0.88834900 \\
-0.54353500 & -4.42450600 & -0.26194800 \\
0.06479800 & -5.64245500 & -2.23862500 \\
3.76559900 & -3.10105200 & 0.84612700 \\
5.22703400 & -3.48163500 & -1.02435000 \\
2.34234200 & -6.26075600 & 0.29179900 \\
3.50759500 & -6.66044200 & -1.76707300 \\
-0.41159500 & -3.57296100 & 0.40438800 \\
-1.54116500 & -5.35788800 & 0.00147700 \\
-0.94139600 & -6.56696800 & -1.98210900 \\
0.68579400 & -5.77293500 & -3.12097400 \\
2.77338200 & -3.11599500 & 1.28775000 \\
4.83775400 & -2.60930800 & 1.58143200 \\
6.29682500 & -2.96631200 & -0.30000500 \\
5.37739100 & -3.84795600 & -2.04081400 \\
1.85573700 & -5.56966200 & 0.97780500 \\
2.36235500 & -7.62023800 & 0.57173400 \\
3.53938000 & -8.02319900 & -1.48642800 \\
3.95457000 & -6.28385400 & -2.68676400 \\
-2.16691600 & -5.22871200 & 0.88583400 \\
-1.73886300 & -6.44172800 & -0.84833300 \\
-1.09519800 & -7.39548000 & -2.67001700 \\
4.66821900 & -2.27445900 & 2.60287500 \\
6.10744900 & -2.54486600 & 1.01339800 \\
7.28050000 & -2.89641100 & -0.76115900 \\
1.90490900 & -7.98989900 & 1.48688700 \\
2.95977800 & -8.50797300 & -0.31953500 \\
4.01442300 & -8.70787700 & -2.18582400 \\
6.94370400 & -2.15434100 & 1.59088800 \\
2.97361700 & -9.57388100 & -0.10343000 \\
3.95906900 & 1.77612300 & -0.72165800 \\
4.01795600 & 3.21595600 & -0.26704800 \\
4.67938300 & 2.18343800 & 0.57361600 \\
4.80096000 & 1.27805900 & -1.85620000 \\
4.62232000 & 3.91118900 & -0.84200400 \\
3.07835400 & 3.63513100 & 0.09489400 \\
4.19933200 & 1.93535700 & 1.97298000 \\
6.18315100 & 2.10882700 & 0.50614800 \\
4.90729300 & -0.10549100 & -2.03199300 \\
5.53093700 & 2.11511200 & -2.69476900 \\
4.05370100 & 0.64872900 & 2.48782700 \\
\hline & 3.02671100 & 2.83506800 \\
\hline
\end{tabular}




\begin{tabular}{|c|c|c|c|}
\hline C & 6.81526700 & 0.86487300 & 0.46811000 \\
\hline C & 6.96540400 & 3.26117900 & 0.49716600 \\
\hline $\mathrm{H}$ & 4.34864600 & -0.77721800 & -1.37286100 \\
\hline C & 5.73279400 & -0.63574600 & -3.01213900 \\
\hline C & 6.36681500 & 1.58557000 & -3.67418300 \\
\hline $\mathrm{H}$ & 5.45682800 & 3.19514500 & -2.57980200 \\
\hline $\mathrm{H}$ & 4.08976500 & -0.20363700 & 1.81053400 \\
\hline C & 3.84388600 & 0.45319100 & 3.84696700 \\
\hline C & 3.86230100 & 2.83614500 & 4.19627100 \\
\hline $\mathrm{H}$ & 4.18909600 & 4.03399900 & 2.43323700 \\
\hline $\mathrm{H}$ & 6.20918100 & -0.04009400 & 0.47713600 \\
\hline C & 8.19703300 & 0.77486100 & 0.38046600 \\
\hline C & 8.35333500 & 3.17501700 & 0.42403400 \\
\hline $\mathrm{H}$ & 6.48068100 & 4.23641000 & 0.54122300 \\
\hline $\mathrm{H}$ & 5.80443400 & -1.71660200 & -3.12242100 \\
\hline C & 6.47265800 & 0.20953200 & -3.83584500 \\
\hline $\mathrm{H}$ & 6.93709600 & 2.25592200 & -4.31368600 \\
\hline $\mathrm{H}$ & 3.71685400 & -0.55746200 & 4.22673200 \\
\hline C & 3.76930400 & 1.54495100 & 4.70815200 \\
\hline $\mathrm{H}$ & 3.79011600 & 3.69617100 & 4.86003500 \\
\hline $\mathrm{H}$ & 8.67094500 & -0.20406700 & 0.32487500 \\
\hline C & 8.97248300 & 1.93215200 & 0.35569900 \\
\hline $\mathrm{H}$ & 8.95146500 & 4.08389900 & 0.41371700 \\
\hline $\mathrm{H}$ & 3.63457400 & 1.38783500 & 5.77779800 \\
\hline $\mathrm{H}$ & 10.05578000 & 1.86416200 & 0.28372600 \\
\hline C & -1.39389500 & 3.98028300 & -0.46484900 \\
\hline C & -0.80544300 & 4.90170400 & 0.56152200 \\
\hline C & -0.54579600 & 5.19524700 & -0.88586900 \\
\hline C & -2.85893500 & 4.00384300 & -0.81886300 \\
\hline $\mathrm{H}$ & -1.45383600 & 5.56391800 & 1.13076100 \\
\hline $\mathrm{H}$ & 0.02250100 & 4.48543900 & 1.13676000 \\
\hline C & 0.86482700 & 5.14294500 & -1.41218000 \\
\hline C & -1.30520300 & 6.31977300 & -1.53574300 \\
\hline C & -3.37296800 & 3.11752400 & -1.77546600 \\
\hline C & -3.73173700 & 4.95869300 & -0.28336600 \\
\hline C & 1.25279900 & 4.39398100 & -2.52549900 \\
\hline C & 1.77740200 & 6.05286100 & -0.86423600 \\
\hline C & -1.79256900 & 6.15526800 & -2.83445900 \\
\hline C & -1.52260600 & 7.53018300 & -0.88140100 \\
\hline $\mathrm{H}$ & -2.72657700 & 2.36385700 & -2.22129600 \\
\hline C & -4.70309600 & 3.18995800 & -2.17768300 \\
\hline C & -5.05697400 & 5.03930600 & -0.69515500 \\
\hline $\mathrm{H}$ & -3.36885700 & 5.68748300 & 0.43742800 \\
\hline $\mathrm{H}$ & 0.56484400 & 3.67323900 & -2.95632000 \\
\hline
\end{tabular}




\begin{tabular}{|c|c|c|c|}
\hline C & 2.52177100 & 4.55563900 & -3.07564300 \\
\hline C & 3.03222300 & 6.23596100 & -1.43067200 \\
\hline $\mathrm{H}$ & 1.47682000 & 6.64569900 & -0.00002100 \\
\hline $\mathrm{H}$ & -1.62173100 & 5.20816700 & -3.34660600 \\
\hline C & -2.51596700 & 7.16700100 & -3.45023700 \\
\hline C & -2.24188800 & 8.54983900 & -1.49909600 \\
\hline $\mathrm{H}$ & -1.13427400 & 7.67190200 & 0.12732700 \\
\hline $\mathrm{H}$ & -5.07082300 & 2.47936100 & -2.91655800 \\
\hline C & -5.55245800 & 4.15288900 & -1.64599300 \\
\hline $\mathrm{H}$ & -5.70245300 & 5.80359900 & -0.26855400 \\
\hline $\mathrm{H}$ & 2.81516900 & 3.94297000 & -3.92548500 \\
\hline C & 3.40494800 & 5.48985700 & -2.54639000 \\
\hline $\mathrm{H}$ & 3.72014700 & 6.96139600 & -1.00105700 \\
\hline $\mathrm{H}$ & -2.90607400 & 7.01814400 & -4.45451900 \\
\hline C & -2.74732600 & 8.36712000 & -2.78101400 \\
\hline $\mathrm{H}$ & -2.41000700 & 9.48789100 & -0.97423100 \\
\hline $\mathrm{H}$ & 4.38783000 & 5.62900600 & -2.99421500 \\
\hline $\mathrm{H}$ & -3.31802300 & 9.15879600 & -3.26117900 \\
\hline C & -3.33649600 & -1.94256600 & -1.28906200 \\
\hline C & -3.70870800 & -2.88971600 & -0.18659500 \\
\hline C & -4.68230900 & -1.81205900 & -0.53304500 \\
\hline C & -3.41819400 & -2.41675200 & -2.70946800 \\
\hline $\mathrm{H}$ & -3.91752500 & -3.92098400 & -0.45913700 \\
\hline $\mathrm{H}$ & -3.13748700 & -2.76556800 & 0.73164500 \\
\hline C & -4.93760700 & -0.71259300 & 0.44903700 \\
\hline C & -5.91057700 & -2.14015100 & -1.34316000 \\
\hline C & -3.92643400 & -1.57763900 & -3.70349200 \\
\hline C & -3.00205400 & -3.69842000 & -3.05378500 \\
\hline C & -4.58944000 & 0.61181000 & 0.19054600 \\
\hline C & -5.69526100 & -0.99922900 & 1.58848400 \\
\hline C & -6.70105800 & -1.09423200 & -1.83306500 \\
\hline C & -6.26713400 & -3.44655200 & -1.67970900 \\
\hline $\mathrm{H}$ & -4.24129200 & -0.56916900 & -3.43387400 \\
\hline C & -4.04138200 & -2.02453200 & -5.01149300 \\
\hline C & -3.10430300 & -4.14659800 & -4.36823400 \\
\hline $\mathrm{H}$ & -2.60312700 & -4.35791200 & -2.28548000 \\
\hline $\mathrm{H}$ & -4.02061600 & 0.85147800 & -0.70828000 \\
\hline C & -4.99961800 & 1.63168800 & 1.04490400 \\
\hline C & -6.07540700 & 0.01237700 & 2.46543400 \\
\hline $\mathrm{H}$ & -5.99657300 & -2.03210400 & 1.77232400 \\
\hline $\mathrm{H}$ & -6.44892600 & -0.06656000 & -1.57290100 \\
\hline C & -7.78930900 & -1.34459000 & -2.65808100 \\
\hline C & -7.35666600 & -3.70037800 & -2.50577300 \\
\hline $\mathrm{H}$ & -5.67995000 & -4.28535100 & -1.31346400 \\
\hline
\end{tabular}




\begin{tabular}{|c|c|c|c|}
\hline $\mathrm{H}$ & -4.45227100 & -1.36651700 & -5.77403000 \\
\hline C & -3.63076100 & -3.31425700 & -5.34793600 \\
\hline $\mathrm{H}$ & -2.77124500 & -5.15274600 & -4.61696900 \\
\hline $\mathrm{H}$ & -4.73630400 & 2.66257500 & 0.81429300 \\
\hline C & -5.73327700 & 1.33369900 & 2.18924300 \\
\hline $\mathrm{H}$ & -6.65373800 & -0.22962100 & 3.35457400 \\
\hline $\mathrm{H}$ & -8.38071700 & -0.51280000 & -3.03471000 \\
\hline C & -8.11945500 & -2.65113300 & -3.00441700 \\
\hline $\mathrm{H}$ & -7.60644300 & -4.72759600 & -2.76187700 \\
\hline $\mathrm{H}$ & -6.02545600 & 2.13256200 & 2.86906400 \\
\hline $\mathrm{H}$ & -8.96780300 & -2.84972500 & -3.65525800 \\
\hline $\mathrm{H}$ & -6.58947800 & 4.21278000 & -1.96708100 \\
\hline $\mathrm{H}$ & -3.72213900 & -3.66469400 & -6.37381000 \\
\hline $\mathrm{H}$ & -2.51343300 & -7.17558200 & -0.63714300 \\
\hline $\mathrm{H}$ & 7.12749000 & -0.20415900 & -4.59953200 \\
\hline $\mathrm{C}$ & 0.31436600 & -0.27875000 & 2.35391400 \\
\hline C & 0.79625600 & -1.61315000 & 2.76531100 \\
\hline 0 & -0.10034700 & -2.59065500 & 2.56437200 \\
\hline $\mathrm{C}$ & 0.37146800 & -3.89184200 & 2.90276700 \\
\hline $\mathrm{H}$ & 0.60779100 & -3.95306400 & 3.97025000 \\
\hline $\mathrm{H}$ & 1.27294200 & -4.13321600 & 2.33037800 \\
\hline $\mathrm{H}$ & -0.43600200 & -4.58258100 & 2.65203100 \\
\hline 0 & 1.91521800 & -1.82697600 & 3.19018700 \\
\hline 0 & -1.40864600 & -0.14154800 & 5.39471400 \\
\hline $\mathrm{N}$ & -2.16451900 & -0.56616900 & 4.41330100 \\
\hline C & -2.37311300 & -1.98694300 & 4.33976800 \\
\hline $\mathrm{C}$ & -2.81752900 & -4.72669300 & 4.33065500 \\
\hline $\mathrm{C}$ & -1.74053300 & -2.78688200 & 5.28621700 \\
\hline C & -3.23432500 & -2.53916800 & 3.39699400 \\
\hline $\mathrm{C}$ & -1.97325900 & -4.15724900 & 5.27903700 \\
\hline C & -3.44086400 & -3.91163900 & 3.38825100 \\
\hline $\mathrm{H}$ & -1.08574000 & -2.32304600 & 6.01636000 \\
\hline $\mathrm{H}$ & -3.75746500 & -1.91550800 & 2.67914800 \\
\hline $\mathrm{H}$ & -1.48471400 & -4.78375000 & 6.02145900 \\
\hline $\mathrm{H}$ & -4.11331800 & -4.33856000 & 2.64539300 \\
\hline $\mathrm{C}$ & 0.28639300 & 0.55285400 & 4.67666300 \\
\hline $\mathrm{H}$ & 0.80677700 & -0.34762100 & 5.00693900 \\
\hline $\mathrm{H}$ & -2.99573700 & -5.79916500 & 4.32907900 \\
\hline $\mathrm{C}$ & 0.13805300 & 0.72288900 & 3.27693500 \\
\hline $\mathrm{H}$ & -0.12617600 & 1.71750600 & 2.90871900 \\
\hline $\mathrm{C}$ & 0.36392000 & 1.73914000 & 5.56700100 \\
\hline $\mathrm{H}$ & 1.39135200 & 2.12405900 & 5.53128000 \\
\hline $\mathrm{H}$ & -0.30782900 & 2.53347400 & 5.22295600 \\
\hline $\mathrm{H}$ & 0.12230800 & 1.48319400 & 6.60228800 \\
\hline
\end{tabular}




$\begin{array}{lrrr}\text { C } & -2.78981500 & 1.71180900 & 3.87311300 \\ \mathrm{C} & -3.14792200 & 2.09790700 & 5.17547600 \\ \mathrm{C} & -2.74213400 & 2.67436400 & 2.85935100 \\ \mathrm{C} & -3.46440800 & 3.41870800 & 5.44938100 \\ \mathrm{H} & -3.17290800 & 1.35457900 & 5.96651800 \\ \mathrm{C} & -3.04165900 & 3.99883900 & 3.14891300 \\ \mathrm{H} & -2.44500300 & 2.37905900 & 1.85349700 \\ \mathrm{C} & -3.40880700 & 4.37442900 & 4.43672600 \\ \mathrm{H} & -3.75397800 & 3.70502600 & 6.45743300 \\ \mathrm{H} & -3.01102500 & 4.73540800 & 2.35141300 \\ \mathrm{H} & -3.65355000 & 5.41199100 & 4.65210500 \\ \mathrm{C} & -2.63353200 & 0.30386500 & 3.54564200 \\ \mathrm{H} & -3.01630400 & -0.07554200 & 2.59628700\end{array}$

\begin{tabular}{|c|c|c|c|}
\hline \multicolumn{4}{|c|}{ r-(tx)-A (-5229.580797 a.u.) } \\
\hline $\mathrm{C}$ & -0.86792900 & 2.58923900 & -0.58366500 \\
\hline $\mathrm{C}$ & -2.12687800 & -1.05909700 & -1.04576400 \\
\hline $\mathrm{C}$ & 1.23631300 & -2.17984300 & -1.25948500 \\
\hline $\mathrm{C}$ & 2.55653000 & 1.28516200 & -0.73899400 \\
\hline $\mathrm{O}$ & -1.65290400 & -0.94940300 & 0.13181700 \\
\hline $\mathrm{O}$ & -1.70689900 & -0.46636600 & -2.07645600 \\
\hline $\mathrm{O}$ & 1.11483400 & -1.80131700 & -0.04888200 \\
\hline $\mathrm{O}$ & 0.97914900 & -1.50257200 & -2.28690100 \\
\hline $\mathrm{O}$ & 2.00733000 & 0.97122800 & 0.36316500 \\
\hline $\mathrm{O}$ & 2.06211800 & 1.15946300 & -1.89311300 \\
\hline $\mathrm{O}$ & -0.69231800 & 1.92087300 & 0.48477500 \\
\hline $\mathrm{O}$ & -0.62113100 & 2.21484100 & -1.75850100 \\
\hline $\mathrm{Rh}$ & 0.20100300 & 0.02627100 & 0.34046500 \\
\hline $\mathrm{Rh}$ & 0.17854500 & 0.37275400 & -2.10499400 \\
\hline $\mathrm{C}$ & 1.62423300 & -3.61691100 & -1.53657200 \\
\hline $\mathrm{C}$ & 2.65668800 & -3.79379000 & -2.60888800 \\
\hline $\mathrm{C}$ & 3.01146900 & -4.18924600 & -1.20862800 \\
\hline $\mathrm{C}$ & 0.45618600 & -4.55312300 & -1.39714400 \\
\hline $\mathrm{H}$ & 2.52370100 & -4.56616100 & -3.36210200 \\
\hline $\mathrm{H}$ & 3.08343600 & -2.86706500 & -2.98983300 \\
\hline $\mathrm{C}$ & 4.06164000 & -3.43212200 & -0.45215800 \\
\hline $\mathrm{C}$ & 3.07856900 & -5.66484800 & -0.91182000 \\
\hline $\mathrm{C}$ & -0.40148700 & -4.42664400 & -0.29817500 \\
\hline $\mathrm{C}$ & 0.26138900 & -5.63014800 & -2.26580300 \\
\hline $\mathrm{C}$ & 3.83608200 & -2.96246000 & 0.84228100 \\
\hline $\mathrm{C}$ & 5.35016100 & -3.34710000 & -0.98520300 \\
\hline $\mathrm{C}$ & 2.54132600 & -6.18016700 & 0.26917400 \\
\hline $\mathrm{C}$ & 3.69744500 & -6.54151500 & -1.80158800 \\
\hline $\mathrm{H}$ & -0.30112400 & -3.57022600 & 0.36690300 \\
\hline & & S146 & \\
\hline
\end{tabular}




\begin{tabular}{|c|c|c|c|}
\hline C & -1.36570600 & -5.39473300 & -0.03546000 \\
\hline C & -0.71212800 & -6.58944100 & -2.01031600 \\
\hline $\mathrm{H}$ & 0.89249400 & -5.74232100 & -3.14353300 \\
\hline $\mathrm{H}$ & 2.83277900 & -2.98182800 & 1.25737500 \\
\hline C & 4.88340800 & -2.45103200 & 1.60007100 \\
\hline C & 6.39478500 & -2.81217900 & -0.23896400 \\
\hline $\mathrm{H}$ & 5.53116900 & -3.72314000 & -1.99316200 \\
\hline $\mathrm{H}$ & 2.04841900 & -5.50323900 & 0.96457700 \\
\hline C & 2.58875600 & -7.54108100 & 0.53809700 \\
\hline C & 3.75697900 & -7.90568000 & -1.53204700 \\
\hline $\mathrm{H}$ & 4.12970500 & -6.14985900 & -2.72198500 \\
\hline $\mathrm{H}$ & -2.00027700 & -5.28487300 & 0.84522400 \\
\hline C & -1.51956700 & -6.48881600 & -0.88101600 \\
\hline $\mathrm{H}$ & -0.83200100 & -7.42586900 & -2.69540300 \\
\hline $\mathrm{H}$ & 4.68310200 & -2.10777700 & 2.61320600 \\
\hline C & 6.16673600 & -2.37982700 & 1.06494600 \\
\hline $\mathrm{H}$ & 7.38929600 & -2.73659200 & -0.67516700 \\
\hline $\mathrm{H}$ & 2.14585500 & -7.92634400 & 1.45401300 \\
\hline C & 3.19504300 & -8.41061400 & -0.36513100 \\
\hline $\mathrm{H}$ & 4.23963200 & -8.57564100 & -2.24045400 \\
\hline $\mathrm{H}$ & 6.98362100 & -1.97513300 & 1.66006700 \\
\hline $\mathrm{H}$ & 3.23011500 & -9.47783200 & -0.15796900 \\
\hline C & 3.92715300 & 1.89527100 & -0.69992000 \\
\hline C & 3.95570600 & 3.33738300 & -0.25212500 \\
\hline C & 4.62518100 & 2.32010200 & 0.60203300 \\
\hline C & 4.79167400 & 1.40944800 & -1.82292200 \\
\hline $\mathrm{H}$ & 4.55544700 & 4.04073000 & -0.82227800 \\
\hline $\mathrm{H}$ & 3.00599100 & 3.74287500 & 0.09900700 \\
\hline C & 4.12982000 & 2.07647500 & 1.99749800 \\
\hline C & 6.13036800 & 2.26889400 & 0.55231100 \\
\hline C & 4.93018300 & 0.02775200 & -1.99080800 \\
\hline C & 5.51246200 & 2.25799100 & -2.65789800 \\
\hline C & 3.96857100 & 0.79399600 & 2.51884500 \\
\hline C & 4.00174800 & 3.17377300 & 2.85095700 \\
\hline C & 6.77945400 & 1.03343600 & 0.53258000 \\
\hline C & 6.89722600 & 3.43159900 & 0.53812500 \\
\hline $\mathrm{H}$ & 4.37942300 & -0.65277600 & -1.33406600 \\
\hline C & 5.77849400 & -0.48885900 & -2.95889100 \\
\hline C & 6.37067800 & 1.74247400 & -3.62536800 \\
\hline $\mathrm{H}$ & 5.41407300 & 3.33665800 & -2.54929200 \\
\hline $\mathrm{H}$ & 4.00942600 & -0.06377200 & 1.84902100 \\
\hline C & 3.73379400 & 0.60917200 & 3.87557900 \\
\hline C & 3.76206200 & 2.99439100 & 4.20960900 \\
\hline $\mathrm{H}$ & 4.12427100 & 4.17777800 & 2.44402600 \\
\hline
\end{tabular}




\begin{tabular}{|c|c|c|c|}
\hline $\mathrm{H}$ & 6.18482300 & 0.12096200 & 0.54565800 \\
\hline C & 8.16293200 & 0.96103900 & 0.45711000 \\
\hline C & 8.28683500 & 3.36332900 & 0.47790900 \\
\hline $\mathrm{H}$ & 6.39913500 & 4.40065800 & 0.56705300 \\
\hline $\mathrm{H}$ & 5.87596600 & -1.56834300 & -3.06202400 \\
\hline C & 6.50898300 & 0.36837400 & -3.77844900 \\
\hline $\mathrm{H}$ & 6.93313400 & 2.42223800 & -4.26190100 \\
\hline $\mathrm{H}$ & 3.59168300 & -0.39893200 & 4.25681000 \\
\hline C & 3.65142500 & 1.70742700 & 4.72821200 \\
\hline $\mathrm{H}$ & 3.68422800 & 3.85940700 & 4.86590200 \\
\hline $\mathrm{H}$ & 8.65077600 & -0.01168400 & 0.41451700 \\
\hline C & 8.92309500 & 2.12832000 & 0.42717200 \\
\hline $\mathrm{H}$ & 8.87283300 & 4.28005200 & 0.46328000 \\
\hline $\mathrm{H}$ & 3.49607900 & 1.55886800 & 5.79609700 \\
\hline $\mathrm{H}$ & 10.00778100 & 2.07415700 & 0.36462000 \\
\hline C & -1.51226000 & 3.94939700 & -0.47120800 \\
\hline C & -0.97130800 & 4.88203500 & 0.57193500 \\
\hline C & -0.69963600 & 5.19545100 & -0.86885400 \\
\hline C & -2.97301300 & 3.92587000 & -0.84323800 \\
\hline $\mathrm{H}$ & -1.65097900 & 5.51834500 & 1.13450300 \\
\hline H & -0.13922700 & 4.48903600 & 1.15707100 \\
\hline C & 0.71939300 & 5.19034600 & -1.37421500 \\
\hline C & -1.48291800 & 6.30207800 & -1.52091400 \\
\hline C & -3.45110600 & 3.01970700 & -1.80000000 \\
\hline C & -3.88046500 & 4.85554700 & -0.32028000 \\
\hline C & 1.14405500 & 4.46467200 & -2.48948800 \\
\hline C & 1.59703000 & 6.12033400 & -0.80359700 \\
\hline C & -1.95349000 & 6.13289000 & -2.82512300 \\
\hline C & -1.73635800 & 7.50328700 & -0.86230400 \\
\hline $\mathrm{H}$ & -2.77737700 & 2.28461200 & -2.23632400 \\
\hline C & -4.77951100 & 3.04925900 & -2.21387900 \\
\hline C & -5.20400600 & 4.89303800 & -0.74331300 \\
\hline $\mathrm{H}$ & -3.54681500 & 5.59957500 & 0.39911200 \\
\hline H & 0.48493100 & 3.72582000 & -2.93493300 \\
\hline C & 2.41419200 & 4.67129800 & -3.02156600 \\
\hline C & 2.85300900 & 6.34702900 & -1.35114900 \\
\hline H & 1.26791700 & 6.69351900 & 0.06346300 \\
\hline $\mathrm{H}$ & -1.75505900 & 5.19291200 & -3.34048700 \\
\hline C & -2.69485200 & 7.13051900 & -3.44272400 \\
\hline C & -2.47362200 & 8.50883400 & -1.48174500 \\
\hline $\mathrm{H}$ & -1.36139000 & 7.64866200 & 0.15097400 \\
\hline H & -5.11845900 & 2.32465400 & -2.95288900 \\
\hline C & -5.66336100 & 3.98736700 & -1.69409600 \\
\hline $\mathrm{H}$ & -5.87633500 & 5.63938900 & -0.32643500 \\
\hline
\end{tabular}




\begin{tabular}{|c|c|c|c|}
\hline $\mathrm{H}$ & 2.73713400 & 4.07684500 & -3.87364600 \\
\hline C & 3.26143200 & 5.62616500 & -2.47092200 \\
\hline $\mathrm{H}$ & 3.51383100 & 7.08683100 & -0.90389100 \\
\hline $\mathrm{H}$ & -3.07131100 & 6.97778800 & -4.45163000 \\
\hline C & -2.96145500 & 8.32121000 & -2.76978800 \\
\hline $\mathrm{H}$ & -2.66925100 & 9.43976800 & -0.95372000 \\
\hline $\mathrm{H}$ & 4.24521300 & 5.80005400 & -2.90429600 \\
\hline $\mathrm{H}$ & -3.54585600 & 9.10172600 & -3.25174700 \\
\hline C & -3.25702700 & -2.03369600 & -1.28731100 \\
\hline C & -3.59985700 & -2.98564000 & -0.17839400 \\
\hline C & -4.60818600 & -1.94467300 & -0.53573100 \\
\hline C & -3.32132600 & -2.51899600 & -2.70504900 \\
\hline $\mathrm{H}$ & -3.77183600 & -4.02495000 & -0.44594600 \\
\hline $\mathrm{H}$ & -3.04017000 & -2.83733000 & 0.74418100 \\
\hline C & -4.90285300 & -0.84841100 & 0.43833000 \\
\hline C & -5.82370700 & -2.31982000 & -1.34485100 \\
\hline C & -3.86545300 & -1.70328100 & -3.69962300 \\
\hline C & -2.85512300 & -3.78409900 & -3.04754700 \\
\hline C & -4.60570500 & 0.48624200 & 0.16860900 \\
\hline C & -5.65254800 & -1.15366400 & 1.57826700 \\
\hline C & -6.65119000 & -1.30576700 & -1.84051900 \\
\hline C & -6.13175500 & -3.63985900 & -1.67633400 \\
\hline $\mathrm{H}$ & -4.21710400 & -0.70666800 & -3.43125400 \\
\hline C & -3.96984400 & -2.15797200 & -5.00563200 \\
\hline C & -2.94784400 & -4.23974800 & -4.36029800 \\
\hline $\mathrm{H}$ & -2.42133600 & -4.42480200 & -2.28185000 \\
\hline $\mathrm{H}$ & -4.04109700 & 0.74028100 & -0.72893300 \\
\hline C & -5.06401700 & 1.49748300 & 1.00850700 \\
\hline C & -6.08010000 & -0.14901800 & 2.44175200 \\
\hline $\mathrm{H}$ & -5.91640000 & -2.19582700 & 1.76832100 \\
\hline $\mathrm{H}$ & -6.43691900 & -0.26822100 & -1.58576700 \\
\hline C & -7.72808700 & -1.59945100 & -2.66623800 \\
\hline C & -7.20967500 & -3.93706500 & -2.50307400 \\
\hline $\mathrm{H}$ & -5.51405400 & -4.45478400 & -1.30649300 \\
\hline $\mathrm{H}$ & -4.40922900 & -1.51849900 & -5.76806300 \\
\hline C & -3.51240700 & -3.43245300 & -5.33968800 \\
\hline $\mathrm{H}$ & -2.57648300 & -5.23264200 & -4.60809300 \\
\hline $\mathrm{H}$ & -4.84352400 & 2.53570200 & 0.76612200 \\
\hline C & -5.79402200 & 1.18222700 & 2.15072100 \\
\hline $\mathrm{H}$ & -6.65541200 & -0.40491900 & 3.32910900 \\
\hline $\mathrm{H}$ & -8.34878300 & -0.79153600 & -3.04769400 \\
\hline C & -8.00963600 & -2.91866000 & -3.00737900 \\
\hline $\mathrm{H}$ & -7.42068600 & -4.97382200 & -2.75559800 \\
\hline $\mathrm{H}$ & -6.12733300 & 1.97556300 & 2.81760800 \\
\hline
\end{tabular}




\begin{tabular}{|c|c|c|c|}
\hline $\mathrm{H}$ & -8.84880600 & -3.15096100 & -3.65894200 \\
\hline $\mathrm{H}$ & -6.69881700 & 4.01347000 & -2.02465900 \\
\hline $\mathrm{H}$ & -3.59594300 & -3.79010500 & -6.36375500 \\
\hline $\mathrm{H}$ & -2.26795800 & -7.24959500 & -0.67069500 \\
\hline $\mathrm{H}$ & 7.18170500 & -0.03454800 & -4.53230800 \\
\hline C & 0.27350600 & -0.28658900 & 2.36738200 \\
\hline C & 0.80105200 & -1.60695100 & 2.75725200 \\
\hline O & -0.04843900 & -2.62521300 & 2.52381200 \\
\hline C & 0.48245200 & -3.90514600 & 2.85273200 \\
\hline$H$ & 0.71371900 & -3.96684900 & 3.92147000 \\
\hline $\mathrm{H}$ & 1.40051100 & -4.09772300 & 2.28862100 \\
\hline $\mathrm{H}$ & -0.28772200 & -4.63272400 & 2.58853600 \\
\hline O & 1.91749800 & -1.78857700 & 3.20556000 \\
\hline 0 & -1.36550300 & -0.11437700 & 5.33151500 \\
\hline N & -2.15744600 & -0.63598500 & 4.37022100 \\
\hline C & -2.28973600 & -2.04861100 & 4.35424500 \\
\hline C & -2.61195700 & -4.80303100 & 4.38723600 \\
\hline C & -1.57849200 & -2.80845000 & 5.28003200 \\
\hline C & -3.17620600 & -2.64690000 & 3.46157700 \\
\hline C & -1.75371500 & -4.18676100 & 5.29368200 \\
\hline C & -3.31663100 & -4.02681000 & 3.46956400 \\
\hline $\mathrm{H}$ & -0.90626800 & -2.31754700 & 5.97649500 \\
\hline $\mathrm{H}$ & -3.76876100 & -2.04588000 & 2.77737900 \\
\hline $\mathrm{H}$ & -1.20436200 & -4.78420900 & 6.01716800 \\
\hline $\mathrm{H}$ & -4.00371900 & -4.49053700 & 2.76335300 \\
\hline C & -0.01243800 & 0.42301200 & 4.76447600 \\
\hline $\mathrm{H}$ & 0.68635100 & -0.38412300 & 5.02324600 \\
\hline H & -2.73958000 & -5.88240000 & 4.40224700 \\
\hline C & -0.04093300 & 0.65006500 & 3.29938600 \\
\hline $\mathrm{H}$ & -0.25994100 & 1.66477300 & 2.95750200 \\
\hline C & 0.21322500 & 1.66513700 & 5.58251300 \\
\hline $\mathrm{H}$ & 1.21648700 & 2.05078700 & 5.37354000 \\
\hline $\mathrm{H}$ & -0.51712600 & 2.43724700 & 5.31142100 \\
\hline $\mathrm{H}$ & 0.12585300 & 1.45810300 & 6.65382000 \\
\hline C & -2.88763400 & 1.59090100 & 3.77281600 \\
\hline C & -3.39029900 & 1.89751900 & 5.04899700 \\
\hline C & -2.83819300 & 2.58563400 & 2.79064400 \\
\hline C & -3.83716900 & 3.17760900 & 5.33191500 \\
\hline $\mathrm{H}$ & -3.43686800 & 1.12478800 & 5.81077900 \\
\hline C & -3.26983300 & 3.86962700 & 3.09221900 \\
\hline $\mathrm{H}$ & -2.43314900 & 2.34860700 & 1.80678400 \\
\hline C & -3.77276000 & 4.16870000 & 4.35454800 \\
\hline H & -4.23750900 & 3.40343400 & 6.31689200 \\
\hline $\mathrm{H}$ & -3.23527400 & 4.63442200 & 2.32186000 \\
\hline
\end{tabular}




$\begin{array}{lrrr}H & -4.11957400 & 5.17519100 & 4.57654700 \\ \mathrm{C} & -2.57543000 & 0.20993700 & 3.44544900 \\ \mathrm{H} & -2.86078700 & -0.20656700 & 2.47354500\end{array}$

r-TS(tx)-II (-5229.580169 a.u.)

\begin{tabular}{|c|c|c|c|}
\hline C & -0.97009300 & 2.53629700 & -0.62024700 \\
\hline C & -2.07713500 & -1.15699100 & -1.08650700 \\
\hline C & 1.31652800 & -2.15285900 & -1.26632800 \\
\hline C & 2.50770500 & 1.36610600 & -0.74667600 \\
\hline $\mathrm{O}$ & -1.61709000 & -1.00940800 & 0.09279600 \\
\hline $\mathrm{O}$ & -1.65475100 & -0.58370400 & -2.12706600 \\
\hline 0 & 1.15902400 & -1.78042200 & -0.05721200 \\
\hline $\mathrm{O}$ & 1.07384600 & -1.47381800 & -2.29593200 \\
\hline $\mathrm{O}$ & 1.96596200 & 1.02582700 & 0.35213800 \\
\hline 0 & 2.01526600 & 1.23778400 & -1.90106700 \\
\hline $\mathrm{O}$ & -0.76196000 & 1.88088400 & 0.45155100 \\
\hline 0 & -0.72730200 & 2.15527900 & -1.79356200 \\
\hline $\mathrm{Rh}$ & 0.18856800 & 0.02047400 & 0.31700600 \\
\hline $\mathrm{Rh}$ & 0.17720300 & 0.35650000 & -2.12562000 \\
\hline C & 1.73779800 & -3.58147200 & -1.53627300 \\
\hline C & 2.78631200 & -3.73793000 & -2.59597100 \\
\hline C & 3.13595800 & -4.11443400 & -1.18925600 \\
\hline C & 0.59160200 & -4.54612800 & -1.40380900 \\
\hline $\mathrm{H}$ & 2.68148600 & -4.51961300 & -3.34386900 \\
\hline $\mathrm{H}$ & 3.19231800 & -2.80333900 & -2.98013100 \\
\hline C & 4.15275600 & -3.32025700 & -0.42512700 \\
\hline C & 3.24143100 & -5.58509800 & -0.88038500 \\
\hline C & -0.27130300 & -4.44293700 & -0.30675300 \\
\hline C & 0.42555500 & -5.62656300 & -2.27428500 \\
\hline C & 3.89791900 & -2.85703200 & 0.86648800 \\
\hline C & 5.44217800 & -3.18859700 & -0.94606800 \\
\hline C & 2.70609900 & -6.10655900 & 0.29877500 \\
\hline C & 3.89707500 & -6.44973800 & -1.75544600 \\
\hline $\mathrm{H}$ & -0.19271500 & -3.58566100 & 0.35923800 \\
\hline C & -1.21067800 & -5.43509100 & -0.04509900 \\
\hline C & -0.52342700 & -6.61073500 & -2.02009700 \\
\hline $\mathrm{H}$ & 1.06068000 & -5.72209400 & -3.15118800 \\
\hline $\mathrm{H}$ & 2.89211200 & -2.91405900 & 1.27244200 \\
\hline C & 4.91776900 & -2.30498000 & 1.63296600 \\
\hline C & 6.45945100 & -2.61562400 & -0.19015900 \\
\hline $\mathrm{H}$ & 5.64665300 & -3.55897600 & -1.95160600 \\
\hline $\mathrm{H}$ & 2.18587700 & -5.43904700 & 0.98326300 \\
\hline C & 2.79215900 & -7.46299100 & 0.58040500 \\
\hline C & 3.99571000 & -7.80898200 & -1.47277700 \\
\hline
\end{tabular}


$\mathrm{H}$

$\mathrm{H}$

C

$\mathrm{H}$

$\mathrm{H}$

C

$\mathrm{H}$

$\mathrm{H}$

C

$\mathrm{H}$

$\mathrm{H}$

$\mathrm{H}$

C

C

C

C

$\mathrm{H}$

$\mathrm{H}$

C

C

C

C

C

C

C

C

$\mathrm{H}$

C

C

$\mathrm{H}$

$\mathrm{H}$

C

C

$\mathrm{H}$

$\mathrm{H}$

C

C

$\mathrm{H}$

$\mathrm{H}$

C

$\mathrm{H}$

$\mathrm{H}$

C

$\mathrm{H}$

$\begin{array}{ccc}4.32852500 & -6.05251700 & -2.67384000 \\ -1.84937500 & -5.34159800 & 0.83458700 \\ -1.33429800 & -6.53232900 & -0.89144900 \\ -0.62086000 & -7.44950500 & -2.70584200 \\ 4.69523800 & -1.96425400 & 2.64231000 \\ 6.20302400 & -2.18926800 & 1.11050300 \\ 7.45495500 & -2.50541100 & -0.61665600 \\ 2.35093400 & -7.85386400 & 1.49472300 \\ 3.43604600 & -8.32089200 & -0.30779300 \\ 4.50736600 & -8.46976200 & -2.16930400 \\ 6.99870600 & -1.75373400 & 1.71249600 \\ 3.50199700 & -9.38460000 & -0.09044800 \\ 3.86272500 & 2.00981400 & -0.70657000 \\ 3.85826500 & 3.45260900 & -0.25925700 \\ 4.55933900 & 2.45248700 & 0.59010000 \\ 4.73528600 & 1.54363500 & -1.83232400 \\ 4.43514500 & 4.17064200 & -0.83482100 \\ 2.90114100 & 3.83386800 & 0.09889400 \\ 4.08487400 & 2.19549200 & 1.99035900 \\ 6.06501600 & 2.43504500 & 0.52684600 \\ 4.92171700 & 0.16609500 & -1.98732500 \\ 5.41811900 & 2.40913300 & -2.68138500 \\ 3.91128200 & 0.90969800 & 2.49946200 \\ 3.99239500 & 3.28463100 & 2.85932300 \\ 6.73711200 & 1.21203400 & 0.52629300 \\ 6.80939400 & 3.61131200 & 0.48020200 \\ 4.40071100 & -0.52755400 & -1.31985900 \\ 5.78177000 & -0.32956400 & -2.95607600 \\ 6.28743300 & 1.91480400 & -3.65007600 \\ 5.27976900 & 3.48450300 & -2.58331600 \\ 3.93170500 & 0.05908300 & 1.81964800 \\ 3.69422100 & 0.71379100 & 3.85838200 \\ 3.77339000 & 3.09385800 & 4.21967500 \\ 4.12671200 & 4.29088200 & 2.46164800 \\ 6.15919000 & 0.28972800 & 0.56479000 \\ 8.12060700 & 1.16308300 & 0.43774700 \\ 8.19954600 & 3.56708900 & 0.40707000 \\ 6.29440200 & 4.57178400 & 0.49302800 \\ 5.91862200 & -1.40577400 & -3.04837300 \\ 6.47496600 & 0.54511200 & -3.78949800 \\ 6.82004900 & 2.60758700 & -4.29809200 \\ 3.54188800 & -0.29618100 & 4.23117100 \\ 3.64503400 & 1.80300400 & 4.72484200 \\ 3.72211700 & 3.95225600 & 4.88698100\end{array}$




\begin{tabular}{|c|c|c|c|}
\hline $\mathrm{H}$ & 8.62553600 & 0.19856100 & 0.41063700 \\
\hline C & 8.85843200 & 2.34343300 & 0.37568900 \\
\hline $\mathrm{H}$ & 8.76806400 & 4.49397200 & 0.36712700 \\
\hline $\mathrm{H}$ & 3.49620200 & 1.64497600 & 5.79219900 \\
\hline $\mathrm{H}$ & 9.94325000 & 2.30827900 & 0.30317100 \\
\hline C & -1.64245100 & 3.88229300 & -0.50250300 \\
\hline C & -1.11055600 & 4.82414900 & 0.53728700 \\
\hline C & -0.86506900 & 5.14897800 & -0.90560100 \\
\hline C & -3.10735000 & 3.82936200 & -0.85420500 \\
\hline $\mathrm{H}$ & -1.79834500 & 5.44102600 & 1.11127700 \\
\hline $\mathrm{H}$ & -0.26026600 & 4.45163300 & 1.10951700 \\
\hline C & 0.54779200 & 5.17919500 & -1.42591400 \\
\hline C & -1.68548200 & 6.23695900 & -1.54301900 \\
\hline C & -3.57330800 & 2.93930500 & -1.83123400 \\
\hline C & -4.03196700 & 4.71496000 & -0.28785600 \\
\hline C & 0.98197200 & 4.45474300 & -2.53776700 \\
\hline C & 1.40667300 & 6.13489800 & -0.86899900 \\
\hline C & -2.16181100 & 6.06304800 & -2.84442300 \\
\hline C & -1.96962700 & 7.42485900 & -0.87307700 \\
\hline $\mathrm{H}$ & -2.88290500 & 2.24221100 & -2.30182600 \\
\hline C & -4.90931200 & 2.93734000 & -2.21987500 \\
\hline C & -5.36335700 & 4.72297600 & -0.68698700 \\
\hline $\mathrm{H}$ & -3.70954500 & 5.44218200 & 0.45325600 \\
\hline $\mathrm{H}$ & 0.33619900 & 3.69884000 & -2.97426100 \\
\hline C & 2.24558300 & 4.68277700 & -3.07699700 \\
\hline C & 2.65418300 & 6.38461200 & -1.42532100 \\
\hline $\mathrm{H}$ & 1.06907700 & 6.70840500 & -0.00542700 \\
\hline $\mathrm{H}$ & -1.93917900 & 5.13350700 & -3.36872600 \\
\hline C & -2.93744900 & 7.04259200 & -3.44866300 \\
\hline C & -2.74119500 & 8.41257800 & -1.47921300 \\
\hline $\mathrm{H}$ & -1.59154900 & 7.57372200 & 0.13851000 \\
\hline $\mathrm{H}$ & -5.23930200 & 2.22519500 & -2.97460100 \\
\hline C & -5.81184100 & 3.83065400 & -1.65522000 \\
\hline $\mathrm{H}$ & -6.05173600 & 5.43307100 & -0.23458900 \\
\hline $\mathrm{H}$ & 2.57819100 & 4.08627400 & -3.92387600 \\
\hline C & 3.07428100 & 5.66058000 & -2.53896700 \\
\hline $\mathrm{H}$ & 3.30023600 & 7.14368600 & -0.98888500 \\
\hline $\mathrm{H}$ & -3.31756900 & 6.88603300 & -4.45560000 \\
\hline C & -3.23348500 & 8.22000900 & -2.76477600 \\
\hline $\mathrm{H}$ & -2.96028100 & 9.33326800 & -0.94256700 \\
\hline $\mathrm{H}$ & 4.05236400 & 5.85133000 & -2.97824500 \\
\hline $\mathrm{H}$ & -3.84463900 & 8.98627900 & -3.23626800 \\
\hline C & -3.19276600 & -2.14805300 & -1.30356700 \\
\hline C & -3.50225900 & -3.09419500 & -0.17847300 \\
\hline
\end{tabular}




\begin{tabular}{|c|c|c|c|}
\hline C & -4.52929100 & -2.06837000 & -0.52488200 \\
\hline C & -3.27636700 & -2.64385100 & -2.71597800 \\
\hline $\mathrm{H}$ & -3.66668600 & -4.13767200 & -0.43478400 \\
\hline $\mathrm{H}$ & -2.92743900 & -2.92966600 & 0.73273700 \\
\hline C & -4.81938500 & -0.97105800 & 0.44979800 \\
\hline C & -5.75357700 & -2.46142600 & -1.31118200 \\
\hline C & -3.84660000 & -1.84027900 & -3.70574100 \\
\hline C & -2.80163100 & -3.90591900 & -3.05824700 \\
\hline C & -4.50994200 & 0.36328400 & 0.19389800 \\
\hline C & -5.57523400 & -1.28161300 & 1.58412800 \\
\hline C & -6.59857500 & -1.45699700 & -1.79668000 \\
\hline C & -6.05383600 & -3.78565600 & -1.63212300 \\
\hline $\mathrm{H}$ & -4.20520700 & -0.84602400 & -3.43695900 \\
\hline C & -3.96855300 & -2.30471900 & -5.00686100 \\
\hline C & -2.91201000 & -4.37103200 & -4.36619900 \\
\hline $\mathrm{H}$ & -2.34705400 & -4.53626300 & -2.29575400 \\
\hline $\mathrm{H}$ & -3.93772000 & 0.62480800 & -0.69711200 \\
\hline C & -4.96459700 & 1.36904100 & 1.04208100 \\
\hline C & -6.00227000 & -0.28262600 & 2.45330400 \\
\hline $\mathrm{H}$ & -5.84169900 & -2.32424600 & 1.76699800 \\
\hline H & -6.38903900 & -0.41690800 & -1.54807500 \\
\hline C & -7.68622900 & -1.76418200 & -2.60304100 \\
\hline C & -7.14247800 & -4.09636700 & -2.43976800 \\
\hline H & -5.42198800 & -4.59286600 & -1.26916400 \\
\hline $\mathrm{H}$ & -4.42850900 & -1.67543500 & -5.76559800 \\
\hline C & -3.50288900 & -3.57630000 & -5.34051500 \\
\hline $\mathrm{H}$ & -2.53387600 & -5.36119500 & -4.61467000 \\
\hline $\mathrm{H}$ & -4.73650900 & 2.40767700 & 0.81052600 \\
\hline C & -5.70607400 & 1.04885000 & 2.17520000 \\
\hline $\mathrm{H}$ & -6.58327300 & -0.54281100 & 3.33560300 \\
\hline $\mathrm{H}$ & -8.32135400 & -0.96395500 & -2.97688200 \\
\hline C & -7.96049200 & -3.08756200 & -2.93434000 \\
\hline $\mathrm{H}$ & -7.34802800 & -5.13607000 & -2.68460900 \\
\hline H & -6.04679000 & 1.83858600 & 2.84219800 \\
\hline $\mathrm{H}$ & -8.80833300 & -3.33059000 & -3.57059800 \\
\hline $\mathrm{H}$ & -6.85400200 & 3.83265200 & -1.96505700 \\
\hline H & -3.60052800 & -3.94169400 & -6.36059000 \\
\hline $\mathrm{H}$ & -2.06252800 & -7.31277400 & -0.68256400 \\
\hline $\mathrm{H}$ & 7.15702600 & 0.15900700 & -4.54371800 \\
\hline C & 0.21975500 & -0.28511200 & 2.32748800 \\
\hline C & 0.78081200 & -1.58841500 & 2.73558700 \\
\hline $\mathrm{O}$ & -0.02239000 & -2.63555000 & 2.49891000 \\
\hline C & 0.54871000 & -3.89303000 & 2.85550100 \\
\hline $\mathrm{H}$ & 0.76221800 & -3.92810800 & 3.92854200 \\
\hline
\end{tabular}




\begin{tabular}{lccc}
$\mathrm{H}$ & 1.48084500 & -4.05989800 & 2.30669500 \\
$\mathrm{H}$ & -0.19307400 & -4.64917000 & 2.59168700 \\
$\mathrm{O}$ & 1.89950500 & -1.71161300 & 3.19764400 \\
$\mathrm{O}$ & -1.25644100 & -0.11342100 & 5.38417100 \\
$\mathrm{~N}$ & -2.09094000 & -0.64049700 & 4.44949800 \\
$\mathrm{C}$ & -2.19904100 & -2.04771000 & 4.41253800 \\
$\mathrm{C}$ & -2.49660500 & -4.81034100 & 4.40182200 \\
$\mathrm{C}$ & -1.49683900 & -2.81738200 & 5.33910500 \\
$\mathrm{C}$ & -3.06399800 & -2.64503400 & 3.49586100 \\
$\mathrm{C}$ & -1.65986300 & -4.19747100 & 5.33053500 \\
$\mathrm{C}$ & -3.19139800 & -4.02629900 & 3.48303600 \\
$\mathrm{H}$ & -0.84196100 & -2.33033100 & 6.05469500 \\
$\mathrm{H}$ & -3.64550800 & -2.03964300 & 2.80522400 \\
$\mathrm{H}$ & -1.11754900 & -4.79950900 & 6.05590800 \\
$\mathrm{H}$ & -3.86059600 & -4.48671600 & 2.75740600 \\
$\mathrm{C}$ & 0.00085100 & 0.46898100 & 4.73976900 \\
$\mathrm{H}$ & 0.77793300 & -0.27693500 & 4.95748300 \\
$\mathrm{H}$ & -2.61537700 & -5.89088000 & 4.40054900 \\
$\mathrm{C}$ & -0.15452500 & 0.64977600 & 3.26369400 \\
$\mathrm{H}$ & -0.31385600 & 1.66938700 & 2.90515400 \\
$\mathrm{C}$ & 0.23121200 & 1.75830700 & 5.48338400 \\
$\mathrm{H}$ & 1.17850500 & 2.19945700 & 5.15662800 \\
$\mathrm{H}$ & -0.57683700 & 2.46953700 & 5.27418700 \\
$\mathrm{H}$ & 0.27458200 & 1.58640000 & 6.56361700 \\
$\mathrm{C}$ & -2.83369600 & 1.57306300 & 3.81487400 \\
$\mathrm{C}$ & -3.41050900 & 1.80106100 & 5.07315000 \\
$\mathrm{C}$ & -2.80054000 & 2.60807400 & 2.87585000 \\
$\mathrm{C}$ & -3.93603600 & 3.04541200 & 5.38692800 \\
$\mathrm{H}$ & -3.44862700 & 0.99601300 & 5.80127000 \\
$\mathrm{C}$ & -3.32127300 & 3.85249200 & 3.20260700 \\
$\mathrm{H}$ & -2.34648600 & 2.43322100 & 1.90040400 \\
$\mathrm{C}$ & -3.88809200 & 4.07747700 & 4.45370100 \\
$\mathrm{H}$ & -4.38608100 & 3.20866100 & 6.36296500 \\
$\mathrm{H}$ & -3.29897600 & 4.64831400 & 2.46289700 \\
$\mathrm{H}$ & -4.29669600 & 5.05493300 & 4.69895600 \\
$\mathrm{H}$ & -2.38941500 & 0.21927100 & 3.46689900 \\
& & -0.20440900 & 2.49577000 \\
\hline
\end{tabular}

$\begin{array}{lrrr}\text { r-(tx)-B } & & & \\ \text { C } & -1.11614000 & 2.41125800 & -0.65095100 \\ C & -2.04759200 & -1.33888600 & -1.10633500 \\ C & 1.40054700 & -2.16010000 & -1.23281000 \\ C & 2.38788700 & 1.42643100 & -0.78591600 \\ O & -1.53666300 & -1.21760900 & 0.05640300 \\ & & \end{array}$




\begin{tabular}{|c|c|c|c|}
\hline $\mathrm{O}$ & -1.68118700 & -0.71633100 & -2.14064300 \\
\hline 0 & 1.27489400 & -1.74854900 & -0.02980000 \\
\hline $\mathrm{O}$ & 1.07499800 & -1.53177700 & -2.27094600 \\
\hline $\mathrm{O}$ & 1.83421100 & 1.12764300 & 0.32300600 \\
\hline 0 & 1.91763800 & 1.20548700 & -1.93444300 \\
\hline $\mathrm{O}$ & -0.92537200 & 1.74577000 & 0.42072300 \\
\hline $\mathrm{O}$ & -0.84467500 & 2.03530900 & -1.81905400 \\
\hline $\mathrm{Rh}$ & 0.15026500 & -0.03699300 & 0.31245800 \\
\hline $\mathrm{Rh}$ & 0.11553300 & 0.26486500 & -2.12271800 \\
\hline C & 1.88575200 & -3.57051600 & -1.46984800 \\
\hline C & 2.92935400 & -3.70525600 & -2.53773200 \\
\hline C & 3.31020500 & -4.02549300 & -1.12488400 \\
\hline C & 0.77624700 & -4.57467300 & -1.31142700 \\
\hline $\mathrm{H}$ & 2.85030500 & -4.51080000 & -3.26317500 \\
\hline $\mathrm{H}$ & 3.29068300 & -2.76403300 & -2.95044000 \\
\hline C & 4.30266400 & -3.15703700 & -0.40965000 \\
\hline C & 3.49029600 & -5.47902400 & -0.77406700 \\
\hline C & -0.10137400 & -4.46646400 & -0.22680400 \\
\hline C & 0.65474100 & -5.68545300 & -2.15023800 \\
\hline C & 4.07427700 & -2.69330000 & 0.88703700 \\
\hline C & 5.55615300 & -2.94496500 & -0.98882000 \\
\hline C & 2.95665400 & -6.00194800 & 0.40487800 \\
\hline C & 4.21905800 & -6.32306900 & -1.61073800 \\
\hline $\mathrm{H}$ & -0.06042200 & -3.59111900 & 0.41732600 \\
\hline C & -1.01643100 & -5.47589000 & 0.05404200 \\
\hline C & -0.26734100 & -6.68958200 & -1.87576700 \\
\hline $\mathrm{H}$ & 1.30389500 & -5.78970900 & -3.01583600 \\
\hline $\mathrm{H}$ & 3.09405500 & -2.81657100 & 1.33943700 \\
\hline C & 5.08669400 & -2.06017700 & 1.59915100 \\
\hline C & 6.56507300 & -2.29453800 & -0.28621400 \\
\hline $\mathrm{H}$ & 5.74124400 & -3.31382600 & -1.99847500 \\
\hline $\mathrm{H}$ & 2.37450700 & -5.35242200 & 1.05573500 \\
\hline C & 3.12070100 & -7.34241800 & 0.72638000 \\
\hline C & 4.39535500 & -7.66524200 & -1.28781400 \\
\hline $\mathrm{H}$ & 4.64695100 & -5.92354100 & -2.52988500 \\
\hline $\mathrm{H}$ & -1.66657300 & -5.37280300 & 0.92400000 \\
\hline C & -1.09584300 & -6.60076400 & -0.76066100 \\
\hline $\mathrm{H}$ & -0.33026700 & -7.55195400 & -2.53568000 \\
\hline $\mathrm{H}$ & 4.89157400 & -1.71784000 & 2.61355400 \\
\hline C & 6.33735400 & -1.86643000 & 1.01858300 \\
\hline $\mathrm{H}$ & 7.53148100 & -2.12513700 & -0.75771400 \\
\hline $\mathrm{H}$ & 2.68073200 & -7.73658700 & 1.63977300 \\
\hline C & 3.84065800 & -8.18013700 & -0.12152400 \\
\hline $\mathrm{H}$ & 4.96356600 & -8.31102900 & -1.95378400 \\
\hline
\end{tabular}




\begin{tabular}{|c|c|c|c|}
\hline $\mathrm{H}$ & 7.12756300 & -1.37189600 & 1.58063600 \\
\hline $\mathrm{H}$ & 3.96805600 & -9.23125700 & 0.12709300 \\
\hline C & 3.70216100 & 2.14295700 & -0.76988400 \\
\hline C & 3.60384500 & 3.58963600 & -0.33851800 \\
\hline C & 4.39309800 & 2.65307200 & 0.50391400 \\
\hline C & 4.57663900 & 1.72061200 & -1.91183600 \\
\hline $\mathrm{H}$ & 4.11219400 & 4.33728400 & -0.93987300 \\
\hline $\mathrm{H}$ & 2.62921000 & 3.90457000 & 0.03582900 \\
\hline C & 3.98052100 & 2.37631200 & 1.91965800 \\
\hline C & 5.89463400 & 2.74946200 & 0.40293500 \\
\hline C & 4.86196700 & 0.35783900 & -2.04657400 \\
\hline C & 5.15541400 & 2.61703600 & -2.80459200 \\
\hline C & 3.85989000 & 1.08588000 & 2.43150300 \\
\hline C & 3.91053800 & 3.46132900 & 2.79743500 \\
\hline C & 6.66363000 & 1.58495600 & 0.42499100 \\
\hline C & 6.54002200 & 3.98016400 & 0.31024400 \\
\hline $\mathrm{H}$ & 4.42035500 & -0.36016500 & -1.34745200 \\
\hline C & 5.71541600 & -0.09217100 & -3.04301000 \\
\hline C & 6.01920100 & 2.16854100 & -3.80023300 \\
\hline $\mathrm{H}$ & 4.93504200 & 3.68004500 & -2.72280900 \\
\hline H & 3.87187300 & 0.23581000 & 1.75012800 \\
\hline C & 3.72349800 & 0.88375700 & 3.80138100 \\
\hline C & 3.77014500 & 3.26273000 & 4.16638400 \\
\hline H & 4.00404900 & 4.47099000 & 2.39686800 \\
\hline $\mathrm{H}$ & 6.16301400 & 0.62044800 & 0.50131000 \\
\hline C & 8.04554400 & 1.64666900 & 0.31704100 \\
\hline C & 7.92769600 & 4.04716300 & 0.21680900 \\
\hline $\mathrm{H}$ & 5.95028400 & 4.89648200 & 0.30519800 \\
\hline $\mathrm{H}$ & 5.92829900 & -1.15687700 & -3.12388200 \\
\hline C & 6.30363200 & 0.81408400 & -3.92239400 \\
\hline $\mathrm{H}$ & 6.46960600 & 2.88492000 & -4.48384400 \\
\hline $\mathrm{H}$ & 3.62484300 & -0.12990800 & 4.18192700 \\
\hline C & 3.69661400 & 1.96830800 & 4.67341900 \\
\hline H & 3.73246300 & 4.11802800 & 4.83815600 \\
\hline $\mathrm{H}$ & 8.62795900 & 0.72660500 & 0.31029300 \\
\hline C & 8.68368700 & 2.88064000 & 0.21082400 \\
\hline H & 8.41786800 & 5.01559500 & 0.14221500 \\
\hline $\mathrm{H}$ & 3.59932500 & 1.80448800 & 5.74539100 \\
\hline $\mathrm{H}$ & 9.76676900 & 2.93136600 & 0.12435100 \\
\hline C & -1.80576300 & 3.74510800 & -0.52625100 \\
\hline C & -1.25656400 & 4.70845800 & 0.48380000 \\
\hline C & -1.09060800 & 5.03398100 & -0.97078200 \\
\hline C & -3.28099300 & 3.64420100 & -0.81494800 \\
\hline $\mathrm{H}$ & -1.93680600 & 5.29873500 & 1.09393500 \\
\hline
\end{tabular}




\begin{tabular}{|c|c|c|c|}
\hline $\mathrm{H}$ & -0.36333700 & 4.37124400 & 1.01192400 \\
\hline C & 0.29555700 & 5.11912800 & -1.55319900 \\
\hline C & -1.98373300 & 6.08677800 & -1.56908800 \\
\hline C & -3.74389200 & 2.77527400 & -1.81137500 \\
\hline C & -4.21637000 & 4.45555000 & -0.16501300 \\
\hline C & 0.72218500 & 4.38883600 & -2.66419900 \\
\hline C & 1.12815200 & 6.12860000 & -1.05343900 \\
\hline C & -2.49854900 & 5.89906500 & -2.85368700 \\
\hline C & -2.29976700 & 7.25449000 & -0.87861400 \\
\hline $\mathrm{H}$ & -3.04089000 & 2.13516700 & -2.34186200 \\
\hline C & -5.09475700 & 2.72034200 & -2.13858900 \\
\hline C & -5.56354300 & 4.41068900 & -0.50273800 \\
\hline $\mathrm{H}$ & -3.89515300 & 5.15006200 & 0.60703200 \\
\hline $\mathrm{H}$ & 0.09487400 & 3.59712500 & -3.06190600 \\
\hline C & 1.95388300 & 4.66032100 & -3.25521500 \\
\hline C & 2.34103600 & 6.42188000 & -1.66241000 \\
\hline $\mathrm{H}$ & 0.79529900 & 6.70863700 & -0.19242100 \\
\hline $\mathrm{H}$ & -2.24945400 & 4.98615300 & -3.39499400 \\
\hline C & -3.34299800 & 6.84363000 & -3.42023500 \\
\hline C & -3.13931100 & 8.20816400 & -1.44772700 \\
\hline $\mathrm{H}$ & -1.89346300 & 7.41439700 & 0.12014700 \\
\hline $\mathrm{H}$ & -5.42682000 & 2.02882000 & -2.91093800 \\
\hline C & -6.01142500 & 3.53966300 & -1.49030800 \\
\hline $\mathrm{H}$ & -6.26600600 & 5.05812200 & 0.01667300 \\
\hline $\mathrm{H}$ & 2.28299000 & 4.06013400 & -4.10099700 \\
\hline C & 2.75545400 & 5.68808900 & -2.77198000 \\
\hline $\mathrm{H}$ & 2.96462300 & 7.22276500 & -1.27030900 \\
\hline $\mathrm{H}$ & -3.75240300 & 6.67589800 & -4.41376900 \\
\hline C & -3.66949800 & 8.00055800 & -2.71563200 \\
\hline $\mathrm{H}$ & -3.38259000 & 9.11313100 & -0.89516600 \\
\hline $\mathrm{H}$ & 3.70628200 & 5.91306800 & -3.25283400 \\
\hline $\mathrm{H}$ & -4.33422600 & 8.73937300 & -3.15743500 \\
\hline C & -3.14702100 & -2.34409200 & -1.29951700 \\
\hline C & -3.46000100 & -3.28190300 & -0.16932200 \\
\hline C & -4.48305600 & -2.25249200 & -0.51595400 \\
\hline C & -3.22148300 & -2.85932800 & -2.70589100 \\
\hline $\mathrm{H}$ & -3.62347100 & -4.32622900 & -0.42478900 \\
\hline $\mathrm{H}$ & -2.90191700 & -3.11353200 & 0.75145400 \\
\hline C & -4.74291700 & -1.16728400 & 0.48169900 \\
\hline C & -5.71166700 & -2.62333000 & -1.30070700 \\
\hline C & -3.80595000 & -2.08276500 & -3.70866100 \\
\hline C & -2.70849400 & -4.11221800 & -3.02944400 \\
\hline C & -4.37974500 & 0.16086700 & 0.27364300 \\
\hline C & -5.48396600 & -1.49429100 & 1.62074600 \\
\hline & & S158 & \\
\hline
\end{tabular}




\begin{tabular}{|c|c|c|c|}
\hline C & -6.53627800 & -1.60022600 & -1.78222200 \\
\hline C & -6.03597200 & -3.94114300 & -1.62256500 \\
\hline $\mathrm{H}$ & -4.19395400 & -1.09686000 & -3.45258700 \\
\hline C & -3.90390900 & -2.56489700 & -5.00550300 \\
\hline C & -2.79485100 & -4.59399200 & -4.33318000 \\
\hline $\mathrm{H}$ & -2.24044800 & -4.72024100 & -2.25605700 \\
\hline $\mathrm{H}$ & -3.82848300 & 0.43983200 & -0.62562800 \\
\hline C & -4.74450300 & 1.14270600 & 1.18961100 \\
\hline C & -5.83327000 & -0.51875700 & 2.54782600 \\
\hline $\mathrm{H}$ & -5.78483000 & -2.53188000 & 1.77212900 \\
\hline $\mathrm{H}$ & -6.30356300 & -0.56559300 & -1.52947000 \\
\hline C & -7.63056300 & -1.88518400 & -2.58764700 \\
\hline C & -7.13239800 & -4.22919000 & -2.42830700 \\
\hline $\mathrm{H}$ & -5.41850400 & -4.75950900 & -1.25917700 \\
\hline $\mathrm{H}$ & -4.37550000 & -1.95749700 & -5.77478000 \\
\hline C & -3.39931900 & -3.82607000 & -5.32093500 \\
\hline $\mathrm{H}$ & -2.38808000 & -5.57559300 & -4.56987400 \\
\hline $\mathrm{H}$ & -4.46817800 & 2.17768700 & 1.00423000 \\
\hline C & -5.46463400 & 0.80597500 & 2.33109700 \\
\hline $\mathrm{H}$ & -6.39594300 & -0.79303900 & 3.43748000 \\
\hline H & -8.25155600 & -1.07287900 & -2.95928500 \\
\hline C & -7.93060400 & -3.20310600 & -2.91993800 \\
\hline $\mathrm{H}$ & -7.36032100 & -5.26409600 & -2.67361200 \\
\hline H & -5.73136400 & 1.57802600 & 3.05017100 \\
\hline $\mathrm{H}$ & -8.78464200 & -3.42854200 & -3.55444200 \\
\hline $\mathrm{H}$ & -7.06641800 & 3.49951600 & -1.75001800 \\
\hline $\mathrm{H}$ & -3.47785100 & -4.20524900 & -6.33759900 \\
\hline $\mathrm{H}$ & -1.80340100 & -7.39597900 & -0.53727600 \\
\hline $\mathrm{H}$ & 6.97975900 & 0.46379300 & -4.69901100 \\
\hline C & 0.15842200 & -0.32157400 & 2.25326000 \\
\hline C & 0.81448700 & -1.56454500 & 2.71365100 \\
\hline $\mathrm{O}$ & 0.13378600 & -2.68186800 & 2.50945500 \\
\hline C & 0.80466500 & -3.87073800 & 2.93877000 \\
\hline H & 0.95376000 & -3.84542300 & 4.02206400 \\
\hline $\mathrm{H}$ & 1.77420600 & -3.96171900 & 2.43945300 \\
\hline $\mathrm{H}$ & 0.14630900 & -4.69708100 & 2.66692200 \\
\hline $\mathrm{O}$ & 1.93963000 & -1.51510100 & 3.17354100 \\
\hline $\mathrm{O}$ & -0.78841300 & -0.10838200 & 5.43066500 \\
\hline $\mathrm{N}$ & -1.83464600 & -0.53730200 & 4.60390700 \\
\hline C & -1.95254200 & -1.92744800 & 4.48905000 \\
\hline C & -2.33061900 & -4.70248000 & 4.36508800 \\
\hline C & -1.29214900 & -2.76947700 & 5.39186500 \\
\hline C & -2.82404400 & -2.48014800 & 3.54230500 \\
\hline C & -1.48960300 & -4.14337600 & 5.32352200 \\
\hline
\end{tabular}




$\begin{array}{lrrl}\mathrm{C} & -2.99461600 & -3.85598300 & 3.47987900 \\ \mathrm{H} & -0.63747800 & -2.33357500 & 6.13947500 \\ \mathrm{H} & -3.37572100 & -1.84098200 & 2.85825500 \\ \mathrm{H} & -0.97144400 & -4.78554100 & 6.03351500 \\ \mathrm{H} & -3.67376600 & -4.26722700 & 2.73312400 \\ \mathrm{C} & 0.18690500 & 0.63140700 & 4.64530100 \\ \mathrm{H} & 1.13853600 & 0.08562600 & 4.68990100 \\ \mathrm{H} & -2.48108800 & -5.77831800 & 4.31978400 \\ \mathrm{C} & -0.36348900 & 0.66693000 & 3.21415600 \\ \mathrm{H} & -0.27111800 & 1.66425100 & 2.76349000 \\ \mathrm{C} & 0.34357700 & 2.00807600 & 5.24748400 \\ \mathrm{H} & 1.10388500 & 2.56929300 & 4.69277800 \\ \mathrm{H} & -0.60181500 & 2.56025800 & 5.22527300 \\ \mathrm{H} & 0.67172600 & 1.92545200 & 6.28911500 \\ \mathrm{C} & -2.64887700 & 1.64586700 & 3.88895000 \\ \mathrm{C} & -3.38270800 & 1.68435600 & 5.07456600 \\ \mathrm{C} & -2.63897300 & 2.76790300 & 3.06062400 \\ \mathrm{C} & -4.08783800 & 2.82873200 & 5.43248500 \\ \mathrm{H} & -3.38814400 & 0.80949400 & 5.71876000 \\ \mathrm{C} & -3.34525600 & 3.90996000 & 3.42148900 \\ \mathrm{H} & -2.09021600 & 2.73899000 & 2.11990900 \\ \mathrm{C} & -4.07065600 & 3.94845700 & 4.60839800 \\ \mathrm{H} & -4.64929100 & 2.84348600 & 6.36397500 \\ \mathrm{H} & -3.33280600 & 4.77836500 & 2.76509800 \\ \mathrm{H} & -4.61799500 & 4.84582000 & 4.88762000 \\ \mathrm{C} & -1.90281000 & 0.38055800 & 3.50071800 \\ \mathrm{H} & -2.37856300 & -0.08837600 & 2.62654000\end{array}$

s-cis r-4 (-4598.122808 a.u)

$\begin{array}{lccc}\text { C } & 1.79496000 & -1.98388200 & -0.10796600 \\ \text { C } & 2.02013000 & 1.67838700 & -0.38578700 \\ \text { C } & -1.49495800 & 1.96699500 & -1.00294500 \\ \text { C } & -1.93310200 & -1.73475400 & -0.64343100 \\ \text { O } & 1.25420600 & 1.59489200 & 0.63104900 \\ \text { O } & 1.88952400 & 1.03495900 & -1.46436500 \\ \text { O } & -1.52992000 & 1.50249500 & 0.18208200 \\ \text { O } & -0.93491000 & 1.44391500 & -2.00230300 \\ \text { O } & -1.44491400 & -1.47709000 & 0.50647300 \\ \text { O } & -1.44693500 & -1.37521400 & -1.74732600 \\ \text { O } & 1.32247700 & -1.39334700 & 0.91433500 \\ \text { O } & 1.49249400 & -1.76260100 & -1.30787700 \\ \text { Rh } & -0.11981900 & 0.06121900 & 0.67620500 \\ \text { Rh } & 0.24957800 & -0.18569000 & -1.73576400 \\ \text { C } & -2.05572100 & 3.34222400 & -1.25613000 \\ & & & \\ & & S 160 & \end{array}$




\begin{tabular}{|c|c|c|c|}
\hline $\mathrm{C}$ & -2.93214600 & 3.48228700 & -2.45953700 \\
\hline $\mathrm{C}$ & -3.52917700 & 3.69229500 & -1.09855500 \\
\hline C & -1.00521800 & 4.37020400 & -0.94555100 \\
\hline $\mathrm{H}$ & -2.79917800 & 4.34801300 & -3.10439900 \\
\hline $\mathrm{H}$ & -3.16734700 & 2.55654700 & -2.98268700 \\
\hline C & -4.55361000 & 2.72309000 & -0.58720300 \\
\hline C & -3.87410300 & 5.10711300 & -0.71238800 \\
\hline C & -0.56025500 & 4.53150600 & 0.37187600 \\
\hline C & -0.46069800 & 5.17419200 & -1.94607100 \\
\hline C & -4.61739200 & 2.39539700 & 0.76990600 \\
\hline C & -5.57544100 & 2.29093700 & -1.43587300 \\
\hline C & -3.64260200 & 5.57907200 & 0.58232600 \\
\hline $\mathrm{C}$ & -4.50282700 & 5.94839000 & -1.62901900 \\
\hline $\mathrm{H}$ & -0.95633900 & 3.87810900 & 1.15122100 \\
\hline C & 0.36415300 & 5.52164100 & 0.68022400 \\
\hline $\mathrm{C}$ & 0.47349200 & 6.15861400 & -1.63587700 \\
\hline $\mathrm{H}$ & -0.77037500 & 5.02893200 & -2.97842000 \\
\hline $\mathrm{H}$ & -3.79952100 & 2.68518300 & 1.43119600 \\
\hline C & -5.71113400 & 1.69878100 & 1.27064700 \\
\hline C & -6.65642800 & 1.56823800 & -0.93929600 \\
\hline $\mathrm{H}$ & -5.53259700 & 2.55333100 & -2.49293600 \\
\hline $\mathrm{H}$ & -3.16277500 & 4.92879500 & 1.31287200 \\
\hline C & -4.01054900 & 6.86929000 & 0.94097300 \\
\hline C & -4.87808600 & 7.23958600 & -1.27142600 \\
\hline $\mathrm{H}$ & -4.70860800 & 5.58067500 & -2.63402500 \\
\hline $\mathrm{H}$ & 0.68735900 & 5.66109500 & 1.71157200 \\
\hline C & 0.88026200 & 6.34347400 & -0.31915800 \\
\hline $\mathrm{H}$ & 0.88318400 & 6.78175000 & -2.42856000 \\
\hline $\mathrm{H}$ & -5.75707700 & 1.45402700 & 2.32972500 \\
\hline C & -6.73772600 & 1.29316000 & 0.42176600 \\
\hline $\mathrm{H}$ & -7.44092700 & 1.23160900 & -1.61520000 \\
\hline $\mathrm{H}$ & -3.81351900 & 7.22425700 & 1.95031300 \\
\hline C & -4.62791600 & 7.70546900 & 0.01420700 \\
\hline $\mathrm{H}$ & -5.36838200 & 7.88168500 & -2.00029700 \\
\hline $\mathrm{H}$ & -7.58816100 & 0.74488200 & 0.82278100 \\
\hline $\mathrm{H}$ & -4.91542000 & 8.71587900 & 0.29591900 \\
\hline C & -3.17204600 & -2.57420000 & -0.66757200 \\
\hline C & -3.04280500 & -3.86721700 & 0.11187400 \\
\hline C & -3.97803200 & -2.83590800 & 0.62760300 \\
\hline C & -3.95285800 & -2.46980600 & -1.93834700 \\
\hline $\mathrm{H}$ & -3.44995700 & -4.77044000 & -0.33509000 \\
\hline $\mathrm{H}$ & -2.09579900 & -4.00809500 & 0.63232200 \\
\hline C & -3.72121400 & -2.14363900 & 1.93385600 \\
\hline $\mathrm{C}$ & -5.44701900 & -3.10620300 & 0.42917300 \\
\hline
\end{tabular}




\begin{tabular}{|c|c|c|c|}
\hline C & -4.37053200 & -1.19663700 & -2.34517400 \\
\hline C & -4.35808200 & -3.57488700 & -2.67836100 \\
\hline C & -3.72096400 & -0.75471600 & 2.04845200 \\
\hline C & -3.60379100 & -2.91331900 & 3.09171900 \\
\hline C & -6.30995700 & -2.06211500 & 0.09369600 \\
\hline C & -5.97398900 & -4.38486300 & 0.59796800 \\
\hline $\mathrm{H}$ & -4.07185100 & -0.31940600 & -1.76421300 \\
\hline C & -5.18424900 & -1.03814600 & -3.45607300 \\
\hline C & -5.17907400 & -3.41964200 & -3.79330300 \\
\hline $\mathrm{H}$ & -4.04415500 & -4.57318900 & -2.37577200 \\
\hline $\mathrm{H}$ & -3.77557000 & -0.13802400 & 1.15140700 \\
\hline C & -3.61832500 & -0.14663300 & 3.29484300 \\
\hline C & -3.51181800 & -2.30824600 & 4.34417000 \\
\hline $\mathrm{H}$ & -3.60997400 & -4.00097400 & 3.00616900 \\
\hline $\mathrm{H}$ & -5.91018500 & -1.05674300 & -0.02700600 \\
\hline C & -7.66146300 & -2.29994800 & -0.11478000 \\
\hline C & -7.33248500 & -4.62399800 & 0.40846500 \\
\hline $\mathrm{H}$ & -5.31331000 & -5.20507000 & 0.87841700 \\
\hline $\mathrm{H}$ & -5.50516300 & -0.03959500 & -3.74830300 \\
\hline C & -5.59656700 & -2.15334600 & -4.18351700 \\
\hline H & -5.49433400 & -4.29568900 & -4.35560500 \\
\hline $\mathrm{H}$ & -3.58003800 & 0.93936200 & 3.35670600 \\
\hline C & -3.52519800 & -0.92188800 & 4.44883900 \\
\hline H & -3.43640100 & -2.92542100 & 5.23809500 \\
\hline $\mathrm{H}$ & -8.31386100 & -1.47785300 & -0.40572400 \\
\hline C & -8.17834000 & -3.58399900 & 0.03987600 \\
\hline $\mathrm{H}$ & -7.72814100 & -5.62843900 & 0.54258500 \\
\hline $\mathrm{H}$ & -3.45163300 & -0.44400700 & 5.42371000 \\
\hline $\mathrm{H}$ & -9.23692600 & -3.77189100 & -0.12466700 \\
\hline C & 2.87964700 & -2.99846000 & 0.14607800 \\
\hline C & 2.73084200 & -3.87971200 & 1.34680400 \\
\hline C & 2.53080200 & -4.48077800 & -0.01257200 \\
\hline C & 4.23461400 & -2.49380500 & -0.27639800 \\
\hline $\mathrm{H}$ & 3.61900700 & -4.15000700 & 1.91297800 \\
\hline $\mathrm{H}$ & 1.83827000 & -3.71759800 & 1.94864900 \\
\hline C & 1.14960000 & -4.94299200 & -0.39180500 \\
\hline C & 3.60409500 & -5.37794300 & -0.56096800 \\
\hline C & 4.42196000 & -1.96557200 & -1.55968100 \\
\hline C & 5.32616400 & -2.52323100 & 0.59407700 \\
\hline C & 0.52929000 & -4.56321600 & -1.58564800 \\
\hline C & 0.53472100 & -5.91311400 & 0.40679800 \\
\hline C & 3.89386200 & -5.35372500 & -1.92704100 \\
\hline C & 4.29352200 & -6.26979500 & 0.25782400 \\
\hline $\mathrm{H}$ & 3.57545400 & -1.90437100 & -2.23972700 \\
\hline
\end{tabular}




\begin{tabular}{|c|c|c|c|}
\hline C & 5.67019600 & -1.50590600 & -1.96225100 \\
\hline C & 6.57464000 & -2.05765500 & 0.19280400 \\
\hline $\mathrm{H}$ & 5.20444200 & -2.89720800 & 1.60847300 \\
\hline $\mathrm{H}$ & 0.98248700 & -3.79514500 & -2.20624300 \\
\hline C & -0.66901000 & -5.15241100 & -1.97697900 \\
\hline C & -0.65110200 & -6.51865400 & 0.00597100 \\
\hline $\mathrm{H}$ & 1.02041000 & -6.21779900 & 1.33461700 \\
\hline $\mathrm{H}$ & 3.35299800 & -4.66217700 & -2.57235400 \\
\hline C & 4.86390600 & -6.19209700 & -2.45885700 \\
\hline C & 5.26404000 & -7.11498100 & -0.27323800 \\
\hline $\mathrm{H}$ & 4.06417200 & -6.30733300 & 1.32218300 \\
\hline $\mathrm{H}$ & 5.79161900 & -1.10634500 & -2.96712700 \\
\hline C & 6.75327200 & -1.55119000 & -1.08988100 \\
\hline $\mathrm{H}$ & 7.40796300 & -2.08507900 & 0.89220400 \\
\hline $\mathrm{H}$ & -1.14554000 & -4.83103700 & -2.90116300 \\
\hline C & -1.24851700 & -6.14701500 & -1.19562100 \\
\hline $\mathrm{H}$ & -1.10444600 & -7.28780100 & 0.62809600 \\
\hline $\mathrm{H}$ & 5.08519300 & -6.15511100 & -3.52320100 \\
\hline C & 5.55386000 & -7.07533500 & -1.63204500 \\
\hline $\mathrm{H}$ & 5.79434100 & -7.80576900 & 0.37848900 \\
\hline $\mathrm{H}$ & -2.17285200 & -6.62504800 & -1.51651000 \\
\hline $\mathrm{H}$ & 6.31507900 & -7.73142800 & -2.04777400 \\
\hline C & 3.18016500 & 2.62114300 & -0.29737200 \\
\hline C & 3.14927200 & 3.69447900 & 0.75688700 \\
\hline C & 4.11676100 & 2.57905200 & 0.95013500 \\
\hline C & 3.82506700 & 2.93277200 & -1.61215500 \\
\hline $\mathrm{H}$ & 3.49453700 & 4.68191200 & 0.46006900 \\
\hline $\mathrm{H}$ & 2.27797600 & 3.68963600 & 1.40825400 \\
\hline C & 3.86056400 & 1.65120400 & 2.10086700 \\
\hline C & 5.58001300 & 2.76722300 & 0.65972800 \\
\hline C & 4.67126300 & 1.99830000 & -2.21092600 \\
\hline C & 3.60791700 & 4.15387100 & -2.24387000 \\
\hline C & 3.63532100 & 0.28909100 & 1.93755100 \\
\hline C & 3.92421400 & 2.17107700 & 3.39569100 \\
\hline C & 6.40583700 & 1.63922200 & 0.60182300 \\
\hline C & 6.14394100 & 4.01403200 & 0.38940000 \\
\hline $\mathrm{H}$ & 4.83021300 & 1.03856300 & -1.71895200 \\
\hline C & 5.31827900 & 2.29454800 & -3.40144400 \\
\hline C & 4.24586600 & 4.44944300 & -3.44631900 \\
\hline $\mathrm{H}$ & 2.93005000 & 4.87700600 & -1.78906000 \\
\hline $\mathrm{H}$ & 3.60824100 & -0.12943100 & 0.93150800 \\
\hline C & 3.43147500 & -0.53403500 & 3.04112100 \\
\hline C & 3.73402600 & 1.35245900 & 4.50177800 \\
\hline $\mathrm{H}$ & 4.13258600 & 3.23446200 & 3.52394100 \\
\hline
\end{tabular}




\begin{tabular}{|c|c|c|c|}
\hline $\mathrm{H}$ & 5.98768000 & 0.65243300 & 0.80391700 \\
\hline C & 7.74574800 & 1.75292300 & 0.25814800 \\
\hline C & 7.48710100 & 4.13039200 & 0.04557400 \\
\hline $\mathrm{H}$ & 5.52859800 & 4.91034500 & 0.43054600 \\
\hline $\mathrm{H}$ & 5.99386000 & 1.56652700 & -3.84671700 \\
\hline C & 5.10955700 & 3.52538000 & -4.02175500 \\
\hline $\mathrm{H}$ & 4.06910700 & 5.40807100 & -3.93021400 \\
\hline $\mathrm{H}$ & 3.21529800 & -1.59029500 & 2.88410200 \\
\hline C & 3.47526300 & -0.00486100 & 4.32543100 \\
\hline $\mathrm{H}$ & 3.78212200 & 1.77446200 & 5.50408100 \\
\hline $\mathrm{H}$ & 8.36380400 & 0.85755300 & 0.21121400 \\
\hline C & 8.29351300 & 3.00053500 & -0.02610800 \\
\hline $\mathrm{H}$ & 7.90256700 & 5.11266100 & -0.16894500 \\
\hline $\mathrm{H}$ & 3.30887200 & -0.64774600 & 5.18808100 \\
\hline $\mathrm{H}$ & 9.34243800 & 3.09159600 & -0.29902300 \\
\hline $\mathrm{H}$ & 7.72781400 & -1.18690000 & -1.40766100 \\
\hline $\mathrm{H}$ & 5.61992400 & 3.76061400 & -4.95335600 \\
\hline $\mathrm{H}$ & 1.60108000 & 7.12016100 & -0.07116600 \\
\hline $\mathrm{H}$ & -6.24184600 & -2.03329000 & -5.05084700 \\
\hline C & -0.37082300 & 0.24908800 & 2.63306100 \\
\hline C & -0.48527700 & 1.64262700 & 3.12177900 \\
\hline $\mathrm{O}$ & 0.68097100 & 2.06198400 & 3.61892000 \\
\hline C & 0.72726400 & 3.43404100 & 3.99624300 \\
\hline $\mathrm{H}$ & 0.04833400 & 3.63028900 & 4.83232100 \\
\hline $\mathrm{H}$ & 0.43987900 & 4.07018800 & 3.15081600 \\
\hline $\mathrm{H}$ & 1.76251900 & 3.62707400 & 4.28447800 \\
\hline $\mathrm{O}$ & -1.48990500 & 2.32014100 & 3.05889900 \\
\hline C & -0.27250700 & -2.09880300 & 3.28199700 \\
\hline $\mathrm{H}$ & -0.33212500 & -2.37976700 & 2.22980600 \\
\hline C & -0.32415400 & -0.77449600 & 3.60450400 \\
\hline $\mathrm{H}$ & -0.30699000 & -0.49995400 & 4.66562300 \\
\hline C & -0.11092400 & -3.17921100 & 4.27614300 \\
\hline $\mathrm{H}$ & 0.89754000 & -3.61095800 & 4.18083000 \\
\hline $\mathrm{H}$ & -0.23646900 & -2.82449300 & 5.30473600 \\
\hline $\mathrm{H}$ & -0.81456900 & -3.99819000 & 4.08259200 \\
\hline
\end{tabular}

$\begin{array}{cccc}\text { r-TS(cx)-I (-5229.567197 a.u.) } & & \\ \text { C } & 1.61878500 & -2.12151300 & -0.72463100 \\ \text { C } & 1.90426700 & 1.55473700 & -1.30291800 \\ \text { C } & -1.59832700 & 1.92146000 & -1.49818100 \\ \text { C } & -2.16000900 & -1.71676400 & -0.87245000 \\ \text { O } & 1.46071000 & 1.37413300 & -0.12480700 \\ \text { O } & 1.47557700 & 1.02358000 & -2.36307800 \\ \text { O } & -1.35008300 & 1.64362300 & -0.28185700 \\ & & & \end{array}$




\begin{tabular}{|c|c|c|c|}
\hline $\mathrm{O}$ & -1.37971000 & 1.19198300 & -2.50062200 \\
\hline $\mathrm{O}$ & -1.66563300 & -1.31288900 & 0.22770800 \\
\hline $\mathrm{O}$ & -1.63133200 & -1.60793000 & -2.01099900 \\
\hline $\mathrm{O}$ & 1.21243700 & -1.56301800 & 0.34323200 \\
\hline $\mathrm{O}$ & 1.35615800 & -1.79506500 & -1.90840700 \\
\hline $\mathrm{Rh}$ & -0.09069300 & 0.04463100 & 0.15330700 \\
\hline $\mathrm{Rh}$ & -0.04465900 & -0.34234400 & -2.28866100 \\
\hline C & -2.08810500 & 3.31479200 & -1.80337100 \\
\hline C & -2.93639800 & 3.48768200 & -3.01959000 \\
\hline C & -3.55047400 & 3.76305100 & -1.67731900 \\
\hline C & -1.04250700 & 4.34824100 & -1.50178900 \\
\hline $\mathrm{H}$ & -2.71092500 & 4.30391900 & -3.70000200 \\
\hline $\mathrm{H}$ & -3.24483900 & 2.56614800 & -3.50987300 \\
\hline C & -4.62916000 & 2.83848800 & -1.20292400 \\
\hline C & -3.76157300 & 5.19053400 & -1.23256300 \\
\hline C & -0.89491800 & 4.83035200 & -0.19881200 \\
\hline C & -0.28181500 & 4.91208500 & -2.52545900 \\
\hline C & -4.60860100 & 2.25249100 & 0.06579500 \\
\hline C & -5.77358200 & 2.70539900 & -1.99496300 \\
\hline C & -4.10439400 & 5.45741900 & 0.09777700 \\
\hline C & -3.55474700 & 6.27732700 & -2.08392000 \\
\hline $\mathrm{H}$ & -1.46772700 & 4.36093600 & 0.60212200 \\
\hline C & -0.08095900 & 5.92884100 & 0.05218600 \\
\hline $\mathrm{C}$ & 0.54909400 & 6.00006200 & -2.27019500 \\
\hline $\mathrm{H}$ & -0.36782300 & 4.51046900 & -3.53429100 \\
\hline $\mathrm{H}$ & -3.70580900 & 2.31634100 & 0.67929300 \\
\hline $\mathrm{C}$ & -5.74847700 & 1.61709700 & 0.55367100 \\
\hline C & -6.89796900 & 2.04201500 & -1.51928000 \\
\hline $\mathrm{H}$ & -5.78036300 & 3.15858000 & -2.98615300 \\
\hline $\mathrm{H}$ & -4.28098400 & 4.62967200 & 0.78202100 \\
\hline C & -4.20155300 & 6.76102200 & 0.56662000 \\
\hline C & -3.64991500 & 7.58426200 & -1.61752400 \\
\hline $\mathrm{H}$ & -3.30161000 & 6.11211100 & -3.12826700 \\
\hline $\mathrm{H}$ & -0.01956300 & 6.33614200 & 1.06015000 \\
\hline $\mathrm{C}$ & 0.62907000 & 6.52833400 & -0.98623400 \\
\hline $\mathrm{H}$ & 1.12115000 & 6.44542000 & -3.08206100 \\
\hline $\mathrm{H}$ & -5.73646800 & 1.18015600 & 1.54971200 \\
\hline C & -6.89594800 & 1.52469500 & -0.22759700 \\
\hline $\mathrm{H}$ & -7.78161500 & 1.94751000 & -2.14728700 \\
\hline $\mathrm{H}$ & -4.46006400 & 6.93756100 & 1.60865600 \\
\hline C & -3.96694800 & 7.83375500 & -0.28775300 \\
\hline $\mathrm{H}$ & -3.47481600 & 8.41092200 & -2.30274000 \\
\hline $\mathrm{H}$ & -7.78024400 & 1.02872400 & 0.16847300 \\
\hline $\mathrm{H}$ & -4.03736500 & 8.85549800 & 0.07856400 \\
\hline
\end{tabular}




\begin{tabular}{|c|c|c|c|}
\hline C & -3.47342900 & -2.45562500 & -0.87826100 \\
\hline C & -3.42784400 & -3.76299300 & -0.11854400 \\
\hline C & -4.37990800 & -2.71083300 & 0.34598200 \\
\hline C & -4.19316200 & -2.28971000 & -2.18368700 \\
\hline $\mathrm{H}$ & -3.81558800 & -4.66034200 & -0.59443500 \\
\hline $\mathrm{H}$ & -2.51379100 & -3.92336200 & 0.45344200 \\
\hline C & -4.28241500 & -2.05473500 & 1.69520100 \\
\hline C & -5.82805600 & -2.94924500 & -0.01244800 \\
\hline C & -4.50379300 & -0.98407000 & -2.58269100 \\
\hline C & -4.64655100 & -3.35157700 & -2.95730900 \\
\hline C & -3.91576000 & -0.72473300 & 1.88880600 \\
\hline C & -4.71962800 & -2.79558600 & 2.80083200 \\
\hline C & -6.62856400 & -1.85989400 & -0.35752000 \\
\hline C & -6.39512600 & -4.22177400 & -0.00874000 \\
\hline $\mathrm{H}$ & -4.15351400 & -0.13833500 & -1.98307400 \\
\hline C & -5.27133100 & -0.75055300 & -3.71293200 \\
\hline C & -5.42199100 & -3.12119300 & -4.09188800 \\
\hline $\mathrm{H}$ & -4.41330000 & -4.37404400 & -2.66181000 \\
\hline $\mathrm{H}$ & -3.56577000 & -0.12973700 & 1.04815700 \\
\hline C & -3.97356500 & -0.14935600 & 3.15767600 \\
\hline C & -4.78288800 & -2.22620500 & 4.06596800 \\
\hline $\mathrm{H}$ & -5.02635500 & -3.83125100 & 2.65213400 \\
\hline $\mathrm{H}$ & -6.19129200 & -0.86338600 & -0.35377500 \\
\hline $\mathrm{C}$ & -7.94692200 & -2.04135700 & -0.74752900 \\
\hline C & -7.72547900 & -4.40680300 & -0.37798600 \\
\hline $\mathrm{H}$ & -5.78932600 & -5.08233500 & 0.27401000 \\
\hline $\mathrm{H}$ & -5.51324400 & 0.27357400 & -3.99276700 \\
\hline $\mathrm{C}$ & -5.74104400 & -1.82286900 & -4.47013900 \\
\hline $\mathrm{H}$ & -5.78024100 & -3.96437500 & -4.67866400 \\
\hline $\mathrm{H}$ & -3.64829900 & 0.88434200 & 3.27797000 \\
\hline $\mathrm{C}$ & -4.40956400 & -0.89526600 & 4.24730800 \\
\hline $\mathrm{H}$ & -5.13106600 & -2.81939600 & 4.90935300 \\
\hline $\mathrm{H}$ & -8.54040300 & -1.18097900 & -1.05355700 \\
\hline C & -8.50113000 & -3.31974100 & -0.76263700 \\
\hline $\mathrm{H}$ & -8.15182100 & -5.40785500 & -0.37447300 \\
\hline $\mathrm{H}$ & -4.45979200 & -0.43911900 & 5.23470900 \\
\hline $\mathrm{H}$ & -9.53362000 & -3.46639300 & -1.07194500 \\
\hline C & 2.61476800 & -3.24306100 & -0.56460300 \\
\hline C & 2.43626700 & -4.20008000 & 0.56320700 \\
\hline $\mathrm{C}$ & 2.20092200 & -4.69041200 & -0.84365100 \\
\hline $\mathrm{C}$ & 3.96079700 & -2.71081600 & -0.95481600 \\
\hline $\mathrm{H}$ & 3.30682200 & -4.55958000 & 1.10645200 \\
\hline $\mathrm{H}$ & 1.53735100 & -4.07703100 & 1.16739700 \\
\hline $\mathrm{C}$ & 0.77540800 & -5.02825600 & -1.18564300 \\
\hline
\end{tabular}




\begin{tabular}{|c|c|c|c|}
\hline C & 3.22209800 & -5.59916600 & -1.47642600 \\
\hline C & 4.41223500 & -2.77163500 & -2.27497800 \\
\hline C & 4.71864600 & -2.02128500 & -0.00878700 \\
\hline C & 0.10471600 & -4.47707000 & -2.27935400 \\
\hline C & 0.14820400 & -6.03923600 & -0.44932800 \\
\hline C & 2.91607100 & -6.27543900 & -2.66238600 \\
\hline C & 4.50362500 & -5.77290000 & -0.94107000 \\
\hline $\mathrm{H}$ & 3.81004900 & -3.29142700 & -3.01827800 \\
\hline C & 5.61594500 & -2.17501000 & -2.62974000 \\
\hline C & 5.91998500 & -1.41290000 & -0.36453100 \\
\hline $\mathrm{H}$ & 4.34151300 & -1.94257100 & 1.01094400 \\
\hline $\mathrm{H}$ & 0.57866100 & -3.68585600 & -2.85518900 \\
\hline C & -1.16671300 & -4.92775700 & -2.62716200 \\
\hline C & -1.11113600 & -6.50432000 & -0.80649900 \\
\hline $\mathrm{H}$ & 0.67624000 & -6.48009400 & 0.39629300 \\
\hline $\mathrm{H}$ & 1.92547300 & -6.16874900 & -3.09853400 \\
\hline C & 3.85529000 & -7.08446900 & -3.29240900 \\
\hline C & 5.44284700 & -6.58179900 & -1.56809200 \\
\hline $\mathrm{H}$ & 4.79619700 & -5.25299800 & -0.03093700 \\
\hline $\mathrm{H}$ & 5.96452900 & -2.23871800 & -3.65843100 \\
\hline C & 6.37073600 & -1.48942800 & -1.67863900 \\
\hline $\mathrm{H}$ & 6.48992400 & -0.86501300 & 0.38568600 \\
\hline $\mathrm{H}$ & -1.68790000 & -4.46520600 & -3.46303400 \\
\hline C & -1.76793500 & -5.95171800 & -1.90386000 \\
\hline $\mathrm{H}$ & -1.57847100 & -7.30174700 & -0.23241800 \\
\hline $\mathrm{H}$ & 3.58681200 & -7.59518600 & -4.21460500 \\
\hline C & 5.12490100 & -7.24270900 & -2.74957700 \\
\hline $\mathrm{H}$ & 6.43168500 & -6.69211900 & -1.12833300 \\
\hline $\mathrm{H}$ & -2.75307500 & -6.31708700 & -2.19123100 \\
\hline $\mathrm{H}$ & 5.86029300 & -7.87505600 & -3.24150500 \\
\hline C & 3.01683400 & 2.54413800 & -1.49322100 \\
\hline C & 2.76739500 & 3.89740600 & -0.86213800 \\
\hline C & 3.85788600 & 3.05363500 & -0.31087300 \\
\hline C & 3.71185700 & 2.42709900 & -2.81415400 \\
\hline $\mathrm{H}$ & 3.02173300 & 4.78036200 & -1.44231600 \\
\hline $\mathrm{H}$ & 1.84671300 & 3.98000600 & -0.28432900 \\
\hline C & 3.77609900 & 2.52001400 & 1.08539100 \\
\hline C & 5.28070800 & 3.43078700 & -0.63588700 \\
\hline C & 4.30414500 & 1.20363700 & -3.14565100 \\
\hline C & 3.84384300 & 3.49370700 & -3.69690400 \\
\hline C & 3.99386100 & 1.17303800 & 1.36914900 \\
\hline C & 3.67973000 & 3.42762000 & 2.13923700 \\
\hline C & 6.23458500 & 2.43060300 & -0.84252600 \\
\hline C & 5.68478000 & 4.76268000 & -0.69147600 \\
\hline
\end{tabular}




$\begin{array}{ccc}4.18892600 & 0.35091200 & -2.47256900 \\ 5.03262600 & 1.06232400 & -4.31603800 \\ 4.57360200 & 3.35461900 & -4.87570700 \\ 3.37309300 & 4.44824400 & -3.46623700 \\ 4.04553000 & 0.45580800 & 0.55052800 \\ 4.16907600 & 0.75102000 & 2.67839000 \\ 3.83476300 & 3.00767800 & 3.45655000 \\ 3.51532300 & 4.48190000 & 1.91228000 \\ 5.92915000 & 1.38507900 & -0.80083700 \\ 7.55297000 & 2.75565000 & -1.12702400 \\ 7.00931100 & 5.09270000 & -0.96723700 \\ 4.95267800 & 5.55085400 & -0.51789700 \\ 5.49142400 & 0.10292300 & -4.54873400 \\ 5.17595300 & 2.14223200 & -5.18577600 \\ 4.67027300 & 4.20138600 & -5.55191500 \\ 4.38217600 & -0.29726800 & 2.87912300 \\ 4.10612800 & 1.67008600 & 3.72601300 \\ 3.78247400 & 3.73161700 & 4.26853800 \\ 8.27692800 & 1.96212200 & -1.30351400 \\ 7.94592700 & 4.09074700 & -1.19162600 \\ 7.30759700 & 6.13816100 & -1.00946100 \\ 4.30489900 & 1.34656400 & 4.74841600 \\ 8.97880400 & 4.34744100 & -1.41619900 \\ 7.30098500 & -1.00289700 & -1.96726800 \\ 5.75130800 & 2.03490900 & -6.10272700 \\ 1.25123700 & 7.39922100 & -0.79059100 \\ -6.35281700 & -1.64467200 & -5.35174000 \\ -0.39108800 & 0.39177700 & 2.13953900 \\ -0.69675200 & 1.80359100 & 2.45614400 \\ 0.45957300 & 2.48223400 & 2.60540600 \\ 0.38491000 & 3.87536600 & 2.85856800 \\ 0.90607200 & 4.39498000 & 2.04803200 \\ 0.89805600 & 4.08850700 & 3.80443400 \\ -0.65704300 & 4.20588400 & 2.91470300 \\ -1.80064800 & 2.29705800 & 2.57165900 \\ 0.69591600 & -2.20915200 & 4.54270200 \\ 1.65486800 & -1.33028100 & 4.73156800 \\ 2.99089800 & -1.80905800 & 4.52414500 \\ 5.56377700 & -2.75893800 & 4.16397500 \\ 3.24187400 & -2.70492500 & 3.49271200 \\ 3.99690600 & -1.41489300 & 5.40336700 \\ 4.53758000 & -3.17503000 & 3.31882300 \\ 5.29116600 & -1.88134700 & 5.20845200 \\ 2.43286200 & -3.00201300 & 2.83199400\end{array}$




$\begin{array}{lccc}\text { H } & 3.76168200 & -0.77082500 & 6.24779800 \\ \mathrm{H} & 4.75574100 & -3.86137400 & 2.50315000 \\ \mathrm{H} & 6.08162100 & -1.57323800 & 5.88745100 \\ \mathrm{C} & -0.39920800 & -1.91026900 & 3.03869200 \\ \mathrm{H} & 0.30451900 & -2.17855500 & 2.24611200 \\ \mathrm{H} & 6.57381900 & -3.13094100 & 4.01412600 \\ \mathrm{C} & -0.73662600 & -0.52772100 & 3.08799000 \\ \mathrm{H} & -1.37586900 & -0.21394600 & 3.91724900 \\ \mathrm{C} & -1.35839900 & -2.96193000 & 3.45356000 \\ \mathrm{H} & -1.94342700 & -2.65091600 & 4.32606500 \\ \mathrm{H} & -2.06439300 & -3.10533000 & 2.62569800 \\ \mathrm{H} & -0.85433100 & -3.91113200 & 3.65741700 \\ \mathrm{C} & 0.24815600 & 0.43487400 & 5.74519100 \\ \mathrm{C} & -0.73997700 & -0.35552600 & 6.35481100 \\ \mathrm{C} & 0.11200500 & 1.82938400 & 5.72952300 \\ \mathrm{C} & -1.86112000 & 0.25132900 & 6.89415200 \\ \mathrm{H} & -0.63179700 & -1.43640400 & 6.35729700 \\ \mathrm{C} & -1.03278300 & 2.42555600 & 6.23730900 \\ \mathrm{H} & 0.89686600 & 2.42633600 & 5.26483600 \\ \mathrm{C} & -2.02399900 & 1.63608400 & 6.81320200 \\ \mathrm{H} & -2.62899400 & -0.35939900 & 7.36285200 \\ \mathrm{H} & -1.15448700 & 3.50428100 & 6.17802600 \\ \mathrm{H} & -2.92582100 & 2.09895300 & 7.20635400 \\ \mathrm{C} & 1.43767200 & -0.09878000 & 5.13066600 \\ \mathrm{H} & 2.29132100 & 0.56693000 & 5.00439700\end{array}$

$\begin{array}{cccc}\text { r-(cx)-A (-5229.574732 a.u.) } & & \\ \text { C } & 1.66947600 & -1.94934900 & -0.97079900 \\ \text { C } & 1.87097500 & 1.72171400 & -1.15572200 \\ \text { C } & -1.67766800 & 2.04070100 & -1.36330700 \\ \text { C } & -2.13071600 & -1.63289600 & -1.14297700 \\ \text { O } & 1.51553100 & 1.28575500 & -0.01703400 \\ \text { O } & 1.38352800 & 1.41126600 & -2.27658900 \\ \text { O } & -1.29592700 & 1.77193300 & -0.17999900 \\ \text { O } & -1.48872000 & 1.34735400 & -2.39803000 \\ \text { O } & -1.79373300 & -1.15759500 & -0.01577100 \\ \text { O } & -1.49471900 & -1.52448200 & -2.22632900 \\ \text { O } & 1.09745100 & -1.59643600 & 0.10610000 \\ \text { O } & 1.50970300 & -1.46038700 & -2.11680900 \\ \text { Rh } & -0.12540100 & 0.06671100 & 0.09140100 \\ \text { Rh } & -0.02109800 & -0.08609600 & -2.36995600 \\ \text { C } & -2.30031400 & 3.39600800 & -1.61406100 \\ \text { C } & -3.35381400 & 3.46599200 & -2.67070900 \\ \text { C } & -3.73895500 & 3.77127800 & -1.24879400 \\ & & & \\ & & 5169 & \end{array}$




\begin{tabular}{|c|c|c|c|}
\hline C & -1.22784300 & 4.44756200 & -1.59801200 \\
\hline $\mathrm{H}$ & -3.29385100 & 4.26530800 & -3.40488500 \\
\hline $\mathrm{H}$ & -3.68784700 & 2.51089200 & -3.07269500 \\
\hline C & -4.63440200 & 2.78991600 & -0.55494700 \\
\hline C & -3.99213400 & 5.20020300 & -0.83522000 \\
\hline C & -0.97445500 & 5.21738200 & -0.46100200 \\
\hline C & -0.47041700 & 4.66838000 & -2.74935100 \\
\hline C & -4.28852400 & 2.21691500 & 0.66969400 \\
\hline C & -5.91282200 & 2.56338100 & -1.07147800 \\
\hline C & -4.30323700 & 5.49203500 & 0.49847100 \\
\hline C & -3.86572000 & 6.27104000 & -1.72328700 \\
\hline $\mathrm{H}$ & -1.55657900 & 5.03632900 & 0.44028400 \\
\hline C & -0.03834400 & 6.24394400 & -0.49830400 \\
\hline C & 0.47991300 & 5.68445400 & -2.78395900 \\
\hline $\mathrm{H}$ & -0.64485000 & 4.04312000 & -3.62414700 \\
\hline $\mathrm{H}$ & -3.29150400 & 2.36669200 & 1.07824300 \\
\hline C & -5.21925900 & 1.46741900 & 1.38346200 \\
\hline C & -6.83385000 & 1.79001800 & -0.37448100 \\
\hline $\mathrm{H}$ & -6.18528700 & 3.02130900 & -2.02284700 \\
\hline $\mathrm{H}$ & -4.42977000 & 4.67682400 & 1.20868400 \\
\hline C & -4.44399900 & 6.80415100 & 0.93330100 \\
\hline C & -4.00882900 & 7.58491400 & -1.29174500 \\
\hline $\mathrm{H}$ & -3.62728600 & 6.09012800 & -2.76840800 \\
\hline H & 0.12439700 & 6.85837900 & 0.38538100 \\
\hline C & 0.68014800 & 6.48957800 & -1.66628100 \\
\hline $\mathrm{H}$ & 1.05686800 & 5.85212400 & -3.69114500 \\
\hline $\mathrm{H}$ & -4.93499700 & 1.05405400 & 2.34856200 \\
\hline C & -6.49382300 & 1.25581200 & 0.86692000 \\
\hline $\mathrm{H}$ & -7.82263900 & 1.61608800 & -0.79453700 \\
\hline $\mathrm{H}$ & -4.67766100 & 7.00000700 & 1.97786300 \\
\hline C & -4.29103700 & 7.86037100 & 0.04104500 \\
\hline $\mathrm{H}$ & -3.89496100 & 8.39741400 & -2.00609600 \\
\hline $\mathrm{H}$ & -7.21674400 & 0.66299900 & 1.42523800 \\
\hline H & -4.39839800 & 8.88829900 & 0.37994200 \\
\hline C & -3.42200400 & -2.40497400 & -1.26984500 \\
\hline C & -3.32447500 & -3.87209100 & -0.94290700 \\
\hline C & -4.23672700 & -3.01740200 & -0.12292700 \\
\hline C & -4.19285000 & -1.91409100 & -2.45907700 \\
\hline $\mathrm{H}$ & -3.74502200 & -4.59320300 & -1.64044000 \\
\hline $\mathrm{H}$ & -2.38784300 & -4.19241100 & -0.48451600 \\
\hline C & -4.00221900 & -2.84057800 & 1.35131700 \\
\hline C & -5.71579700 & -3.15253900 & -0.39052400 \\
\hline C & -4.62403000 & -0.58386300 & -2.44852200 \\
\hline C & -4.50005000 & -2.71314000 & -3.55429900 \\
\hline
\end{tabular}




\begin{tabular}{|c|c|c|c|}
\hline C & -3.85119800 & -1.59561200 & 1.95918500 \\
\hline C & -4.10559400 & -3.97601800 & 2.16118500 \\
\hline C & -6.53786700 & -2.03307400 & -0.24995700 \\
\hline C & -6.29879500 & -4.37263700 & -0.72549200 \\
\hline $\mathrm{H}$ & -4.38732500 & 0.05521800 & -1.59216500 \\
\hline C & -5.36231500 & -0.07183000 & -3.50552200 \\
\hline C & -5.24070200 & -2.20151100 & -4.61702200 \\
\hline $\mathrm{H}$ & -4.15479100 & -3.74601900 & -3.58016800 \\
\hline $\mathrm{H}$ & -3.74147900 & -0.70600700 & 1.34380600 \\
\hline C & -3.79862000 & -1.48772200 & 3.34666500 \\
\hline C & -4.05918100 & -3.87321300 & 3.54669400 \\
\hline $\mathrm{H}$ & -4.23789400 & -4.94961500 & 1.68825300 \\
\hline $\mathrm{H}$ & -6.08922400 & -1.07803000 & 0.01986100 \\
\hline C & -7.90487700 & -2.12337200 & -0.46747300 \\
\hline C & -7.67233200 & -4.46992900 & -0.93533300 \\
\hline $\mathrm{H}$ & -5.67395900 & -5.25984000 & -0.82255800 \\
\hline $\mathrm{H}$ & -5.70345700 & 0.96205900 & -3.46872200 \\
\hline C & -5.67669600 & -0.88189600 & -4.59473200 \\
\hline $\mathrm{H}$ & -5.47719700 & -2.83964900 & -5.46590200 \\
\hline $\mathrm{H}$ & -3.64211500 & -0.50431800 & 3.79080100 \\
\hline C & -3.90504200 & -2.62338600 & 4.14388900 \\
\hline $\mathrm{H}$ & -4.14578500 & -4.76803800 & 4.16003600 \\
\hline $\mathrm{H}$ & -8.52652600 & -1.23476300 & -0.36948600 \\
\hline C & -8.47889300 & -3.34498600 & -0.81312400 \\
\hline $\mathrm{H}$ & -8.11101300 & -5.43045500 & -1.19781300 \\
\hline $\mathrm{H}$ & -3.86549900 & -2.53498900 & 5.22893000 \\
\hline $\mathrm{H}$ & -9.55050600 & -3.41825400 & -0.98518400 \\
\hline C & 2.72606200 & -3.01954800 & -0.82848300 \\
\hline C & 2.51844500 & -4.07121700 & 0.20725100 \\
\hline C & 2.41574800 & -4.45933300 & -1.24658300 \\
\hline C & 4.07071700 & -2.39561900 & -1.03409500 \\
\hline $\mathrm{H}$ & 3.37542400 & -4.42346600 & 0.77772200 \\
\hline $\mathrm{H}$ & 1.57860000 & -4.03555500 & 0.75679600 \\
\hline C & 1.03913700 & -4.79647700 & -1.74676300 \\
\hline C & 3.52227600 & -5.27864400 & -1.85837400 \\
\hline C & 4.65504100 & -2.30916300 & -2.29901200 \\
\hline C & 4.70748800 & -1.79746300 & 0.05313100 \\
\hline C & 0.49738100 & -4.18565600 & -2.87934200 \\
\hline C & 0.33885800 & -5.84712700 & -1.14530000 \\
\hline C & 3.32382500 & -5.92348500 & -3.08410400 \\
\hline C & 4.77909500 & -5.40717600 & -1.25406400 \\
\hline $\mathrm{H}$ & 4.14847200 & -2.76365000 & -3.14912200 \\
\hline C & 5.87298700 & -1.65970100 & -2.46154900 \\
\hline C & 5.92352600 & -1.13842100 & -0.10951500 \\
\hline
\end{tabular}


$\mathrm{H}$

$\mathrm{H}$

$\begin{array}{ccc}4.22412600 & -1.83600400 & 1.03091300 \\ 1.02715500 & -3.35656000 & -3.34354800 \\ -0.71550700 & -4.62184300 & -3.40532900 \\ -0.86158900 & -6.29823300 & -1.68172700 \\ 0.76306900 & -6.32980800 & -0.26462000 \\ 2.35656400 & -5.85325500 & -3.57589800 \\ 4.33983900 & -6.65993200 & -3.68382100 \\ 5.79480400 & -6.14251900 & -1.85129800 \\ 4.99060400 & -4.90860000 & -0.31027800 \\ 6.33004900 & -1.61165300 & -3.44772600 \\ 6.50848400 & -1.06879200 & -1.37034700 \\ 6.40197400 & -0.66686400 & 0.74912000 \\ -1.13462900 & -4.11697900 & -4.27262600 \\ -1.38572900 & -5.69015300 & -2.82052200 \\ -1.38842300 & -7.12647300 & -1.21207200 \\ 4.15219100 & -7.14895600 & -4.63724000 \\ 5.58197200 & -6.77441000 & -3.07182900 \\ 6.76082700 & -6.21848100 & -1.35679100 \\ -2.32493600 & -6.04277700 & -3.24389100 \\ 6.37702200 & -7.35027100 & -3.53974000 \\ 2.94710600 & 2.76447400 & -1.18877700 \\ 2.56136100 & 4.04443000 & -0.48028000 \\ 3.65291600 & 3.22063800 & 0.09832800 \\ 3.74872800 & 2.77774400 & -2.45121100 \\ 2.79890700 & 4.98928700 & -0.96067000 \\ 1.59198100 & 4.02151500 & 0.01886700 \\ 3.48496400 & 2.58649900 & 1.44448000 \\ 5.06822900 & 3.70824300 & -0.07797700 \\ 4.31374800 & 1.57354900 & -2.88572000 \\ 4.03416800 & 3.94210200 & -3.15884700 \\ 3.92692400 & 1.28582000 & 1.68373900 \\ 3.06881000 & 3.36794600 & 2.51984400 \\ 6.09044200 & 2.80980600 & -0.39530800 \\ 5.38785400 & 5.05241600 & 0.09935900 \\ 4.08915900 & 0.64727300 & -2.35040400 \\ 5.16496000 & 1.54392800 & -3.98001900 \\ 4.89027200 & 3.91513500 & -4.25663900 \\ 3.59432400 & 4.88807200 & -2.84676200 \\ 4.20385500 & 0.66200100 & 0.83620300 \\ 4.02451700 & 0.80028300 & 2.97739500 \\ 3.14860600 & 2.88307100 & 3.82219400 \\ 2.71507700 & 4.38184100 & 2.33115300 \\ 5.85203000 & 1.75639600 & -0.54205800 \\ 7.39394800 & 3.25378500 & -0.56824300\end{array}$




\begin{tabular}{|c|c|c|c|}
\hline C & 6.69661000 & 5.49936900 & -0.06150200 \\
\hline $\mathrm{H}$ & 4.59819100 & 5.75696100 & 0.35990200 \\
\hline $\mathrm{H}$ & 5.59906500 & 0.59653100 & -4.29418900 \\
\hline C & 5.46433400 & 2.71841100 & -4.66708900 \\
\hline $\mathrm{H}$ & 5.10900100 & 4.83745700 & -4.79057900 \\
\hline $\mathrm{H}$ & 4.41923300 & -0.20066200 & 3.14849200 \\
\hline C & 3.66028400 & 1.61037600 & 4.05550500 \\
\hline $\mathrm{H}$ & 2.85103400 & 3.51672800 & 4.65657800 \\
\hline $\mathrm{H}$ & 8.17228200 & 2.54295900 & -0.83984300 \\
\hline C & 7.70190000 & 4.60244200 & -0.40376400 \\
\hline $\mathrm{H}$ & 6.92869900 & 6.55351700 & 0.07529000 \\
\hline $\mathrm{H}$ & 3.81226200 & 1.26220700 & 5.07819100 \\
\hline $\mathrm{H}$ & 8.72259400 & 4.95173800 & -0.54320100 \\
\hline $\mathrm{H}$ & 7.45282200 & -0.54480600 & -1.50686100 \\
\hline $\mathrm{H}$ & 6.13775800 & 2.69769400 & -5.52114400 \\
\hline $\mathrm{H}$ & 1.40646800 & 7.29989600 & -1.70076400 \\
\hline $\mathrm{H}$ & -6.25975000 & -0.48436200 & -5.42251800 \\
\hline C & -0.45583800 & 0.10267600 & 2.11068800 \\
\hline C & -1.11321400 & 1.33231100 & 2.59924600 \\
\hline $\mathrm{O}$ & -0.25087300 & 2.36719400 & 2.57072000 \\
\hline C & -0.79932600 & 3.62810100 & 2.90683100 \\
\hline $\mathrm{H}$ & -0.03911300 & 4.37041100 & 2.64974500 \\
\hline $\mathrm{H}$ & -1.03664800 & 3.68683100 & 3.97725200 \\
\hline $\mathrm{H}$ & -1.72059900 & 3.81761300 & 2.34220300 \\
\hline $\mathrm{O}$ & -2.25849300 & 1.44436500 & 2.99763600 \\
\hline $\mathrm{O}$ & 0.66225000 & -2.65614900 & 4.18417200 \\
\hline $\mathrm{N}$ & 1.58819400 & -1.75497600 & 4.60680100 \\
\hline C & 2.95034900 & -2.13394200 & 4.45906800 \\
\hline C & 5.63099700 & -2.79007500 & 4.17689800 \\
\hline C & 3.33671900 & -3.00909800 & 3.44617000 \\
\hline C & 3.88342300 & -1.63990200 & 5.37206400 \\
\hline C & 4.68163500 & -3.32706000 & 3.31062300 \\
\hline C & 5.22650700 & -1.95523400 & 5.21455900 \\
\hline $\mathrm{H}$ & 2.59878900 & -3.42189200 & 2.76525000 \\
\hline $\mathrm{H}$ & 3.55519400 & -1.03169600 & 6.21205600 \\
\hline $\mathrm{H}$ & 4.99307000 & -3.99222100 & 2.50751900 \\
\hline $\mathrm{H}$ & 5.95511100 & -1.56086800 & 5.91760400 \\
\hline C & 0.15557500 & -2.25445200 & 2.80287400 \\
\hline $\mathrm{H}$ & 1.03852100 & -2.13592800 & 2.16499800 \\
\hline $\mathrm{H}$ & 6.68152400 & -3.03919800 & 4.05457800 \\
\hline C & -0.55299200 & -0.96593900 & 2.93395800 \\
\hline $\mathrm{H}$ & -1.26575800 & -0.92055100 & 3.75845400 \\
\hline C & -0.65787200 & -3.45214100 & 2.39425600 \\
\hline $\mathrm{H}$ & -1.53663900 & -3.57161700 & 3.03739300 \\
\hline
\end{tabular}




$\begin{array}{lccc}\mathrm{H} & -1.00284700 & -3.29119600 & 1.36608300 \\ \mathrm{H} & -0.05403200 & -4.36797900 & 2.43279400 \\ \mathrm{C} & -0.01054300 & -0.18755100 & 5.69603800 \\ \mathrm{C} & -0.92715200 & -1.10654700 & 6.23837700 \\ \mathrm{C} & -0.25401600 & 1.19100200 & 5.80437500 \\ \mathrm{C} & -2.06927200 & -0.63868300 & 6.86235400 \\ \mathrm{H} & -0.74816600 & -2.17322800 & 6.13046800 \\ \mathrm{C} & -1.41694600 & 1.64556500 & 6.40677300 \\ \mathrm{H} & 0.45676400 & 1.88863500 & 5.35862200 \\ \mathrm{C} & -2.32458000 & 0.73292800 & 6.93518500 \\ \mathrm{H} & -2.77888100 & -1.34552700 & 7.28565700 \\ \mathrm{H} & -1.62018800 & 2.71178100 & 6.46062400 \\ \mathrm{H} & -3.23803400 & 1.08792300 & 7.40531900 \\ \mathrm{C} & 1.21446900 & -0.56830200 & 5.04700800 \\ \mathrm{H} & 2.00739400 & 0.18108300 & 4.99468300\end{array}$

\begin{tabular}{|c|c|c|c|}
\hline \multicolumn{4}{|c|}{ r-TS(cx)-II (-5229.56637 a.u.) } \\
\hline $\mathrm{C}$ & 1.69818500 & -1.93671400 & -0.99733800 \\
\hline $\mathrm{C}$ & 1.92172600 & 1.73488500 & -1.10839200 \\
\hline $\mathrm{C}$ & -1.61845000 & 2.08415300 & -1.33275800 \\
\hline $\mathrm{C}$ & -2.11014100 & -1.58192500 & -1.21044400 \\
\hline $\mathrm{O}$ & 1.55141700 & 1.26808300 & 0.01406800 \\
\hline $\mathrm{O}$ & 1.44340200 & 1.45138200 & -2.24069900 \\
\hline 0 & -1.24516700 & 1.78813600 & -0.15229600 \\
\hline $\mathrm{O}$ & -1.42444800 & 1.40876200 & -2.37877700 \\
\hline $\mathrm{O}$ & -1.78515000 & -1.12781800 & -0.06988800 \\
\hline $\mathrm{O}$ & -1.45187100 & -1.46300400 & -2.27899600 \\
\hline 0 & 1.10075700 & -1.60939700 & 0.07507000 \\
\hline $\mathrm{O}$ & 1.55789000 & -1.41854300 & -2.13327900 \\
\hline $\mathrm{Rh}$ & -0.09472100 & 0.05972800 & 0.08506400 \\
\hline $\mathrm{Rh}$ & 0.03249300 & -0.03277600 & -2.36870300 \\
\hline $\mathrm{C}$ & -2.23348900 & 3.44597800 & -1.56299800 \\
\hline $\mathrm{C}$ & -3.26814300 & 3.54243000 & -2.63712800 \\
\hline $\mathrm{C}$ & -3.67709000 & 3.82115200 & -1.21705100 \\
\hline $\mathrm{C}$ & -1.15759900 & 4.49434100 & -1.51796900 \\
\hline $\mathrm{H}$ & -3.18757500 & 4.35576000 & -3.35370400 \\
\hline $\mathrm{H}$ & -3.60138000 & 2.59806300 & -3.06429100 \\
\hline $\mathrm{C}$ & -4.59907400 & 2.83479200 & -0.56580200 \\
\hline $\mathrm{C}$ & -3.93161500 & 5.24220000 & -0.77838700 \\
\hline $\mathrm{C}$ & -0.91933400 & 5.25600200 & -0.37230500 \\
\hline $\mathrm{C}$ & -0.38295800 & 4.72229700 & -2.65674600 \\
\hline $\mathrm{C}$ & -4.28456900 & 2.21161800 & 0.64223100 \\
\hline $\mathrm{C}$ & -5.87582600 & 2.65840400 & -1.10661600 \\
\hline $\mathrm{C}$ & -4.24823100 & 5.50739600 & 0.55946800 \\
\hline
\end{tabular}




\begin{tabular}{|c|c|c|c|}
\hline C & -3.80377700 & 6.32890600 & -1.64615800 \\
\hline $\mathrm{H}$ & -1.51709400 & 5.07384400 & 0.51823600 \\
\hline C & 0.02048800 & 6.27997800 & -0.38831800 \\
\hline C & 0.56976100 & 5.73635400 & -2.67039000 \\
\hline $\mathrm{H}$ & -0.54661000 & 4.10526100 & -3.53941600 \\
\hline $\mathrm{H}$ & -3.29121500 & 2.32277300 & 1.07090800 \\
\hline C & -5.24427000 & 1.46208100 & 1.31751400 \\
\hline C & -6.82594600 & 1.88674100 & -0.44852200 \\
\hline $\mathrm{H}$ & -6.12336200 & 3.15592200 & -2.04488700 \\
\hline $\mathrm{H}$ & -4.37552300 & 4.67790700 & 1.25313600 \\
\hline C & -4.39323400 & 6.81074100 & 1.01833100 \\
\hline C & -3.95120400 & 7.63426700 & -1.19048800 \\
\hline $\mathrm{H}$ & -3.56191100 & 6.16712900 & -2.69366200 \\
\hline $\mathrm{H}$ & 0.17149300 & 6.88767300 & 0.50190900 \\
\hline C & 0.75620200 & 6.53242800 & -1.54381400 \\
\hline $\mathrm{H}$ & 1.15941600 & 5.90965300 & -3.56828300 \\
\hline $\mathrm{H}$ & -4.98508800 & 1.00857300 & 2.27172200 \\
\hline C & -6.51689700 & 1.30246000 & 0.77835200 \\
\hline $\mathrm{H}$ & -7.81350400 & 1.75389600 & -0.88603800 \\
\hline $\mathrm{H}$ & -4.63119100 & 6.98696000 & 2.06536000 \\
\hline C & -4.23893900 & 7.88368400 & 0.14612900 \\
\hline $\mathrm{H}$ & -3.83662400 & 8.46045300 & -1.88877200 \\
\hline $\mathrm{H}$ & -7.26278100 & 0.71204100 & 1.30800300 \\
\hline $\mathrm{H}$ & -4.34980500 & 8.90478000 & 0.50392000 \\
\hline C & -3.40780500 & -2.33432500 & -1.37076300 \\
\hline C & -3.32975700 & -3.81009100 & -1.07892100 \\
\hline C & -4.24635600 & -2.96640900 & -0.25131500 \\
\hline C & -4.15605200 & -1.80646300 & -2.55883100 \\
\hline $\mathrm{H}$ & -3.74616900 & -4.50823100 & -1.80177000 \\
\hline $\mathrm{H}$ & -2.40458300 & -4.15258100 & -0.61349300 \\
\hline C & -4.03385200 & -2.83398400 & 1.23164100 \\
\hline C & -5.72175600 & -3.08083100 & -0.54808000 \\
\hline C & -4.57543500 & -0.47303700 & -2.52061100 \\
\hline C & -4.45623800 & -2.57595500 & -3.67682000 \\
\hline C & -3.86734000 & -1.61274100 & 1.88253100 \\
\hline C & -4.16772000 & -3.99350200 & 2.00310000 \\
\hline C & -6.53598000 & -1.95681700 & -0.39970500 \\
\hline C & -6.30881000 & -4.28795500 & -0.92104100 \\
\hline $\mathrm{H}$ & -4.34394700 & 0.14229000 & -1.64557600 \\
\hline C & -5.29807000 & 0.07073100 & -3.57273400 \\
\hline C & -5.18030700 & -2.03206300 & -4.73494900 \\
\hline $\mathrm{H}$ & -4.12046400 & -3.61117300 & -3.72387000 \\
\hline $\mathrm{H}$ & -3.73544200 & -0.70354900 & 1.30039900 \\
\hline C & -3.82530100 & -1.55421500 & 3.27438200 \\
\hline
\end{tabular}




\begin{tabular}{|c|c|c|c|}
\hline C & -4.12991300 & -3.93928700 & 3.39131400 \\
\hline $\mathrm{H}$ & -4.31187800 & -4.94830400 & 1.49658600 \\
\hline $\mathrm{H}$ & -6.08418400 & -1.01204700 & -0.10046500 \\
\hline C & -7.89873400 & -2.02900300 & -0.64881000 \\
\hline C & -7.67833700 & -4.36744700 & -1.16155600 \\
\hline $\mathrm{H}$ & -5.69048000 & -5.17895700 & -1.02467900 \\
\hline $\mathrm{H}$ & -5.63289400 & 1.10565500 & -3.51344700 \\
\hline C & -5.60643700 & -0.70994600 & -4.68480000 \\
\hline $\mathrm{H}$ & -5.41217500 & -2.64712900 & -5.60187200 \\
\hline $\mathrm{H}$ & -3.65805500 & -0.59129300 & 3.75864900 \\
\hline $\mathrm{C}$ & -3.95626700 & -2.71405100 & 4.03201100 \\
\hline $\mathrm{H}$ & -4.23580700 & -4.85289700 & 3.97280700 \\
\hline $\mathrm{H}$ & -8.51398800 & -1.13652900 & -0.54626900 \\
\hline $\mathrm{C}$ & -8.47651100 & -3.23722300 & -1.03295300 \\
\hline $\mathrm{H}$ & -8.12028000 & -5.31782200 & -1.45363200 \\
\hline $\mathrm{H}$ & -3.91960500 & -2.66107900 & 5.11903100 \\
\hline $\mathrm{H}$ & -9.54470000 & -3.29605900 & -1.22983400 \\
\hline C & 2.75132000 & -3.00670400 & -0.84982300 \\
\hline $\mathrm{C}$ & 2.52243500 & -4.08650300 & 0.15195300 \\
\hline C & 2.46233100 & -4.43748500 & -1.31315700 \\
\hline C & 4.10292400 & -2.37657400 & -0.97942000 \\
\hline $\mathrm{H}$ & 3.36744600 & -4.43402600 & 0.74252300 \\
\hline $\mathrm{H}$ & 1.56768800 & -4.07642100 & 0.67476400 \\
\hline C & 1.09591300 & -4.76909800 & -1.84644300 \\
\hline C & 3.58910800 & -5.22431600 & -1.92838300 \\
\hline C & 4.74413500 & -2.24832200 & -2.21285000 \\
\hline C & 4.69322700 & -1.82541800 & 0.15820700 \\
\hline C & 0.56192800 & -4.13077900 & -2.96764700 \\
\hline C & 0.38812200 & -5.83051600 & -1.27342600 \\
\hline C & 3.44018800 & -5.78036000 & -3.20351600 \\
\hline $\mathrm{C}$ & 4.81871200 & -5.39847300 & -1.28235900 \\
\hline $\mathrm{H}$ & 4.27526000 & -2.66905500 & -3.10127400 \\
\hline $\mathrm{C}$ & 5.97332400 & -1.60454400 & -2.29481200 \\
\hline $\mathrm{C}$ & 5.91844500 & -1.16932800 & 0.07512300 \\
\hline $\mathrm{H}$ & 4.17021300 & -1.90377500 & 1.11340700 \\
\hline $\mathrm{H}$ & 1.09749600 & -3.29395600 & -3.41100600 \\
\hline $\mathrm{C}$ & -0.65220100 & -4.54747500 & -3.50698700 \\
\hline $\mathrm{C}$ & -0.81465300 & -6.26041500 & -1.82156100 \\
\hline $\mathrm{H}$ & 0.80573300 & -6.33487000 & -0.40186700 \\
\hline $\mathrm{H}$ & 2.49338700 & -5.67199800 & -3.72730000 \\
\hline $\mathrm{C}$ & 4.48023200 & -6.47424400 & -3.81220000 \\
\hline $\mathrm{C}$ & 5.85862800 & -6.09171000 & -1.88870100 \\
\hline $\mathrm{H}$ & 4.98902200 & -4.97019200 & -0.29666600 \\
\hline $\mathrm{H}$ & 6.47745500 & -1.52651700 & -3.25580400 \\
\hline
\end{tabular}




\begin{tabular}{|c|c|c|c|}
\hline C & 6.56134500 & -1.05910100 & -1.15447200 \\
\hline $\mathrm{H}$ & 6.36001000 & -0.73466600 & 0.97205900 \\
\hline $\mathrm{H}$ & -1.06462800 & -4.02184500 & -4.36509000 \\
\hline C & -1.33299100 & -5.62207800 & -2.94635400 \\
\hline $\mathrm{H}$ & -1.34767800 & -7.09578800 & -1.37208300 \\
\hline $\mathrm{H}$ & 4.33283700 & -6.89393400 & -4.80491700 \\
\hline C & 5.69639400 & -6.63391100 & -3.15910100 \\
\hline $\mathrm{H}$ & 6.80336900 & -6.20589000 & -1.36160900 \\
\hline $\mathrm{H}$ & -2.27441600 & -5.95724400 & -3.37890500 \\
\hline $\mathrm{H}$ & 6.51059300 & -7.17639300 & -3.63394200 \\
\hline C & 3.00080300 & 2.77396800 & -1.10452900 \\
\hline C & 2.60364800 & 4.05009600 & -0.39499900 \\
\hline C & 3.68180900 & 3.22088200 & 0.20033800 \\
\hline C & 3.82690000 & 2.79544100 & -2.35098700 \\
\hline $\mathrm{H}$ & 2.85002900 & 4.99689500 & -0.86680500 \\
\hline $\mathrm{H}$ & 1.62663100 & 4.02527200 & 0.09006500 \\
\hline C & 3.46758400 & 2.57933400 & 1.53581000 \\
\hline C & 5.10189100 & 3.70198200 & 0.05727000 \\
\hline C & 4.39801900 & 1.59282700 & -2.78111500 \\
\hline C & 4.12490700 & 3.96304900 & -3.04779300 \\
\hline C & 3.85231500 & 1.26097500 & 1.77269000 \\
\hline C & 3.02679500 & 3.35952800 & 2.60195500 \\
\hline C & 6.12707100 & 2.79651900 & -0.22842300 \\
\hline C & 5.42304900 & 5.04564500 & 0.23550000 \\
\hline $\mathrm{H}$ & 4.16459500 & 0.66496800 & -2.25223600 \\
\hline C & 5.26525000 & 1.56659700 & -3.86274500 \\
\hline C & 4.99823800 & 3.93975700 & -4.13204400 \\
\hline $\mathrm{H}$ & 3.68156600 & 4.90817900 & -2.73815800 \\
\hline $\mathrm{H}$ & 4.15892200 & 0.64094400 & 0.93219400 \\
\hline C & 3.83819600 & 0.74385300 & 3.05748000 \\
\hline C & 2.99703300 & 2.84335100 & 3.89421600 \\
\hline $\mathrm{H}$ & 2.72551100 & 4.39062800 & 2.41369500 \\
\hline $\mathrm{H}$ & 5.88620500 & 1.74336700 & -0.37402000 \\
\hline C & 7.43695200 & 3.23324900 & -0.36876600 \\
\hline C & 6.73782700 & 5.48520400 & 0.10710600 \\
\hline $\mathrm{H}$ & 4.63049200 & 5.75543300 & 0.47185300 \\
\hline $\mathrm{H}$ & 5.70266500 & 0.62009900 & -4.17486300 \\
\hline C & 5.57629000 & 2.74394500 & -4.53964000 \\
\hline $\mathrm{H}$ & 5.22700500 & 4.86422200 & -4.65796500 \\
\hline $\mathrm{H}$ & 4.17217300 & -0.27819700 & 3.22879700 \\
\hline C & 3.42666000 & 1.54152500 & 4.12746300 \\
\hline $\mathrm{H}$ & 2.66376500 & 3.46782700 & 4.72183500 \\
\hline $\mathrm{H}$ & 8.21927000 & 2.51755400 & -0.61460500 \\
\hline C & 7.74716600 & 4.58127600 & -0.20325600 \\
\hline
\end{tabular}




\begin{tabular}{|c|c|c|c|}
\hline $\mathrm{H}$ & 6.97171400 & 6.53883000 & 0.24471500 \\
\hline $\mathrm{H}$ & 3.46376600 & 1.15266400 & 5.14520700 \\
\hline $\mathrm{H}$ & 8.77307500 & 4.92457800 & -0.31673100 \\
\hline $\mathrm{H}$ & 7.51666700 & -0.54253700 & -1.22761100 \\
\hline $\mathrm{H}$ & 6.26254400 & 2.72636300 & -5.38344900 \\
\hline $\mathrm{H}$ & 1.48487200 & 7.34105600 & -1.56166700 \\
\hline $\mathrm{H}$ & -6.17756600 & -0.28777600 & -5.50863100 \\
\hline C & -0.40714800 & 0.01405200 & 2.08652000 \\
\hline C & -1.08112500 & 1.23087000 & 2.58849100 \\
\hline $\mathrm{O}$ & -0.22403800 & 2.26133900 & 2.63875300 \\
\hline C & -0.79891900 & 3.52179000 & 2.94411500 \\
\hline $\mathrm{H}$ & -0.01779600 & 4.26304600 & 2.75779900 \\
\hline $\mathrm{H}$ & -1.12453900 & 3.56629000 & 3.99127000 \\
\hline $\mathrm{H}$ & -1.66618700 & 3.71358400 & 2.30115900 \\
\hline $\mathrm{O}$ & -2.25425000 & 1.31705400 & 2.89850600 \\
\hline $\mathrm{O}$ & 0.50023900 & -2.90823100 & 4.05849200 \\
\hline$N$ & 1.35047900 & -1.97924300 & 4.59633100 \\
\hline C & 2.73135300 & -2.25222200 & 4.49464100 \\
\hline C & 5.47061500 & -2.70628100 & 4.34257500 \\
\hline C & 3.22938800 & -3.14648700 & 3.54580700 \\
\hline C & 3.59019600 & -1.64379000 & 5.41300400 \\
\hline C & 4.59965300 & -3.36071000 & 3.47399700 \\
\hline C & 4.95912200 & -1.85744900 & 5.32023400 \\
\hline H & 2.55092700 & -3.65994300 & 2.87105800 \\
\hline $\mathrm{H}$ & 3.17711000 & -1.02145400 & 6.20424100 \\
\hline $\mathrm{H}$ & 4.99315900 & -4.03973400 & 2.71962000 \\
\hline $\mathrm{H}$ & 5.62595700 & -1.36870200 & 6.02581200 \\
\hline C & 0.12962800 & -2.41432600 & 2.69416400 \\
\hline $\mathrm{H}$ & 1.05776100 & -2.29726300 & 2.12282100 \\
\hline $\mathrm{H}$ & 6.54163300 & -2.87503300 & 4.27037400 \\
\hline C & -0.52104700 & -1.09666800 & 2.88038800 \\
\hline $\mathrm{H}$ & -1.35015500 & -1.10427800 & 3.59026900 \\
\hline C & -0.71884300 & -3.52306500 & 2.13035100 \\
\hline H & -1.64726100 & -3.63660800 & 2.69946000 \\
\hline $\mathrm{H}$ & -0.96960300 & -3.27697600 & 1.09257400 \\
\hline $\mathrm{H}$ & -0.16905600 & -4.47263000 & 2.15335200 \\
\hline C & -0.32467800 & -0.43700700 & 5.62029800 \\
\hline C & -1.14850900 & -1.40935100 & 6.20941200 \\
\hline C & -0.62014500 & 0.92270100 & 5.79325100 \\
\hline C & -2.24801400 & -1.01333000 & 6.95229900 \\
\hline $\mathrm{H}$ & -0.93205100 & -2.46336000 & 6.05111300 \\
\hline C & -1.73582700 & 1.30781400 & 6.52265500 \\
\hline H & 0.02369000 & 1.66656200 & 5.31997100 \\
\hline C & -2.55155700 & 0.34148700 & 7.10243500 \\
\hline
\end{tabular}




$\begin{array}{crrc}\mathrm{H} & -2.88319900 & -1.76544800 & 7.41492600 \\ \mathrm{H} & -1.97122000 & 2.36298800 & 6.63644500 \\ \mathrm{H} & -3.42703000 & 0.64048600 & 7.67327500 \\ \mathrm{C} & 0.84579900 & -0.75322200 & 4.82801100 \\ \mathrm{H} & 1.56938200 & 0.05444400 & 4.68118600\end{array}$

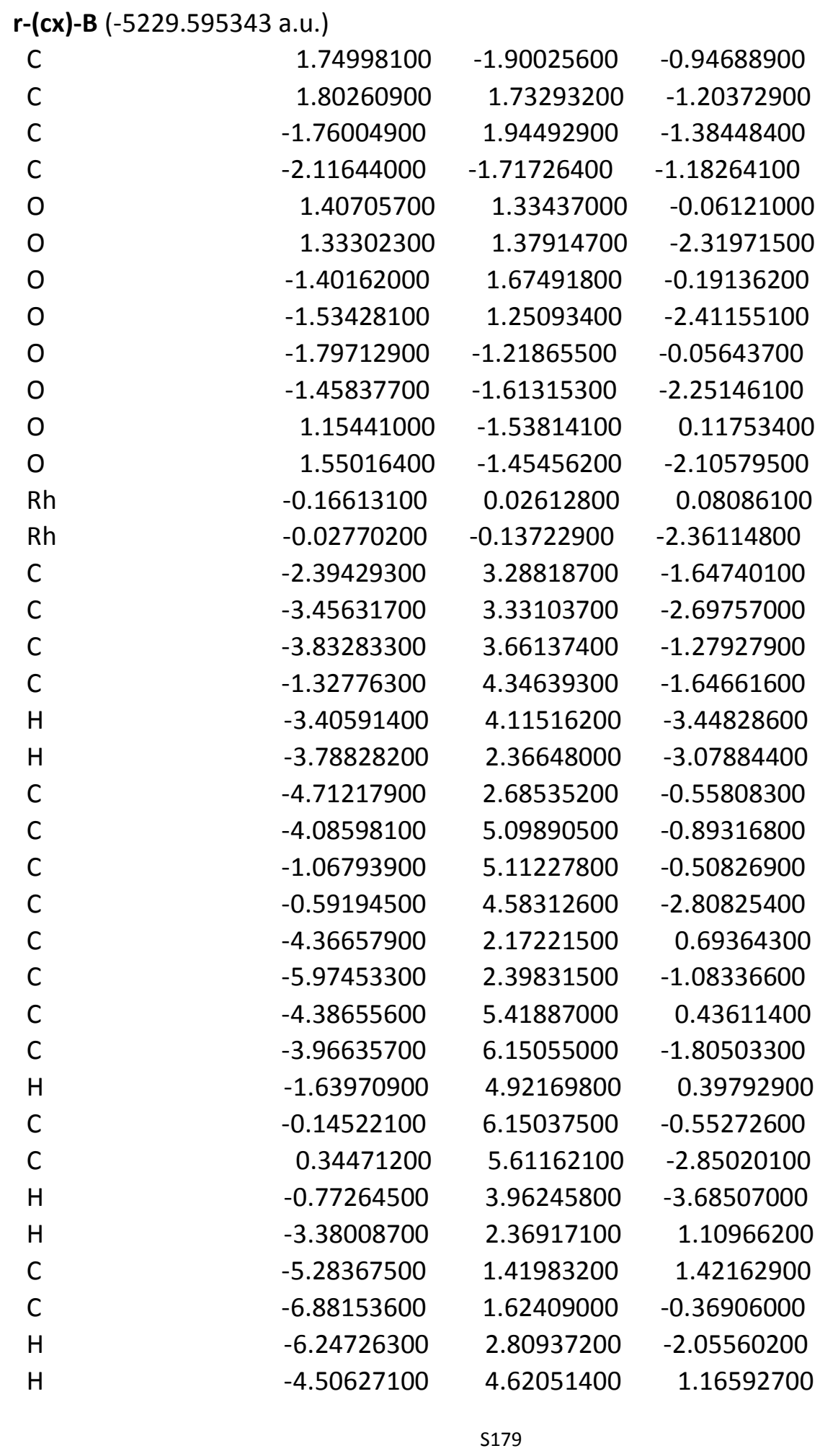




\begin{tabular}{|c|c|c|c|}
\hline C & -4.52480400 & 6.73989600 & 0.84403300 \\
\hline C & -4.10619800 & 7.47321100 & -1.40028900 \\
\hline $\mathrm{H}$ & -3.73722100 & 5.94900200 & -2.84842100 \\
\hline $\mathrm{H}$ & 0.02241200 & 6.76270700 & 0.33130900 \\
\hline C & 0.55278600 & 6.41090500 & -1.72993000 \\
\hline $\mathrm{H}$ & 0.90308500 & 5.79472200 & -3.76583000 \\
\hline $\mathrm{H}$ & -5.00546100 & 1.04710300 & 2.40515500 \\
\hline C & -6.54295500 & 1.14776100 & 0.89581400 \\
\hline $\mathrm{H}$ & -7.85807500 & 1.40270500 & -0.79539700 \\
\hline $\mathrm{H}$ & -4.75067600 & 6.95762900 & 1.88580000 \\
\hline C & -4.37860100 & 7.77666800 & -0.07152500 \\
\hline $\mathrm{H}$ & -3.99821200 & 8.27030200 & -2.13254500 \\
\hline $\mathrm{H}$ & -7.25384500 & 0.55190200 & 1.46578900 \\
\hline $\mathrm{H}$ & -4.48360700 & 8.81142600 & 0.24625500 \\
\hline C & -3.39686800 & -2.49747500 & -1.30023300 \\
\hline C & -3.29258900 & -3.95528100 & -0.92968900 \\
\hline C & -4.20949000 & -3.08107100 & -0.13721100 \\
\hline C & -4.15900500 & -2.04134200 & -2.50804200 \\
\hline $\mathrm{H}$ & -3.70827900 & -4.69136000 & -1.61423700 \\
\hline $\mathrm{H}$ & -2.35901900 & -4.26190900 & -0.45554000 \\
\hline C & -3.95847700 & -2.86064900 & 1.32814600 \\
\hline C & -5.68850000 & -3.22813000 & -0.39537100 \\
\hline C & -4.62469900 & -0.72291000 & -2.51911800 \\
\hline C & -4.41349400 & -2.85829200 & -3.60349200 \\
\hline C & -3.83585900 & -1.59836900 & 1.90753700 \\
\hline C & -3.99484700 & -3.98265900 & 2.16114100 \\
\hline C & -6.51725000 & -2.11315000 & -0.25965600 \\
\hline C & -6.26450700 & -4.45608100 & -0.71318700 \\
\hline $\mathrm{H}$ & -4.42385200 & -0.07145300 & -1.66249700 \\
\hline C & -5.34873300 & -0.23932900 & -3.59907900 \\
\hline C & -5.13787400 & -2.37404800 & -4.69010600 \\
\hline $\mathrm{H}$ & -4.03737900 & -3.88093800 & -3.61179500 \\
\hline $\mathrm{H}$ & -3.78709100 & -0.71465900 & 1.27453100 \\
\hline C & -3.73674100 & -1.46401500 & 3.29065200 \\
\hline C & -3.89713800 & -3.85372200 & 3.54177500 \\
\hline $\mathrm{H}$ & -4.10313000 & -4.96942600 & 1.71022400 \\
\hline $\mathrm{H}$ & -6.07508400 & -1.15227700 & -0.00036500 \\
\hline C & -7.88548400 & -2.21608900 & -0.46351200 \\
\hline C & -7.63896600 & -4.56534900 & -0.91059900 \\
\hline $\mathrm{H}$ & -5.63519400 & -5.34069000 & -0.80297200 \\
\hline $\mathrm{H}$ & -5.72067800 & 0.78431100 & -3.58095200 \\
\hline C & -5.61015200 & -1.06666600 & -4.68952400 \\
\hline $\mathrm{H}$ & -5.33300100 & -3.02358100 & -5.54061100 \\
\hline $\mathrm{H}$ & -3.60863700 & -0.47149600 & 3.72370800 \\
\hline
\end{tabular}




\begin{tabular}{|c|c|c|c|}
\hline C & -3.76640500 & -2.58969600 & 4.11063400 \\
\hline $\mathrm{H}$ & -3.92107700 & -4.73907500 & 4.17297000 \\
\hline $\mathrm{H}$ & -8.51388000 & -1.33209600 & -0.36691100 \\
\hline C & -8.45285400 & -3.44534800 & -0.79224600 \\
\hline $\mathrm{H}$ & -8.07267600 & -5.53175600 & -1.15865000 \\
\hline $\mathrm{H}$ & -3.69332400 & -2.48022500 & 5.19155000 \\
\hline $\mathrm{H}$ & -9.52537500 & -3.52869000 & -0.95287100 \\
\hline C & 2.85353400 & -2.90806300 & -0.76085000 \\
\hline C & 2.73236300 & -3.93807200 & 0.31201700 \\
\hline C & 2.63254000 & -4.38151600 & -1.12306400 \\
\hline C & 4.18593300 & -2.25645400 & -0.97131100 \\
\hline $\mathrm{H}$ & 3.62190100 & -4.16506800 & 0.89533600 \\
\hline $\mathrm{H}$ & 1.80368400 & -3.96572900 & 0.87578200 \\
\hline C & 1.26954300 & -4.84892700 & -1.55710300 \\
\hline C & 3.77739300 & -5.10806300 & -1.77510200 \\
\hline C & 4.74877500 & -2.13446200 & -2.24353500 \\
\hline C & 4.85684300 & -1.72168400 & 0.12943400 \\
\hline C & 0.58120100 & -4.29196300 & -2.63689500 \\
\hline C & 0.71758300 & -5.95754100 & -0.90687400 \\
\hline C & 3.67678300 & -5.48468200 & -3.11875000 \\
\hline C & 4.97405800 & -5.37632700 & -1.10627200 \\
\hline $\mathrm{H}$ & 4.21872100 & -2.54731900 & -3.10034400 \\
\hline C & 5.98194700 & -1.51403800 & -2.40242700 \\
\hline C & 6.07801700 & -1.07277600 & -0.03410400 \\
\hline $\mathrm{H}$ & 4.41034600 & -1.81979000 & 1.12197600 \\
\hline $\mathrm{H}$ & 0.99246300 & -3.42233800 & -3.14490400 \\
\hline C & -0.63023600 & -4.83569300 & -3.05908500 \\
\hline C & -0.48267300 & -6.51036100 & -1.33510400 \\
\hline $\mathrm{H}$ & 1.25463000 & -6.39599400 & -0.06574700 \\
\hline $\mathrm{H}$ & 2.74935300 & -5.29910200 & -3.65704400 \\
\hline C & 4.74221500 & -6.08743900 & -3.77601100 \\
\hline C & 6.04120300 & -5.98036700 & -1.76143300 \\
\hline $\mathrm{H}$ & 5.09204000 & -5.10106400 & -0.06072800 \\
\hline $\mathrm{H}$ & 6.43025000 & -1.44916500 & -3.39164100 \\
\hline C & 6.64471900 & -0.97457400 & -1.30140200 \\
\hline $\mathrm{H}$ & 6.57975000 & -0.64009100 & 0.83045500 \\
\hline $\mathrm{H}$ & -1.16303200 & -4.37148200 & -3.88669400 \\
\hline C & -1.15502300 & -5.95364900 & -2.42140800 \\
\hline $\mathrm{H}$ & -0.89161200 & -7.37978600 & -0.82496000 \\
\hline $\mathrm{H}$ & 4.63846000 & -6.36524100 & -4.82262100 \\
\hline C & 5.93290700 & -6.33551800 & -3.10099600 \\
\hline $\mathrm{H}$ & 6.96302400 & -6.17344700 & -1.21722300 \\
\hline $\mathrm{H}$ & -2.09440000 & -6.38584300 & -2.76243600 \\
\hline $\mathrm{H}$ & 6.76843500 & -6.80606700 & -3.6139980 \\
\hline & & S181 & \\
\hline
\end{tabular}




\begin{tabular}{|c|c|c|c|}
\hline C & 2.89740400 & 2.75269500 & -1.25294500 \\
\hline C & 2.56463500 & 4.05725400 & -0.56103600 \\
\hline C & 3.64152400 & 3.21164100 & 0.01326800 \\
\hline C & 3.66654300 & 2.73525200 & -2.53716800 \\
\hline $\mathrm{H}$ & 2.81942100 & 4.98080900 & -1.07361100 \\
\hline $\mathrm{H}$ & 1.60887500 & 4.07877400 & -0.03680200 \\
\hline C & 3.45483300 & 2.62177000 & 1.37790300 \\
\hline C & 5.06910200 & 3.64470700 & -0.19131100 \\
\hline C & 4.25986600 & 1.53203500 & -2.93216300 \\
\hline C & 3.85945700 & 3.86645400 & -3.32402500 \\
\hline C & 3.69275200 & 1.27448600 & 1.64952600 \\
\hline C & 3.17610700 & 3.48921200 & 2.43204300 \\
\hline C & 6.06104400 & 2.68590100 & -0.40988700 \\
\hline C & 5.43618100 & 4.98614600 & -0.11385200 \\
\hline $\mathrm{H}$ & 4.11161600 & 0.63318100 & -2.32753600 \\
\hline C & 5.03425100 & 1.46747100 & -4.08019100 \\
\hline C & 4.64071600 & 3.80541100 & -4.47544800 \\
\hline $\mathrm{H}$ & 3.39908100 & 4.81097900 & -3.03872200 \\
\hline $\mathrm{H}$ & 3.88609000 & 0.58898200 & 0.82475100 \\
\hline C & 3.67274400 & 0.80840600 & 2.95682500 \\
\hline C & 3.15154500 & 3.02759900 & 3.74402000 \\
\hline $\mathrm{H}$ & 2.99883900 & 4.54352200 & 2.21512700 \\
\hline $\mathrm{H}$ & 5.78248200 & 1.63421100 & -0.47285200 \\
\hline C & 7.38660300 & 3.06348100 & -0.57034300 \\
\hline C & 6.76634300 & 5.36767100 & -0.26777300 \\
\hline $\mathrm{H}$ & 4.67156300 & 5.74035000 & 0.07049300 \\
\hline $\mathrm{H}$ & 5.48712200 & 0.52066400 & -4.36774900 \\
\hline C & 5.23227200 & 2.60772200 & -4.85616900 \\
\hline $\mathrm{H}$ & 4.78547200 & 4.70127700 & -5.07540600 \\
\hline $\mathrm{H}$ & 3.86425800 & -0.24463800 & 3.16690800 \\
\hline C & 3.41429100 & 1.68883100 & 4.00843600 \\
\hline $\mathrm{H}$ & 2.94364400 & 3.71680300 & 4.56131100 \\
\hline $\mathrm{H}$ & 8.14445600 & 2.30388300 & -0.75365500 \\
\hline C & 7.74448700 & 4.40801900 & -0.50098400 \\
\hline $\mathrm{H}$ & 7.03727600 & 6.41955100 & -0.20643100 \\
\hline $\mathrm{H}$ & 3.41993600 & 1.32259200 & 5.03324900 \\
\hline $\mathrm{H}$ & 8.78299700 & 4.70528000 & -0.62817700 \\
\hline $\mathrm{H}$ & 7.60272600 & -0.47576300 & -1.43420300 \\
\hline $\mathrm{H}$ & 5.84423000 & 2.55978300 & -5.75411400 \\
\hline $\mathrm{H}$ & 1.26768200 & 7.23077600 & -1.77038100 \\
\hline $\mathrm{H}$ & -6.18108500 & -0.69176200 & -5.53592700 \\
\hline C & -0.31965500 & 0.05845200 & 2.03188300 \\
\hline C & -0.89090500 & 1.32874800 & 2.53388400 \\
\hline $\mathrm{O}$ & -0.01263400 & 2.32172700 & 2.52794500 \\
\hline
\end{tabular}




\begin{tabular}{|c|c|c|c|}
\hline C & -0.53106300 & 3.61014100 & 2.85009400 \\
\hline $\mathrm{H}$ & 0.22712800 & 4.32503500 & 2.52351500 \\
\hline $\mathrm{H}$ & -0.69858700 & 3.68857100 & 3.93080300 \\
\hline $\mathrm{H}$ & -1.47745900 & 3.78147500 & 2.32613100 \\
\hline $\mathrm{O}$ & -2.06893300 & 1.41153100 & 2.81773300 \\
\hline 0 & 0.45908400 & -2.95404000 & 4.01374300 \\
\hline $\mathrm{N}$ & 1.07124900 & -1.95757200 & 4.78939200 \\
\hline C & 2.47617800 & -2.10903300 & 4.83626000 \\
\hline C & 5.26583400 & -2.28386200 & 4.98736000 \\
\hline C & 3.15685200 & -3.09700000 & 4.11865600 \\
\hline C & 3.19414900 & -1.25026800 & 5.67463600 \\
\hline C & 4.54399700 & -3.16992900 & 4.19278500 \\
\hline C & 4.57886300 & -1.33276600 & 5.73813500 \\
\hline $\mathrm{H}$ & 2.59358400 & -3.80279200 & 3.51469300 \\
\hline $\mathrm{H}$ & 2.64783700 & -0.52716000 & 6.27950400 \\
\hline $\mathrm{H}$ & 5.06472900 & -3.93358900 & 3.61728600 \\
\hline $\mathrm{H}$ & 5.12460700 & -0.64868900 & 6.38486400 \\
\hline C & 0.34192500 & -2.40814400 & 2.69343300 \\
\hline $\mathrm{H}$ & 1.34499100 & -2.29207000 & 2.25383500 \\
\hline $\mathrm{H}$ & 6.34979500 & -2.34539500 & 5.03642900 \\
\hline C & -0.23101200 & -1.03057200 & 3.01480400 \\
\hline $\mathrm{H}$ & -1.29591700 & -1.17337300 & 3.30691000 \\
\hline C & -0.50611700 & -3.38003600 & 1.92019000 \\
\hline $\mathrm{H}$ & -1.49978400 & -3.46132600 & 2.37206800 \\
\hline $\mathrm{H}$ & -0.60951500 & -3.07316900 & 0.87689200 \\
\hline $\mathrm{H}$ & -0.04019500 & -4.37306300 & 1.95287100 \\
\hline C & -0.26999100 & 0.07703000 & 5.32776900 \\
\hline C & -1.34190500 & -0.57210700 & 5.94273000 \\
\hline C & -0.01166500 & 1.41234200 & 5.62847400 \\
\hline C & -2.16981800 & 0.11899200 & 6.81543900 \\
\hline $\mathrm{H}$ & -1.51807100 & -1.62459600 & 5.71947800 \\
\hline C & -0.84125100 & 2.10548100 & 6.50833000 \\
\hline $\mathrm{H}$ & 0.83675100 & 1.91108900 & 5.15564900 \\
\hline C & -1.92573100 & 1.46379400 & 7.09379100 \\
\hline $\mathrm{H}$ & -3.01001500 & -0.39042200 & 7.28339400 \\
\hline $\mathrm{H}$ & -0.63539100 & 3.14945000 & 6.73743200 \\
\hline $\mathrm{H}$ & -2.57799300 & 2.00581800 & 7.77454100 \\
\hline C & 0.56470200 & -0.64725400 & 4.31207600 \\
\hline $\mathrm{H}$ & 1.42119100 & -0.01839600 & 4.02630900 \\
\hline
\end{tabular}

